\title{
Molecular characterization of sub-cuticular bacteria in New Zealand echinoderms
}

\author{
A thesis \\ submitted in partial fulfillment \\ of the requirements \\ for the degree \\ of
}

Master of Science in Marine Biology

At

Victoria University of Wellington

By

\section{Scott Anthony Lawrence}

\author{
School of Biological Sciences \\ Victoria University of Wellington \\ New Zealand \\ 2008
}




\section{Abstract}

Many echinoderms contain sub-cuticular bacteria (SCB), symbionts which reside in the lumen between the epidermal cells and the outer cuticle of the host. The relationship is very common, with $\sim 60 \%$ of all echinoderms studied so far containing SCB. Currently, little is known about the function of the symbiosis, although it has been hypothesized that SCB may aid in host nutrition or antimicrobial defense. Whatever their function, the large numbers of SCB observed in many echinoderms $\left(10^{8}-10^{9} \mathrm{SCB} \mathrm{g}^{-1}\right.$ AFDW host tissue) suggest that they may be important to the host. Factors contributing to the lack of knowledge about the echinoderm-SCB symbiosis include the difficulty associated with cultivating symbiotic bacteria, and the lack of studies identifying the SCB by molecular means.

In this study, molecular techniques were employed to characterize the SCB of several common New Zealand echinoderms. The specific objectives of the study were to identify the SCB through sequencing of a region of the bacterial 16S rRNA gene, identify and locate SCB in situ through the use of fluorescence in situ hybridization (FISH), and cultivate SCB obtained from those echinoderms which were found to contain them.

Phylogenetic analysis of 16S rRNA sequences obtained from echinoderm-associated bacteria resulted in the identification of four putative species of SCB. All four bacteria were isolated from samples of Stichopus mollis (class Holothuroidea), and two of the four were also found in samples of Patiriella sp (class Asteroidea). The first putative SCB belongs to the order Rhizobiales ( $\alpha$-proteobacteria), and is closely related to the SCB 
previously isolated from the brittle star Ophiactis balli. The second species belongs to the order Chromatiales ( $\gamma$-proteobacteria). Putative SCB species 3 falls within the Roseobacter clade ( $\alpha$-proteobacteria). The phylogenetic placement of the final putative SCB is more ambiguous, as this bacterium falls among members of the $\alpha$ - and $\gamma$ subdivisions of the phylum Proteobacteria. The nearest relatives of this final bacterium are in the orders Rickettsiales and Thiotrichales.

Results of FISH assays show that Patiriella sp. and S. mollis contain SCB, while a third species, Astrostole scabra (class Asteroidea) does not. The SCB community composition was found to vary between Patiriella sp. and S. mollis. In both species, the majority of the SCB present were found to belong to the $\alpha$-subdivision of the phylum Proteobacteria ( $>80 \%$ in both species). However, in S. mollis, $\sim 20 \%$ of the SCB community consists of bacteria belonging to the $\gamma$-subdivision of the phylum Proteobacteria, whereas bacteria belonging to this subdivision were never observed in Patiriella sp.

Cultivation experiments were carried out using a range of culture media, however results were inconclusive. Ten species of proteobacteria were successfully cultivated, three of which were obtained only from Patiriella sp. and S. mollis samples and were considered possible candidates for SCB. However, phylogenetic analysis of these three bacteria revealed that closely-related bacteria are predominantly free-living species. While the possibility remains that these three bacteria are in fact SCB, it seems more likely that they represent seawater or echinoderm surface-associated bacteria. 
This study contributes to the body of knowledge of the echinoderm-SCB symbiosis by identifying several potential SCB in Patiriella sp. and S. mollis, and is the first to identify SCB in situ through the use of FISH. An obvious goal in studies of the echinoderm-SCB symbiosis is to determine the function of the relationship. Potential functions of the symbiosis, based on the results obtained here, are discussed herein. 


\section{Acknowledgments}

I would like to express thanks first and foremost to my supervisors, Dr Simon Davy and Dr Ronan O'Toole. I am particularly thankful to Simon for suggesting this research topic and for his willingness to help whenever needed.

Sincere thanks must also be given to Dr Mike Taylor at the University of Auckland, whose assistance with the phylogenetic software package ARB was invaluable. Thanks also go to Mike for running CARD-FISH assays on my samples.

To Simon Thomson and Dr Matthew Chan, thank you for your help during my early days in the lab; without your help I would have been truly lost. The past and present students of the KK816 and KK817 labs also deserve thanks for offering advice and taking the time to help out when things stopped working.

Numerous technical staff provided vital assistance throughout this project. Dr Jo Davy provided the FISH protocol used in this study, as well as general advice on FISH. Sushila Pilai and Dan Logan taught me how to use the confocal laser scanning microscope. Alan Hoverd provided a great deal of useful advice on histology techniques.

I am indebted to Nathan Smith and Jason Temel, who were always willing to help with sample collection, often in less than ideal weather conditions. Thank you also to Kate Neill at NIWA, who provided the deep-water echinoderms used in this study. 
Thanks must go to the excellent administrative staff in the School of Biological Sciences: Mary Murray, Sandra Taylor, Patricia Stein, Paul Marsden and Delwyn Carter-Jarratt.

Special thanks go to Maria Hook, who often took time out of her busy schedule to read earlier versions of this manuscript, and who was a great source of support throughout the last couple of years.

This research was made possible by funding from Victoria University of Wellington, in the form of a Victoria Master's (by thesis) Scholarship and a Faculty of Science Small Research Grant to S. Lawrence, and a University Research Fund Grant to S. Davy. 


\section{Table of contents}

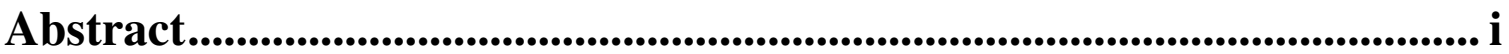

Acknowledgments ....................................................................................................... iv

List of figures.............................................................................................................. ix

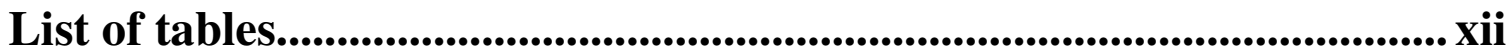

Chapter 1: General introduction............................................................ 1

1.1 Symbiosis ........................................................................................................................ 1

1.2 Marine invertebrate - microbe symbiosis .................................................................... 3

1.3 Echinoderm - sub-cuticular bacteria symbiosis .................................................... 13

Phylum Echinodermata ...................................................................................... 13

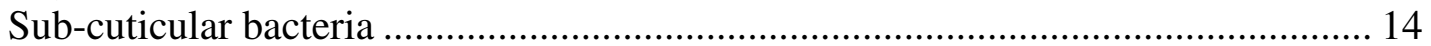

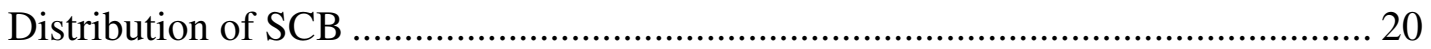

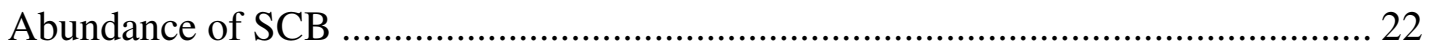

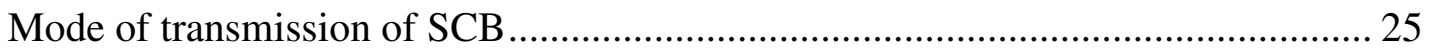

Functional roles of the echinoderm-SCB symbiosis............................................... 26

1.4. Aim and specific objectives ........................................................................................ 31

Chapter 2: Phylogenetic identification of sub-cuticular bacteria ........... 33

Abstract.......................................................................................................................... 33

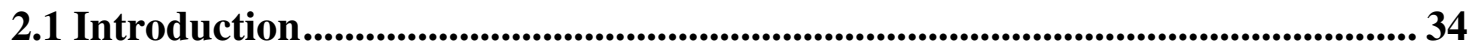

2.2 Materials and methods ...................................................................................................... 37

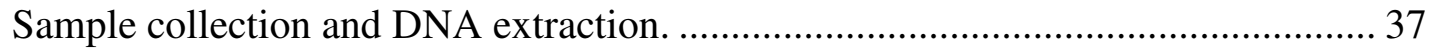

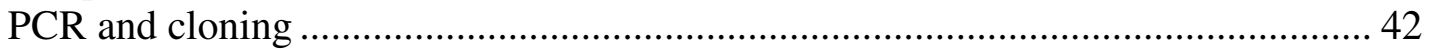

Sequencing and phylogenetic analysis.................................................................... 43

2.3 Results .............................................................................................................................. 45

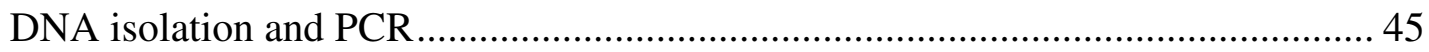

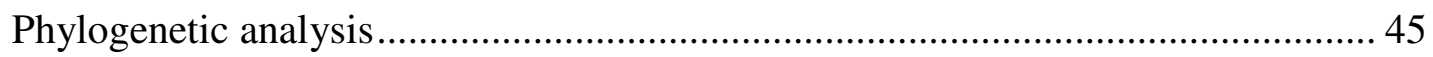

2.4 Discussion....................................................................................................................................... 58

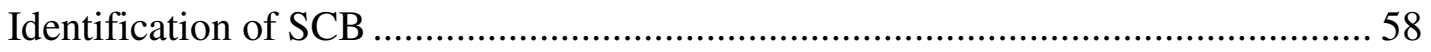




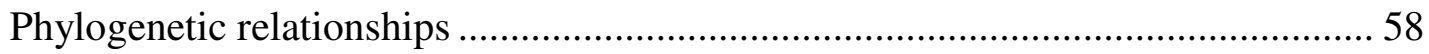

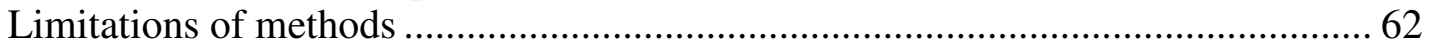

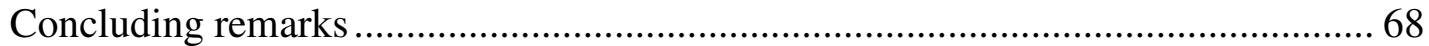

\section{Chapter 3: Fluorescence in situ hybridization analysis of sub-cuticular}

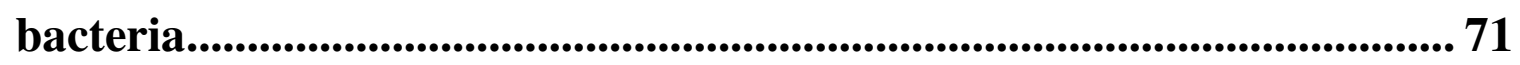

Abstract.................................................................................................................................... 71

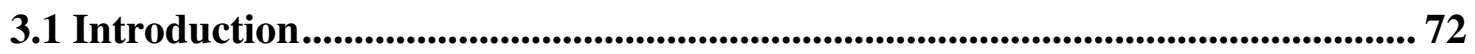

3.2 Materials and methods ................................................................................................. 75

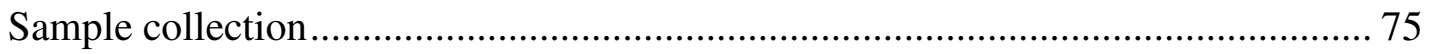

Sample fixation and decalcification....................................................................... 76

Tissue processing, embedding and sectioning ........................................................ 76

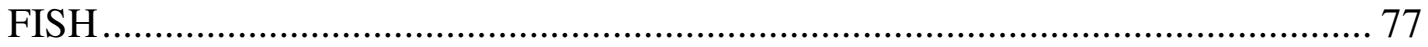

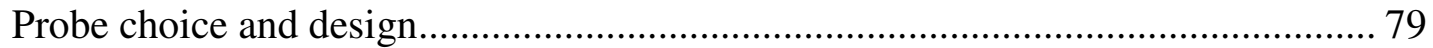

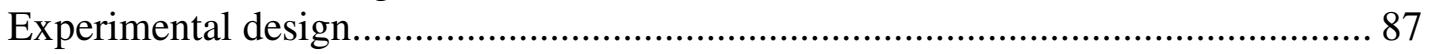

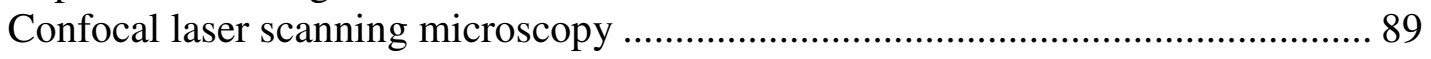

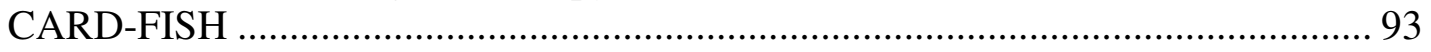

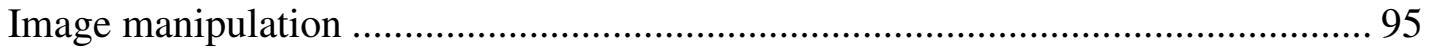

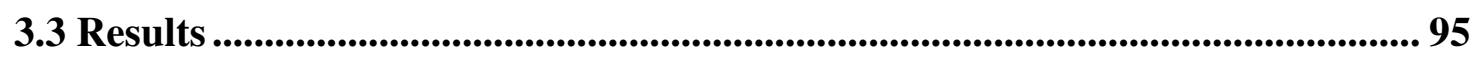

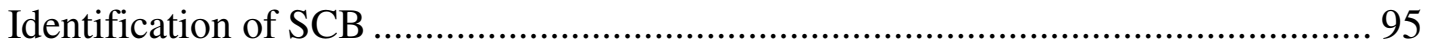

SCB community composition ........................................................................ 100

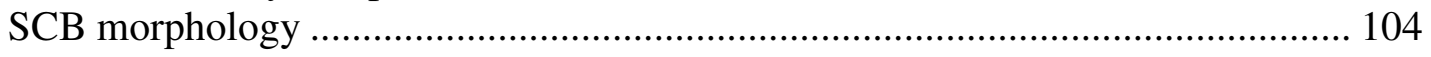

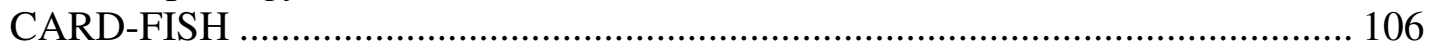

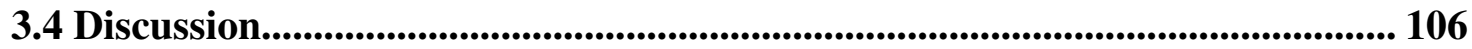

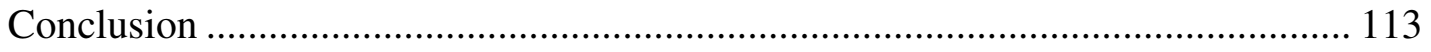

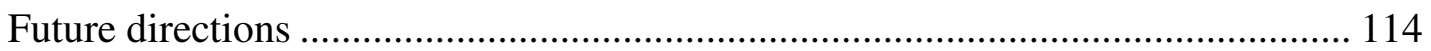

Chapter 4: Cultivation of sub-cuticular bacteria ..................................... 116

Abstract......................................................................................................................... 116

4.1 Introduction .................................................................................................................... 117

4.2 Materials and methods ............................................................................................ 119

Bacterial isolation and growth conditions.......................................................... 119 
Culture media.

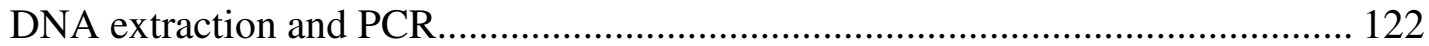

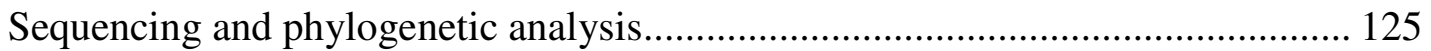

4.3 Results ................................................................................................................. 126

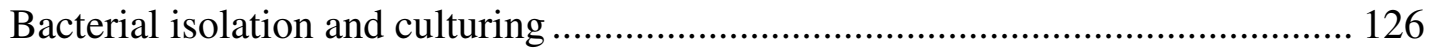

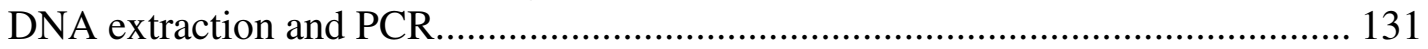

Sequencing and phylogenetic analysis......................................................... 132

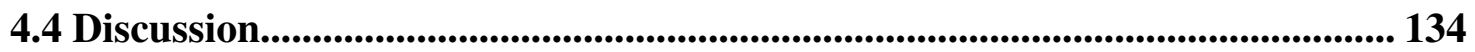

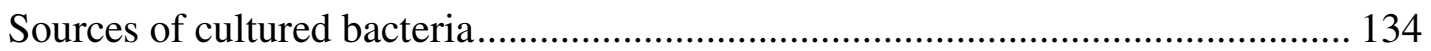

Reasons for inconclusive results from cultivation ............................................ 138

Concluding remarks ......................................................................... 144

Chapter 5: General discussion..................................................................... 146

5.1 Summary of results ................................................................................................................. 146

5.2 Potential roles of SCB in the symbiosis................................................................... 149

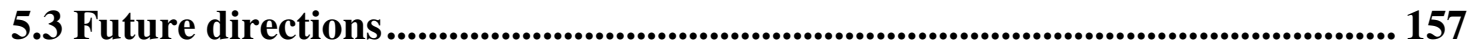

References......................................................................................................... 161

Appendix ............................................................................................................................. 191

Chapter 2 (Phylogenetic analysis of SCB) ................................................................ 191

Development of methods ................................................................................. 191

Phylogenetic trees of bacteria identified from seawater and echinoderm surfaces 198

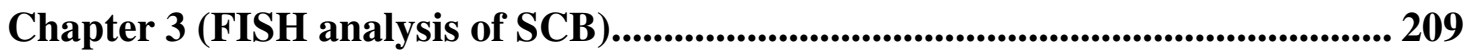

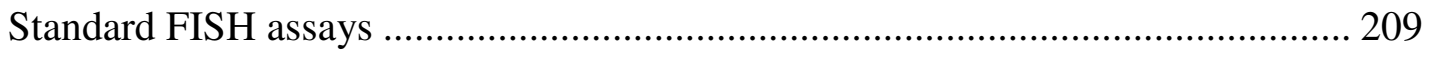

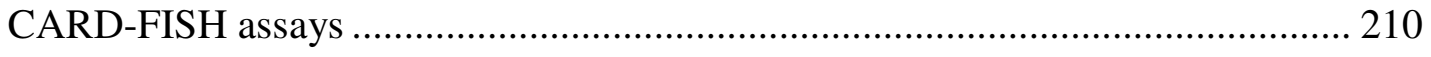

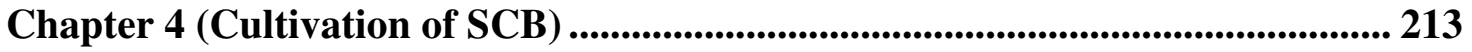




\section{List of figures}

Figure 1.1. Transmission electron micrograph of the asteroid Odontaster benhami tube foot.

Figure 1.2. Transmission electron micrograph of the bursal area of the ophiuroid Amphipholis squamata. 15

Figure 1.3. Transmission electron micrograph showing the Type 1 SCB (arrows) present in Ophiothrix fragilis.... .18

Figure 1.4. Transmission electron micrograph showing the Type 2 SCB present in Astrobrachion constrictum. .18

Figure 1.5. Transmission electron micrograph of the Type 3 (Subtype 3) SCB present in Pseudechinus huttoni. 19

Figure 2.1. Ventral (oral) and dorsal (aboral) views of Patiriella sp...................39

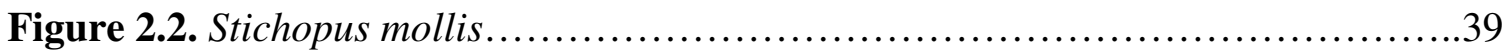

Figure 2.3. Astrostole scabra. A: Dorsal (aboral) view. B: Ventral (oral) view...........40

Figure 2.4. Map showing location of shallow-water echinoderm collection .............40

Figure 2.5. 16S rRNA-based phylogeny of putative SCB species $1 \ldots \ldots \ldots \ldots \ldots \ldots . . .54$

Figure 2.6. $16 \mathrm{~S}$ rRNA-based phylogeny of putative SCB species $2 \ldots \ldots \ldots \ldots \ldots \ldots \ldots 5$

Figure 2.7. 16S rRNA-based phylogeny of putative SCB species $3 \ldots \ldots \ldots \ldots \ldots \ldots \ldots . \ldots 6$

Figure 2.8. 16S rRNA-based phylogeny of putative SCB species $4 \ldots \ldots \ldots \ldots \ldots \ldots . . \ldots 7$

Figure 3.1. Secondary structure of the $16 \mathrm{~S}$ rRNA of Escherichia coli, showing the

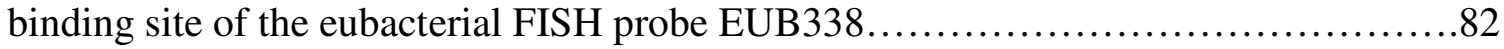

Figure 3.2. Secondary structure of the $16 \mathrm{~S}$ rRNA of the $\alpha$-proteobacterium Roseovarius tolerans, showing the binding site of the alpha-proteobacteria specific FISH probe ALF968.

Figure 3.3. Secondary structure of part of the $16 \mathrm{~S}$ rRNA of SCB species 1, showing the binding site of the species-specific FISH probe SCB1.... .84

Figure 3.4. Secondary structure of part of the $16 \mathrm{~S}$ rRNA of SCB species 2, showing the binding site of the species-specific FISH probe SCB2. .85 
Figure 3.5. Secondary structure of the $23 \mathrm{~S}$ rRNA of Escherichia coli showing the binding site of the $\gamma$-proteobacteria-specific FISH probe GAM42a..................86

Figure 3.6. Excitation and emission spectra for the dye Cy3 ..................... 90

Figure 3.7. Excitation and emission spectra for the dye Cy5 .................... 90

Figure 3.8. Excitation and emission spectra for the DNA stain DAPI...............91

Figure 3.9. Excitation and emission spectra for the dye FITC .....................91

Figure 3.10. Unlabeled echinoderm sections, showing the autofluorescence of the

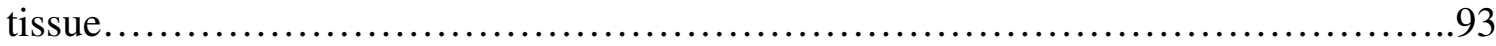

Figure 3.11. CLSM images of EUB338 hybridizations.............................97

Figure 3.12. CLSM images of ALF968 hybridizations.......................... 99

Figure 3.13. CLSM image of GAM42a hybridization in Stichopus mollis.............100

Figure 3.14. CLSM images of bacteria hybridized with EUB338, ALF968 and GAM42a probes.

Figure 3.15. CLSM images showing longitudinal view of SCB hybridized with the eubacterial probe EUB338. .105

Figure 3.16. CLSM images showing longitudinal view of SCB from Stichopus mollis .105

Figure 3.17. Diagram of $16 \mathrm{~S}$ rRNA, showing relative accessibility of different regions 110

Figure 4.1. Diagram outlining the sampling and culturing process

Figure 4.1. Petri dishes containing Marine Agar 2216, after 48 hours at $4{ }^{\circ} \mathrm{C}$. 128

Figure 4.2. Petri dishes containing Marine Agar $2216+$ Patiriella sp. homogenate after 48 hours at $4{ }^{\circ} \mathrm{C}$. 128

Figure 4.3. Petri dishes containing Marine Agar $2216+$ S. mollis homogenate after 48 hours at $4{ }^{\circ} \mathrm{C}$....

Figure 4.4. Petri dishes containing Agar + Patiriella sp. homogenate after 48 hours at 4 ${ }^{\circ} \mathrm{C}$. 129

Figure 4.5. Petri dishes containing Agar $+S$. mollis homogenate after 48 hours at 4 ${ }^{\circ} \mathrm{C}$.

Figure 4.6. Petri dishes containing Zobell modified 2216E medium after 48 hours at 4 ${ }^{\circ} \mathrm{C}$. 
Figure 4.7. 16S rRNA-based phylogeny of cultured bacteria from echinoderm and seawater samples.

Figure 4.8. Diagram outlining SCB isolation methods used by Strahl et al (2002).....140

Figure A1. 16S rRNA-based phylogeny of A. scabra surface-associated $\alpha$ proteobacteria.

Figure A2. 16S rRNA-based phylogeny of A. scabra surface-associated $\gamma$ - and $\beta$ proteobacteria .200

Figure A3. 16S rRNA-based phylogeny of Patiriella sp. -associated $\alpha$ - and $\varepsilon$ proteobacteria. .201

Figure A4. 16S rRNA-based phylogeny of Patiriella sp--associated $\gamma$ - and $\beta$ proteobacteria. .202

Figure A5. 16S rRNA-based phylogeny of $S$. mollis.-associated $\alpha^{-}$and $\varepsilon^{-}$ proteobacteria.

Figure A6. 16S rRNA-based phylogeny of $S$. mollis.-associated $\gamma$ - and $\beta$ proteobacteria. .204

Figure A7. 16S rRNA-based phylogeny of Perissasterias monocantha-, Psilaster acuminatus- and Rosaster mimicus-associated $\alpha$-proteobacteria..... .205

Figure A8. 16S rRNA-based phylogeny of Psilaster acuminatus- and Rosaster mimicusassociated $\gamma$ - and $\beta$-proteobacteria. .206

Figure A9. 16S rRNA-based phylogeny of $\alpha$ - and $\delta$-proteobacteria isolated from seawater. 207

Figure A10. 16S rRNA-based phylogeny of $\gamma$-proteobacteria isolated from

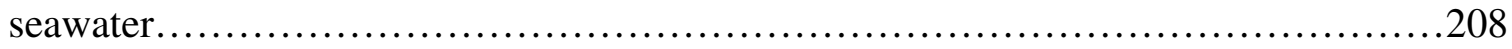

Figure A11. Result of CARD-FISH assay using the FITC-labeled eubacterial probe EUB338 on Patiriella sp. tissue sections

Figure A12. Result of CARD-FISH assay using the FITC-labeled eubacterial probe EUB338 on $S$. mollis tissue sections 


\section{List of tables}

Table 1.1. SCB loads of symbiotic echinoderms...............................24

Table 2.1. Relationship of SCB species 1 to published sequences....................48

Table 2.2. Relationship of SCB species 2 to published sequences....................49

Table 2.3. Relationship of SCB species 3 to published sequences...................50

Table 2.4. Relationship of SCB species 4 to published sequences....................51

Table 3.1. FISH probes used in this study................................... 80

Table 3.2. FISH probe combinations used to determine SCB community composition..88

Table 3.3. Results of FISH assays, carried out using each probe individually..........96

Table 3.4. Proportion of SCB communities of Patiriella sp. and Stichopus mollis made

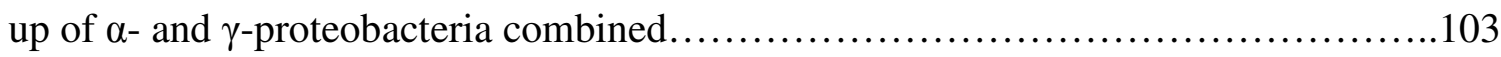

Table 3.5. Proportion of SCB communities of Patiriella sp. and Stichopus mollis made up of $\alpha$ - and $\gamma$-proteobacteria. .104

Table 4.1. Results of culturing assays...................................... 127

Table 4.2. Suggested culture media for the growth of SCB identified in three echinoderm species.

Table A1. PCR primers trialed in this study, and the reasons for discontinuation of their

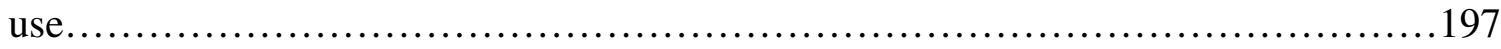

Table A2. Formula for FISH hybridization buffer...............................209

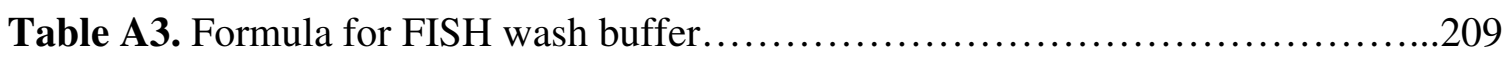

Table A4. Specificity of oligonucleotide probes SCB1 and SCB2 $\ldots \ldots \ldots \ldots \ldots \ldots \ldots . \ldots \ldots$

Table A5. Formula for CARD-FISH hybridization buffer........................210

Table A6. Formula for CARD-FISH wash buffer..............................211

Table A7. Formula for CARD-FISH amplification buffer.......................211

Table A8. Approximate formula of Difco Marine Broth 2216......................213

Table A9. Formula of Zobell modified 2216E medium..........................214

Table A10. Formula of Artificial Seawater used in this study......................214 


\section{Chapter 1: General introduction}

\subsection{Symbiosis}

The term 'symbiosis' ("symbiotismus") was coined by the lichenologist A.B. Frank in 1877 to describe a close relationship between different species. This original definition, which included only mutually beneficial interactions, was expanded upon by H.A de Bary in 1879 to include parasitic relationships. De Bary described three criteria necessary for a relationship to be considered symbiotic: 1) two organisms must be living together; 2) the two organisms must be in physical contact with each other; and 3) the two organisms must be different species. Despite the common misconception that symbiosis is synonymous with mutualism, the correct usage of the term follows de Bary's broad definition of symbiosis, covering the range of interactions from parasitism to mutualism. Thus there are three potential relationships which are considered symbioses: mutualism, commensalism and parasitism. Mutualism describes a relationship in which both partners benefit from their association with each other; commensalism is a relationship which is beneficial to one partner, and neither beneficial nor detrimental to the other partner; parasitism occurs when one partner benefits at the expense of the other partner. In reality, a continuum exists between mutualism and parasitism, and relationships which are mutualistic can often become parasitic when the appropriate conditions arise. For example, a microbial mutualist of a plant may benefit the host by suppressing pathogens or resisting disease, and in turn gain energy from the host plant. In the absence of pathogens, however, the plant derives no benefit from the association, and the relationship effectively becomes parasitic (Preston 2004). In addition to the range of 
relationships above, symbioses can be further divided into obligate (in which at least one partner is dependent on the relationship for survival) and facultative (in which the relationship is not necessary for survival of either partner) symbioses; and endo- (one partner living inside the other) and ectosymbioses (one partner living on the other).

Symbiosis has played an important role in the evolution of eukaryotes. It has been hypothesized that the eukaryotic nucleus arose from a symbiotic virus (Bell 2001, Takemura 2001), while the symbiotic origin of mitochondria and chloroplasts (Margulis 1981, Gray 1989) is now widely accepted. In the cases of mitochondria and chloroplasts, structural and molecular analyses have shown that their closest relatives are $\alpha$ proteobacteria and cyanobacteria, respectively (McFadden 1999, Kutschera \& Niklas 2005). It has been suggested that "symbiogenesis" (a symbiotic association which causes evolution) could be responsible for many instances of rapid evolutionary change and speciation events, given the length of time required for such events to take place by natural selection alone (Roossinck 2005). It is evident that the course of evolution has been significantly influenced by symbiosis, but of greater relevance to the current study is the contemporary importance of symbiotic relationships.

Symbiosis is widespread, with practically all animals containing prokaryotic endosymbionts (Phillips 2006) and over $80 \%$ of land plants forming symbioses with mycorrhizal fungi (Newman \& Reddell 1987). These are two very general examples, and in truth, organisms of every shape and size form symbioses with a diverse array of other organisms. Furthermore, one species will often form symbioses with several other species. 
In such cases, the symbionts (the smaller partner in the relationship) can range from closely-related, species with similar functions (such as the several species of predatory mites which are provided with shelter by the tropical tree Cupania vernalis and prey upon herbivores in return (Romero \& Benson 2004)), to unrelated species playing very different roles, such as the parasitic nematodes (Williamson \& Gleason 2003) and mutualistic mycorrhizal fungi (Kogel et al. 2006) which both infect plant roots.

\subsection{Marine invertebrate - microbe symbiosis}

In her review of symbioses between invertebrates and microorganisms, Saffo (1992) describes several general features of such relationships, illustrating their ecological and evolutionary importance. First, symbiosis is widespread among invertebrates: there is no species in which at least some individuals are not hosts of another species. Second, symbiotic invertebrates often carry a large symbiont load; for example, microorganisms can make up more than half of a sponge's mass (Brantley et al. 1995). Third, endosymbiosis has been important in shaping the evolution of some invertebrates. This can take place through co-evolution of host and symbiont, or by alteration of host ecology or metabolism by symbionts. Fourth, invertebrate endosymbiosis can have an ecological impact, by altering interactions between the host species and others in the ecosystem, or by allowing the host to utilize different resources or expand its range. A well-known example is that of scleractinian corals, which often contain photosynthetic symbionts that allow the corals to live in nutrient-poor tropical waters (Stanley 2006). Reefs formed by symbiotic corals in turn provide a rich habitat for numerous other species in an otherwise inhospitable part of the ocean. 
Despite the abundance and importance of invertebrate-microbe symbioses, the vast majority of information comes from terrestrial systems. Marine invertebrate symbioses have been largely overlooked in comparison to their terrestrial counterparts, with a few notable exceptions such as sponge-microbe associations and the aforementioned coralalga mutualism. Likely explanations for this bias towards terrestrial species include the relative inaccessibility of marine ecosystems, and the fact that many of the terrestrial species which have received attention are of economic importance (for example, those involving agriculturally important plants). Despite this bias, certain marine invertebrate taxa have received considerable attention, particularly in recent years with the advent of molecular approaches such as PCR and DNA sequencing. Molecular approaches have, in many cases, reduced the reliance on cultivation of symbiotic microbes, thus eliminating a major hurdle in understanding marine microbial interactions. Furthermore, the relatively new field of metagenomics allows the reconstruction of near-complete genomes of the various microbes present in environmental samples, allowing a vast range of analyses to be carried out on microbial symbionts without the need for isolation or cultivation (Allen \& Banfield 2005).

A better understanding of marine invertebrate-microbe symbioses is desirable for several reasons. First, a better understanding of such symbioses would enhance our knowledge of the general biology of many invertebrates. For example, in gutless tube worms (phylum Annelida), the discovery of chemosynthetic bacterial symbionts (Cavanaugh et al. 1981) revealed how the hosts are able to survive with no conventional means of feeding. Given 
the diversity of invertebrates present in the marine environment, and the prevalence of microbial symbionts (Saffo 1992), more research into these relationships is likely to yield fascinating insights into the physiologies and lifestyles of the invertebrate hosts.

Second, more information about marine invertebrate-microbe symbioses is needed in order to better conserve marine biodiversity. Symbioses between marine invertebrates can have significant effects on ecosystem functioning. Examples include reef-building corals and their algal symbionts (Stanley 2006), and the chemosynthetic bacteria found in invertebrates at hydrothermal vents, which form the basis of the food web (Cavanaugh et al. 1981, Distel et al. 1995). As noted by Smith (2001), one of the best ways to minimize loss of biodiversity is to preserve whole ecosystems, and a thorough understanding of symbioses such as those just mentioned is vital to understanding how the organisms in an ecosystem interact.

Third, more research into marine invertebrate-microbe symbioses is desirable for biotechnological reasons. Marine invertebrates are a rich source of bioactive secondary metabolites, many of which are drug candidates. Recently it has been hypothesized that many of these bioactive compounds are in fact produced by microbial symbionts of these invertebrates, rather than by the invertebrates themselves (Piel 2004, König et al. 2006, Piel 2006). Currently, one of the major difficulties in successful drug development from invertebrate-derived secondary metabolites is the production of sufficient quantities of the compound of interest. For example, to isolate $18 \mathrm{~g}$ of the anti-cancer drug bryostatin from the bryozoan Bugula neritina, $13 \mathrm{t}$ of the animal were required (Schaufelberger et al. 
1991). If drug candidates are in fact produced by symbiotic microbes, the isolation and cultivation of these microbes may provide an alternative method for isolating compounds of interest. Although the majority of bacteria are not cultivable by conventional means (Hugenholtz 2002), identification of symbiotic microbes by molecular means may help in the selection of appropriate media and growth conditions. In situations where the host invertebrate is rare and/or not amenable to aquaculture, this approach may be the only way to obtain sufficient quantities of secondary metabolites.

In order to provide the reader with a feeling for the range of marine invertebrate-microbe symbioses that exist, examples from several of the better studied marine invertebrate taxa are provided below.

\section{Corals (phylum Cnidaria)}

Of all the invertebrate-microbe symbioses present in the marine environment, the relationship between reef-building corals and their photosynthetic microalgal symbionts (dinoflagellates of the genus Symbiodinium) is perhaps the most well studied, and certainly one of the more charismatic, given the aesthetic and commercial values of coral reefs. The photosynthetic symbionts of corals are found within host endodermal cells, and provide the host with energy-rich photosynthate, allowing them to persist in unproductive tropical waters (Stanley \& Swart 1995, Stanley 2006). In return, the symbionts gain a permanent position in the water column and are provided with nitrogen, phosphorous and carbon dioxide by the host. Recently, this symbiosis has received considerable attention due to an increase in bleaching events (stress-induced loss of algal symbionts) (Wilkinson 
1999, Douglas 2003). A major driver of bleaching is an increase in temperature of the surrounding seawater, and coral reefs are expected to fare badly in response to predicted increases in global temperature over the next century (Hoegh-Guldberg 1999).

In addition to the well known coral-dinoflagellate association, corals form symbioses with bacteria. There appear to be two distinct bacterial communities associated with corals, one of which is associated with coral tissues (as either endo- or ectosymbionts), and the other of which is associated with the mucus layer covering coral tissues (Bourne \& Munn 2005, Koren \& Rosenberg 2006). The identities of coral-associated bacteria have only been established for a few coral species, but it appears that coral-associated bacterial assemblages are made up predominantly of proteobacteria and cyanobacteria (Rohwer et al. 2001, Bourne \& Munn 2005, Koren \& Rosenberg 2006). Little is known of the role the bacteria may be playing in the symbiosis, but $20 \%$ of bacteria cultured from the mucus of Acropora palmata displayed antibiotic activity, and mucus samples from this coral were found to inhibit a potentially infectious bacterium (Ritchie 2006). In addition to this apparent antimicrobial function, bacteria are thought to aid in uptake of nutrients by the coral, and at least one coral has been shown to contain nitrogen-fixing bacteria (Lesser et al. 2004).

Corals have also been shown to contain virus-like particles (VLPs) in their mucus layer which resemble a range of previously described viruses. The diversity of VLPs suggests that there is a range of hosts present in the coral mucus, including bacteria, algae, fungi, and the coral itself (Davy \& Patten 2007). Very few studies of coral-associated viruses 
have been carried out, and as yet there is no indication of what their symbiotic function may be.

\section{Sponges (phylum Porifera)}

The symbiosis between sponges and microbes (in particular bacteria) has been extensively studied, with various workers focusing on the identity of associated microbes, their mode of transmission, the function of the symbiosis, and the potential for isolating bioactive compounds from the sponge or symbionts. An in-depth analysis of the state of knowledge of sponge-microbe symbioses is beyond the scope of the present study; rather, a brief overview is provided of the symbiosis between sponges and bacteria. The interested reader is directed to the excellent review of sponge-associated microorganisms by Taylor et al. (2007).

Sponges are efficient filter feeders, capable of filtering thousands of litres of seawater each day (Reiswig 1974). A large proportion of the nutrients obtained by sponges from seawater comes in the form of microorganisms such as bacteria and microalgae (Reiswig 1975, Wehrl et al. 2007). Most of these microorganisms are phagocytosed, but in many sponge species a significant number of bacteria (up to $10^{8}-10^{10}$ bacteria per gram wet sponge weight) remain intact and form symbioses with the sponge (Vacelet \& Donadey 1977, Hentschel et al. 2006). It has been shown that sponges can distinguish between symbiotic bacteria and food, avoiding phagocytosis of symbionts (Wilkinson et al. 1984, Wehrl et al. 2007). Sponge symbionts include representatives from at least 13 existing bacterial phyla, as well as the sponge-specific candidate phylum "Poribacteria" (Fieseler 
et al. 2004, Taylor et al. 2007). Symbiotic bacteria may provide the sponge with nutrition via translocation of dissolved organic matter or photosynthate (cyanobacterial symbionts) (Wilkinson 1983, Webster \& Hill 2001), they may aid in defense against pathogenic microbes (Hentschel et al. 2001, Lee \& Qian 2004), or they may metabolize host waste products (Wilkinson 1978). One reason sponge-microbe symbioses are of particular interest is that sponges are one of the major sources of novel bioactive compounds, and more sponge-derived compounds are in drug trials than compounds from any other marine organism (Blunt et al. 2005, 2006, Taylor et al. 2007). As with other marine invertebrates, the bioactive compounds isolated from sponges are often thought to come from their bacterial partners, so the interest in this symbiosis is likely to continue for some time.

\section{Squid and bivalves (phylum Mollusca)}

Some of the most interesting symbioses in the marine environment are those between squid and luminous bacteria. The best studied of these is the relationship between the bobtail squid Euprymna scolopes and the proteobacterium Vibrio fischeri. V. fischeri is a luminous bacterium that infects the light organ of E. scolopes, allowing the squid to emit light and camouflage itself from predators (Ruby 1996, Mcfall-Ngai 1999). This is a highly specific relationship, involving a complex physiological response on the part of the host: upon initiation of the symbiosis, the host's light organ changes from a morphology suited to accepting symbionts to one more suited to utilizing the bacterial luminescence (Ruby 1996, Mcfall-Ngai 1999). Another interesting feature of this symbiosis is that, despite the specificity of the relationship, each generation of the squid 
acquires the bacteria from seawater, rather than transmission from parent to offspring (Nyholm et al. 2000, Visick \& Ruby 2006).

Bivalve molluscs are another group in which microbial symbionts are common and reasonably well studied. Bivalve-associated bacteria generally belong to the proteobacteria, and are most often associated with the gills of the host (Distel et al. 1988, Krueger \& Cavanaugh 1997, Elsaied et al. 2006, Spiridonova et al. 2006, Duperron et al. 2007, Scott \& Cavanaugh 2007). The symbiosis appears to be quite specific, with bivalves often containing only one or two species of bacteria (Distel et al. 1988, Krueger \& Cavanaugh 1997). Functions of the microbial symbionts are varied, but most commonly involve a nutritional or antimicrobial role. Good examples of the proposed antimicrobial role of symbionts come from the scallop Argopecten purpuratus, from which symbiotic bacteria with antimicrobial action against pathogenic Vibrio spp. have been isolated (Riquelme et al. 1996, Jorquera et al. 1999). The predominant mode of nutrition among bivalve symbionts is chemoautotrophy, particularly in sulphide rich environments such as hydrothermal vents, where sulphur-oxidizing symbionts are the host's main source of energy (Distel et al. 1988, Elsaied et al. 2006). Such symbioses are not restricted to hydrothermal vents, however; similar chemoautotrophic bacteria are found in bivalves from sulphide-rich coastal environments (Durand et al. 1996, Frenkiel et al. 1996), and methanotrophic bacteria are found in bivalves from methane-rich environments such as cold seeps (Elsaied et al. 2006, Spiridonova et al. 2006). 
Bryozoans (phylum Bryozoa)

Although not as extensively studied as some other marine invertebrate taxa, certain bryozoan species have received considerable attention, due in part to the finding of a group of anticancer drugs known as bryostatins in the bryozoan Bugula neritina (Pettit et al. 1982). Recently it has been suggested, based on similarity of bryostatins to bacterial secondary metabolites, and the inability of B. neritina to produce bryostatins when bacterial symbionts are removed, that these compounds are in fact manufactured by the bryozoan's endosymbiotic bacteria (Davidson et al. 2001). Bryostatins are thought to play a role in anti-predator defense of the bryozoan, as several have been found to be unpalatable to predators such as fish (Lopanik et al. 2004). Other bryozoan species are also known to contain similar proteobacterial symbionts (Anderson \& Haygood 2007), but as yet their functions and biotechnological potentials are unknown.

\section{Polychaetes and oligochaetes (phylum Annelida)}

Annelids, in particular polychaetes, are very common in the marine environment, and the phylum is one of the most species-rich in the oceans (Costello et al. 2006). The worms in this phylum are very diverse in terms of morphology, physiology and habitat, ranging from gutless, heat-tolerant, hydrothermal vent-associated tubeworms (Desbruyères et al. 1998, Chao et al. 2007) to free-living predatory worms of shallow waters (Arndt \& Schiedek 1997). Likewise, the symbioses between annelids and microorganisms are varied. The best known annelid-microbe symbioses are those involving gutless tube worms living near hydrothermal vents (e.g. Alvinella pompejana and Ridgeia piscesae), in which nutrition is provided entirely by chemoautotrophic, sulphur-oxidizing symbiotic 
bacteria (Desbruyères et al. 1998). This chemoautotrophic association is not restricted to tube worms, as sulphur-oxidizing and sulphate-reducing chemoautotrophic bacteria are also found in free-living oligochaete worms living in oxygen deficient sediments, such as Olavius algarvensis (Woyke et al. 2006) and O. crassitunicatus (Blazejak et al. 2005). One of the more unusual annelid-microbe symbioses is that between polychaetes of the genus Osedax and their bacterial partners. Osedax spp. feed on whale carcasses, by boring through bones to feed on the lipids inside; however these worms lack a mouth and gut, and rely on proteobacterial symbionts living intracellularly in root-like projections to digest the lipids (Goffredi et al. 2005). As can be seen in the examples above, symbioses between annelids and microorganisms are interesting from an evolutionary point of view because of the high degree of specialization and co-evolution often involved.

There are over 20 invertebrate phyla present in the marine environment, consisting of thousands of species, most of which are probably symbiotic with microorganisms. Some of these symbioses are well known, though many are likely as yet undescribed or poorly understood. One group of marine invertebrates that is known to form a range of symbioses with microorganisms is the phylum Echinodermata. The best studied of these interactions is that between echinoderms and sub-cuticular bacteria, which is described in the following section. 


\subsection{Echinoderm - sub-cuticular bacteria symbiosis}

\section{Phylum Echinodermata}

The phylum Echinodermata is a large group of marine invertebrates, consisting of approximately 7,000 species. This phylum is divided into five classes: Asteroidea (sea stars), Crinoidea (sea lilies or feather stars), Echinoidea (sea urchins), Holothuroidea (sea cucumbers) and Ophiuroidea (brittle stars). Echinoderms are geographically widespread and occur at all depths, from the intertidal zone to the deep sea, where they are often one of the most common organisms present. Ecologically, echinoderms are often very important as predators or algal grazers. A well known example of the latter is the formation of "urchin barrens", i.e. areas devoid of algae due to intensive grazing by urchins (Gagnon et al. 2003).

Many echinoderms are known to form symbioses with microorganisms, and most of these symbioses are hypothesized to be nutritional in function. For example, sulphideoxidizing bacteria, which are assumed to provide their host with chemosyntheticallyfixed $\mathrm{CO}_{2}$, have been found in the gut of the deposit-feeding urchin Echinocardium cordatum (Temara et al. 1993). Urchins can also benefit from association with bacteria which provide limiting nutrients. An example is Strongylocentrotus droebachiensis, an urchin that contains nitrogen-fixing bacteria in its gut (Guerinot \& Patriquin 1981). There are numerous other examples of echinoderm-associated bacteria which are assumed to play a role in the nutrition of the host, although often their exact function or importance to the host is not clear. Examples include bacteria from the guts of echinoids (De Ridder 
\& Jangoux 1985, Thorsen 1999) and a holothuroid (Deming \& Colwell 1982), and the connective tissue of a crinoid (Holland et al. 1991). The most common symbiosis between echinoderms and microorganisms is that between members of all five echinoderm classes and bacteria living within their sub-cuticular space ("sub-cuticular bacteria", or SCB) (Holland \& Nealson 1978). It is this symbiosis which is the focus of the present study, and which will be reviewed in the following section.

\section{Sub-cuticular bacteria}

Sub-cuticular bacteria (SCB) were first described by Holland and Nealson (1978), although they had previously been observed, but not positively identified as bacteria, by Souza Santos and Sasso (1970). SCB live within the sub-cuticular space of many echinoderms. The sub-cuticular space of echinoderms is bounded on one side by the outer cuticle, and on the other by the epidermal layer of the animal; it varies in size and regularity, generally being a few micrometres in width (although it can be up to $12 \mu \mathrm{m}$ (Bosch 1992)), and sometimes penetrated by microvilli of the epidermal cells (Holland \& Nealson 1978, Walker \& Lesser 1989, Grimmer \& Holland 1990, Lesser \& Blakemore 1990, Kelly et al. 1995, Kelly \& McKenzie 1995). Figures 1.1 and 1.2 show the epidermal layer, sub-cuticular space and cuticle of Odontaster benhami (Asteroidea) and Amphipholis squamata (Ophiuroidea), respectively. In both figures, numerous SCB can also be seen. 


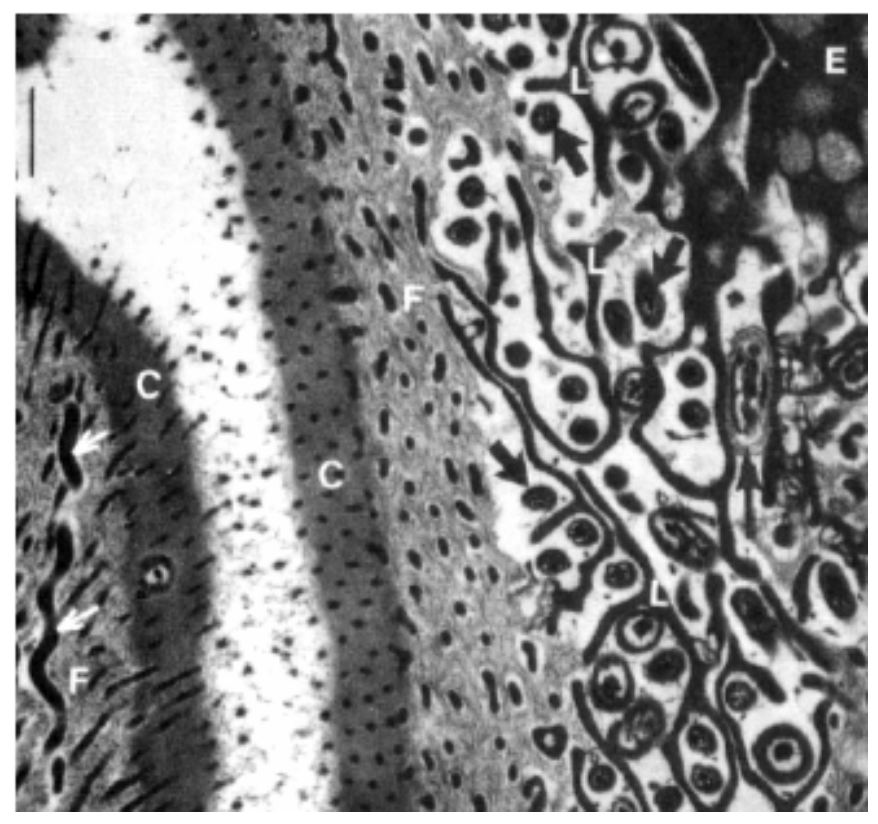

Figure 1.1. Transmission electron micrograph of the asteroid Odontaster benhami tube foot, showing the outer layer of the cuticle (C), the inner fibrous layer of the cuticle (F), the epidermal support cells $(\mathrm{E})$, the lamellae of support cells (L), and numerous SCB (arrows). Scale bar represents $1 \mu \mathrm{m}$. Figure reproduced from Kelly et al. (1995).

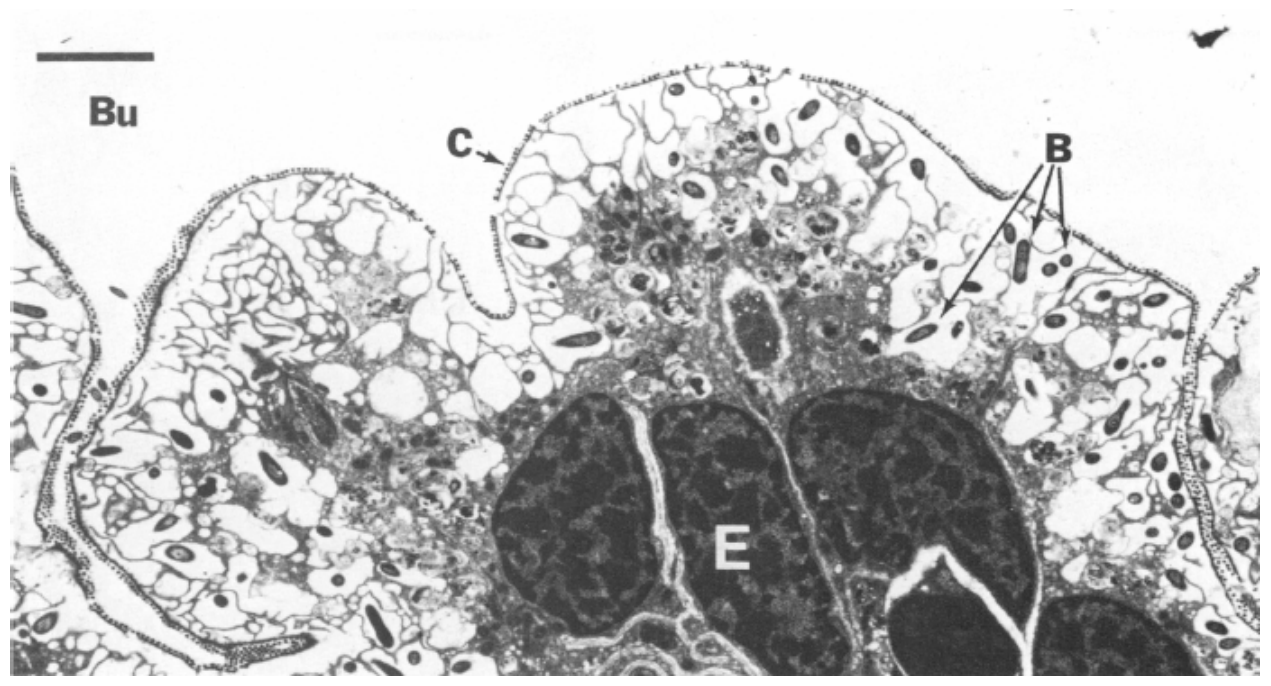

Figure 1.2. Transmission electron micrograph of the bursal area of the ophiuroid Amphipholis squamata, showing the cuticle (C), epidermal cells (E), and SCB (B). Scale bar represents $3 \mu \mathrm{m}$. Figure reproduced from Lesser and Blakemore (1990). 
SCB have been identified as gram-negative bacteria, based on the presence of two membranes surrounding the cytosol (Holland \& Nealson 1978, Roberts et al. 1991, Bosch 1992, McKenzie \& Kelly 1994, Kelly et al. 1995, Kelly \& McKenzie 1995), negative gram-staining of cultured colonies (Lesser \& Blakemore 1990), and the presence of endotoxin, which was determined using the Limulus amoebocyte lysate (LAL) assay (Kelly \& McKenzie 1992, McKenzie \& Kelly 1994) (endotoxin is a component of gramnegative membranes).

SCB have been divided into three types, based on morphology as seen in transmission electron microscopy (TEM) (McKenzie \& Kelly 1994). While this initial grouping was based on observations from ophiuroids, it has since been shown that all known SCB can be classified into one of these three groups. The three types are defined as follows:

Type 1 SCB are small ( $1 \mu \mathrm{m}$ long) rods which are found as pairs of bacteria sharing the same outer capsule. The capsule consists of a complex bi- or tri-laminar outer coat, the layers of which resemble plasma membranes, and beneath the outer coat lie the actual plasma membranes. Internal specialization, such as the presence of vacuoles, is generally not seen in Type 1 cells (McKenzie \& Kelly 1994, Kelly et al. 1995, Kelly \& McKenzie 1995). Figure 1.3 shows typical Type 1 SCB.

Type 2 SCB are characteristically thin, rod-shaped bacteria which, like Type 1 SCB, lack obvious internal specialization. SCB of this type are usually spiral-shaped, but range from 
completely straight rods to tightly-coiled spirals, with intermediate forms resembling loosely-coiled spirals also existing. The shape of Type 2 SCB appears to be influenced by their microhabitat, with those found in confined spaces such as the inner cuticle tending to be straight. These SCB are usually $\sim 1-2 \mu \mathrm{m}$ in length, and $0.1-0.2 \mu \mathrm{m}$ in width. Type 2 SCB are the most commonly seen in all classes of echinoderm (McKenzie \& Kelly 1994, Kelly et al. 1995, Kelly \& McKenzie 1995). Figure 1.4 shows typical Type 2 SCB.

Type 3 SCB are large, straight rods $(\sim 1-2 \mu \mathrm{m}$ long, $0.2-0.5 \mu \mathrm{m}$ wide), with thin, simple capsules. These bacteria often contain membrane-bound vacuoles, unlike Types 1 and 2 which lack internal specialization (McKenzie \& Kelly 1994, Kelly et al. 1995, Kelly \& McKenzie 1995). Type 3 SCB can be further divided into three subtypes. Subtype 1 is that originally described by McKenzie and Kelly (1994); Subtype 2 contains more vacuoles than Subtype 1; Subtype 3 contains no obvious vacuoles and has a well-defined periplasmic layer between the inner and outer plasma membranes. Figure 1.5 shows typical Type 3 (Subtype 3) SCB. 


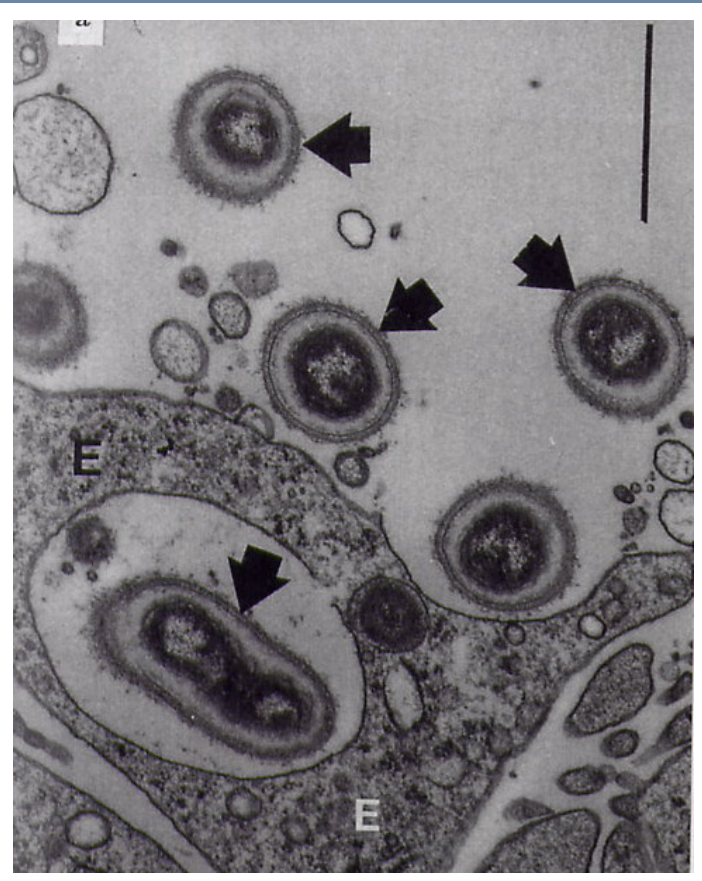

Figure 1.3. Transmission electron micrograph showing the Type 1 SCB (arrows) present in Ophiothrix fragilis (Ophiuroidea). All are shown in transverse section except for the lower bacterium which appears to be in a vacuole of the host epidermal support cell (E). Scale bar represents $1 \mu \mathrm{m}$. Figure reproduced from Kelly and McKenzie (1995).

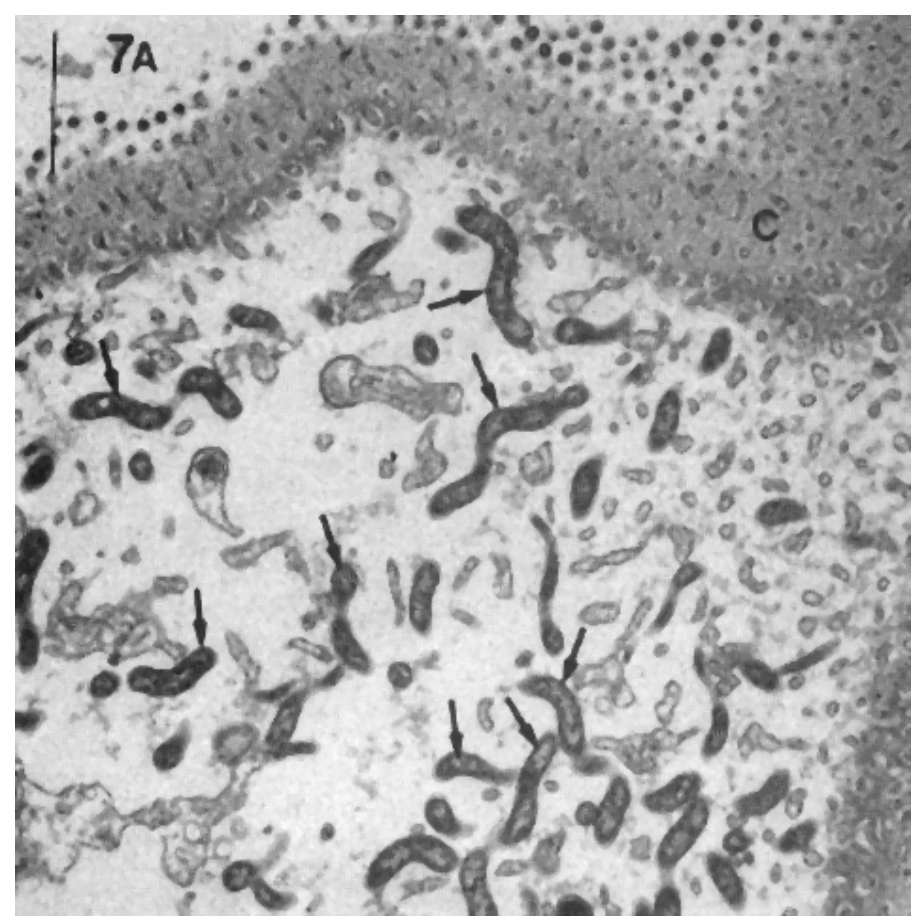

Figure 1.4. Transmission electron micrograph showing the Type 2 SCB present in Astrobrachion constrictum (Ophiuroidea). Note the characteristic spiral shape of those in longitudinal section. Scale bar represents $1 \mu \mathrm{m}$. Figure reproduced from Kelly et al. (1995). 


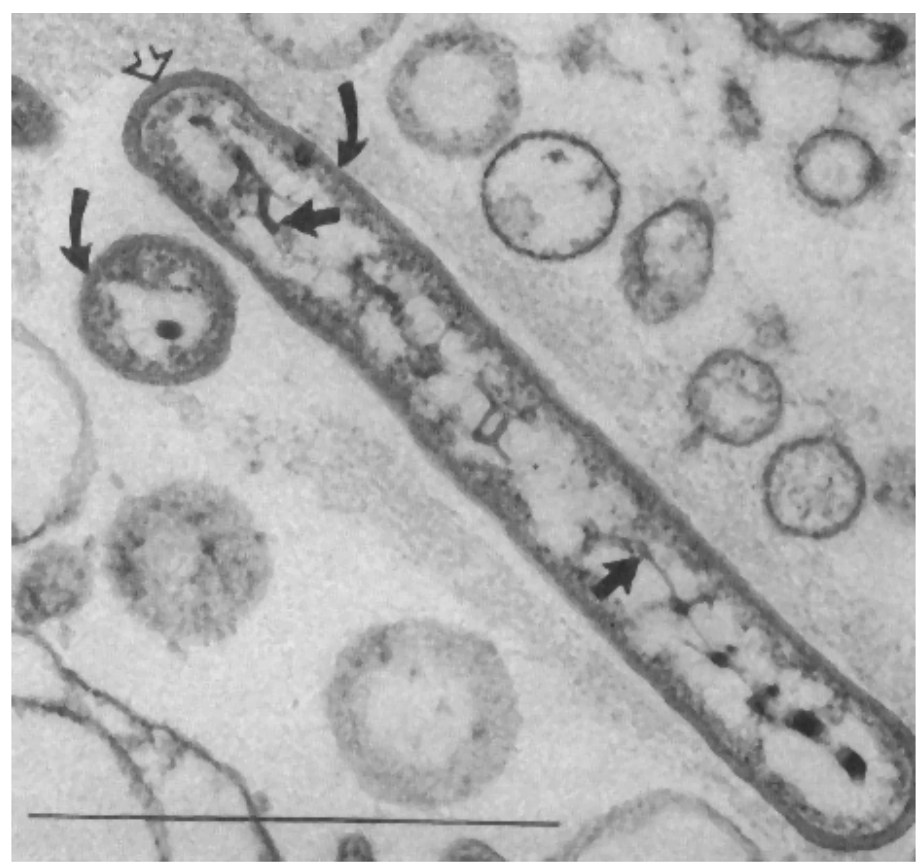

Figure 1.5. Transmission electron micrograph of the Type 3 (Subtype 3) SCB present in Pseudechinus huttoni (Echinoidea). A transverse and longitudinal section can be seen. Arrows inside the bacterium show the electron-dense chromatin fibres running along the centre of the cell. Scale bar represents $1 \mu \mathrm{m}$. Figure reproduced from Kelly et al. (1995).

At this stage it is unknown whether the different morphological types of SCB, and the variations in morphology seen within each type, are indicative of different bacteria (i.e. genotypic differences), or whether they represent phenotypic variation only, perhaps in response to the different environments present within and among echinoderm hosts (McKenzie \& Kelly 1994, Kelly et al. 1995, Kelly \& McKenzie 1995). Only one study to date has reliably identified SCB phylogenetically. Burnett and McKenzie (1997) sequenced part of the 16S rRNA gene of the SCB from the ophiuroid Ophiactis balli, and found that the symbiont is likely an $\alpha$-proteobacterium, closely related to the rhizobia and agrobacteria. An earlier study identified the SCB of the ophiuroid Amphipholis squamata as a member of the genus Vibrio (a $\gamma$-proteobacterium), based on growth requirements, antibiotic sensitivity and the moles percent guanine plus cytosine of its DNA (Lesser \& 
Blakemore 1990). Doubt has been cast on this finding, however, as subsequent workers have been unable to successfully repeat the experiment, and the Vibrio sp. identified is considered more likely to be a contaminant than a SCB (Kelly \& McKenzie 1995).

\section{Distribution of SCB}

SCB are geographically and ecologically widespread. They are found in brittle stars from intertidal mussel beds (Lesser \& Walker 1992), sea cucumbers from depths of over 4,000 m (Roberts et al. 1991), and numerous echinoderms and habitats in between. Although not ubiquitous in echinoderms, SCB are found in all five classes and in the majority of species so far examined (McKenzie et al. 1998). In two of the largest studies of SCB distribution to date, 40 out of 63 echinoderms ( $63 \%)$ from the British Isles and 17 out of 33 echinoderms $(\sim 52 \%)$ from New Zealand were found to contain SCB (Kelly et al. 1995, Kelly \& McKenzie 1995). McKenzie et al. (1998) compiled data from 149 echinoderm species from varying locations, and found that $\sim 60 \%$ contained SCB. Prevalence of SCB presence is not uniform among the different classes: the percentage of species containing SCB ranges from $\sim 30 \%$ in the Crinoidea to $\sim 80 \%$ in the Ophiuroidea (McKenzie et al. 1998). To some extent, this variation is likely caused by insufficient sampling, as data for some groups comes from very few species. However, a closer inspection of the distribution of SCB shows that there is in fact a link between SCB presence and host phylogeny, with certain genera and families consisting almost entirely of SCB-containing species, and others containing very few or no SCB-containing species. Generally speaking, the following associations can be made between SCB presence and host identity in the different echinoderm classes (McKenzie et al. 1998): 
Class Asteroidea: SCB presence may relate to order (an exception is the order Forcipulatida, in which some species contain SCB and some do not).

Class Crinoidea: Insufficient samples have so far been examined to comment with any certainty, but it appears SCB presence may relate to family.

Class Echinoidea: SCB presence is likely related to family.

Class Holothuria: There is no apparent relationship between SCB presence and host identity.

Class Ophiuroidea: In the brittle stars (order Ophiurida), SCB presence seems to be related to family. Very few basket stars (order Phrynophiurida) have been examined, therefore no conclusions can be drawn as to the relationship between SCB presence and host identity. However, the five species examined so far all contain SCB, and these include three species from the same family.

Two hypotheses arise from the finding of a relationship between SCB presence and host phylogeny: 1) Closely related echinoderms are likely to be similar in terms of physiology, habitat and lifestyle, therefore the relationship between SCB presence and host phylogeny exists because SCB presence is related to host ecology. 2) There is a genetic component to the distribution of SCB, most likely involving co-evolution of host and symbiont. The first hypothesis has been tested and falsified. The large data set of McKenzie et al. (1998) shows no relationship between SCB presence and host ecology. A good example is provided by Kelly and McKenzie (1995), who describe the two urchins Psammechinus miliaris and Echinus esculentus. Both species live in similar habitats, and have similar lifestyles, diets and life-history strategies. Despite the apparently high degree of 
similarity between $P$. miliaris and E. esculentus, only one (P. miliaris) contains SCB. With no apparent relationship between SCB presence and host ecology, but a link between SCB presence and host phylogeny, it seems likely that the echinoderm-SCB symbiosis is an ancient relationship and an example of co-evolution.

\section{Abundance of SCB}

Several methods have been used to determine the abundance of SCB in echinoderms, however the most commonly used is direct counting of SCB using epifluorescence microscopy. Briefly, this method involves homogenization of a known quantity of echinoderm tissue, staining with a fluorescent dye such as acridine orange, and visualization using an epifluorescence microscope. To translate the observed quantity of SCB into an estimate of SCB load in a given echinoderm, wet weight and ash-free dry weight (AFDW, determined by drying tissue samples, weighing them, then incinerating them in a muffle furnace to establish the proportion of the weight made up of ash) are calculated for the echinoderms under study. The bacterial loads (expressed as SCB $\mathrm{g}^{-1}$ AFDW host tissue) of all SCB-containing echinoderms which have been examined in this way are presented in Table 1.1. As can be seen in this table, there is considerable variation in SCB load between host species (greater than one order of magnitude), however values are generally very high $\left(10^{8}-10^{9} \mathrm{SCB}^{-1}\right.$ AFDW host tissue). Such values suggest that SCB may be important to those echinoderms which possess them, as similar values have been recorded from bacteria in the tube worm Riftia pachyptila, an animal which lacks a gut and derives its energy entirely from its chemoautotrophic bacterial symbionts (Cavanaugh et al. 1981). In addition to interspecific variation in SCB 
load, significant intraspecific variation occurs, both spatially (e.g. between different populations of the same echinoderm species) and temporally (e.g. in the same population at different times of the year) (McKenzie \& Kelly 1994, Kelly et al. 1995, McKenzie et al. 2000, Foret \& Lawrence 2001). Examples include the ophiuroid Ophiophragmus filograneus, in which SCB loads were found to range from $2.80 \times 10^{8}-2.78 \times 10^{9} \mathrm{~g}^{-1}$ AFDW host tissue between different populations, and the asteroid Luidia clathrata, in which SCB loads varied from $3.8 \times 10^{8} \mathrm{~g}^{-1}$ AFDW host tissue in January to $7.9 \times 10^{8} \mathrm{~g}^{-1}$ AFDW host tissue in September (Foret \& Lawrence 2001). Currently the reason for this variation is not known for certain, however there is some suggestion that reduced SCB load may be a consequence of stress to the host, SCB or both. For example, Newton and McKenzie (1995) found reduced SCB numbers in the ophiuroids Amphiura filiformis, A. chiajei and Ophiothrix fragilis in response to hydrocarbon insult. Echinoderms kept in static seawater were also found to have a reduced SCB load compared to those kept in through-flow systems, again suggesting a stress-induced loss of SCB (Kelly \& McKenzie 1992, Newton \& McKenzie 1998). It has been suggested that this response could be used as an assay for environmental stress, such as oil pollution (Newton \& McKenzie 1995), however such assays would obviously need to be used with caution, given the large intraspecific variation in SCB load seen across time and space (Foret \& Lawrence 2001). 
Table 1.1. SCB loads of symbiotic echinoderms.

\begin{tabular}{|c|c|c|c|}
\hline Host species & Host Class & SCB g $^{-1}$ AFDW* & Reference \\
\hline Anseropoda placenta & Asteroidea & $3.58 \times 10^{9}$ & Kelly and McKenzie (1995) \\
\hline Asterodon miliaris & Asteroidea & $1.99 \times 10^{9}$ & Kelly et al. (1995) \\
\hline Luidia clathrata & Asteroidea & $6.40 \times 10^{8}$ & Foret and Lawrence (2001) \\
\hline Arbacia punctulata & Echinoidea & $2.57 \times 10^{8}$ & Foret and Lawrence (2001) \\
\hline Lytechinus variegates & Echinoidea & $1.45 \times 10^{8}$ & Foret and Lawrence (2001) \\
\hline Mellita tenuis & Echinoidea & $7.93 \times 10^{8}$ & Foret and Lawrence (2001) \\
\hline Psammechinus miliaris & Echinoidea & $1.16 \times 10^{9}$ & Kelly and McKenzie (1995) \\
\hline Pseudechinus albocinctus & Echinoidea & $2.17 \times 10^{9}$ & Kelly et al. (1995) \\
\hline Pseudechinus huttoni & Echinoidea & $4.48 \times 10^{9}$ & Kelly et al. (1995) \\
\hline Pseudechinus huttoni juveniles & Echinoidea & $2.67 \times 10^{9}$ & Kelly et al. (1995) \\
\hline Pseudechinus novaezealandiae & Echinoidea & $8.41 \times 10^{8}$ & Kelly et al. (1995) \\
\hline \multirow[t]{2}{*}{ Amphipholis squamata } & Ophiuroidea & $4.96 \times 10^{9}$ & Kelly et al. (1995) \\
\hline & & $4.60 \times 10^{9}$ & McKenzie and Kelly (1994) \\
\hline Amphiura chiajei & Ophiuroidea & $1.69 \times 10^{9}$ & McKenzie and Kelly (1994) \\
\hline Amphiura filiformis & Ophiuroidea & $2.00 \times 10^{9}$ & McKenzie and Kelly (1994) \\
\hline Ophiactis balli & Ophiuroidea & $3.02 \times 10^{9}$ & McKenzie and Kelly (1994) \\
\hline Ophiocoma bollonsi & Ophiuroidea & $4.41 \times 10^{8}$ & Kelly et al. (1995) \\
\hline Ophiopholis aculeata & Ophiuroidea & $6.2 \times 10^{8}$ & McKenzie and Kelly (1994) \\
\hline Ophiophragmus filograneus & Ophiuroidea & $1.79 \times 10^{9}$ & Foret and Lawrence (2001) \\
\hline \multirow[t]{2}{*}{ Ophiothrix fragilis } & Ophiuroidea & $1.32 \times 10^{9}$ & Kelly and McKenzie (1995) \\
\hline & & $1.33 \times 10^{9}$ & McKenzie and Kelly (1994) \\
\hline Ophiura albida & Ophiuroidea & $1.54 \times 10^{9}$ & McKenzie and Kelly (1994) \\
\hline Ophiura ophiura & Ophiuroidea & $3.70 \times 10^{8}$ & McKenzie and Kelly (1994) \\
\hline
\end{tabular}

* All SCB loads listed above are mean values. Intraspecific variation in SCB abundance was found to often be significant (up to an order of magnitude). 


\section{Mode of transmission of SCB}

It is not known whether SCB are transmitted vertically (i.e. passed from echinoderm parent to offspring) or horizontally (i.e. taken up from seawater by each generation of the host echinoderm). Observations of SCB in very young echinoderms suggest that, if vertical transmission is not occurring, then infection by free-living bacteria must occur very early in the host's life (Barker \& Kelly 1994). Several lines of evidence favour the vertical transmission hypothesis. The relationship between SCB presence and host phylogeny, and the presumed co-evolution of host and symbiont, implies a high degree of specificity in the symbiosis and therefore vertical transmission (there is a significant risk of not obtaining the correct bacterium from the seawater each generation if horizontal transmission is at work). Related to this is the general observation that mutualistic bacteria, which SCB are thought to be (see next section), tend to be transmitted vertically, while parasitic bacteria tend to be horizontally transmitted (Haine 2008). Furthermore, the presence of SCB in brooded embryos of the ophiuroid Amphipholis squamata is strong evidence for vertical transmission, although whether the same process takes place in other, non-brooding, species remains to be seen (Walker \& Lesser 1989).

Horizontal transmission is not an entirely unlikely scenario, however. Even if the echinoderm-SCB symbiosis is highly specific, infection by free-living bacteria could account for the presence of SCB in host species. This scenario does occur in other marine invertebrate-bacteria associations, for example the mutualism between the squid Euprymna scolopes and the bioluminescent bacterium Vibrio fischeri. In this symbiosis, the bacterium is acquired from seawater by each generation of squid, despite the highly specific nature of the relationship (Ruby 1996, Mcfall-Ngai 1999). Generally speaking, 
extracellular bacterial symbionts of animals (such as SCB) are usually acquired from the environment, whereas intracellular symbionts are normally transmitted vertically (Phillips 2006). Furthermore, Holland and Nealson (1978) point out that bacteria have not been observed on or in echinoderm eggs, which suggests horizontal transmission. These authors go on to suggest that the characteristic SCB community of an echinoderm may change from generation to generation due to differences in the bacterial community composition of the surrounding seawater. Further studies incorporating gene sequencing and phylogenetic analysis are needed before this can be determined one way or the other.

Attempts to empirically determine the mode of transmission, by looking for SCB in various stages of host development, and attempting to infect larvae with SCB from parent tissue have had little success. Therefore, the mode of transmission of SCB remains unresolved.

\section{Functional roles of the echinoderm-SCB symbiosis}

As mentioned previously, SCB abundance is often very high, suggesting that SCB may be important to their hosts. Exactly what purpose they serve, however, has not been established. Currently, the two leading hypotheses are that the SCB provide the host with a nutritional benefit or with antimicrobial protection.

A nutritional benefit could be provided in several ways. Host epidermal cells contain many lysosomes, and SCB in various stages of phagocytosis by host cells have been observed in several echinoderm species, suggesting that a nutritional benefit comes about 
by cropping of SCB by the host (Holland \& Nealson 1978, Walker \& Lesser 1989, Roberts et al. 1991, Bosch 1992, McKenzie \& Kelly 1994). However, it has not been shown whether phagocytosis is in fact a significant source of nutrition, or whether the host only ingests moribund cells, in which case it may represent 'sanitation' rather than 'feeding' per se. That being said, it would not be surprising if the host did ingest SCB for the purpose of gaining energy or limiting nutrients. Bacteria take up and concentrate dissolved nutrients more efficiently at the low levels found in the ocean than metazoans such as echinoderms do, meaning phagocytosis of SCB could be an effective way of obtaining such nutrients (Feral 1985, Walker \& Lesser 1989, Roberts et al. 1991, Bosch 1992). An alternative route for incorporation of bacterially assimilated dissolved nutrients may be through pinocytosis, i.e. the uptake by host epidermal cells of nutrients or metabolic by-products which are released from the SCB (Holland \& Nealson 1978, Feral 1980, Walker \& Lesser 1989). Unfortunately, research into the echinoderm-SCB symbiosis has largely relied on microscopy, so the actual benefit to the host from phagocytosis or pinocytosis has yet to be established.

There are, of course, other ways in which SCB may provide their hosts with a nutritional benefit. The possibility exists that SCB are chemosynthetic and provide their hosts with energy, but this would be unusual given that they are found in echinoderms from such a wide range of environments, many of which do not seem conducive to chemotrophy, and that echinoderms have fully-functioning guts, unlike other invertebrates which rely on chemotrophic bacteria for energy. Alternatively, SCB may be involved in nitrogen fixation. Many echinoderms are grazers, with relatively low-nitrogen diets, and nitrogen- 
fixing bacteria have been found in the gut of the echinoid Strongylocentrotus droebachiensis (Guerinot \& Patriquin 1981). Additionally, the SCB identified in Ophiactis balli fall within the $\alpha-2$ subdivision of the $\alpha$-proteobacteria, along with the nitrogen-fixing rhizobacteria (Burnett \& McKenzie 1997).

While the hypothesized nutritional benefits of SCB to their hosts are based mostly on assumptions from microscopic observations and comparisons with other invertebratebacteria symbioses, some empirical evidence does exist. Lesser and Walker (1992) examined the uptake of dissolved free amino acids (FAA) from seawater, and their subsequent incorporation into protein, by two species of ophiuroid, one of which contained SCB (Amphipholis squamata) and one of which was thought not to (Ophiopholis aculeata). Their results showed that bacteria did play a role, albeit small, in FAA uptake, accounting for less than $10 \%$ of total uptake. The incorporation of FAA into protein was found to be carried out primarily by $\mathrm{SCB}$, as A. squamata treated with chloramphenicol (an antibiotic) showed an $89 \%$ decrease in FAA incorporation. The results of this study must be treated with caution, as the non-symbiotic O. aculeata was later found to contain SCB, albeit at a density almost an order of magnitude lower than that of A. squamata (McKenzie \& Kelly 1994) (See Table 1.1 for SCB densities of both species). While this does not affect the finding of a high level of bacterial incorporation of FAA into protein, the comparison of FAA uptake between the two hosts may be misleading, as SCB may in fact be playing a greater part in FAA uptake than the results suggest. 
McKenzie et al. (2000) used fatty acid and stable isotope analysis to determine the nutritional role of SCB in the ophiuroids Ophiothrix fragilis and Amphiura chiajei. Carbon isotope values were in the range expected for organisms feeding on phytoplankton, implying that SCB are heterotrophic. A negative correlation was observed between $\delta^{15} \mathrm{~N}$ values and SCB load, suggesting that SCB supply their hosts with a significant amount of nitrogen. The fatty acids $16: 1 \omega 7$ and 18:1 17 were found at relatively high levels in SCB-containing echinoderms, suggesting that SCB also contribute to the fatty acid pool of their hosts.

The hypothesized antimicrobial action of SCB arises from observations that SCB can rapidly colonize echinoderm wound sites (such as ophiuroid arm tips, which are easily damaged), and could presumably exclude colonization by water-borne bacteria (McKenzie \& Kelly 1994). The anti-fouling ability of echinoderms is well known (McKenzie \& Grigolava 1996, Guenther et al. 2007), and it may be the case that this ability arises from the presence of SCB in some echinoderm species. Whether this hypothesized antimicrobial action is achieved through competitive exclusion or production of antimicrobial compounds by SCB is unknown. Strahl et al. (2002) have shown that extracts from echinoderm-associated bacteria have antibacterial activity against a range of potentially pathogenic bacteria (their work involved streaking the bacterial isolates on Zobell modified 2216E agar, then applying various test bacteria and observing the level of inhibition of these test bacteria in response to the different isolates). By the authors' own admission, however, it is not known which isolates were obtained 
from SCB and which were from other echinoderm-associated bacteria. Therefore, the mode of antimicrobial action by SCB, if indeed it does occur, remains unknown.

Other functions of SCB have been suggested; for example Holland and Nealson (1978) note that several species of echinoderm are known to be bioluminescent and that it is possible that SCB are responsible in some cases. This seems unlikely, though, as SCB have been found in many echinoderms in the years since their study was published, and the majority do not appear to be bioluminescent. Alternatively, SCB could be sulphuroxidizing bacteria. Many echinoderms, particularly burrowing urchins and sea cucumbers, live in potentially sulphide-rich environments. As noted by Kelly and McKenzie (1995), structures seen in transmission electron micrographs of SCB, which are generally presumed to be poly- $\beta$-hydroxybutyrate bodies, could well be sulphur inclusions, indicating that the SCB are sulphur-oxidizing bacteria.

The benefit to the SCB from this presumably mutualistic relationship is unclear at this stage. In larvae of the asteroid Luidia sp. SCB abundance was highest in regions of the sub-cuticular space nearest to the host's stomach, suggesting that SCB may benefit from byproducts of the host's digestion (Bosch 1992). A similar benefit was proposed by Holland and Nealson (1978), who pointed out that SCB, which are in close proximity to host epidermal cells, may take up dissolved organic materials leaked from the host. Alternatively, the benefit to the SCB may be in the form of a safe place to live, which is exposed to high concentrations of dissolved organic matter, especially in burrowing echinoderms (Roberts et al. 1991). 
Clearly, there are large gaps in our knowledge of the echinoderm-SCB symbiosis. Important areas which need to be addressed include identifying the SCB from as many echinoderms as possible, determining cultivation requirements of SCB in order to study the symbionts in more detail, and further attempts at empirically testing the function of the symbiosis. The present study addresses the first two points, by attempting to phylogenetically identify and cultivate the SCB of several common New Zealand echinoderms. The exact aim of this study and the approaches used are presented in the following section.

\subsection{Aim and specific objectives}

The aim of this study was to characterize the SCB of common New Zealand echinoderms by molecular means. Specific objectives were:

1. To determine the phylogenetic identity of the SCB of several common New Zealand echinoderms.

2. To provide further evidence for the presence or absence of SCB in these echinoderms using fluorescence in situ hybridization (FISH).

3. To cultivate the SCB found using the above methods.

The thesis is divided into five chapters. Chapter 2 presents the results of experiments designed to determine the phylogenetic identity of SCB. Three shallow-water, and four deep-water echinoderms were examined by sequencing a region of the $16 \mathrm{~S}$ rRNA genes 
of their associated bacteria, and phylogenetic analysis was carried out to determine their approximate identity. In Chapter 3, results of FISH assays are presented. FISH is a technique which uses fluorescently-labeled oligonucleotide probes of varying target specificity to search for the presence of bacteria in situ. FISH assays were performed on the three shallow-water echinoderm species that were analyzed in Chapter 2. The results of SCB cultivation attempts are presented in Chapter 4. Attempts at cultivation were carried out using two of the shallow-water echinoderms which were found to contain SCB. A general discussion is provided in Chapter 5, which includes a summary of results, consideration of the potential functions of the putative SCB identified, and future research directions.

Notes:

Throughout this thesis, Patiriella sp. is used to refer to the cushion star formerly known as Patiriella regularis. Based on molecular and morphological analyses, $P$. regularis has been split into two species: P. regularis and P. mortenseni (O'Loughlin et al. 2002). However, due to difficulties in distinguishing the two species, the cushion stars examined in the present study are grouped together as Patiriella sp.

Chapters $2-4$ are written as individual scientific papers; consequently there is overlap of material between chapters, particularly in the introductions. 


\title{
Chapter 2: Phylogenetic identification of sub-cuticular bacteria
}

\begin{abstract}
Many echinoderms contain symbiotic bacteria that live in the sub-cuticular space, between the epidermis and outer cuticle. These so-called sub-cuticular bacteria (SCB) are gram-negative bacilli of unknown function. There is a lack of knowledge regarding the identity of SCB; the only phylogenetic information to date comes from a brittle star, and suggests that SCB are members of the $\alpha$-proteobacteria. In this study, PCR and sequencing of $16 \mathrm{~S}$ rRNA genes and phylogenetic analysis were used to determine the phylogenetic affiliations of the SCB belonging to a range of echinoderm species. Four putative SCB were identified, all of which were found in association with the sea cucumber Stichopus mollis (Class Holothuroidea), and two of which were found in association with the sea star Patiriella sp. (Class Asteroidea). Of these four bacteria, two belong to the $\alpha$ subdivision of the proteobacteria, one belongs to the $\gamma$ subdivision, and one falls among members of both the $\alpha$ and $\gamma$ subdivisions. The nearest relatives of these four species are varied: some are symbionts of marine invertebrates, others are symbionts of terrestrial organisms, and others are pathogens and parasites of various organisms. If the bacteria identified here are in fact SCB, the wide variety of closely related bacteria raises interesting questions about the nature of this symbiosis.
\end{abstract}




\subsection{Introduction}

Sub-cuticular bacteria (SCB) are symbionts that live between the epidermis and the cuticle (the sub-cuticular space) in a range of echinoderms. Despite first being described by Holland and Nealson in 1978, little is known about this symbiosis, such as the identity of the bacterial symbionts, their mode of transmission into the sub-cuticular space, or what benefit, if any, the echinoderms or bacteria gain from the relationship. Previous work has relied mainly on microscopy to characterize SCB by morphological means (Feral 1980, Lesser \& Blakemore 1990, Roberts et al. 1991, McKenzie \& Kelly 1994, Kelly et al. 1995, Kelly \& McKenzie 1995). Such studies have been useful in determining which echinoderms contain SCB and how abundant the bacteria are within their hosts, and have gone some way towards identification of SCB based on their morphology. One clear trend which has emerged is the prevalence of this symbiosis. In two of the more extensive studies to date, Kelly and co-workers (Kelly et al. 1995, Kelly \& McKenzie 1995), studying a range of echinoderms from all five major classes in Great Britain and New Zealand, found that more than half contained SCB. These researchers and others (e.g. (Lesser \& Blakemore 1990, Roberts et al. 1991, McKenzie \& Kelly 1994)), have identified SCB as rod-shaped, gram-negative bacteria. Three different types of these rod-shaped bacteria have been described: Type 1, which are pairs of bacteria sharing an outer capsule; Type 2, which are curved or spiral-shaped rods approximately 1-2 $\mu \mathrm{m}$ in length; and Type 3, which are similar to Type 2, but thicker and generally straighter (McKenzie \& Kelly 1994). All of the SCB studied thus far can be assigned to 
one of these types (Kelly \& McKenzie 1995), suggesting similarity in the identity of SCB among different hosts.

Not only are SCB widespread among echinoderms, but they are also abundant within the host tissue. Varying values have been reported, but even the lowest densities, in the order of $>10^{7}$ SCB cells $\mathrm{g}^{-1}$ ash free dry weight (Foret \& Lawrence 2001) suggest that SCB may be of importance to those species which possess them. The potential importance of this symbiosis becomes even more apparent if we look at the SCB abundances reported by Kelly and McKenzie (Kelly \& McKenzie 1995), which in some echinoderms were $>10^{9} \mathrm{SCB}$ cells $\mathrm{g}^{-1}$ ash free dry weight, a similar value to that of chemoautotrophic bacteria in the vestimentiferan tube worm Riftia pachyptila, which are assumed to be important in supplying energy to their host (Cavanaugh et al. 1981).

While microscopic studies have yielded interesting information about the echinodermSCB symbiosis, the knowledge we can obtain from such work is limited. Traditionally, culturing has been an important part of research into bacterial biology, but recently the vast majority of bacteria have been shown to be uncultivable using conventional methods (Hugenholtz 2002). Previous attempts at culturing SCB from Ophiuroids have produced equivocal results. Walker and Lesser (1989), and Lesser and Blakemore (1990) claimed to have successfully cultured SCB from Amphipholis squamata and identified them as members of the genus Vibrio. Subsequent work has cast doubt on these findings, as the authors were unable to reproduce the results (Kelly et al. 1995). Whether or not it is 
possible to culture SCB is still open to debate, but it would not be at all surprising if culturing was very difficult.

With the addition of molecular techniques over the past few decades, and the relatively low cost and routine laboratory use of these techniques, it is now possible to garner a substantial amount of information about bacteria such as SCB without the need for culturing. Molecular techniques, such as the polymerase chain reaction (PCR), cloning and sequencing are particularly helpful in terms of identifying bacteria phylogenetically, which in turn can shed light on their physiology, metabolism and relationships with other organisms. In spite of the widespread availability of these techniques, only one study has so far examined SCB using molecular methods (Burnett \& McKenzie 1997). By sequencing a region of the $16 \mathrm{~S}$ rRNA gene from a bacterium isolated from the Ophiuroid Ophiactis balli, Burnett and McKenzie (1997) were able to identify the SCB of this species as an $\alpha$-proteobacterium, closely related to several plant symbionts and pathogens. This finding was particularly interesting as it was the first report of extracellular symbionts belonging to the $\alpha$ subdivision of the proteobacteria. Despite this interesting finding, no further attempts to identify SCB phylogenetically have been made in the eleven years since this study was published.

The present study expands on this previous work by determining whether SCB are present in a range of echinoderm species and, if so, identifying these bacteria using molecular techniques. Three shallow-water echinoderms were studied in depth: Patiriella sp. (class Asteroidea), Stichopus mollis (class Holothuroidea) and Astrostole scabra 
(class Asteroidea). Additionally, preliminary studies were carried out on several deepwater echinoderms: Rosaster mimicus, Psilaster acuminatus, Perissasterias monocantha and Diplopteraster sp. (class Asteroidea). DNA belonging to bacteria associated with these echinoderms was isolated, and part of the 16S rRNA gene was sequenced. Using these sequence data, the phylogenetic affiliations of several potential SCB were determined. The relationship of the putative SCB identified here to previously identified bacteria may offer some insight into the role of SCB in the symbiosis, and some of these potential roles are discussed herein.

\subsection{Materials and methods}

\section{Sample collection and DNA extraction.}

Shallow-water echinoderms. Three species of shallow-water echinoderms were examined for this study: Astrostole scabra (class Asteroidea), Patiriella sp. (class Asteroidea) and Stichopus mollis (class Holothuroidea) (See Figures 2.1 - 2.3). Six individuals of each species were collected from Island Bay, Wellington, New Zealand (latitude $41^{\circ} 20^{\prime} \mathrm{S}$, longitude $174^{\circ} 46^{\prime} \mathrm{E}$ ) (See Figure 2.4) at depths of 2-3 metres and transferred directly to plastic buckets containing seawater. Collecting took place on different days throughout summer (December - February). Echinoderms were rinsed with $1 \mu \mathrm{m}$-filtered seawater (FSW) within two hours of collection and left in FSW overnight, in order to clear their guts and thus reduce the risk of contamination by food-, sedimentor gut-associated bacteria. 
The following day, sections of approximately $5 \times 5 \mathrm{~mm}$ were excised from dorsal surfaces of Patiriella sp. and A. scabra, and both dorsal and ventral surfaces of S. mollis using sterile techniques, with care being taken to avoid penetrating the body cavity. In order to prevent contamination by surface-associated bacteria, the surfaces of the echinoderms were washed for $5 \mathrm{sec}$ in $70 \%$ ethanol followed by two washes in sterile artificial seawater (ASW) prior to sampling. Echinoderms were placed back into $1 \mu \mathrm{m}$ filtered seawater, and 1 hour later wound sites were swabbed with sterile cotton-tipped swabs and another $5 \times 5 \mathrm{~mm}$ tissue section was excised adjacent to the original sampling site, in order to collect any symbiotic bacteria which may have colonized the wound site. Additionally, surface-associated bacteria were collected with sterile cotton-tipped swabs, and water-borne bacteria were collected by filtering $500 \mathrm{ml}$ of FSW through a $47 \mathrm{~mm}$ diameter, $0.22 \mu \mathrm{m}$ pore-size cellulose filter disc (Millipore, Bedford, MA) using a vacuum pump (Note: FSW was only sampled 5 times, not 6 as was the case with all other samples taken). 
Chapter 2: Phylogenetic analysis of SCB

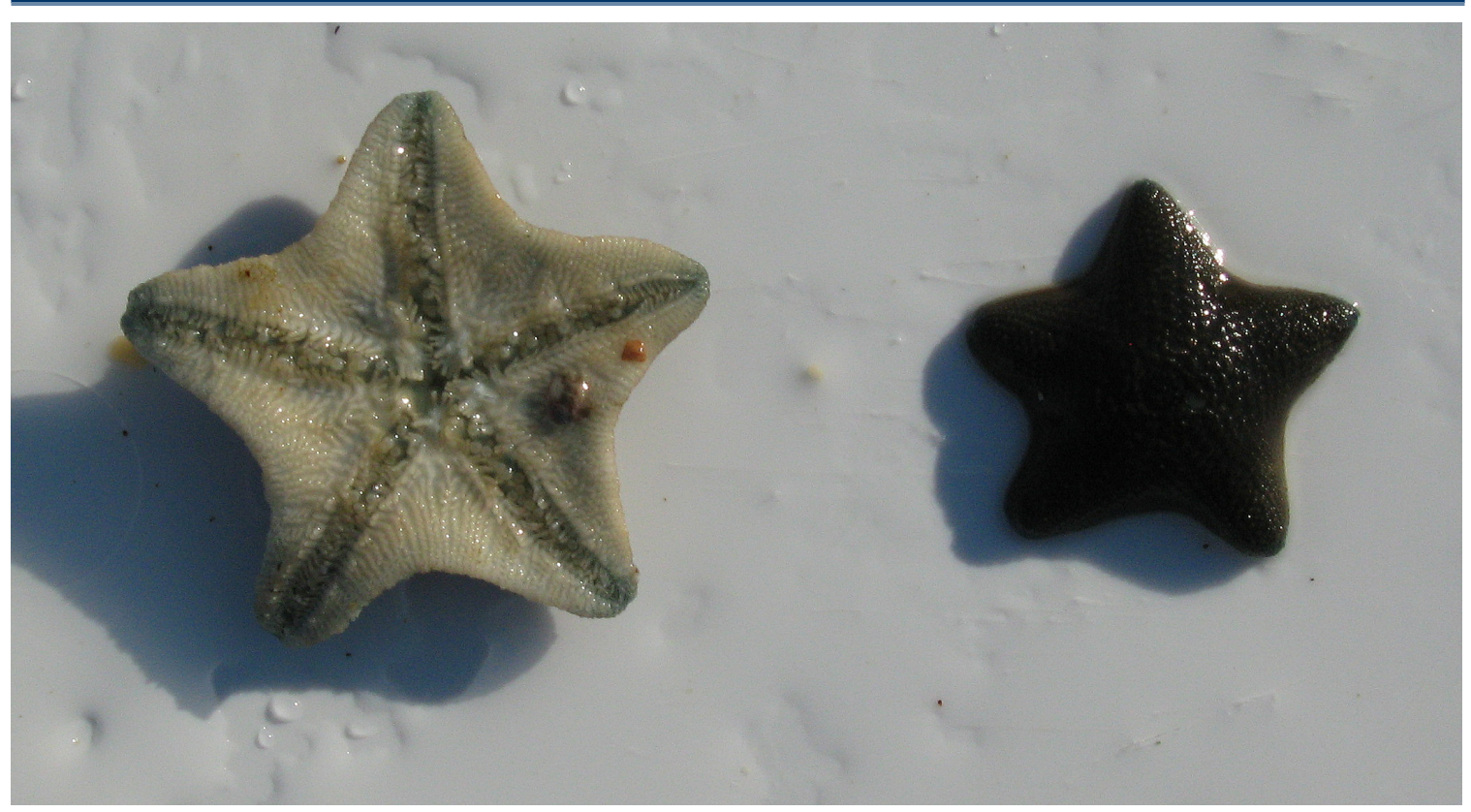

Figure 2.1. Ventral (oral) and dorsal (aboral) views of Patiriella sp.

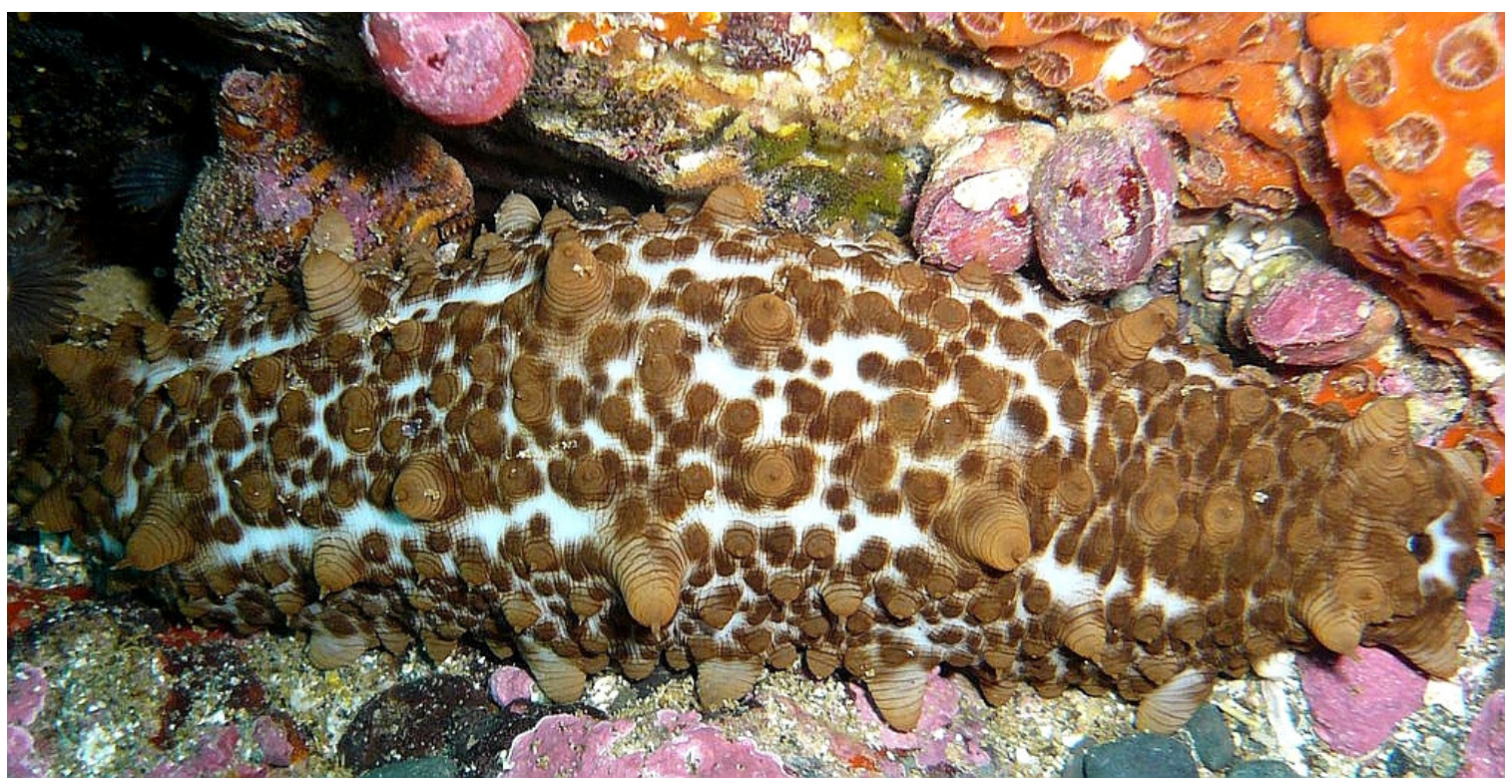

Figure 2.2. Stichopus mollis. Image reproduced from http://www.picasaweb.google.com/akunidivespecies. 


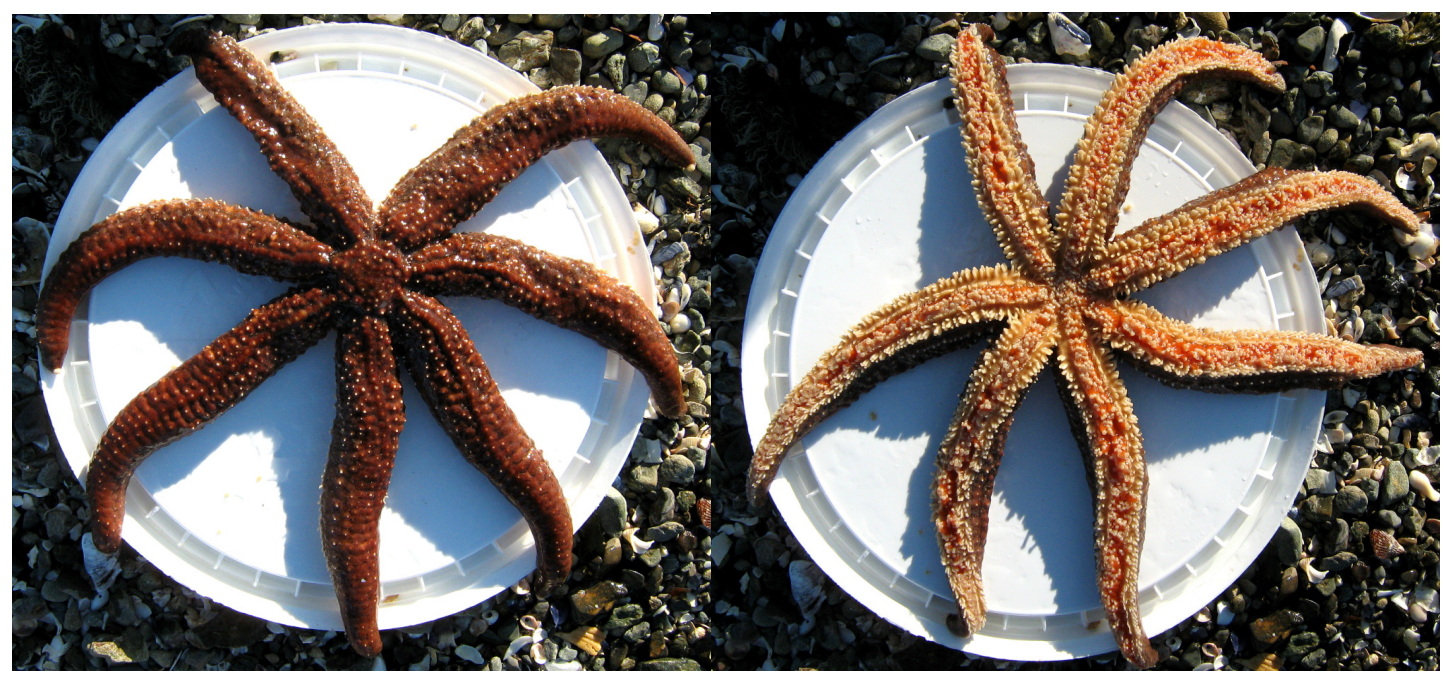

Figure 2.3. Astrostole scabra. A: Dorsal (aboral) view. B: Ventral (oral) view.

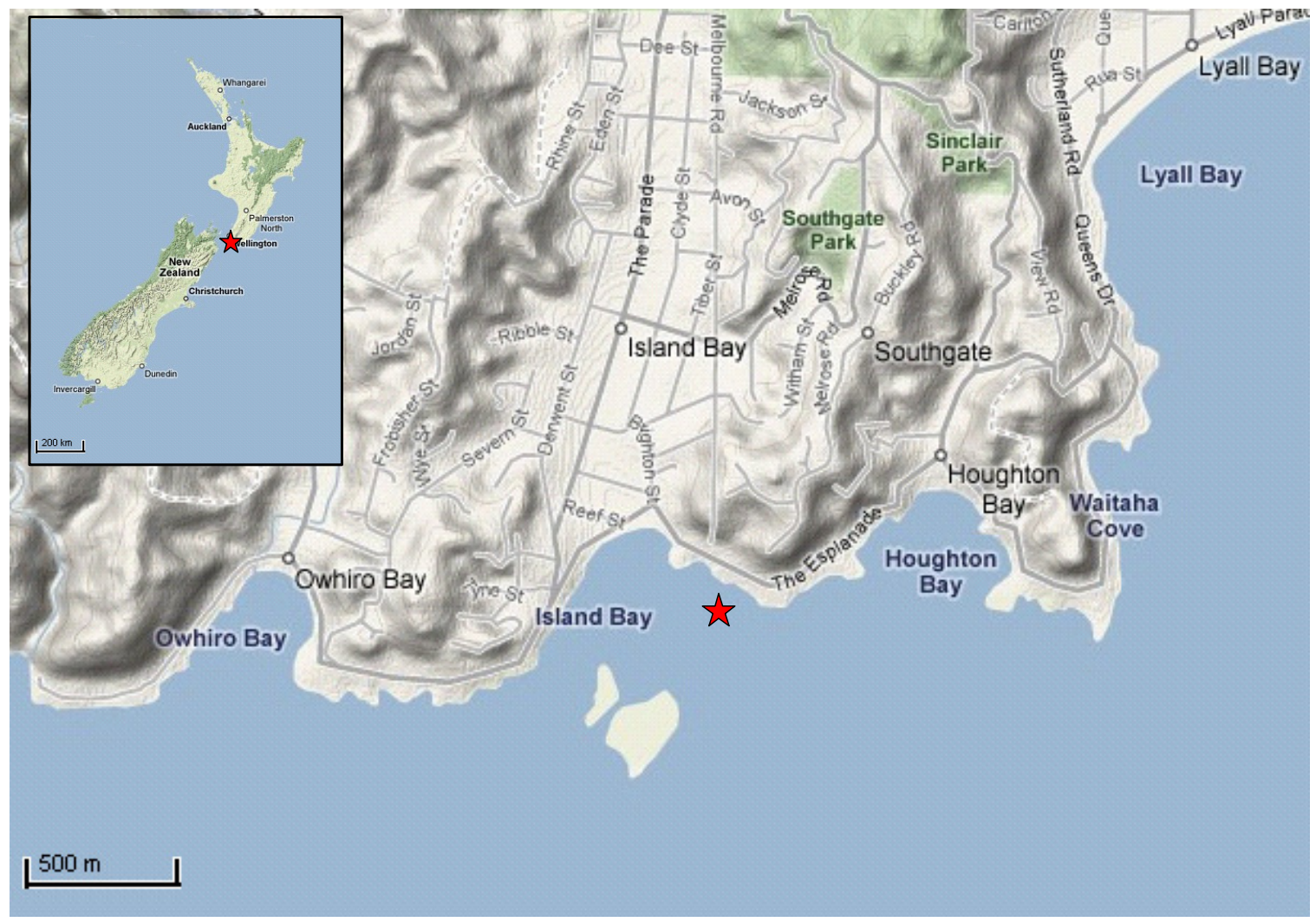

Figure 2.4. Map showing location of shallow-water echinoderm collection (marked with red star). Image reproduced from Google Maps (http://maps.google.co.nz). 
Deep-water echinoderms. Four species of deep-water echinoderms, all belonging to the class Asteroidea, were used in this study: Rosaster mimicus, Psilaster acuminatus, Perissasterias monocantha and Diplopteraster sp. These specimens were collected by the National Institute of Water and Atmospheric Research (NIWA) Ltd. R. mimicus and $P$. acuminatus were collected from seamounts in the Bay of Plenty (latitude $\sim 35^{\circ} \mathrm{S}$, longitude $\sim 179^{\circ} \mathrm{E}$ ) at depths of $312 \mathrm{~m}$ and $232 \mathrm{~m}$ respectively. P. monocantha was collected near the sub-antarctic Snares Islands (latitude $48^{\circ} 01^{\prime} \mathrm{S}$, longitude $166^{\circ} 32^{\prime} \mathrm{E}$ ) and Diplopteraster sp. on the Southern Plateau (latitude $\sim 50^{\circ} \mathrm{S}$, longitude $\sim 170-175^{\circ} \mathrm{E}$ ), both at unrecorded depths. Following collection, specimens were stored at $-20{ }^{\circ} \mathrm{C}$. Tissue samples were excised from the dorsal surface of one individual of each species using a sterile $6 \mathrm{~mm}$-diameter biopsy punch, and from ventral surfaces by removing several tube feet; surface swabs were obtained in the same manner as for the shallow-water species.

Genomic DNA was extracted from tissue samples and surface swabs of all echinoderm species, and filter discs containing concentrated seawater-borne bacteria using a High Pure PCR Template Preparation kit (Roche Applied Science). Extraction was carried out following the manufacturer's instructions, except for the addition and incubation of $5 \mu \mathrm{l}$ lysozyme $\left(10 \mathrm{mg} \mathrm{ml}^{-1}\right.$ in $10 \mathrm{mM}$ Tris-HCl) for $15 \mathrm{~min}$ at $37^{\circ} \mathrm{C}$ following the initial tissue lysis step. 


\section{PCR and cloning}

Bacterial 16S rRNA genes were amplified using the previously published eubacterial forward primer PLK1 (Klaschik et al. 2002) in conjunction with the $\alpha$ - and $\beta$ proteobacteria specific reverse primer ABR1, and the $\gamma$ - and $\delta$-proteobacteria specific reverse primer GDR2. Primers ABR1 and GDR2 were designed from a multiple sequence alignment in the software package Vector NTI Advance 10 (Invitrogen). Several other primers were used in pilot studies, but were deemed unsuitable for a variety of reasons, leading to the selection of the above primers for amplification of bacterial DNA (For a list of primers trialed, and reasons for their disuse, see appendix Table A1). The sequences of the primers used were as follows: PLK1, 5'-TAC GGG AGG CAG CAG T-3'; ABR1, 5'-CCA TGA GGA CTT GAC GTC-3'; GDR2, 5'-TAG CAC GTG TGT AGC CCT-3'. The 16S rRNA gene positions targeted by each primer are as follows (E. coli numbering): PLK1, 343-358; ABR1, 1190-1207; GDR2, 1219-1236. PCR mixtures contained $1 \mathrm{U}$ of BIOTAQ Taq polymerase (Bioline), $1 \times \mathrm{NH}_{4}$ reaction buffer, $1.5 \mathrm{mM} \mathrm{MgCl}, 0.2 \mathrm{mM}$ deoxynucleotide triphosphates (dntps), $0.8 \mu \mathrm{M}$ of forward primer, $0.4 \mu \mathrm{M}$ of each reverse primer and $1 \mu \mathrm{l}$ of undiluted template DNA in a final volume of $25 \mu$ l. Cycling conditions were as follows: initial denaturation at $94{ }^{\circ} \mathrm{C}$ for 5 min, 30 cycles of $94{ }^{\circ} \mathrm{C}$ for $1 \mathrm{~min}, 60^{\circ} \mathrm{C}$ for $1 \mathrm{~min}$, and $72{ }^{\circ} \mathrm{C}$ for $1 \mathrm{~min}$, and a final extension step of $72{ }^{\circ} \mathrm{C}$ for $5 \mathrm{~min}$. PCR products were visualized using a $1.0 \%$ agarose gel stained with ethidium bromide and run in Tris-acetate-EDTA (TAE) buffer at $100 \mathrm{~V}$ for 1 hour. Molecular weight marker X (Roche) was used to estimate sequence length, and amplicons of the correct size were purified using a High Pure PCR Product Purification kit (Roche Applied Science). Purified amplicons were cloned into the 
pCR2.1 vector (Invitrogen) following the manufacturer's instructions, and were inserted into competent DH5 $\alpha$ E. coli cells via heat shock transformation. After overnight growth on LB agar plates containing $200 \mu \mathrm{g} \mathrm{ml}^{-1}$ ampicillin, $100 \mu \mathrm{g} \mathrm{ml} l^{-1}$ isopropyl-beta-Dthiogalactopyranoside (IPTG) and $100 \mu \mathrm{g} \mathrm{m} \mathrm{ml}^{-1}$ 5-bromo-4-chloro-3-indolyl-beta-Dgalactopyranoside (X-Gal), 10 colonies per plate were picked and colony PCR was carried out using M13F (sequence: 5'-CCC AGT CAC GAC GTT GTA AAA CG-3') and M13R (sequence: 5'-AGC GGA TAA CAA TTT CAC ACA GG-3') primers. Colony PCR mixtures contained $0.5 \mathrm{U}$ of BIOTAQ Taq polymerase (Bioline), $1 \mathrm{x}$ bovine serum albumin (BSA) (New England Biolabs), 1 x $\mathrm{NH}_{4}$ reaction buffer, $1.5 \mathrm{mM} \mathrm{MgCl}$, $0.2 \mathrm{mM}$ dntps and $0.4 \mu \mathrm{M}$ of each primer in a final volume of $25 \mu \mathrm{L}$. Cycling conditions were as follows: initial denaturation at $94{ }^{\circ} \mathrm{C}$ for $10 \mathrm{~min}, 30$ cycles of $95{ }^{\circ} \mathrm{C}$ for $1 \mathrm{~min}, 50$ ${ }^{\circ} \mathrm{C}$ for $45 \mathrm{sec}$ and $72{ }^{\circ} \mathrm{C}$ for $2 \mathrm{~min}$, and a final extension step of $72{ }^{\circ} \mathrm{C}$ for $7 \mathrm{~min}$. PCR products were visualized on a $1.0 \%$ agarose gel stained with ethidium bromide and run in TAE buffer at $100 \mathrm{~V}$ for 1 hour. Sequence length was estimated by comparison with molecular weight marker X (Roche).

\section{Sequencing and phylogenetic analysis}

Colony PCR products were digested at $37{ }^{\circ} \mathrm{C}$ overnight using $20 \mathrm{U}$ of $M s p \mathrm{I}$ (New England Biolabs), $1 \times$ reaction buffer and $3.0 \mu \mathrm{L}$ PCR product in a total volume of $10 \mu \mathrm{L}$. Restriction digests were visualized using a 3.0\% agarose gel stained with ethidium bromide and run in $0.5 \mathrm{x}$ TAE buffer at $75 \mathrm{~V}$ for 1.5 hours. Fragment sizes were estimated by comparison with molecular weight marker $\mathrm{X}$ (Roche) and clones were grouped according to restriction fragment length polymorphism (RFLP) banding patterns. 
DNA was quantitated using a Nanodrop 1000 spectrophotometer (Thermo Scientific), and DNA was diluted with sterile, double-distilled water where necessary. Forward and reverse strands of representative clones from each RFLP group were sequenced using a BigDye Terminator v3.1 cycle sequencing kit on an ABI3730 Genetic Analyzer (Applied Biosystems) at the Allan Wilson Centre for Molecular Ecology and Evolution, Massey University.

Vector sequences were removed from the resulting sequences using the online program VecScreen. Forward and reverse sequences were assembled using ContigExpress (Invitrogen), and the presence of chimeric sequences was checked using the online program Bellerophon (Huber et al. 2004). Sequences were compared to those in existing databases using the Basic Local Alignment Search Tool (BLAST) algorithm (Altschul et al. 1990) to identify similar sequences. All sequences were aligned using the Fast Aligner in the ARB software package (Ludwig et al. 2004). Sequences that had $>97 \%$ identity were clustered together using the online program FastGroupII (Yu et al. 2006). Maximum likelihood, maximum parsimony and neighbour-joining trees were obtained using ARB (Ludwig et al. 2004), and consensus trees were constructed based on these initial trees. 


\subsection{Results}

\section{DNA isolation and PCR}

Genomic DNA was successfully isolated from all echinoderm species except Diplopteraster sp. PCR products consistently yielded a single band of the expected size of approximately $900 \mathrm{bp}$, while DNA extraction- and PCR-negative controls did not produce PCR products. Following cloning and RFLP analysis, representative clones were sequenced, producing a total of 156 sequences from all samples (echinoderm tissues, echinoderm surfaces and seawater).

\section{Phylogenetic analysis}

Approximately 50\% of the sequences obtained were isolated from seawater or the surface of the echinoderms only; these sequences were excluded from further analysis. Of the sequences which were obtained from tissue samples, approximately $35 \%$ were also found in seawater or echinoderm surface samples. Sequences that overlapped in this manner, and that were found in tissue samples from less than half of the individuals of each echinoderm species sampled, were also excluded from further analysis, as these were considered to more likely represent contaminating bacteria than SCB. Sequences that were obtained from tissue samples from less than half of the individuals of each echinoderm species, and which were identified as bacteria that clearly differed from previous morphological descriptions of $\mathrm{SCB}$ were also assumed to represent contaminating bacteria and were excluded (see Appendix Figures A1-A10 for phylogenetic trees of echinoderm-associated and seawater bacteria that were excluded as 
potential SCB). Four potential SCB species were thus identified. The first two species, designated SCB species 1 and SCB species 2, were found in only 1 out of 6 samples of their respective hosts, but never on the surface of their hosts or in seawater samples. Species 3 and 4 were found in 4 and 3 out of 6 host samples, respectively, but were also found occasionally in seawater and surface samples (Species 3 was found in 2 out of $6 S$. mollis surface swabs, and 1 out of 5 seawater samples; Species 4 was found in 1 out of 6 surface swabs from Patiriella sp. and S. mollis, as well as in the surface swab from $P$. acuminatus). Figures 2.7 and 2.8 provide more information about the sources of Species 3 and 4.

The first of these species, isolated from S. mollis, falls within the $\alpha$-subdivision of the proteobacteria and is closely related to the SCB previously identified in the brittle star Ophiactis balli (Burnett \& McKenzie 1997), and to members of the order Rhizobiales (Table 2.1). The second species, isolated from S. mollis and Patiriella sp., falls in the $\gamma$ subdivision of the proteobacteria and is closely related to numerous marine invertebrateassociated bacteria (Table 2.2). The symbiotic relatives of this species have been isolated from a wide range of hosts, such as the cortex of a sponge (Thiel et al. 2007b), bivalve gills (Distel et al. 1994) and a vestimentiferan trophosome (Nelson \& Fisher 2000). Species 3, which was found in S. mollis, is an $\alpha$-proteobacterium, belonging to the family Rhodobacteraceae (Table 2.3). Species 4, obtained from S. mollis and Patiriella sp., falls among the $\alpha$ - and $\gamma$-proteobacteria, and is closely related to several pathogenic bacteria such as Legionella spp. and Francisella spp. (Figure 2.8), and more distantly related to 
other $\gamma$-proteobacteria including symbionts of marine molluscs (Durand et al. 1996, Won et al. 2008) (Table 2.4).

Applying the criteria described above, no bacteria from A. scabra, R. mimicus, $P$. acuminatus, P. monocantha or Diplopteraster sp. were identified as likely candidates for SCB. 
Table 2.1. Relationship of SCB species 1 to published sequences.

\begin{tabular}{|c|c|c|}
\hline Organism & Accession no. & Distance \\
\hline Ulva australis surface bacterium & DQ269055 & 0.0192 \\
\hline Hoeflea sp. & EU670237 & 0.0555 \\
\hline Ophiactis balli SCB & U78037 & 0.0580 \\
\hline Watersipora arcuata symbiont & DQ417461 & 0.0580 \\
\hline Bartonella sp. & AB232332 & 0.0581 \\
\hline Alexandrium lusitanicum symbiont & AF359524 & 0.0593 \\
\hline Aminobacter sp. & AY307924 & 0.0606 \\
\hline Defluvibacter lusatiae & AJ132378 & 0.0606 \\
\hline Mesorhizobium sp., sponge-associated & AM183167 & 0.0606 \\
\hline Nitratireductor sp. & AM981316 & 0.0606 \\
\hline Rhizobium leguminosarum & EU329024 & 0.0606 \\
\hline Agrobacterium kieliense & D88524 & 0.0608 \\
\hline Aquamicrobium defluvium & Y15403 & 0.0632 \\
\hline Ochrobactrum sp. & EF028273 & 0.0632 \\
\hline Sinorhizobium medicae & EU271788 & 0.0632 \\
\hline Pseudaminobacter salicylatoxidans & AJ294416 & 0.0644 \\
\hline Thiobacillus sp. & AM403494 & 0.0644 \\
\hline Crabtreella sp. & EU165533 & 0.0657 \\
\hline Mycoplana ramosa & EU022308 & 0.0657 \\
\hline Brucella sp. & DQ305284 & 0.0728 \\
\hline
\end{tabular}

Nearest BLAST matches (one example per genus) are shown, in order of increasing Jukes-Cantor genetic distance from the sequence of SCB species 1 (A value of 0 represents an exact sequence match, and values increase as the evolutionary distance between sequences increases). Only sequences belonging to named species or symbionts of named species were included. 
Table 2.2. Relationship of SCB species 2 to published sequences.

\section{Organism}

Accession no.

AM259846

DQ889927

AM259913

AY321431

AY321389

AJ581351

AY129120

AY129106

L25707

DQ836238

X84979

AF481118

EU377479

AB271123

AY129104

AJ404007

DQ321715

EF103127
0.0331

0.0339

0.0355

0.0404

0.0418

0.0453

0.0741

0.0805

0.0898

0.0921

0.0924

0.0938

0.0949

0.0962

0.0975

0.1003

0.1013

0.1029

Natronocella acetinitrilica

Nearest BLAST matches (one example per genus), in order of increasing Jukes-Cantor genetic distance from the sequence of SCB species 2 (A value of 0 represents an exact sequence match, and values increase as the evolutionary distance between sequences increases). Only sequences belonging to named species or symbionts of named species were included. 
Table 2.3. Relationship of SCB species 3 to published sequences.

\begin{tabular}{|c|c|c|}
\hline Organism & Accession no. & Distance \\
\hline Loktanella marincola & EF202613 & 0.0140 \\
\hline Roseobacter sp. & AF173971 & 0.0140 \\
\hline Ruegeria sp. & AB274753 & 0.0197 \\
\hline Thalassobacter sp. & EU342372 & 0.0269 \\
\hline Octadecabacter orientus & DQ167247 & 0.0283 \\
\hline Sulfitobacter sp. & EU823295 & 0.0283 \\
\hline Oceanibulbus indolifex & EU694388 & 0.0312 \\
\hline Phaeobacter inhibens & AY177712 & 0.0326 \\
\hline Staleya sp. & AJ534233 & 0.0341 \\
\hline Leisingera aquamarina & AM900415 & 0.0370 \\
\hline Thalassococcus halodurans & DQ397336 & 0.0385 \\
\hline Agrobacterium sp. & EU195949 & 0.0399 \\
\hline Methylarcula sp. & AJ534223 & 0.0399 \\
\hline Roseivivax sp. & AB 166990 & 0.0400 \\
\hline Nereida ignava & AJ748748 & 0.0415 \\
\hline Roseisalinus antarcticus & AJ605747 & 0.0415 \\
\hline Oceanicola granulosus & AY424897 & 0.0429 \\
\hline Silicibacter $\mathrm{sp}$. & EF587958 & 0.0429 \\
\hline Citricella sp. & DQ399758 & 0.0458 \\
\hline Nautella italica & AM944522 & 0.0458 \\
\hline
\end{tabular}

Nearest BLAST matches (one example per genus), in order of increasing Jukes-Cantor genetic distance from the sequence of SCB species 3 (A value of 0 represents an exact sequence match, and values increase as the evolutionary distance between sequences increases). Only sequences belonging to named species or symbionts of named species were included. Note that Agrobacterium sp. (Accession no.: EU195949) is probably a misdiagnosis, as all other bacteria listed here belong to the family Rhodobacteraceae. 
Table 2.4. Relationship of SCB species 4 to published sequences.

\begin{tabular}{|c|c|c|}
\hline Organism & Accession no. & Distance \\
\hline Acharax johnsoni symbiont & AJ441188 & 0.0908 \\
\hline Codakia orbicularis symbiont & X84979 & 0.0938 \\
\hline Gigantidas gladius symbiont & EU326224 & 0.0953 \\
\hline Thiohalospira alkaliphila & EU169227 & 0.0968 \\
\hline Curacaobacter baltica & AJ002006 & 0.0998 \\
\hline Oceaniserpentilla haliotidis & AM747817 & 0.0999 \\
\hline Methylobacter alcaliphilus & EF495157 & 0.1043 \\
\hline Pseudomonas stanieri & AB021367 & 0.1043 \\
\hline Thioalkalivibrio versutus & AF126546 & 0.1043 \\
\hline Pseudospirillum japonicum & AB006766 & 0.1043 \\
\hline Halochromatium glycolicum & X93472 & 0.1052 \\
\hline Methylomicrobium sp. & DQ496231 & 0.1058 \\
\hline Oleispira sp. & AY697896 & 0.1060 \\
\hline Colwellia sp. & EF551379 & 0.1073 \\
\hline Shewanella sp. & AB013842 & 0.1073 \\
\hline Thalassomonas loyana & AY643537 & 0.1073 \\
\hline Moritella sp. & AB011363 & 0.1088 \\
\hline Marinomonas mediterranea & AF063027 & 0.1090 \\
\hline Alcanivorax sp. & EU621895 & 0.1121 \\
\hline Vibrio fischeri & EU031644 & 0.1150 \\
\hline
\end{tabular}

Nearest BLAST matches (one example per genus), in order of increasing Jukes-Cantor genetic distance from the sequence of SCB species 4 (A value of 0 represents an exact sequence match, and values increase as the evolutionary distance between sequences increases). Only sequences belonging to named species or symbionts of named species were included. 
The consensus trees displayed in Figures $2.5-2.8$ show the phylogenetic positions of putative SCB species 1-4 respectively. Nearest ARB and BLAST matches are shown, along with the nearest named species. SCB species 1 clearly falls within the $\alpha$ proteobacteria, and is closely related to bacteria belonging to the families Rhizobiaceae and Phyllobacteriaceae in the order Rhizobiales (Figure 2.5). Species 2 falls within the $\gamma$ proteobacteria, in agreement with Table 2.2, and is closely related to symbionts of several other marine invertebrates (Figure 2.6). This group of symbionts appears to belong to the order Chromatiales, otherwise known as the purple sulphur bacteria. Species 3, another $\alpha$ proteobacterium, clearly falls within the family Rhodobacteraceae. There are many representatives of this family present in the marine environment, some of which are shown in Figure 2.7. The final potential SCB identified, Species 4, is a proteobacterium that falls among both $\alpha$ - and $\gamma$-proteobacteria. This species, found in 50\% of Patiriella sp. and S. mollis individuals sampled, is most closely related to several pathogenic bacteria, such as Francisella spp. and Legionella spp., and endosymbionts of insects and paramecia, but is found on a long, naked branch in Figure 2.8. If we examine Table 2.4, we see that the nearest BLAST matches, while not offering quite the resolution provided in Figure 2.8, are much more distant from Species 4 than are the nearest BLAST matches to Species 1-3. These results suggest that Species 4 belongs to an as yet undescribed genus, or perhaps even family. The nearest BLAST match to Species 4 shows $86 \%$ sequence similarity, well below the $>95 \% 16 \mathrm{~S}$ sequence similarity generally accepted as representing bacteria of the same genus (Schloss \& Handelsman 2005). Furthermore, many of the nearest relatives shown in Figure 2.8, which belong to several different 
orders and families, are more closely related to each other than Species 4 is to any of them. 


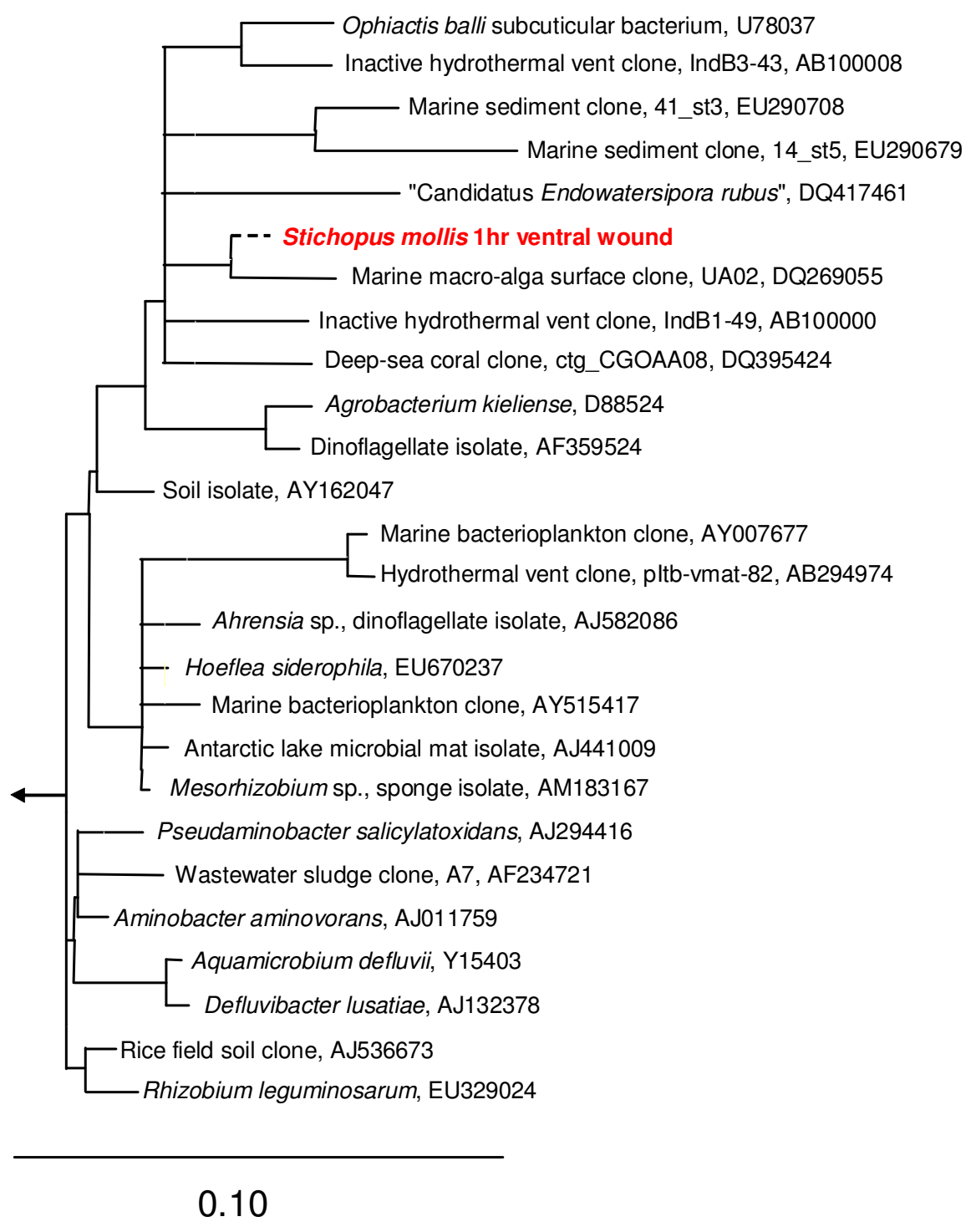

Figure 2.5. 16S rRNA-based phylogeny of putative SCB species 1 (shown in red). The displayed tree is a strict consensus tree based on maximum likelihood, maximum parsimony and neighbour-joining trees, constructed using long ( $>1,000$ nucleotides) sequences. Shorter sequences, belonging to putative SCB, were always added using the parsimony interactive tool in ARB (such sequences are indicated by dashed lines). 


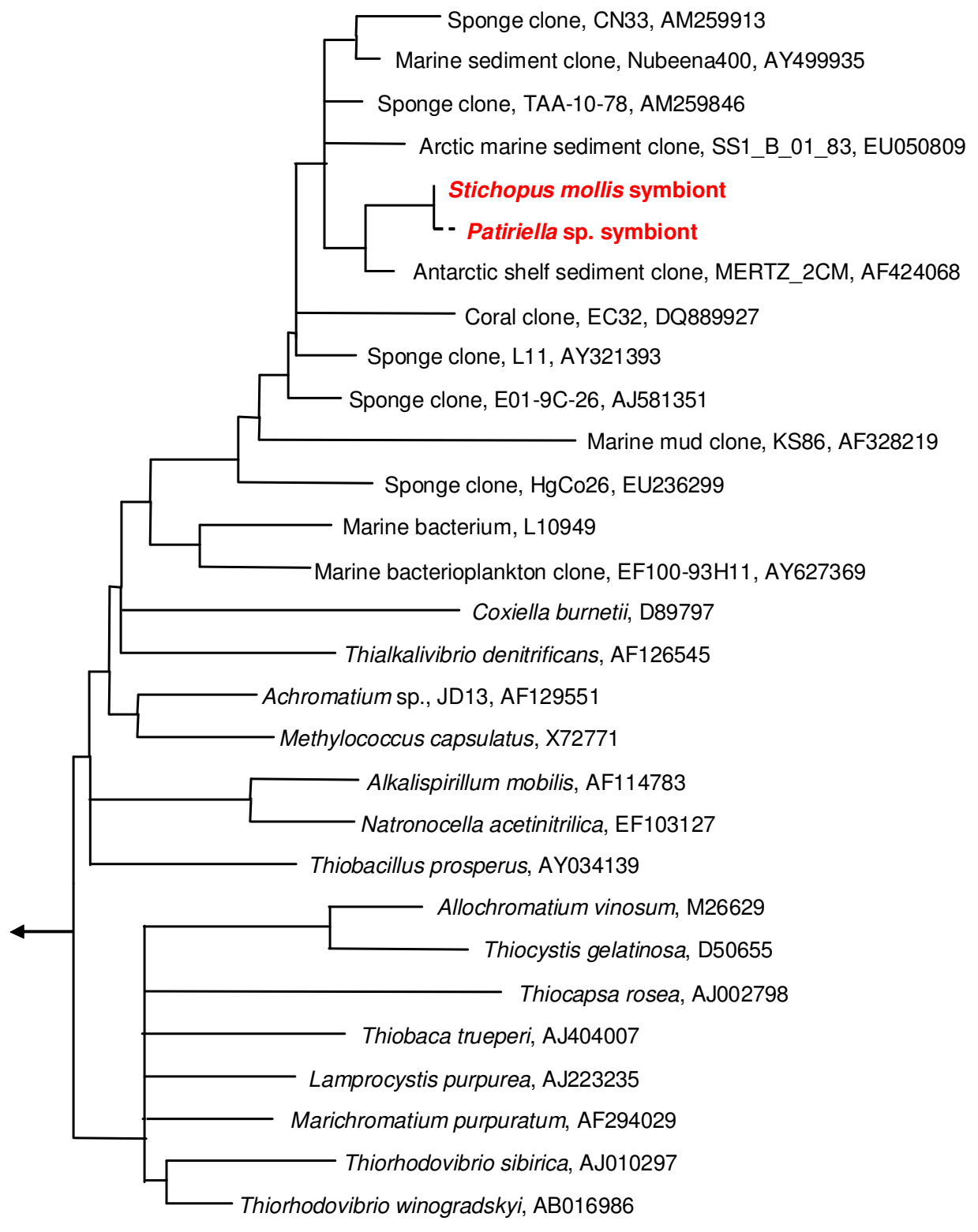

0.10

Figure 2.6. 16S rRNA-based phylogeny of putative SCB species 2 (shown in red). The displayed tree is a strict consensus tree based on maximum likelihood, maximum parsimony and neighbour-joining trees, constructed using long ( $>1,000$ nucleotides) sequences. Shorter sequences, belonging to putative SCB, were always added using the parsimony interactive tool in ARB (such sequences are indicated by dashed lines). Each sequence marked in bold represents a different host individual. 
Chapter 2: Phylogenetic analysis of SCB

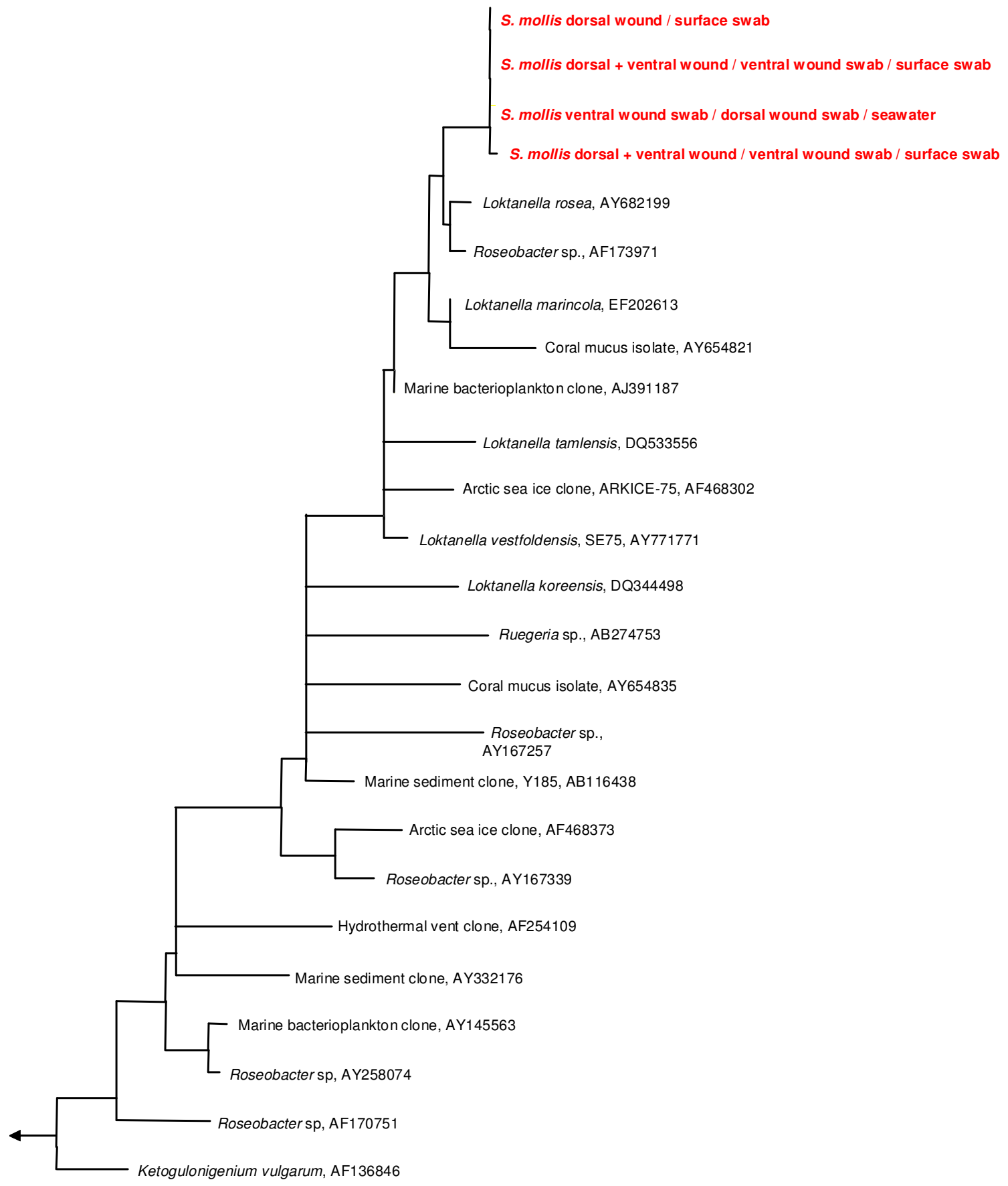

0.10

Figure 2.7. 16S rRNA-based phylogeny of putative SCB species 3 (shown in red). The displayed tree is a strict consensus tree based on maximum likelihood, maximum parsimony and neighbour-joining trees, constructed using long (>1,000 nucleotides) sequences. Shorter sequences, belonging to putative SCB, were always added using the parsimony interactive tool in ARB. Each sequence marked in bold represents a different host individual. 
Chapter 2: Phylogenetic analysis of SCB

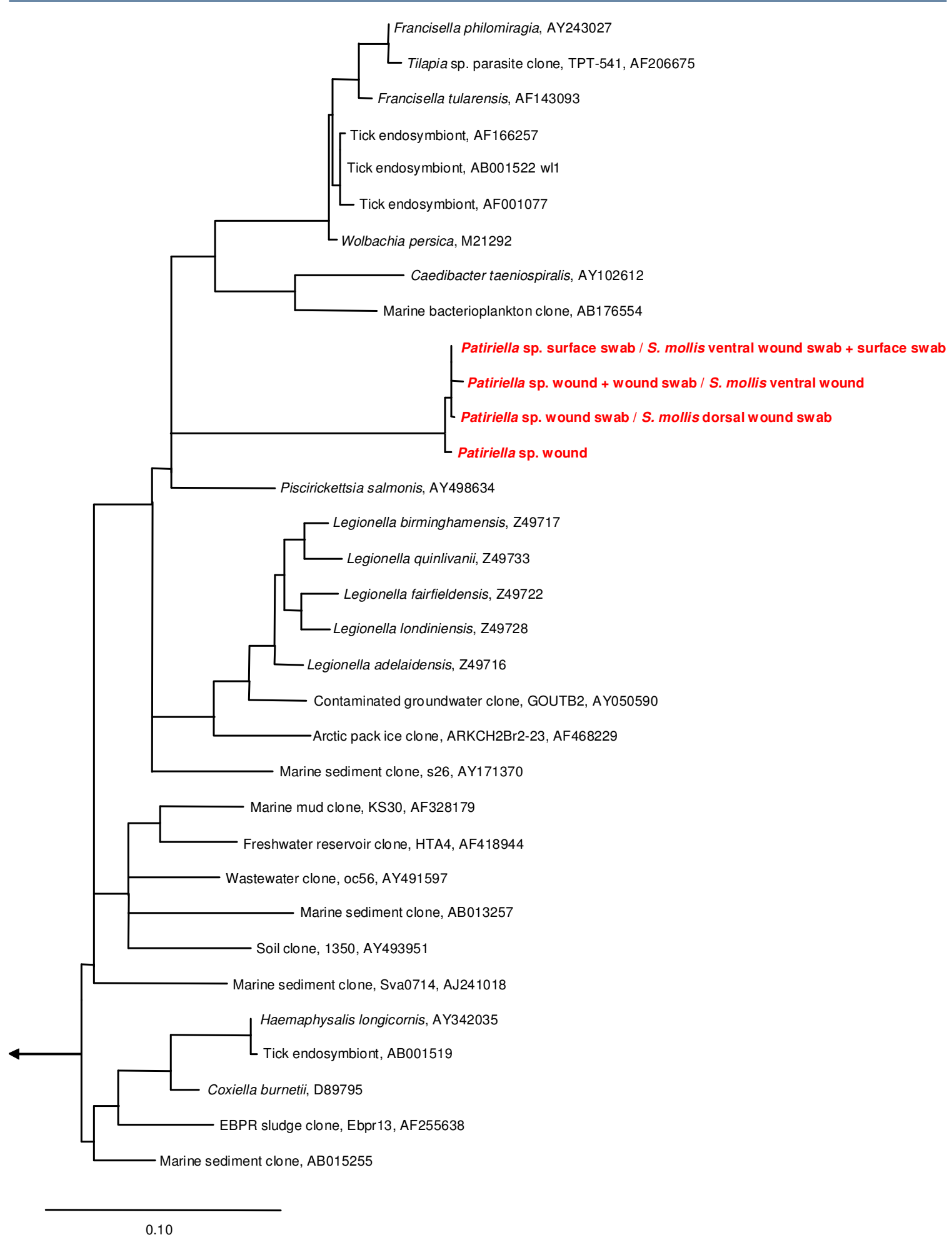

Figure 2.8. 16S rRNA-based phylogeny of putative SCB species 4 (shown in red). The displayed tree is a strict consensus tree based on maximum likelihood, maximum parsimony and neighbour-joining trees, constructed using long (>1,000 nucleotides) sequences. Shorter sequences, belonging to putative SCB, were always added using the parsimony interactive tool in ARB. Each sequence marked in bold represents a different host individual. 


\subsection{Discussion}

\section{Identification of SCB}

Four species of potential SCB were identified in this study. All four species were found in Stichopus mollis, while only 2 of the 4 were found in Patiriella sp. It can be said with some confidence, based on results of the current study and those from previous microscopic work (Kelly et al. 1995) that Astrostole scabra does not contain SCB. The lack of SCB in the deep-water echinoderms studied here (Rosaster mimicus, Psilaster acuminatus, Perissasterias monocantha and Diplopteraster sp.), on the other hand, could be due to inadequate sampling, as only one individual of each species was sampled. Further investigation is needed before ruling out the presence of SCB in these species.

\section{Phylogenetic relationships}

The phylogenetic affiliations of the four bacteria identified as putative SCB are described below. Potential functions of these bacteria in the echinoderm-SCB symbiosis, based on their phylogenetic affiliations, are briefly discussed. A more detailed consideration of the roles these bacteria may be playing in the symbiosis can be found in Chapter 5 .

The four potential SCB identified in the current study fall into quite different groups within the proteobacteria. The first species is closely related to the SCB identified in the brittle star Ophiactis balli (Burnett \& McKenzie 1997). This bacterium falls within the $\alpha$ 2 subdivision of the proteobacteria (Woese 1987), among members of the families Rhizobiaceae and Phyllobacteriaceae in the order Rhizobiales. Members of these families 
include pathogens and symbionts of plants and animals. Examples of pathogens in these families include Brucella spp., the causative agents of brucellosis in animals and humans (Whatmore et al. 2008), and Agrobacterium spp., which cause tumours in plants (DafnyYelin et al. 2008). Perhaps the best known symbionts among this group are the nitrogen fixing Rhizobia, comprised of the genera Rhizobium, Sinorhizobium, Mesorhizobium and Phyllobacterium (Kan et al. 2007). A bacterium of the genus Mesorhizobium has also recently been found in association with the marine sponge Phakiella ventilabrum (Krick et al. 2007). The close relationship of Species 1 to nitrogen-fixing Rhizobia may suggest that it is involved in nitrogen fixation itself. This is not an unreasonable explanation, given that symbiotic bacteria of echinoderms (Guerinot \& Patriquin 1981) and other marine invertebrates (Carpenter \& Culliney 1975, Kneip et al. 2007, Weisz et al. 2007) have been shown to fix nitrogen and transfer this to their hosts.

Species 2 falls within the $\gamma$-proteobacteria, clustering with several other marine symbionts. The closest published sequence to this putative SCB belongs to a spongeassociated bacterium (Thiel et al. 2007a). The group in which the second SCB species falls appears to belong to the order Chromatiales. The bacteria within this order exhibit a wide range of characters, with some members being anaerobic phototrophs (Rees et al. 2002), others microaerophilic lithoautotrophs (Sorokin et al. 2002), and others still aerobic chemoorganoheterotrophs (Lee et al. 2007). Generally speaking, however, bacteria in this order are sulphur-oxidizing and autotrophic (Pfennig \& Trüper 1989). As with Species 1, it is possible that Species 2 is involved in nitrogen fixation, as some members of the order Chromatiales are known to be nitrogen-fixing bacteria (Pfennig \& 
Trüper 1989). Alternatively, given that most Chromatiales bacteria are autotrophic, this bacterium may provide an energetic benefit to the host, through translocation of chemosynthate. Finally, as the order Chromatiales consists of sulphur-oxidizing bacteria, a role in detoxification of hydrogen sulphide can not be ruled out. Hydrogen sulphide is toxic to animals, and bacterial symbionts of marine invertebrates have been shown to carry out detoxification (Vetter 1985, Giere \& Langheld 1987, Temara et al. 1993).

Species 3 is an $\alpha$-proteobacterium belonging to the family Rhodobacteraceae. Members of this family are commonly found in the marine environment (Brinkhoff et al. 2008), and several are symbionts of invertebrates, including marine sponges (Althoff et al. 1998) and terrestrial isopods (Wang et al. 2007). The nearest relatives of Species 3, shown in Figure 2.7, belong to the genera Roseobacter, Loktanella, Ruegeria and Ketogulonicigenium. These four genera belong to the Roseobacter clade. Members of this clade are found in a wide variety of marine habitats, and have a wide range of physiological characteristics and trophic habits (Brinkhoff et al. 2008); some are heterotrophic (Gonzalez et al. 2000), while others are autotrophic (Brinkhoff et al. 2008), but all are aerobic and aquatic (Brinkhoff et al. 2008). Some members of this clade, such as Roseobacter spp. and Ruegeria spp., form symbioses with dinoflagellates (Brinkmeyer et al. 2000, Groben et al. 2000, Amaro et al. 2005), and there is evidence that they may be responsible for inducing toxin production in certain dinoflagellates through production of secondary metabolites (Silva 1990, Prokic et al. 1998). There are numerous examples of members of this clade forming associations with marine invertebrates and algae (WagnerDöbler \& Biebl 2006), although in several cases the relationship is pathogenic rather than 
symbiotic (e.g. in corals (Cooney et al. 2002, Pantos et al. 2003) and bivalve molluscs (Boettcher et al. 2000, Maloy et al. 2007)). Despite the wide variety of characteristics possessed by members of the Roseobacter clade, there are some traits common to most members. One such trait is the production of secondary metabolites (Buchan et al. 2005). Some members of the genus Roseobacter are known to produce secondary metabolites with antimicrobial activity (Brinkhoff et al. 2004, Hjelm et al. 2004), and if Species 3 produces similar compounds, it may be responsible for the hypothesized antimicrobial function of SCB (Dobson 2001, Strahl et al. 2002). Another possibility, however, is that Species 3 is a pathogen of its host, S. mollis. Pathogenic Roseobacter species are known from marine invertebrates, and may be associated with skin ulceration disease in the sea cucumber Holothuria scabra (Becker et al. 2004).

The fourth species identified here falls among the $\alpha$ - and $\gamma$-proteobacteria, and its near relatives consist mainly of pathogenic bacteria and symbionts of insects. The nearest named species to Species 4 belong to the genera Piscirickettsia, Francisella, Caedibacter and Wolbachia. The former two genera belong to the order Thiotrichales and the latter two belong to the order Rickettsiales. Of these four genera, only Caedibacter consists of truly mutualistic or commensal bacteria, which are endosymbionts of Paramecium spp. (Beier et al. 2002). The three other genera mentioned above are all predominantly parasitic or pathogenic, with Piscirickettsia spp. infecting fish (Fryer et al. 1992, House et al. 2006), Francisella spp. infecting numerous animals including humans (Keim et al. 2007), and Wolbachia spp. infecting insects (Hertig 1936) and nematodes (Rao 2004). (Note that there are examples of Wolbachia forming mutualisms with insects (Dedeine et 
al. 2001) and nematodes (Bandi et al. 1999), but these are far less common than parasitic Wolbachia symbioses). SCB Species 4 does appear to belong to a different genus, and perhaps family, from these genera, based on the high level of sequence divergence between these genera and Species 4 . Nevertheless, it is interesting that so many of its nearest relatives are pathogenic or parasitic in nature. Any function that Species 4 may have in the echinoderm-SCB symbiosis is not immediately evident, as there are no reports of bacteria in the orders Rickettsiales of Thiotrichales forming non-parasitic symbioses with marine animals.

\section{Limitations of methods}

The true diversity of the SCB community in the echinoderm species studied here may have been underestimated for two reasons. The first is the exclusion of sequences which were found in seawater and/or echinoderm surface samples, but also occasionally in tissue samples (Tissue sample-derived sequences which were found in less than half of the individuals of each echinoderm species sampled were excluded if also found in seawater or on the echinoderm surface). As little is known of the mode of transmission of SCB (i.e. vertical transmission from parent to offspring or horizontal transmission via uptake from seawater), it is entirely possible that bacteria which were identified on the echinoderm surface or in seawater as well as in tissue samples are in fact symbiotic. While little evidence exists, there has been some suggestion that vertical transmission is the more likely scenario (McKenzie et al. 1998), as the distribution of different morphological groups of SCB is tightly linked with host systematics (Kelly et al. 1995, Kelly \& McKenzie 1995). Indeed, SCB have been identified in the larvae of the sea star 
Luidia sp. (Class Asteroidea) (Bosch 1992) and in the brooded embryos of the brittle star Amphipholis squamata (Class Ophiuroidea) (Walker \& Lesser 1989), suggesting vertical transmission. Vertical transmission has also been demonstrated in other marine symbioses, such as those between the sponge Ircinia felix and its microbial inhabitants (Schmitt et al. 2007), and the bivalve Solemya velum and its microbial symbionts (Krueger et al. 1996). On the other hand, uptake of bacterial symbionts from surrounding seawater has also been demonstrated in several marine symbioses, examples being the squid Euprymna scolopes and its luminous Vibrio fischeri symbionts (Ruby 1996, Nyholm et al. 2000), and the tubeworm Riftia pachyptila and its bacterial symbionts (Cary et al. 1993). Moreover, Barker and Kelly (Barker \& Kelly 1994) were unable to demonstrate symbiont transmission from parent to offspring in three SCB-containing echinoids in laboratory experiments. Whether vertical or horizontal transmission occurs in the echinoderm-SCB symbioses studied here, there is certainly the potential for seawater-borne bacteria to also be SCB and the true diversity may thus have been underestimated. It was considered preferable, however, to act conservatively and risk underestimating the true number of species rather than include all sequences found in tissue samples and risk labeling seawater or surface-associated contaminants as SCB.

The second way in which the true diversity may have been underestimated was the use of proteobacteria-specific PCR primers to amplify the DNA of the bacteria. While it would have been preferable to use universal or eubacteria-specific primers in order to identify a wider potential range of bacteria, this was not feasible due to specificity and contamination issues (see appendix). However, the choice of primers likely did not affect 
the ability to detect SCB to a great degree for several reasons. First, preliminary work using the eubacteria-specific primer pair 27f / 1492r (prior to contamination issues arising) found almost entirely proteobacteria in tissue, surface and seawater samples (data not shown). Furthermore, previous studies have identified SCB of other echinoderm species as $\alpha$-proteobacteria (Burnett \& McKenzie 1997) and $\gamma$-proteobacteria (Lesser \& Blakemore 1990). Second, while not amplifying certain bacterial groups that the 27f / 1492r primer pair does, the overall number of species amplified by the proteobacteria primers is in fact higher. Using the Probematch function of the Ribosomal Database Project (Maidak et al. 2001), and searching against only good quality sequences at least $1200 \mathrm{bp}$ in length, it was found that the $27 \mathrm{f} / 1492 \mathrm{r}$ primer pair matches at most $\sim 8 \%$ of all sequences. The proteobacteria primers used in this study, on the other hand, match $37 \%$ of sequences in the database. Although the vast majority of this figure is made up of proteobacteria, it still represents a significant increase in coverage, and balances out the loss of coverage in other bacterial groups. Finally, the loss of coverage in groups outside the proteobacteria mentioned above is further negated by the fact that a substantial proportion of those bacteria which match the eubacterial primers but not the proteobacteria primers are in the Gram-positive phyla such as Firmicutes and Actinobacteria. Approximately $20 \%$ of sequences matched by the $27 \mathrm{f} / 1492 \mathrm{r}$ primer pair are Gram-positives, whereas approximately $12 \%$ of matches to the proteobacteria primer set are Gram-positives. Previous work has shown that SCB are typically Gram-negative, based on morphology (Holland \& Nealson 1978, McKenzie \& Kelly 1994, Kelly et al. 1995), cultivation requirements (Lesser \& Blakemore 1990) and phylogenetic identification (Burnett \& McKenzie 1997). 
Just as the true SCB diversity may have been underestimated, the possibility exists that the four bacteria identified here might not all be SCB. As Species 1 and 2 were only found in 1 out of 6 samples of their respective hosts, the possibility exists that they in fact originated from a source other than the sub-cuticular space of the host. Previous studies have found that different individuals of the same echinoderm species will all contain SCB, if indeed the echinoderm is an SCB-containing species (Kelly et al. 1994, Kelly et al. 1995). This would suggest that if Species 1 and 2 are in fact SCB, they should be found in all of the individuals sampled. There are at least two factors which need to be taken into account before ruling these species out as SCB however.

First, it has been noted that SCB abundance can vary across time and space (Foret and Lawrence (2001) found that half of the echinoderms they studied had significantly lower SCB densities in winter than in autumn, and that SCB densities in the ophiuroid Ophiophragmus filograneus varied significantly among different populations), as well as in response to stress (Newton \& McKenzie 1995). It is therefore possible that Species 1 and 2, while present in all individuals, were only present in high enough numbers to be detected in 1 individual of each host species. While PCR is in theory sufficiently sensitive to amplify DNA from as little as one cell, in practice the more abundant bacteria are preferentially amplified, and rarer bacteria my be missed.

The second factor which needs to be considered is that SCB community composition may vary between individuals of the same echinoderm species, resulting in detection of a 
particular SCB species in only a minority of individuals. Previous workers have found that if an echinoderm species contains SCB, then all individuals of that species will contain SCB (McKenzie \& Kelly 1994, Kelly et al. 1995). However, these studies have all relied on microscopy, and therefore it is not known whether different individuals of the same echinoderm species contain the same species of bacteria in their SCB communities. Only one study to date has identified SCB phylogenetically, and this was in one species of echinoderm only (Ophiactis balli, an ophiuroid) (Burnett \& McKenzie 1997). There appear to be three potential scenarios with regards to the SCB community composition of conspecific echinoderms: a) All conspecific, SCB-containing echinoderms contain the same few SCB species, b) Different individuals contain one or more core SCB species, which are common to all individuals, along with one or more SCB species which are variable among individuals, c) Conspecific individuals, while all containing SCB (perhaps through some morphological adaptation that allows them to take up and contain the bacteria), each contain completely different bacteria (a scenario which would presumably imply horizontal transmission of SCB randomly from the seawater). While scenario $\mathrm{C}$ is unlikely in the species studied here, as SCB Species 3 and 4 were found in at least $50 \%$ of their host echinoderms, Scenario B cannot be ruled out. Therefore, while it remains impossible to prove conclusively from the results presented here, we cannot rule out the possibility that Species 1 and 2 are SCB.

Species 3 and 4 cannot be definitely identified as SCB for other reasons. These two species were both found in at least half of the individuals of their respective host species (Species 3 was found in 4 out of 6 S. mollis individuals, Species 4 was found in 3 out of 6 
Patiriella sp. and S. mollis individuals). DNA extraction techniques can miss some rare bacteria, and PCR tends to preferentially amplify more abundant bacteria (Theron \& Cloete 2000). Thus, if the abundance of Species 3 and 4 varies among individuals as suggested in the previous paragraph for Species 1 and 2, it is possible that Species 3 and 4 are in fact present in all of the individuals sampled, but failed to show up in PCRs from some of the samples. If so, this would suggest that these two species are SCB. However, Species 3 was also found in seawater and echinoderm surface samples, and Species 4 was found in surface samples as well. This raises the possibility that both of these species are in fact contaminants from the host surface or surrounding seawater (as mentioned earlier, this is the reason many of the sequences obtained in this study were excluded as potential SCB). Although it is not possible to discount this possibility entirely, it is considered unlikely that these two species merely represent contamination. Seawater and surface samples were taken along with each set of tissue samples, and were subjected to identical DNA extraction and PCR protocols. Surface swabs were taken in exactly the same manner as wound swabs, and although the seawater sampling method was rather different to those used for tissue and surface samples, bacterial DNA was consistently extracted. Despite this, Species 3 and 4 were only rarely found in seawater or surface samples (Species 3 was obtained from 1 out of 6 surface swabs from Patiriella sp. and S. mollis; Species 4 was obtained from 2 out of 6 S. mollis surface swabs and 1 out of $51 \mu \mathrm{m}$ filtered seawater samples), yet were isolated from tissue samples in at least half of the individuals in which they were found. It therefore appears that Species 3 and 4 were much more abundant in the tissue samples than on the surface of the echinoderms and in the surrounding seawater. The presence of these two bacteria on the surface of the 
echinoderms or in seawater could provide evidence for horizontal transmission, whereby the echinoderms obtain their SCB from seawater. However, as the seawater in which the echinoderms were stored had been pre-filtered, another possibility should be considered that the presence of Species 3 and 4 in the seawater was due to the presence of the echinoderms in the water. Seawater samples were taken after leaving the echinoderms in the seawater overnight. Therefore, it is possible that Species 3 and 4 were actually transferred from echinoderm to seawater, rather than vice versa. Either scenario could easily account for the occasional presence of these bacteria in seawater and echinoderm surface samples. All things considered, it seems more probable that Species 3 and 4 are SCB rather than contaminating, seawater-borne bacteria.

\section{Concluding remarks}

In conclusion, this study has found that SCB are likely present in at least two of the species examined, Patiriella sp. and S. mollis, and that in both echinoderm species, there may be more than one species of SCB present. Four species of putative SCB were identified, all of which were found in tissue samples from S. mollis, and two of which were found in tissue samples from Patiriella sp. The first putative SCB species, identified in S. mollis, is closely related to the previously published sequence of the SCB from an ophiuroid (Burnett \& McKenzie 1997), while the second is closely related to bacteria associated with several other marine invertebrates. The phylogenetic positions of these two species allow us to make some suggestions about their potential functions in the symbiosis, and it appears likely that they are playing a nutritional role, although the exact nature of this role has yet to be determined. The third and fourth putative SCB species are 
less closely related to truly symbiotic bacteria than Species 1 and 2, and it is therefore more difficult to determine what role they may be playing in the echinoderm-SCB symbiosis. It is possible that they are involved in production of compounds which confer antimicrobial protection on the host, or they may simply be pathogens or parasites. Clearly there are still many questions to be answered. In Chapter 3, the phylogenetic work conducted here is expanded upon through the use of fluorescence in situ hybridization (FISH), in an attempt to better understand the composition of the SCB community in several echinoderms; and in Chapter 4 attempts are made to culture SCB. These are only the first steps towards fully understanding the nature of the echinodermSCB symbiosis, and further work is needed to determine potential nutritional or energetic benefits to host and bacteria, the mode of transmission of the symbionts, and whether or not the same or similar bacteria are found across a wider range of host species. The latter objective can be achieved through the use of methods similar to those used here, albeit on a much larger scale, and the estimation of a reliable phylogeny of the host species used would be a useful tool for determining if co-evolution is occurring between host and symbiont. The first two objectives may be somewhat more difficult to achieve. Gene expression assays may help to elucidate the function of $\mathrm{SCB}$, and the mode of transmission may be better understood through transfection experiments similar those carried out by Barker and Kelly (1994), although their attempts at transferring SCB from parent to offspring were unsuccessful. A better understanding of SCB in general is hampered by the difficulty faced in attempting to culture these bacteria, although these attempts are ongoing (N. Dayasam, personal communication). Whichever way is chosen 
to move forward, it is evident that there is still a lot to be learned about this intriguing symbiosis. 


\title{
Chapter 3: Fluorescence in situ hybridization analysis of sub- cuticular bacteria
}

\begin{abstract}
Sub-cuticular bacteria (SCB) are common endosymbionts of a wide range of echinoderms. These bacteria, which appear to be gram-negative bacilli, have yet to be identified. Likewise, the role they play in the symbiosis is unknown. In the present study, fluorescence in situ hybridization (FISH) was used to determine the phylogenetic affiliations of SCB in two common New Zealand echinoderms, Patiriella sp. (class Asteroidea) and Stichopus mollis (class Holothuroidea). Through the use of a range of FISH probes, varying in specificity from domain to species, it was found that the SCB community of Patiriella sp. is made up almost entirely ( $85 \%)$ of bacteria belonging to the $\alpha$-subdivision of the proteobacteria. The SCB community of $S$. mollis contained a similar proportion of $\alpha$-proteobacteria $(\sim 85 \%)$, but differed from Patiriella $\mathrm{sp}$. in that it also contained a small percentage of $\gamma$-proteobacteria. Observations of SCB morphology revealed that the $\alpha$-proteobacterial symbionts of both species are curved or spiral-shaped rods, $\sim 1-2 \mu \mathrm{m}$ in length, which are similar to the commonly observed 'Type 2' SCB which have been previously described from a range of echinoderms. The $\gamma$-proteobacteria seen here are slightly smaller rod-shaped bacteria which may also be Type 2 SCB. The SCB of these species could not be identified to species level, but this is still the first study to demonstrate the phylogenetic affiliations of SCB in situ.
\end{abstract}




\subsection{Introduction}

Bacterial symbionts living in the sub-cuticular space of echinoderms were originally reported in 1978 (Holland \& Nealson 1978). This initial report found these sub-cuticular bacteria (SCB) in echinoderms belonging to four of the five extant echinoderm classes (Asteroidea, Crinoidea, Echinoidea and Ophiuroidea), and shortly thereafter they were also discovered in members of the class Holothuroidea (Feral 1980). In the years since these initial discoveries, it has been found that SCB are in fact very common, with more than half of all echinoderm species studied so far containing them (Kelly et al. 1994, Kelly et al. 1995, Kelly \& McKenzie 1995).

Unlike many other invertebrate-microbe symbioses which have received considerable attention (for examples, see reviews of microbial interactions with corals (Rosenberg et al. 2007) and sponges (Taylor et al. 2007)), little is known about the echinoderm-SCB symbiosis. What is known about $\mathrm{SCB}$, aside from their aforementioned commonness, is that they are gram-negative, rod-shaped bacteria that can be divided into three types based on their morphology (McKenzie \& Kelly 1994, Kelly \& McKenzie 1995). Type 1 SCB are small rods, approximately $1 \mu \mathrm{m}$ in length, which are made up of two bacteria sharing the same outer capsule. Type 2 SCB consist of thin rods, generally 1-2 $\mu \mathrm{m}$ in length, which vary from straight to spiral-shaped and which have little internal specialization. Type $3 \mathrm{SCB}$ are similar in length to Type $2 \mathrm{SCB}$, but are generally thicker and contain membrane-bound vacuoles. 
Almost all studies of the echinoderm-SCB symbiosis so far have relied on microscopy, which has been useful in terms of describing the morphology, location and abundance of the bacteria, but has limited usefulness in determining their identity or function. Attempts to assign a function to SCB have, for the most part, been somewhat speculative, relying on microscopic observations such as the particular locations of SCB within the host. For example, it has been suggested that SCB provide the host with energy, as partially digested SCB have been observed inside phagosomes of host epidermal cells in several holothurians (Roberts et al. 1991) and in larvae of the asteroid Luidia sp. (Bosch 1992). It is not known, however, if the hosts actively feed on SCB or whether they only phagocytose moribund SCB, and whether or not the energy or nutrients gained make up a substantial proportion of the hosts' diets. Likewise, the rapid colonization of ophiuroid wound sites by SCB has led to the suggestion that SCB prevent infection by pathogenic bacteria through competitive exclusion or production of an antimicrobial compound (McKenzie \& Kelly 1994, Strahl et al. 2002). There is, however, no empirical proof that SCB do act in this way; Strahl et al. (2002) have shown that bacteria isolated from the ophiuroid Amphipholis squamata show antimicrobial action, but they could not definitively say whether the isolated bacteria were SCB. These examples are not presented with the intention of refuting the authors' hypotheses, but rather as an illustration of the limitations imposed by the reliance on conventional techniques such as electron microscopy. Clearly, other approaches are needed if we are to determine the function and identity of SCB. Empirical studies have been carried out to determine whether SCB contribute to host energy or nutrition, and while it has been shown that in some ophiuroids the SCB do indeed translocate amino acids from seawater to host, it has 
not yet been proven if this provides a substantial benefit to the host (Walker \& Lesser 1989, Lesser \& Walker 1992).

Studies such as those carried out by Strahl et al. (2002), looking at antimicrobial activity of SCB, and Lesser and Walker (1992), examining amino acid uptake by SCB, are obviously very important in terms of determining function. In addition to such studies, however, it would be desirable to see more use of molecular techniques, such as the polymerase chain reaction (PCR), DNA sequencing, and fluorescence in situ hybridization (FISH), which may enable us to establish the identity of SCB. By finding out the phylogenetic affiliations of SCB, we may also gain a better understanding of their function. The only work done so far on SCB using molecular techniques is that of Burnett and McKenzie (1997), who identified the SCB of the ophiuroid Ophiactis balli by sequencing the $16 \mathrm{~S}$ rRNA gene of the symbiont. It was found that the $O$. balli symbiont belonged to the $\alpha$-proteobacteria, and was closely related to the nitrogen-fixing agrobacteria, a finding which may explain the role that these bacteria play in the symbiosis. Obviously this is still not conclusive evidence for the function of SCB, but such studies, combined with conventional methods such as microscopy and cultivation, offer the most hope of determining the function of the echinoderm-SCB symbiosis.

In the present study, the use of molecular techniques to study SCB was expanded upon through the use of FISH. Fluorescence in situ hybridization involves the binding of fluorescently-labeled oligonucleotide probes to a specific region of rRNA in target bacteria. The fluorescently-labeled probe can then be visualized using an epifluorescence 
or confocal microscope, and the targeted bacteria can thus be identified. By using probes of varying specificity, bacteria can be identified at different taxonomic levels, from domain through to species. As the name suggests, the technique is carried out in situ (in this study, in sections of echinoderm tissue), and this results in the ability to determine where exactly in a given sample the bacteria are located. The present study used FISH probes targeting eubacteria, proteobacteria, and two bacterial species previously identified as putative SCB (see Chapter 2), to identify the SCB in the echinoderm species Patiriella sp., Stichopus mollis, and Astrostole scabra. Observations were also made of the morphology of the SCB present, and the proportions of the SCB communities composed of particular bacterial taxa.

\subsection{Materials and methods}

\section{Sample collection}

The three species of echinoderm used in this study were Patiriella sp. (class Asteroidea), Stichopus mollis (class Holothuroidea) and Astrostole scabra (class Asteroidea). The former two species are known to contain SCB, while A. scabra does not contain SCB (Kelly et al. 1994) (See also Chapter 2), and was therefore used as a negative control for FISH experiments. Two individuals of each species were collected from Island Bay, Wellington, New Zealand (latitude $41^{\circ} 20^{\prime} \mathrm{S}$, longitude $174^{\circ} 46^{\prime} \mathrm{E}$ ) in February 2008, and transferred directly to plastic buckets containing seawater. Echinoderms were rinsed with $1 \mu \mathrm{m}$-filtered seawater (FSW) within two hours of collection and left in FSW overnight. 


\section{Sample fixation and decalcification}

Following overnight storage in FSW, echinoderms were rinsed for 30 seconds in sterile artificial seawater (ASW), and tissue samples, approximately $2 \mathrm{~cm}^{2}$ in size, were excised from each of the echinoderms using a sterile scalpel. For Patiriella sp. and A. scabra, this consisted of the removal of arm tips, and for S. mollis both ends of the animal were removed for fixation. All tissue samples were fixed in $50 \mathrm{ml}$ volumes of $3.7 \%$ formaldehyde in 1 x phosphate-buffered saline (PBS), mixed on a laboratory rotator at 4 ${ }^{\circ} \mathrm{C}$ for 12 hours. Following fixation, samples were rinsed 3 times in $1 \mathrm{x}$ PBS, and decalcified. Decalcification was necessary as the skeleton and spines of echinoderms are made of calcium carbonate, and it was necessary to remove as much of this as possible in order to soften the tissue for subsequent sectioning. The decalcification procedure was as follows. Fixed samples were placed in polypropylene tubes containing $50 \mathrm{ml} 0.5 \mathrm{M}$ ethylenediaminetetraacetic acid (EDTA). Tubes were rotated at $4{ }^{\circ} \mathrm{C}$, and every 24 hours the liquid was removed and fresh EDTA was added. After 96 hours, decalcification was stopped. Ninety-six hours of decalcification was found to soften the tissue sufficiently without adversely affecting the tissue morphology (additional tissue samples were decalcified for $24,48,72$ and 120 hours, and were found to either be too calcified for sectioning or have lost their original morphology).

\section{Tissue processing, embedding and sectioning}

Tissues were dehydrated using an ethanol series, and cleared using xylene, according to the following regime: 45 minutes in $30 \%$ ethanol, 45 minutes in $40 \%$ ethanol, 45 minutes in 50\% ethanol, 45 minutes in $60 \%$ ethanol, 45 minutes in $70 \%$ ethanol, 45 minutes in 
$80 \%$ ethanol, 45 minutes in $95 \%$ ethanol, 45 minutes in $100 \%$ ethanol, 60 minutes in $100 \%$ ethanol, 60 minutes in $100 \%$ ethanol / 100\% xylene (1:1), 60 minutes in 100\% xylene, 60 minutes in $100 \%$ xylene. All steps were performed in $50 \mathrm{ml}$ polypropylene tubes, rotated at $4{ }^{\circ} \mathrm{C}$. Following dehydration and clearing, tissues were embedded in paraffin wax. Embedding consisted of placing the tissue samples into molten Paraplast Xtra Tissue Embedding Medium (McCormick Scientific), incubating at $50{ }^{\circ} \mathrm{C}$ for 60 minutes, and repeating the process twice. Following these embedding steps, blocks of Paraplast X-tra were poured, tissue samples were inserted, and the blocks were left overnight at $4{ }^{\circ} \mathrm{C}$ to set. The following day, the paraffin blocks were removed from their casts, trimmed to size and mounted on wooden blocks.

Sectioning was carried out using an American Optical 820 rotary microtome. Transverse sections were cut at $8 \mu \mathrm{m}$ thickness, as thinner sections proved difficult to cut and did not provide any advantage in terms of FISH probe-binding efficiency or microscopy (data not shown). Tissue sections were floated in a $40{ }^{\circ} \mathrm{C}$ water bath and then transferred to Superfrost Plus slides (Esco).

\section{FISH}

Prior to performing hybridizations, slides were deparaffinized as follows: 3 minutes in $100 \%$ xylene, 3 minutes in $100 \%$ xylene, 3 minutes in $100 \%$ xylene / 100\% ethanol (1:1), 3 minutes in $100 \%$ ethanol, 3 minutes in $100 \%$ ethanol. All steps were carried out in glass Coplin jars in a laminar flow hood. Slides were then left to dry in the laminar flow hood. 
Hybridizations were carried out as follows: Circular wells, approximately $2 \mathrm{~cm}$ in diameter, were drawn around tissue sections on slides using an Aquahold pap pen (Scientific Device Laboratory). Into these wells were placed $80 \mu$ hybridization buffer (see Appendix Table A2 for hybridization buffer formula), and $8 \mu$ of FISH probe (50 ng $\mu l^{-1}$ ). When dual hybridizations were performed (i.e. two probes in the same hybridization), the amount of hybridization buffer was increased to $160 \mu \mathrm{l}$, and $8 \mu \mathrm{l}$ of each probe was used. Once slides had been prepared as above, each was placed into a 50 $\mathrm{ml}$ polypropylene screw-top tube, along with a piece of laboratory tissue soaked with approximately $1.5 \mathrm{ml}$ of hybridization buffer. Tubes were screwed tightly shut and placed in a hybridization oven (Agilent Technologies) at $46{ }^{\circ} \mathrm{C}$. After 2 hours, slides were removed from tubes, washed briefly with wash buffer (See Appendix Table A3 for wash buffer formula), and placed into $50 \mathrm{ml}$ polypropylene tubes containing $50 \mathrm{ml}$ wash buffer pre-warmed to $48{ }^{\circ} \mathrm{C}$. Tubes were incubated at $48{ }^{\circ} \mathrm{C}$ for 15 minutes in a water bath, then removed and washed in sterile double-distilled water. An important consideration in terms of hybridization stringency is the formamide concentration used in the hybridization buffer (See next section). When hybridizations were performed using two probes which required different formamide concentrations, the probe requiring the higher concentration was hybridized and washed first, as described above, then the second probe was hybridized at the lower concentration and washed. After washing, water droplets were removed from tissue sections by directing compressed air at the side of slides, and slides were left in the dark to dry completely. Once dry, wells were removed with xylene and coverslips were mounted using Prolong Gold antifade mounting medium (Invitrogen), with or without the DNA stain 4',6-diamidino-2-phenylindole (DAPI) (DEPEX was also 
trialed as a mounting medium, but Prolong Gold was found to provide better antifade properties). Slides were left for 24 hours in order for the mounting medium to cure before viewing using an Olympus Fluoview FV1000 confocal laser scanning microscope (CLSM).

\section{Probe choice and design}

A range of previously published probes were used in the present study, along with two newly designed probes. Probes were fluorescently labeled by addition of a cyanine dye at the 5' end, and were purchased from Thermo Fisher Scientific, Germany. Table 3.1 provides information on each of the probes used. 
Table 3.1. FISH probes used in this study.

\begin{tabular}{|c|c|c|c|c|}
\hline $\begin{array}{c}\text { Probe } \\
\text { name and } \\
\text { source }\end{array}$ & $\begin{array}{c}\text { Probe sequence (5'-3') } \\
\text { Fluorescent label }\end{array}$ & $\begin{array}{c}\text { rRNA } \\
\text { target site } \\
\text { (E. coli } \\
\text { numbering) }\end{array}$ & Specificity & $\begin{array}{c}\text { Formamide } \\
\text { concentration } \\
\text { used in } \\
\text { hybridizations* }\end{array}$ \\
\hline EUB338 & GCTGCCTCCCGTAGGAGT & $16 \mathrm{~S}, 338$ & Most eubacteria & $0-50 \% * *$ \\
\hline $\begin{array}{l}\text { (Amann et } \\
\text { al. 1990) }\end{array}$ & Су3 & 355 & & \\
\hline ALF968 & GGTAAGGTTCTGCGCGTT & $16 S, 968-$ & $\alpha$-proteobacteria & $20 \%$ \\
\hline (Neef 1997) & Cy5 & 985 & & \\
\hline BET42a ${ }^{1}$ & GCCTTCCCACTTCGTTT & $23 \mathrm{~S}, 1027-$ & $\beta$-proteobacteria & $35 \%$ \\
\hline $\begin{array}{l}\text { (Manz et al. } \\
\text { 1992) }\end{array}$ & Сy5 & 1043 & & \\
\hline GAM42a ${ }^{1}$ & GCCTTCCCACATCGTTT & $23 \mathrm{~S}, 1027-$ & $\gamma$-proteobacteria & $35 \%$ \\
\hline $\begin{array}{l}\text { (Manz et al. } \\
\text { 1992) }\end{array}$ & Cy5 & 1043 & & \\
\hline SCB1 & CACTATCCGCGATTGGTA & $16 S, 997-$ & SCB Species 1 (See & $0-50 \% * * *$ \\
\hline (This study) & Cy5 & 1012 & Chapter 2) & \\
\hline SCB2 & CCGATGATGTCAAGGGTA & $16 \mathrm{~S}, 986-$ & SCB Species 2 (See & $0-50 \% * * *$ \\
\hline (This study) & Cy5 & 1003 & Chapter 2) & \\
\hline $\begin{array}{l}\text { NONEUB } \\
\text { (Wallner et } \\
\text { al. 1993) }\end{array}$ & $\begin{array}{c}\text { ACTCCTACGGGAGGCAGC } \\
\text { Су3 }\end{array}$ & $\begin{array}{c}\text { Does not } \\
\text { bind }\end{array}$ & $\begin{array}{l}\text { Nonsense probe } \\
\text { (Negative control for } \\
\text { non-specific binding) }\end{array}$ & $0-50 \% * * * *$ \\
\hline
\end{tabular}

See overleaf for table description. 
1 BET42a and GAM42a hybridizations were carried out with equal volumes of competitor probes (unlabeled GAM42a and BET42a, respectively). This was done to prevent non-specific binding, as both probes target the same site and differ by only one nucleotide (See Figure 5). * Formamide concentration in the hybridization buffer was the variable which was altered to adjust stringency (higher concentrations are more stringent). ** The formamide concentration used with the EUB338 probe varied depending on what probe it was used in conjunction with. When used alone, the formamide concentration was $35 \%$. $* * *$ Species-specific probes (SCB1 and SCB2) were hybridized using the following formamide concentrations: $0 \%, 20 \%, 35 \%$, and $50 \%$. As these hybridizations were unsuccessful, it is not possible to say what the optimal concentration is. **** As the NONEUB probe was used as a nonsense control to check for nonspecific binding, hybridizations were carried out at formamide concentrations matching each of those used with sense probes, i.e. $0 \%, 20 \%, 35 \%$, and $50 \%$.

Previously published probe sequences were obtained from probeBase (Loy et al. 2007), and further information can be obtained from the website: http://www.microbialecology.net/probebase. The three proteobacteria-specific probes were selected based on previous work which suggests that SCB belong to the phylum Proteobacteria (Lesser \& Blakemore 1990, Burnett \& McKenzie 1997), and the eubacterial probe EUB338 was selected to test more generally for the presence of SCB in the study species. Speciesspecific probes SCB1 and SCB2 were designed using the Probe Design tool in the phylogenetic package ARB (Ludwig et al. 2004). These probes were designed to target two of the bacteria identified in Chapter 2 as putative SCB of S. mollis (SCB species 1 and 2) and Patiriella sp. (SCB species 2). Both probes are specific to their target species when hybridized at the correct stringency, and are fairly robust to deviations from this optimum. A table showing their specificities at varying levels of hybridization stringency, expressed as deviations in hybridization temperature, can be found in the Appendix (Table A4). Figures $3.1-3.4$ illustrate the secondary structure of the $16 \mathrm{~S}$ rRNA of four bacteria and the areas targeted by four of the probes used in this study. Figure 3.5 shows the target region of the $\beta$ - and $\gamma$-proteobacteria-specific probes, BET42a and GAM42a, which target the 23S rRNA. 


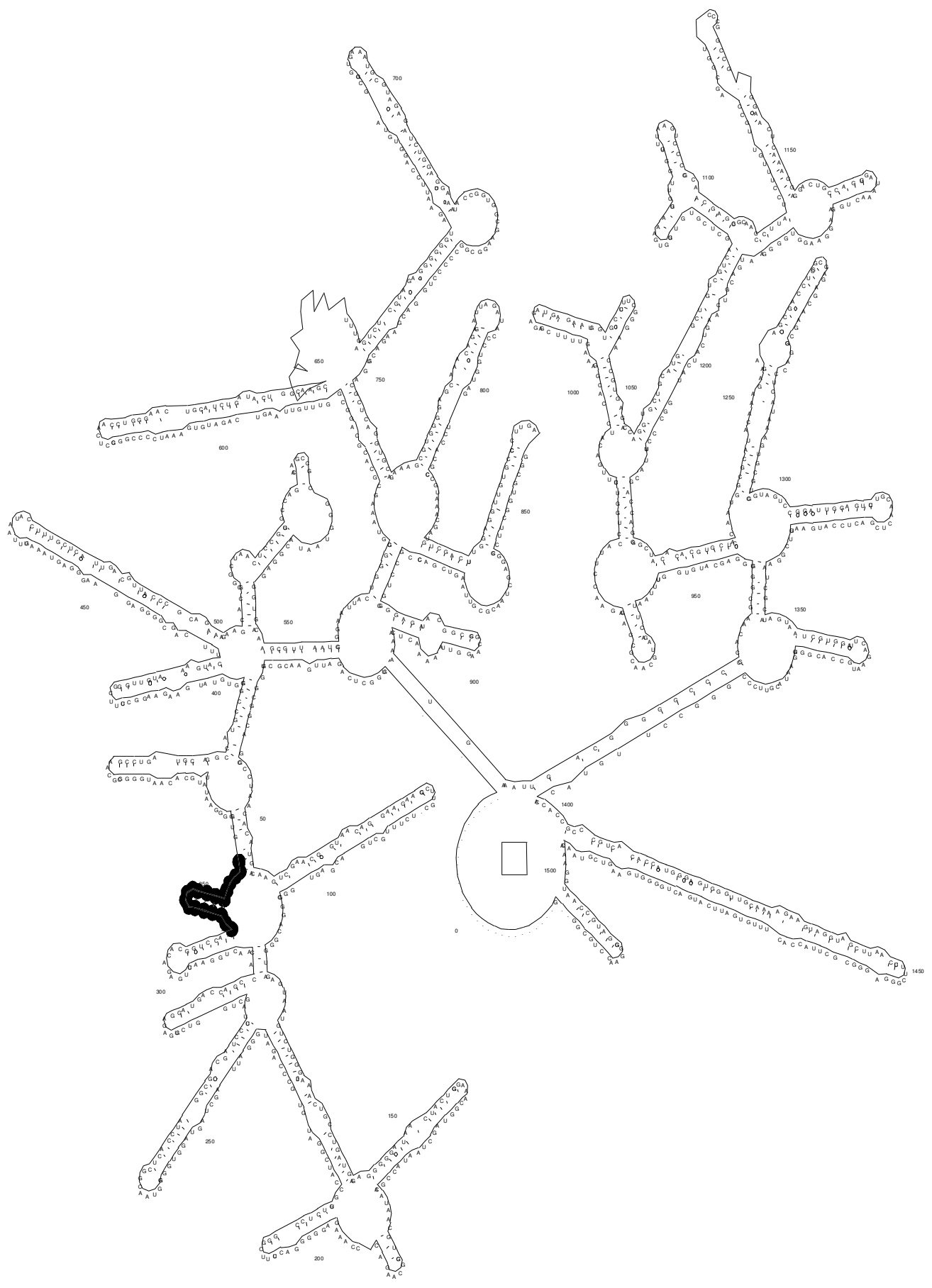

Figure 3.1. Secondary structure of the 16S rRNA of Escherichia coli, showing the binding site of the eubacterial FISH probe EUB338 (marked in black). The figure was produced using the secondary structure editor in the phylogenetic software package ARB. 


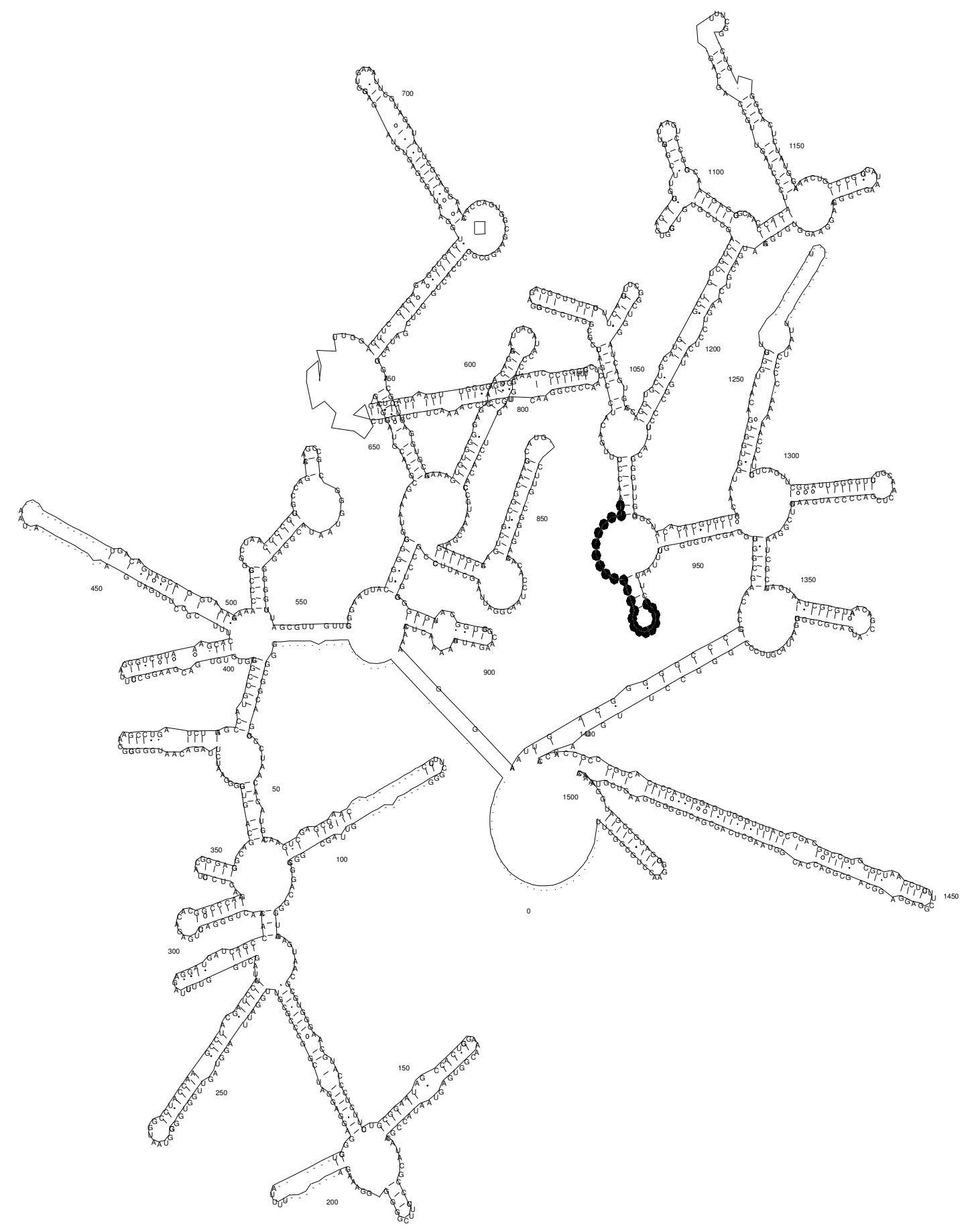

Figure 3.2. Secondary structure of the 16S rRNA of the $\alpha$-proteobacterium Roseovarius tolerans, showing the binding site of the alpha-proteobacteria specific FISH probe ALF968 (marked in black). The figure was produced using the secondary structure editor in the phylogenetic software package ARB. 


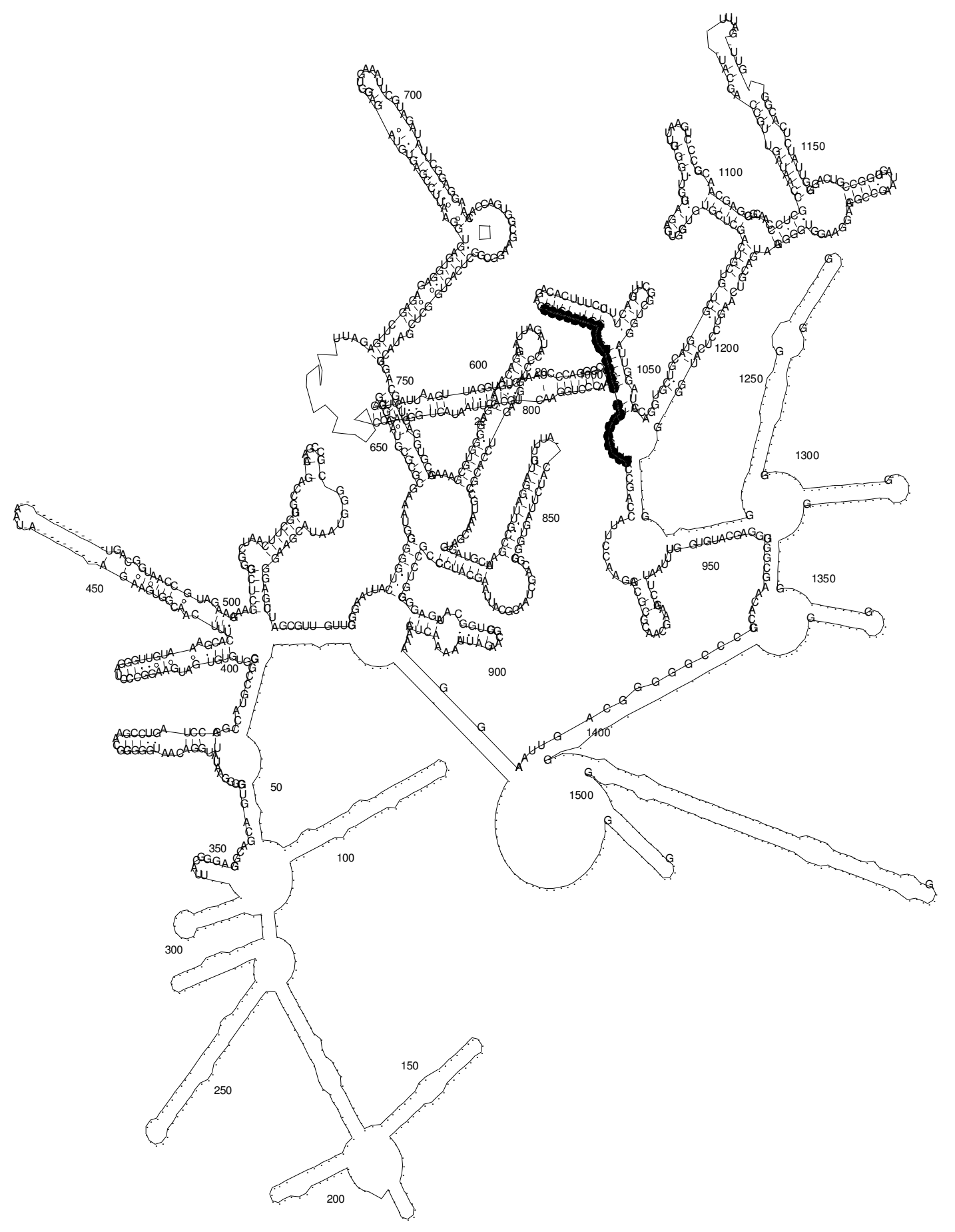

Figure 3.3. Secondary structure of part of the $16 \mathrm{~S}$ rRNA of SCB species 1, showing the binding site of the species-specific FISH probe SCB1 (shown in black). The figure was produced using the secondary structure editor in the phylogenetic software package ARB. 


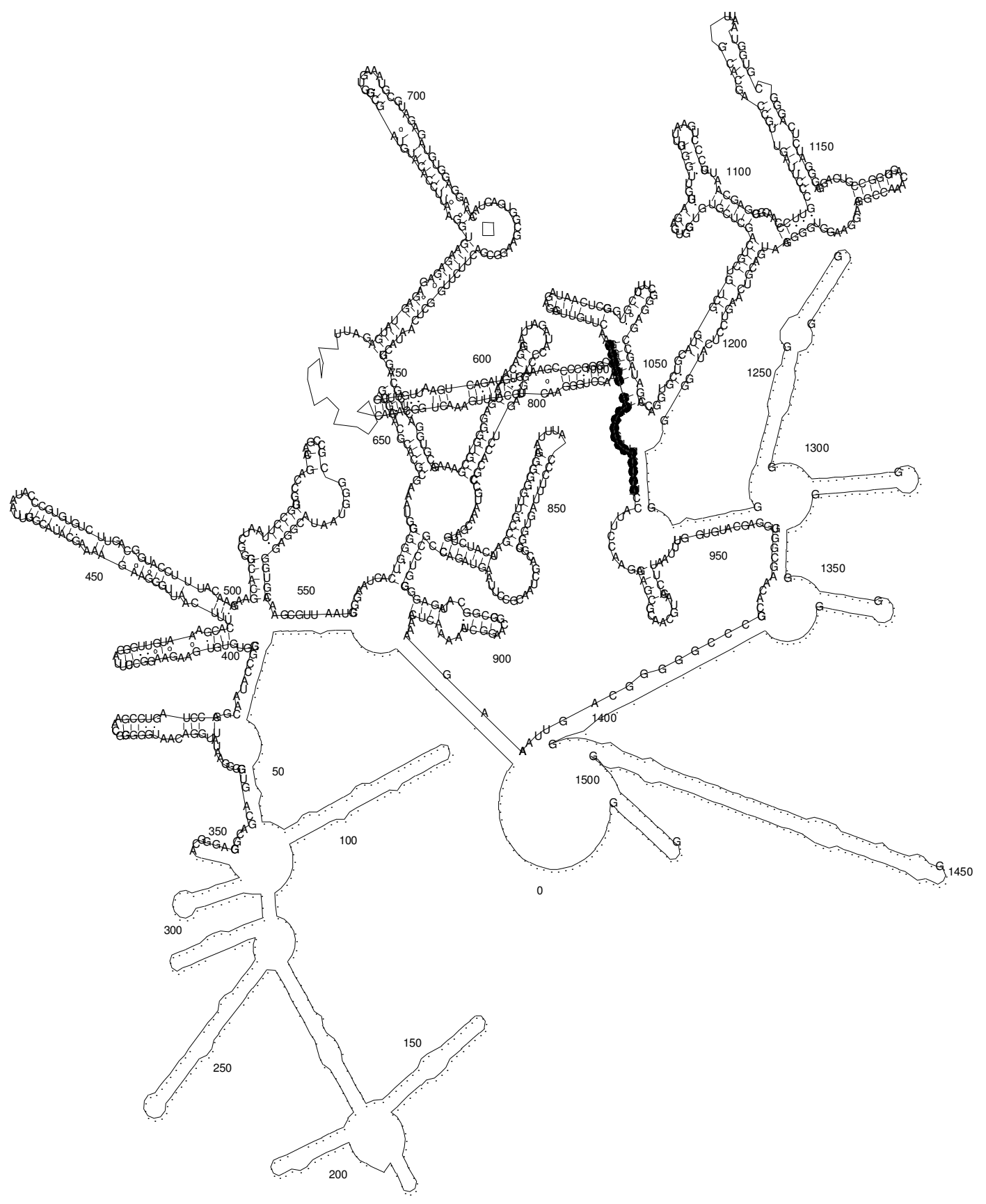

Figure 3.4. Secondary structure of part of the 16S rRNA of SCB species 2, showing the binding site of the species-specific FISH probe SCB2 (shown in black). The figure was produced using the secondary structure editor in the phylogenetic software package ARB. 


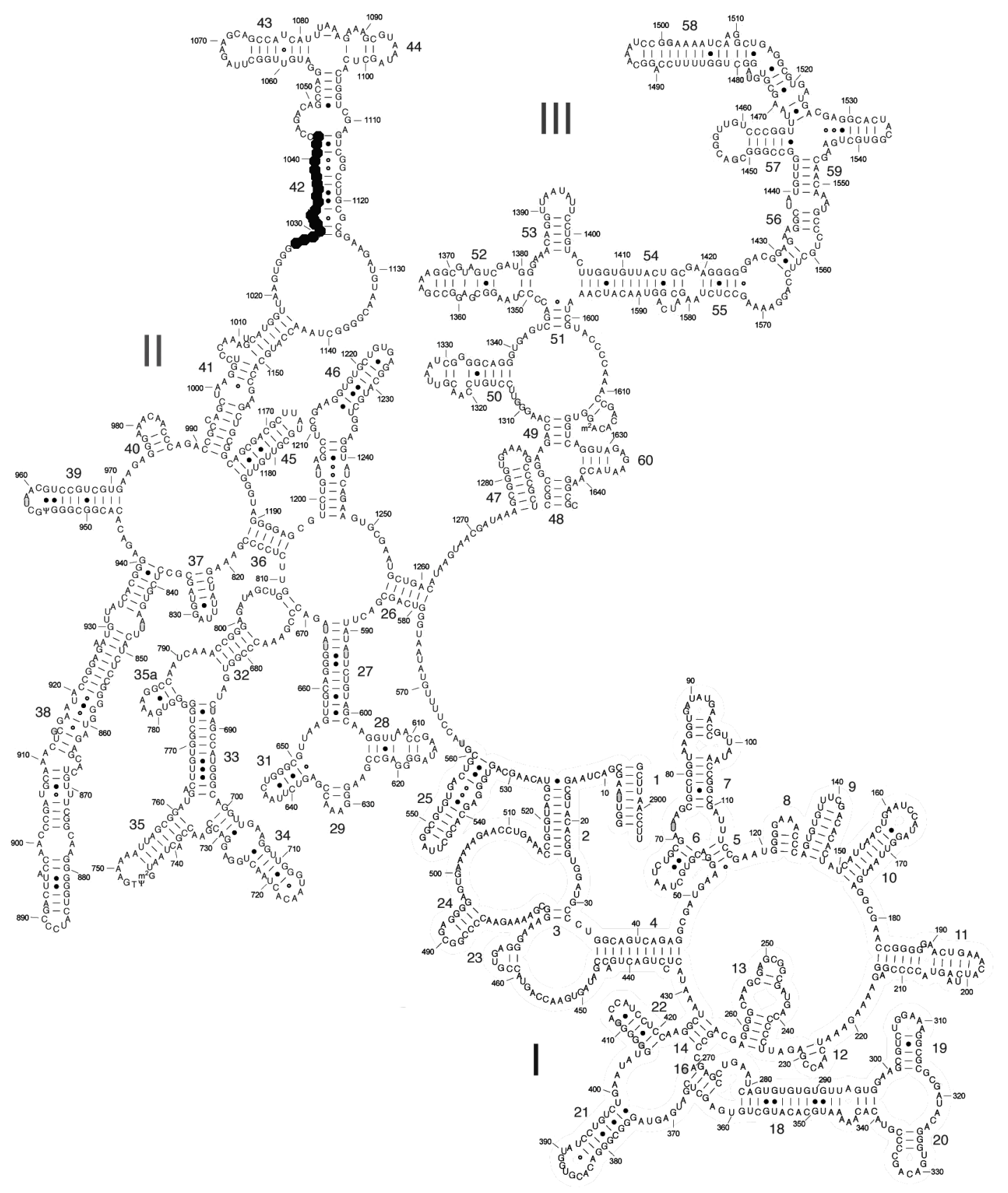

Figure 3.5. Secondary structure of the $23 \mathrm{~S}$ rRNA of Escherichia coli showing the binding site of the $\gamma$ proteobacteria-specific FISH probe GAM42a (shown in black). This is also the binding site of the probe BET42a in $\beta$-proteobacteria. Figure modified from that on the website of the Center of Molecular Biology of RNA, University of California, Santa Cruz (http://rna.ucsc.edu). 


\section{Experimental design}

The three objectives of the FISH assays were 1) To determine the identity of SCB to as high a resolution as possible (i.e. preferably to species level), 2) To determine what proportion of the SCB community is made up of the particular bacteria identified, and 3) To make observations on the morphology of the SCB.

To meet the first objective, hybridizations were carried out on all three echinoderm species, using the full range of probes one at a time. The eubacterial probe (EUB338) was the first to be used, in order to test for the presence of SCB in each species. Those species which tested positive for the presence of SCB were then hybridized with the three proteobacterial probes (ALF968a, BET42a, GAM42a), one probe at a time, to test the hypothesis that SCB belong to the phylum Proteobacteria, and more specifically, to determine which subdivision(s) they fall into. Finally, the more specific probes SCB1 and SCB2 were used in an attempt to verify results of earlier phylogenetic work (See Chapter 2) and identify the SCB of these species at the highest resolution possible. For each hybridization, at least 10 randomly-selected areas of the cuticle from two individuals of each echinoderm species were viewed in order to reduce the chances of particular SCB being overlooked. Negative controls, consisting of unlabeled (i.e. non-hybridized) tissue sections, and tissue sections hybridized with the NONEUB probe (to test for non-specific binding), were also included with each hybridization.

In order to meet the second objective, hybridizations were carried out using the eubacterial probe EUB338 in conjunction with one or more other probes. As the 
eubacterial probe should bind to all SCB present in the sample, its use with other, more specific probes can provide an indication of what proportion of the SCB community is made up of those bacteria targeted by the more specific probe. For example, if EUB338 (Cy3-labeled) is used with ALF968 (Cy5-labeled), all $\alpha$-proteobacteria will fluoresce at both the $\mathrm{Cy} 3$ and $\mathrm{Cy} 5$ wavelengths, while those bacteria which are not $\alpha$-proteobacteria will only fluoresce at the $\mathrm{Cy} 3$ wavelength. Table 3.2 provides details of the hybridizations which were performed. For each hybridization, at least 10 randomlyselected areas of the cuticle from two individuals of each echinoderm species were viewed in order to estimate the abundance of the bacteria bound by the different probes. Negative controls were included, and consisted of unlabelled and NONEUB-hybridized tissue sections as described earlier.

Table 3.2. FISH probe combinations used to determine SCB community composition

\begin{tabular}{cc}
\hline Probe combination* & To determine proportion of SCB made up by... \\
\hline EUB338 + ALF968 + GAM42a & $\alpha$ - and $\gamma$-proteobacteria combined \\
EUB338 + ALF968 & $\alpha$-proteobacteria \\
EUB338 + SCB1 & $\gamma$-proteobacteria \\
EUB338 + SCB2 & SCB species 1 \\
\end{tabular}

* As BET42a was found in earlier assays not to bind in any samples, it was not included in this group of hybridizations.

To facilitate observations of SCB morphology, both two-dimensional and threedimensional images were captured using a confocal laser scanning microscope (CLSM) (see next section). The three-dimensional images, created by serial optical sectioning of 
tissue sections by the CLSM, were particularly useful for determining SCB morphology, as the SCB within an individual echinoderm often appeared to all be orientated in a similar direction, making it difficult to determine their shape and size from twodimensional images alone.

\section{Confocal laser scanning microscopy}

In order to visualize the results of hybridizations, an Olympus FluoView FV1000 confocal laser scanning microscope (CLSM) was used. The different dyes and stains used (Cy3, Cy5 and DAPI) are stimulated by different wavelength lasers on the CLSM. In addition to these dyes and stains, the echinoderm tissues themselves autofluoresced at certain wavelengths. Autofluorescence was particularly strong at the wavelength corresponding to the dye fluorescein isothiocyanate (FITC) (assessed by conventional epifluorescence microscopy). This autofluorescence was utilized as a sort of 'stain' for the echinoderm tissue sections which were not stained with DAPI. Figures 3.6 - 3.9 show the excitation and emission spectra for Cy3, Cy5, DAPI and FITC, respectively, and the wavelengths of the lasers used to excite each. 


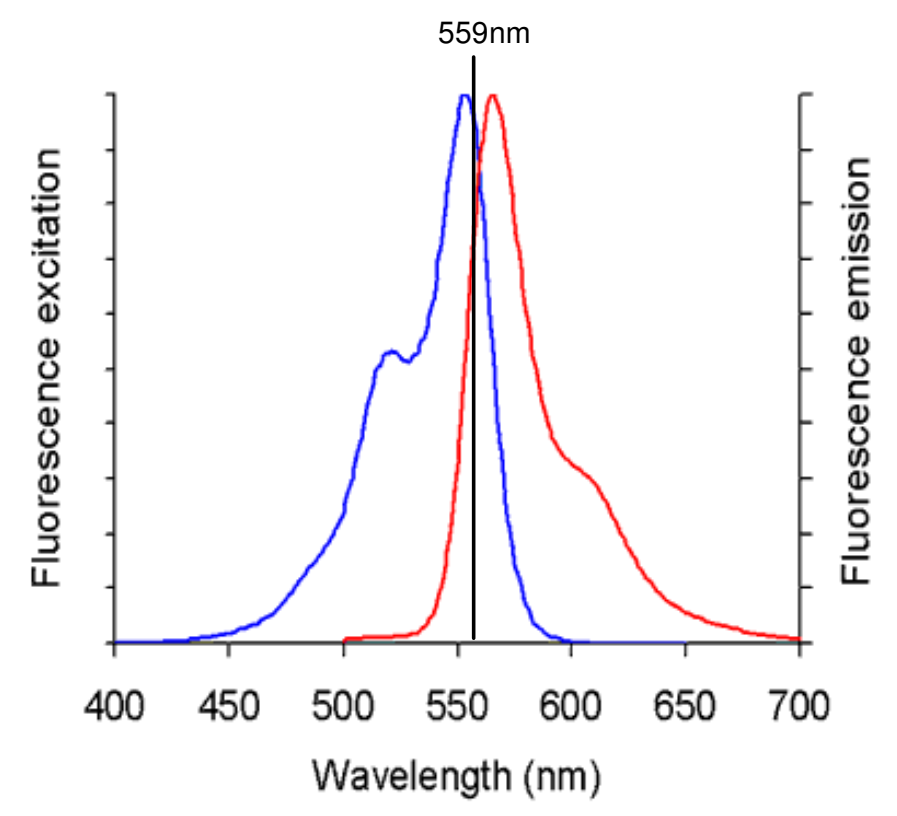

Figure 3.6. Excitation and emission spectra for the dye Cy3. Blue: excitation spectrum, Red: emission spectrum. $559 \mathrm{~nm}$ line: wavelength of laser used in CLSM to excite dye. Image reproduced from Invitrogen (www.invitrogen.com).

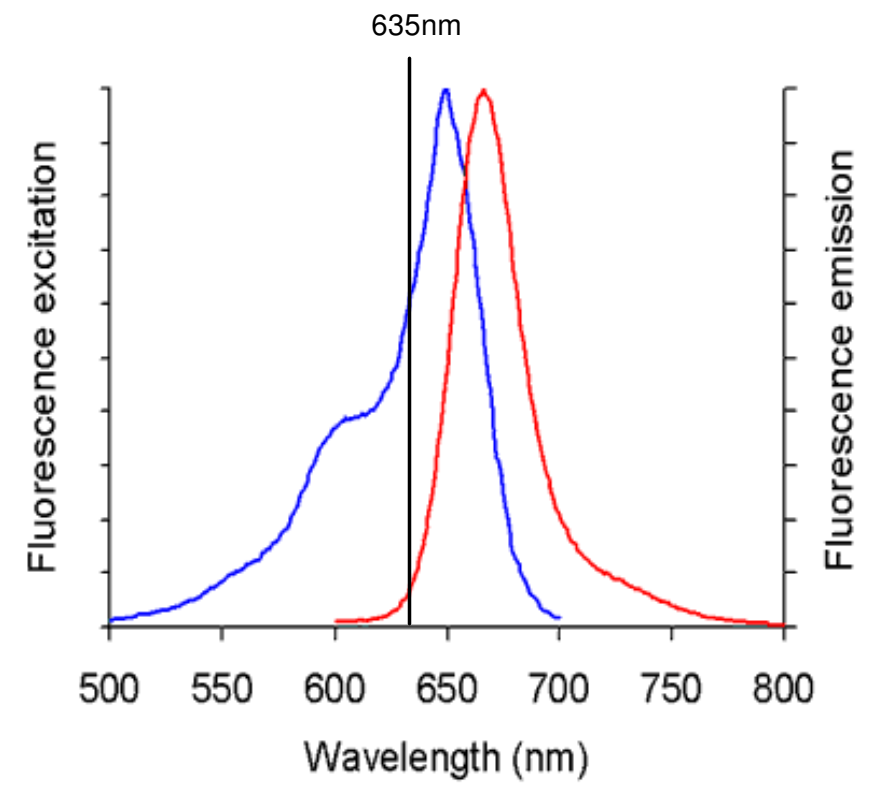

Figure 3.7. Excitation and emission spectra for the dye Cy5. Blue: excitation spectrum, Red: emission spectrum. $635 \mathrm{~nm}$ line: wavelength of laser used in CLSM to excite dye. Image reproduced from Invitrogen (www.invitrogen.com). 


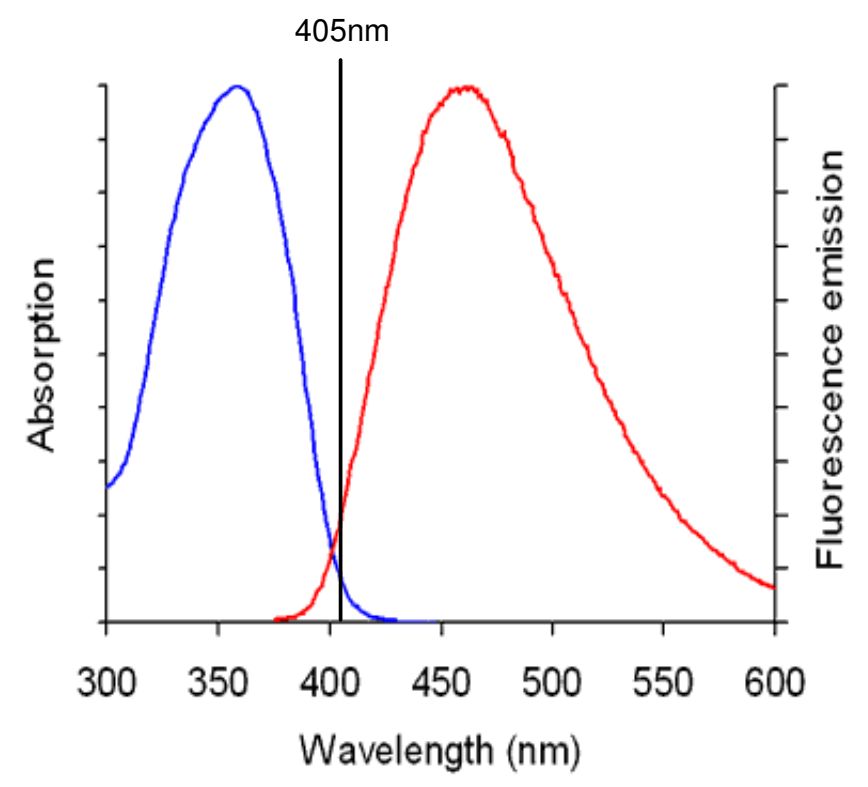

Figure 3.8. Excitation and emission spectra for the DNA stain DAPI. Blue: excitation spectrum, Red: emission spectrum. $405 \mathrm{~nm}$ line: wavelength of laser used in CLSM to excite dye. Image reproduced from Invitrogen (www.invitrogen.com).

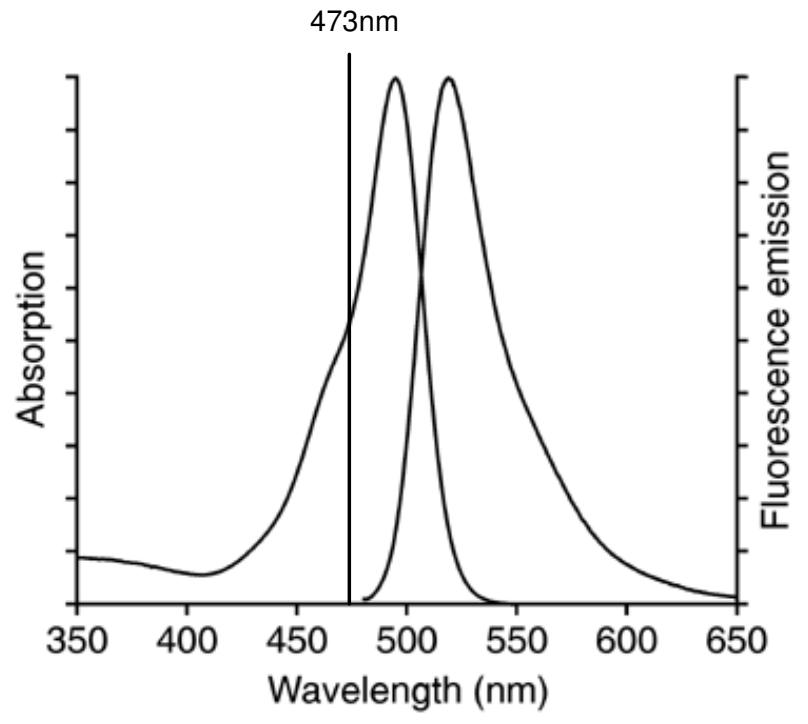

Figure 3.9. Excitation and emission spectra for the dye FITC. Left curve: excitation spectrum, Right curve: emission spectrum. $473 \mathrm{~nm}$ line: wavelength of laser used in CLSM to induce autofluorescence of echinoderm tissues. Image reproduced from Invitrogen (www.invitrogen.com). 
As well as autofluorescing strongly under the $473 \mathrm{~nm}$ laser, the tissue sections all autofluoresced to a lesser extent under each of the other three lasers used. To counteract this autofluorescence, unlabelled tissue sections (which had been through all processing and hybridization stages, but without the addition of probes), were used to calibrate the settings of the CLSM. This involved viewing the unlabeled slides at $1,000 \mathrm{x}$ magnification, and adjusting CLSM settings such as laser intensity, detector high voltage (HV) and gain, until the desired levels of fluorescence were attained. The levels of fluorescence were deemed appropriate when the fluorescence from the $405 \mathrm{~nm}$ or $473 \mathrm{~nm}$ laser (corresponding to DAPI and FITC) was bright enough to be seen clearly, and the fluorescence from the $559 \mathrm{~nm}$ or $635 \mathrm{~nm}$ laser (corresponding to Cy3 and Cy5 - FISH probe dyes) was just below the level of detection. Several unlabelled sections from each echinoderm species were tested this way to ensure that the results were consistent across samples. The settings which were established during this calibration were kept constant when viewing all labeled (i.e. hybridized with FISH probes) tissue sections. By calibrating the CLSM in this way, it was possible to say with some certainty that strong fluorescence produced by the $559 \mathrm{~nm}$ and $635 \mathrm{~nm}$ lasers in labeled tissue sections did actually indicate the presence of bacteria, rather than autofluorescence of the echinoderm tissue. Figure 3.10 provides examples of unlabelled tissue sections that were calibrated in this manner. 

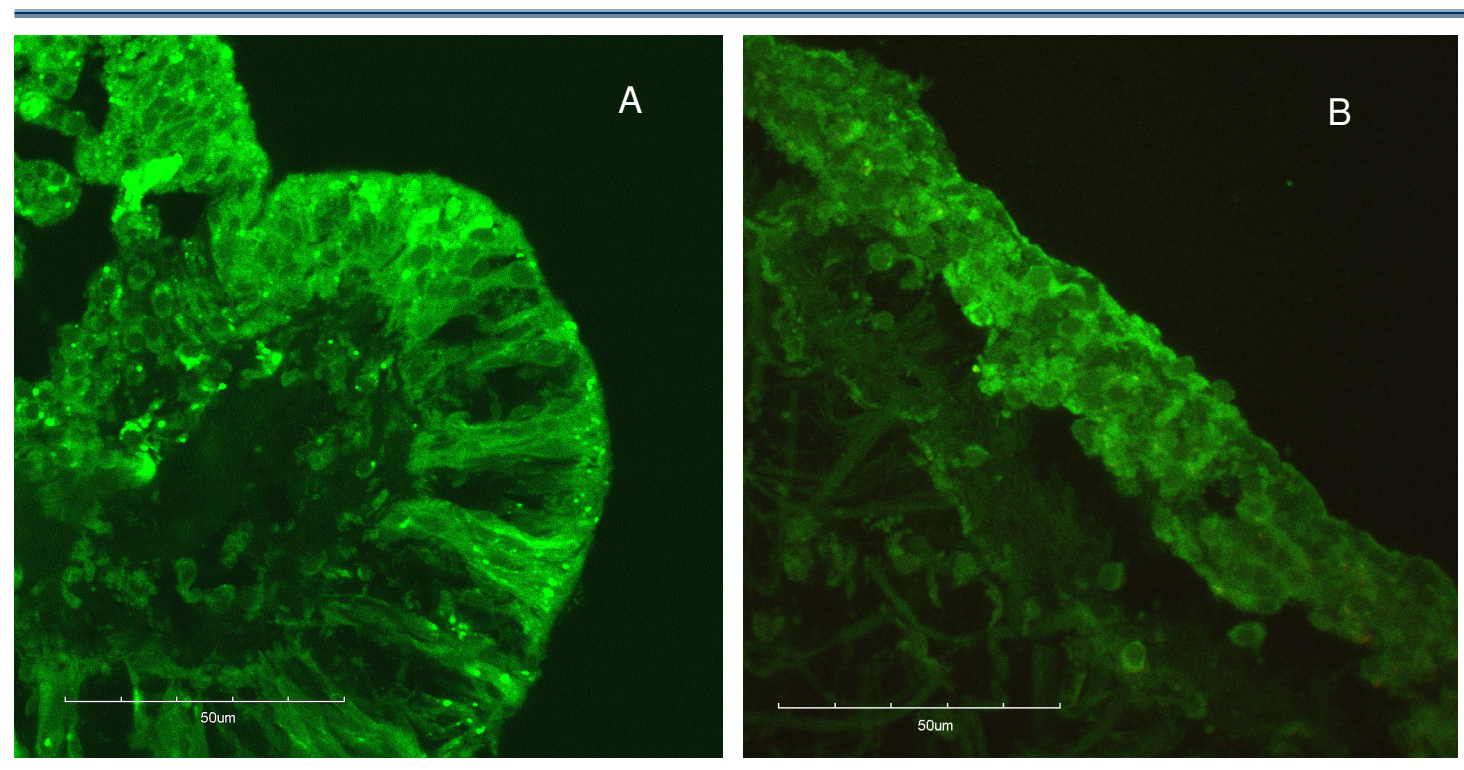

Figure 3.10. Unlabeled echinoderm sections, showing the autofluorescence of the tissue. A: Patiriella sp. Laser wavelengths used were $473 \mathrm{~nm}$ and $559 \mathrm{~nm}$. B: Stichopus mollis. Laser wavelengths used were 473 $\mathrm{nm}$ and $635 \mathrm{~nm}$.

\section{CARD-FISH}

In addition to the standard FISH assays mentioned above, Catalyzed Reporter Deposition (CARD) FISH assays were carried out by Dr. Michael Taylor at the School of Biological Sciences, University of Auckland. CARD-FISH utilizes tyramide signal amplification to provide increased signal intensities from hybridized cells, especially in cases where the target bacteria are small or slow-growing (See Pernthaler et al. (2002) for a description of the method). CARD-FISH was performed using the eubacterial probe EUB338, labeled with fluorescein isothiocyanate (FITC). Hybridizations were carried out as follows. Tissue sections were prepared and placed on to slides as described earlier for standard FISH assays. Slides were then dipped in $0.2 \%$ agarose and air dried at $35{ }^{\circ} \mathrm{C}$ for 10 minutes. $29 \mu \mathrm{l}$ proteinase $\mathrm{K}\left(15 \mu \mathrm{g} \mathrm{ml}^{-1}\right)$ was then added to wells drawn on each slide, and incubated at room temperature for 10 minutes. Slides were washed in $50 \mathrm{ml}$ water, then incubated in $0.01 \mathrm{M} \mathrm{HCl}$ at room temperature for 10 minutes, to bleach endogenous 
peroxidase. After incubation, slides were washed in $50 \mathrm{ml}$ water and air dried. Hybridization buffer (see Appendix Table A5 for formula) and probe (50 ng $\mu^{-1}$ ) were mixed in a 300:1 ratio, $10 \mu \mathrm{l}$ of the mixture was added to slides, and slides were incubated in a hybridization chamber containing $2 \mathrm{ml}$ hybridization buffer at $46{ }^{\circ} \mathrm{C}$ for 2 hours. Following hybridization, slides were transferred to tubes containing pre-warmed wash buffer (see Appendix Table A6 for formula) and incubated at $48{ }^{\circ} \mathrm{C}$ for 15 minutes. Tyramide signal amplification was achieved using the following protocol. Hybridized slides were briefly dipped in $50 \mathrm{ml}$ water to remove salts, then incubated in $1 \mathrm{x}$ PBS at room temperature for 15 minutes. Slides were dried slightly by dabbing on blotting paper, then incubated in substrate mix (1 part FITC-labeled tyramide, 500-2000 parts amplification buffer (see Appendix Table A7 for formula) and $0.0015 \%$ hydrogen peroxide) at $46{ }^{\circ} \mathrm{C}$ for 1 hour in the dark. Slides were subsequently removed and dabbed dry on blotting paper. Slides were then washed as follows: 10 minutes in $1 \mathrm{x}$ PBS, followed by $50 \mathrm{ml}$ water then $50 \mathrm{ml} 96 \%$ ethanol. All washes were carried out at room temperature in the dark. After washing, slides were air dried, and counter-stained with DAPI. DAPI staining consisted of pipetting drops of DAPI (1-5 $\left.\mu \mathrm{g} \mathrm{ml}^{-1}\right)$ into wells, incubating for 3 minutes in the dark, then washing in water for 15 seconds and $80 \%$ ethanol for 1 minute. Once dry, coverslips were mounted using Citifluor pH 9 mounting medium. Results were visualized using an Olympus BH2 BHS epifluorescence microscope at the University of Auckland and an Olympus Fluoview FV-1000 CLSM at Victoria University of Wellington. 


\section{Image manipulation}

Image manipulation was kept to a minimum in order to preserve the appearance of the original image produced by the CLSM as much as possible. The only adjustments made to images were increases in brightness and contrast in order to make viewing easier in the printed form. Brightness and contrast adjustments were kept constant across all images in order to eliminate any bias in the results. When making counts of SCB to determine the proportion of the community made up by a particular group, an edge-detect filter was applied to images, to make detection of individual bacteria easier. Image adjustments were performed using the Gnu Image Manipulation Program (GIMP).

\subsection{Results}

\section{Identification of SCB}

SCB were found in the cushion star Patiriella sp. and the sea cucumber S. mollis, but were never found in the sea star A. scabra (bacteria were observed within the tissue just below the epidermal layer in A. scabra however). Through the use of $\alpha-\beta$-, and $\gamma$ proteobacteria specific FISH probes, it was found that SCB belonging to the $\alpha$ - and $\gamma$ proteobacteria were present in Patiriella sp. and S. mollis. Stichopus mollis appears to contain SCB belonging to both the $\alpha$ - and $\gamma$-proteobacteria, while only $\alpha$-proteobacteria were found in Patiriella sp. In S. mollis, $\alpha$-proteobacteria were found much more often than $\gamma$-proteobacteria, and in much higher abundance (usually $\alpha$-proteobacteria were three or four times as abundant as $\gamma$-proteobacteria). In all sections examined, the distribution of SCB within an individual was found to be very patchy, with certain areas rich in SCB, 
and others completely devoid of SCB. Areas where the epidermis pinches inwards were found to often have high numbers of SCB. The species-specific probes SCB1 and SCB2 both failed to produce positive results. Whether this was because they failed to hybridize or because the target bacteria were not present is not known. The results of these hybridizations are summarized in Table 3.3, and representative images of all successful hybridizations are presented in Figures $3.11-3.13$

Table 3.3. Results of FISH assays, carried out using each probe individually

\begin{tabular}{cccc}
\hline Probe & \multicolumn{3}{c}{ Echinoderm species } \\
& Patiriella sp. & S. mollis & A. scabra \\
\hline EUB338 (Eubacteria) & + & + & - \\
ALF968 ( $\alpha$-proteobacteria) & + & + & - \\
BET42a ( $\beta$-proteobacteria) & - & - & - \\
GAM42a ( $\gamma$-proteobacteria) & - & + & - \\
SCB1 (SCB species 1) & - & - & - \\
SCB2 (SCB species 2) & - & - & \\
\hline “" PrObe succesfully hybidize & & & - \\
\hline
\end{tabular}

"+"= Probe successfully hybridized. -= Probe did not successfully hybridize. 

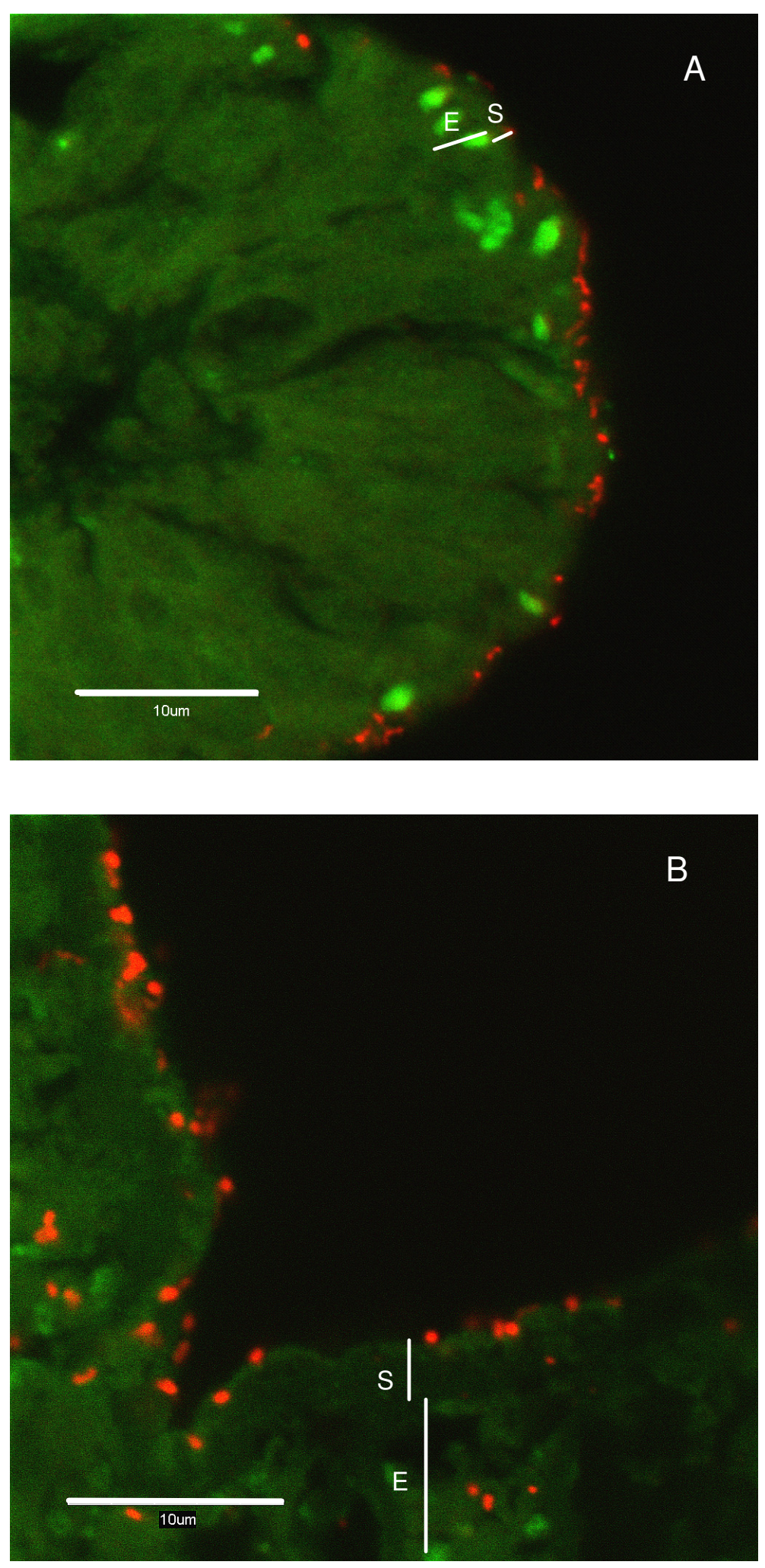

Figure 3.11. CLSM images of EUB338 hybridizations. Full description of figure overleaf. 


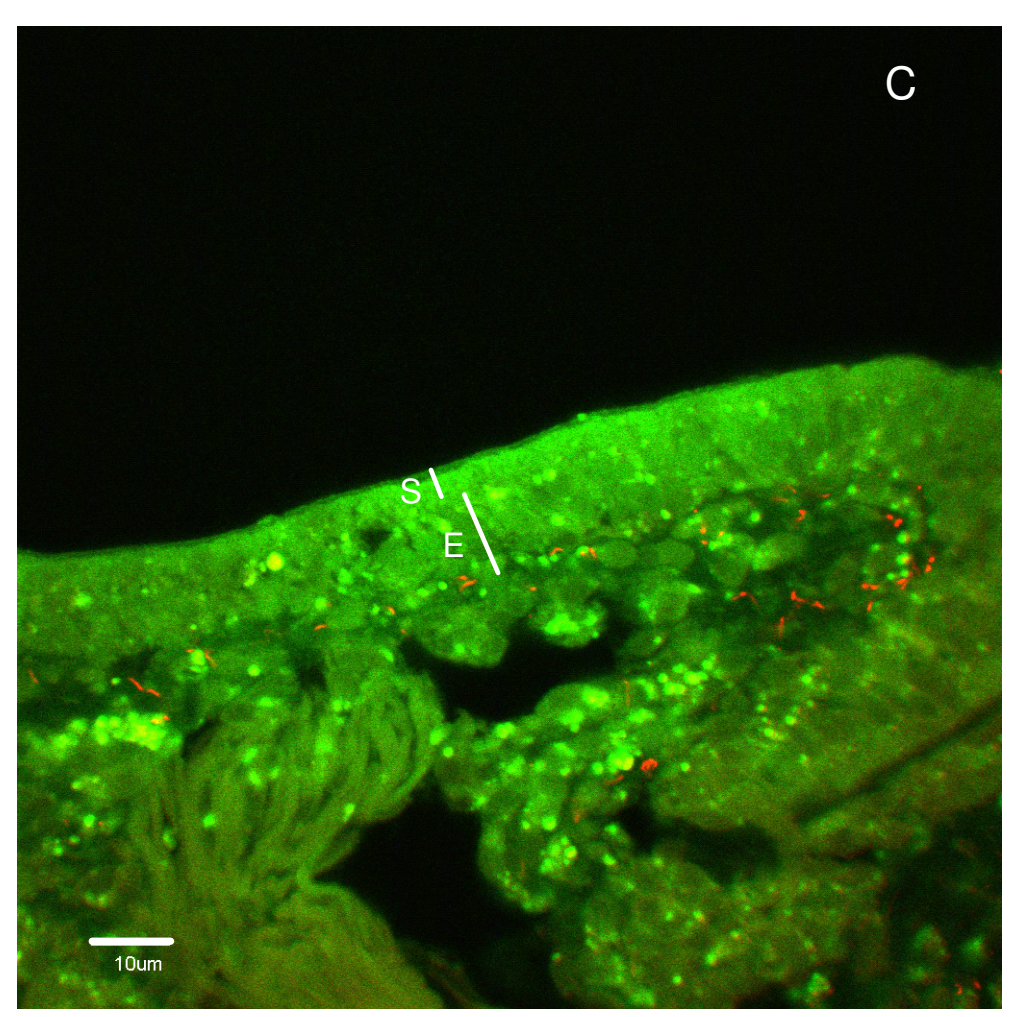

Figure 3.11. CLSM images of EUB338 hybridizations. Fluorescently-labeled bacteria are shown in red. A: Patiriella sp., B: Stichopus mollis, C: Astrostole scabra. Note the absence of bacteria in the epidermal and cuticular areas in $\mathrm{C}$, and the presence of larger rod-shaped bacteria deeper within the tissue. In all images, $\mathrm{S}$ $=$ approximate size of sub-cuticular space, $\mathrm{E}=$ Approximate size of epidermal cell layer. All scales bars represent $10 \mu \mathrm{m}$. 

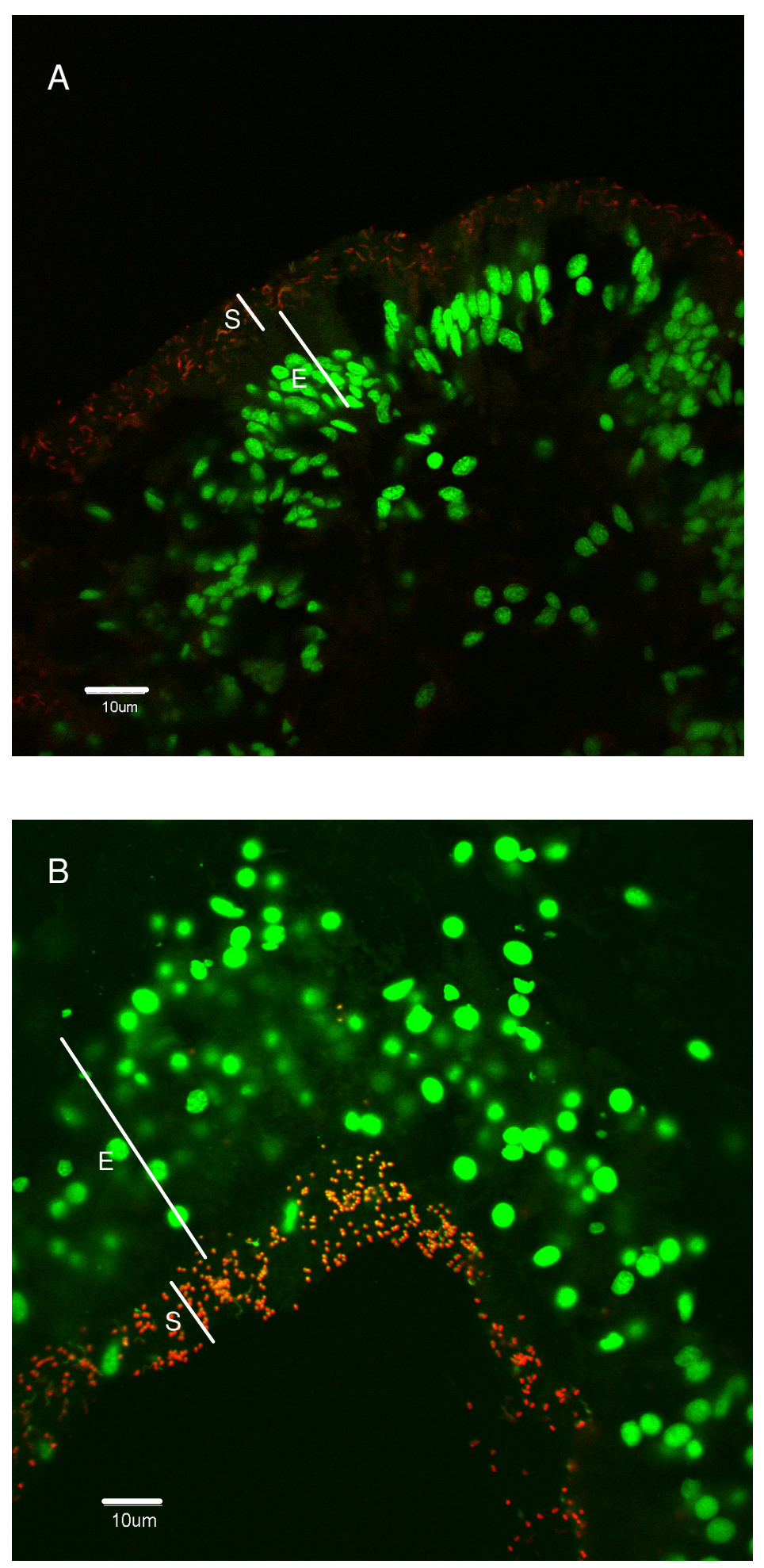

Figure 3.12. CLSM images of ALF968 hybridizations. Fluorescently-labeled bacteria are shown in red. A: Patiriella sp., B: S. mollis. Note the brightly-fluorescing green DAPI-stained nuclei belonging to the echinoderm epidermal cells, beneath the sub-cuticular space where the SCB can be seen. In both images, S $=$ approximate size of sub-cuticular space, $\mathrm{E}=$ approximate size of epidermal cell layer. Scale bars represent $1 \mu \mathrm{m}$. 


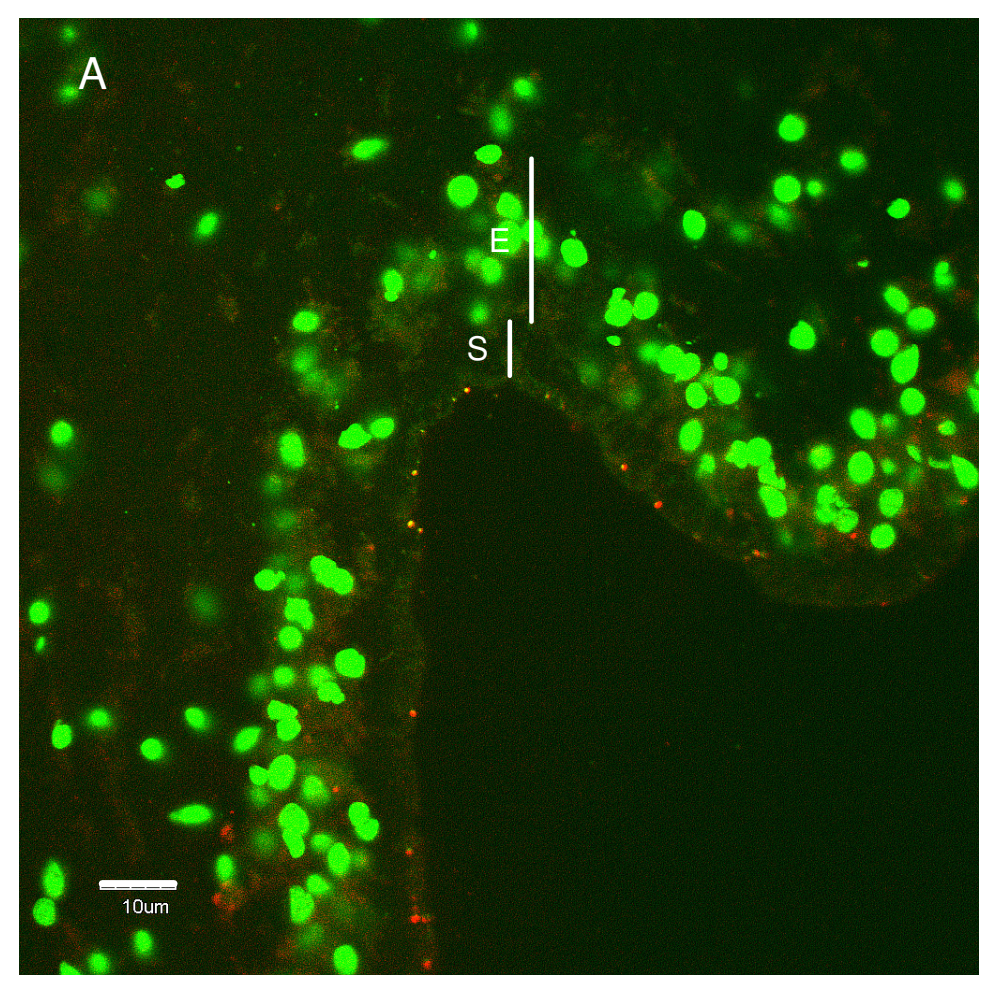

Figure 3.13. CLSM image of GAM42a hybridization in Stichopus mollis. Fluorescently-labeled bacteria are shown in red. Sample has also been DAPI-stained (note the brightly-fluorescing green epidermal cell nuclei below the sub-cuticular space containing the SCB). S = approximate size of sub-cuticular space, $\mathrm{E}=$ approximate size of epidermal cell layer. Scale bar represents $10 \mu \mathrm{m}$. Note the low number of SCB present in comparison to the ALF968-labelled bacteria shown in Figure 3.12B.

\section{SCB community composition}

As explained in the Materials and Methods section, an edge-detect filter was applied to CLSM images in GIMP, in order to facilitate easier counting. Bacteria hybridized with the eubacterial probe were labeled red, and those hybridized with more specific probes were labeled in blue. Where an overlap in colour occurred, producing a pink-purple colour, hybridization of both probes was assumed to have occurred. By calculating how many pink-purple bacteria and how many red bacteria were present, an approximate 
figure could be attained for the proportion of the SCB community made up by the bacterial group targeted by the more specific probe. An example of an image used for assessment of SCB community makeup is presented in Figure 3.14. In some cases, especially in tissue sections from Patiriella sp., the individual bacteria were too indistinct to tell apart, even after applying this filter. Such images were excluded from the analysis of SCB community composition. 

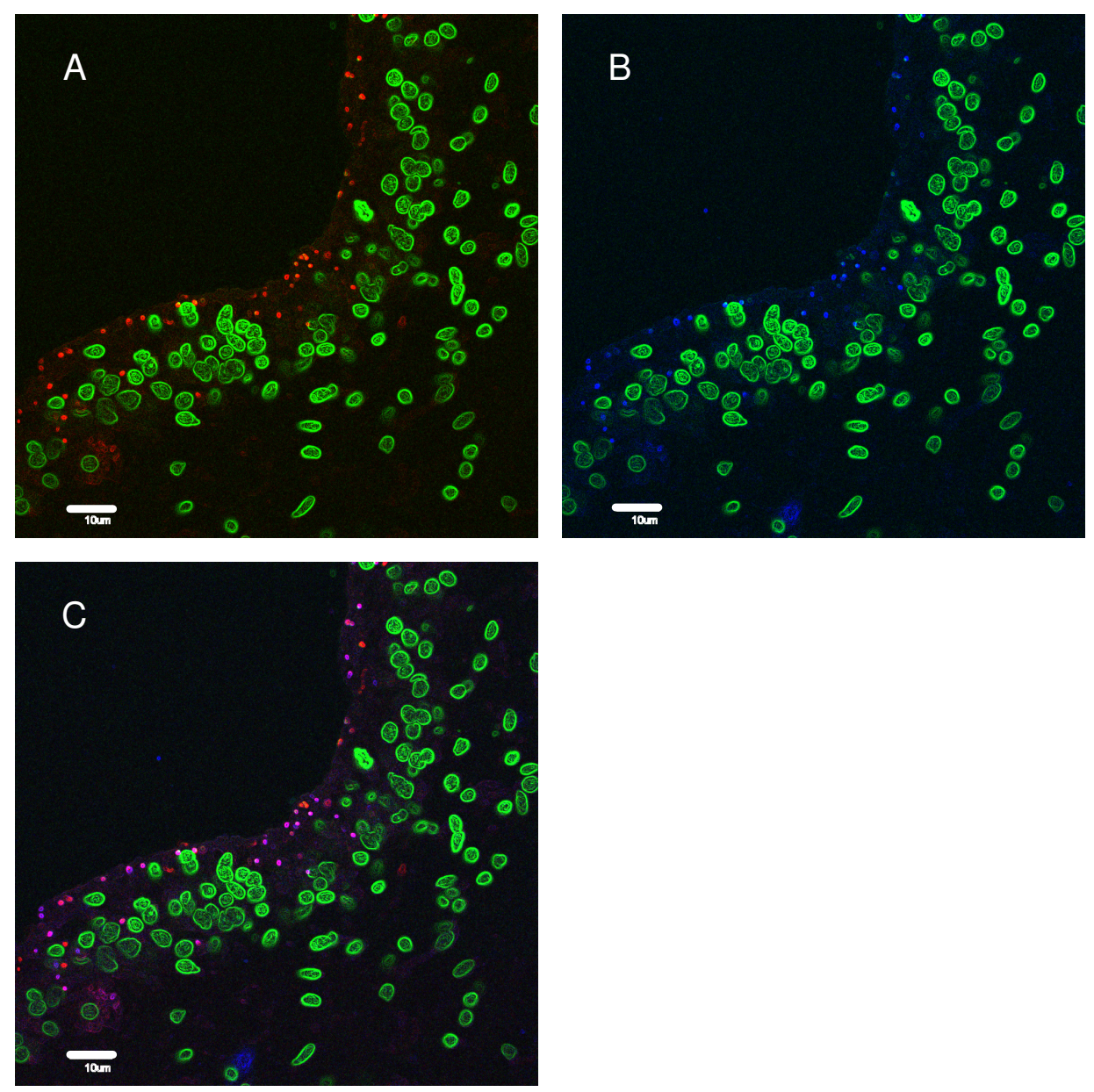

Figure 3.14. CLSM images of bacteria hybridized with EUB338, ALF968 and GAM42a probes. The tissue section is from Stichopus mollis. Images had an edge-detect filter applied in order to facilitate easier counting of bacteria. A: EUB338-hybridized bacteria (shown in red). B: ALF968+GAM42a-hybridized bacteria (shown in blue). C: Images A and B overlaid. Note the predominance of pink and purple coloured bacteria, indicating successful hybridization of the eubacterial and proteobacterial probes. Red-coloured bacteria in image $C$ represent those which only hybridized with the EUB338 probe, and therefore may be bacteria which do not belong to the proteobacteria. Scale bars represent $10 \mu \mathrm{m}$. 
A range of hybridizations were carried out, as described in Table 3.2. From the results of these hybridizations, it appears that members of the phylum Proteobacteria do in fact make up the vast majority of the SCB community in both Patiriella sp. and S. mollis. More specifically, the $\alpha$-proteobacteria seems to be the dominant group, with the $\gamma$ proteobacteria making up a small proportion of the community in S. mollis only. As was the case in the earlier FISH assays, both species-specific probes (SCB1 and SCB2) failed to hybridize. The results of these assays are presented in Tables 3.4 and 3.5.

Table 3.4. Proportion of SCB communities of Patiriella sp. and Stichopus mollis made up of $\alpha$ - and $\gamma$ proteobacteria combined.

\begin{tabular}{ccccc}
\hline $\begin{array}{c}\text { Echinoderm } \\
\text { species }\end{array}$ & $\begin{array}{c}\text { EUB338 } \\
\text { positive* }\end{array}$ & $\begin{array}{c}\text { ALF968+GAM42a } \\
\text { positive* }\end{array}$ & $\begin{array}{c}\text { \% of SCB made } \\
\text { up by } \boldsymbol{\alpha} \text { - and } \boldsymbol{\gamma} \text { - } \\
\text { proteobacteria }\end{array}$ & $\begin{array}{c}\text { \% of SCB made } \\
\text { up by other } \\
\text { bacteria }\end{array}$ \\
\hline $\begin{array}{c}\text { Patiriella } \text { sp. } \\
\text { S. mollis }\end{array}$ & 488 & 466 & $95.5 \%$ & $4.5 \%$ \\
& 538 & 458 & $85.1 \%$ & $14.9 \%$ \\
\hline
\end{tabular}

\footnotetext{
*Total number of bacteria considered to have successfully hybridized with listed probe(s), based on colour of the bacteria in CLSM images.
} 
Table 3.5. Proportion of SCB communities of Patiriella sp. and Stichopus mollis made up of $\alpha$ - and $\gamma$ proteobacteria.

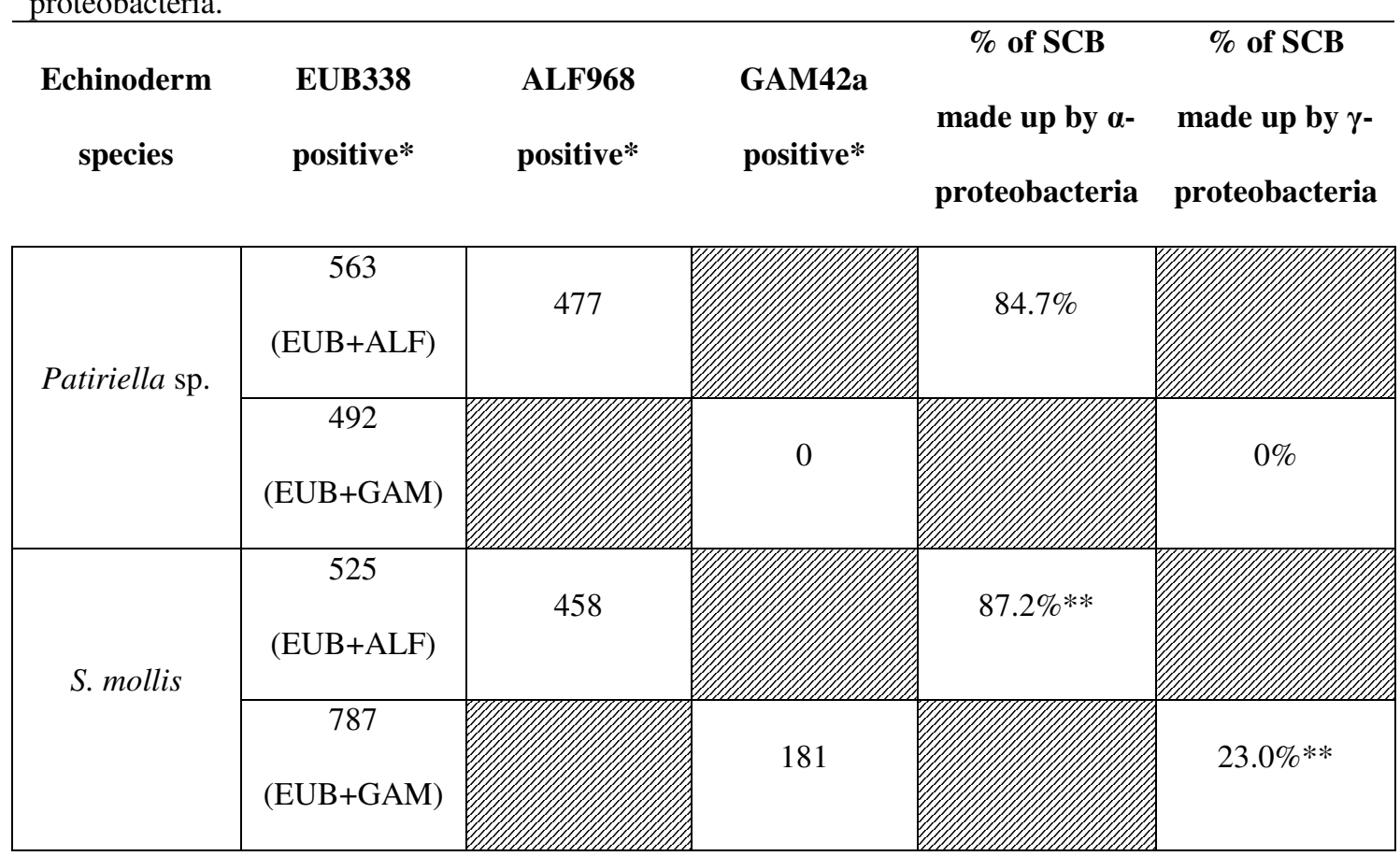

*Total number of bacteria considered to have successfully hybridized with listed probe(s), based on colour of the bacteria in CLSM images. **Percentages of $\alpha$ - and $\gamma$-proteobacteria in S. mollis do not equal 100, presumably because community composition varied between the different samples used for the EUB338 + ALF968 and EUB338 + GAM42a hybridizations.

\section{SCB morphology}

SCB were often found clumped together in large numbers, making observation of their morphology somewhat difficult. In many cases, SCB were also found to align themselves in such a way that in two-dimensional images they appeared as small cocci (see Figures 12-14 for example). By serially sectioning tissue sections with the CLSM to create threedimensional images, it was determined that the majority of SCB in Patiriella sp. and $S$. mollis are in fact bacilli rather than cocci. Figures 3.15 and 3.16 are images taken from three-dimensional animations, showing SCB in longitudinal section. 

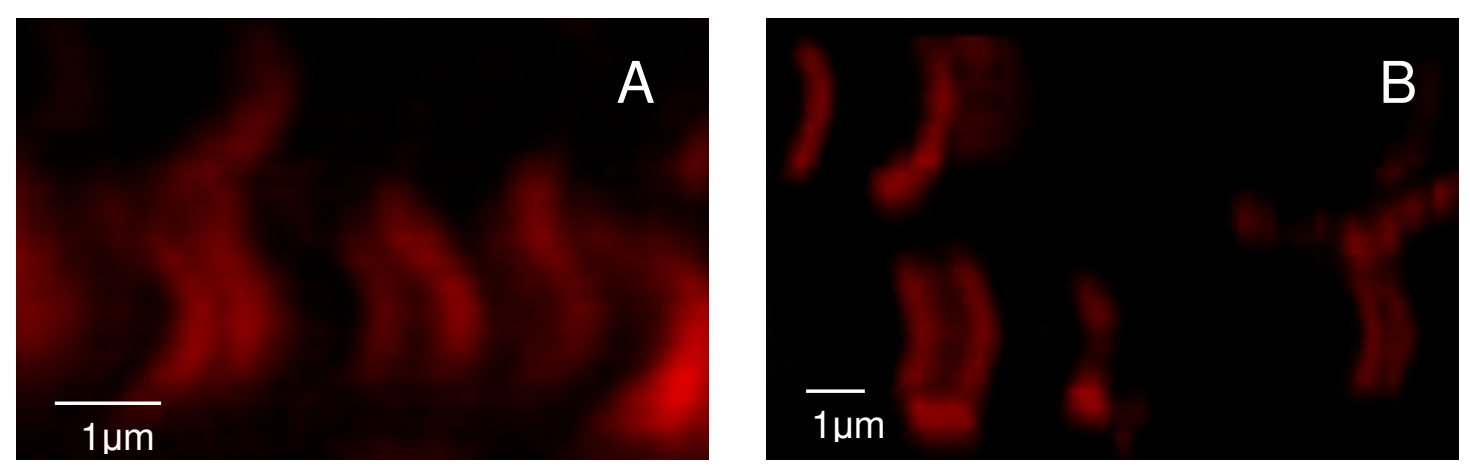

Figure 3.15. CLSM images showing longitudinal view of SCB hybridized with the eubacterial probe EUB338. A: Patiriella sp. B: Stichopus mollis.
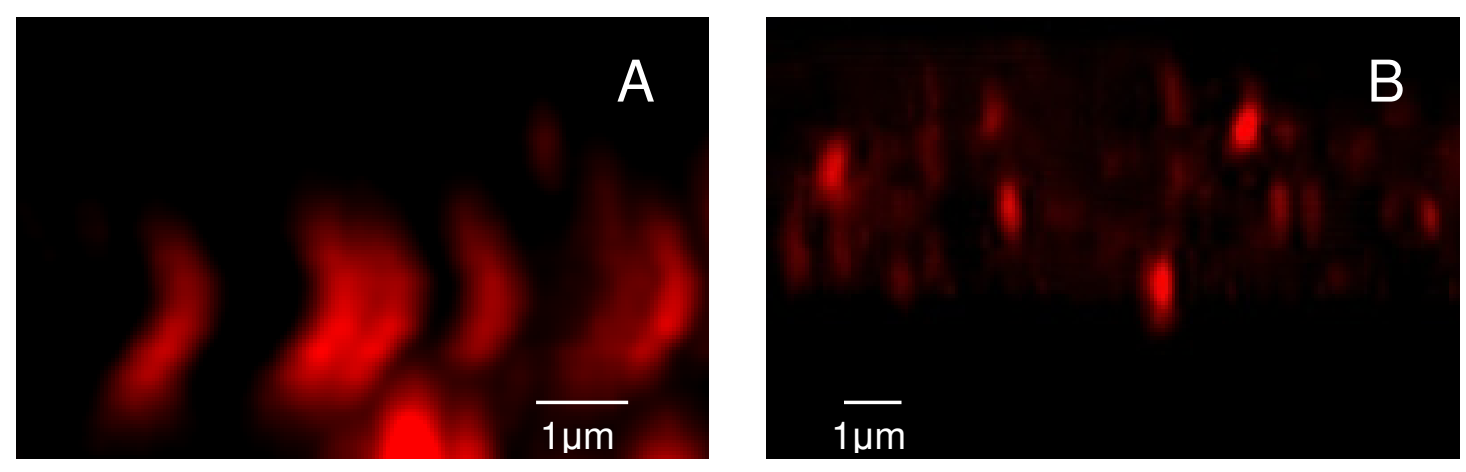

Figure 3.16. CLSM images showing longitudinal view of SCB from Stichopus mollis. A: SCB hybridized with the $\alpha$-proteobacteria specific probe ALF968. B: SCB hybridized with the $\gamma$-proteobacteria specific probe GAM42a. Note the apparent difference in morphology between the $\alpha$ - and $\gamma$-proteobacteria. The $\alpha-$ proteobacteria are larger and have a more uniform curved rod shape than the $\gamma$-proteobacteria.

As can be seen from Figures 3.15 - 3.16, SCB are bacilli varying in length from approximately 1 to $2 \mu \mathrm{m}$. Figure 3.16 shows the difference in size between the $\alpha$ - and $\gamma$ proteobacterial members of the SCB community of S. mollis. The $\alpha$-proteobacteria are noticeably larger, with a length of approximately $2 \mu \mathrm{m}$, compared to approximately $1 \mu \mathrm{m}$ for the $\gamma$-proteobacteria. 


\section{CARD-FISH}

The use of CARD-FISH did not increase the signal intensity from hybridized bacteria. Rather, it had the opposite effect, decreasing the bacterial signal relative to the autofluorescence of the echinoderm. The reason for this is assumed to be the choice of fluorescent dye: FITC is stimulated by the same wavelength of light/laser as the echinoderm autofluorescence; therefore the bacterial signal was drowned out by autofluorescence. Images of CARD-FISH results can be found in the appendix (Figures A11-12).

\subsection{Discussion}

The results obtained in this study suggest that the SCB communities of Patiriella sp. and S. mollis are made up almost entirely of proteobacteria. Patiriella sp. was found to contain $\alpha$-proteobacterial symbionts, while $S$. mollis contains both $\alpha$ - and $\gamma$-proteobacteria. The third echinoderm studied here, A. scabra was found to not contain SCB, in agreement with previous work (Kelly et al. 1994) (See also Chapter 2). There was no apparent difference in morphology between the $\alpha$-proteobacterial symbionts of the two host species; however the $\gamma$-proteobacterial symbionts of $S$. mollis differed in size and shape from the $\alpha$-proteobacterial symbionts. The prevalence of $\alpha$-proteobacteria in the SCB communities is interesting because $\alpha$-proteobacteria are very rarely found as extracellular symbionts (Burnett \& McKenzie 1997). Furthermore, the finding of abundant $\alpha$-proteobacteria in these species supports the results of earlier workers who found that the SCB of an ophiuroid belong to the $\alpha$-proteobacteria (Burnett \& McKenzie 
1997). Phylogenetic work carried out as part of this study found four bacteria which were identified as potential SCB of Patiriella sp. and S. mollis: two $\alpha$-proteobacteria, one $\gamma$ proteobacterium, and one which falls among both the $\alpha$ - and $\gamma$-proteobacteria (Chapter 2). These findings are partially supported by the results presented here. Clearly, $\alpha$ proteobacteria are present in high numbers in both species, which supports the finding of at least two putative $\alpha$-proteobacterial SCB through phylogenetic work. The fact that the $\gamma$-proteobacteria specific probe only hybridized in S. mollis samples is unexpected, as the $\gamma$-proteobacterium identified as a putative SCB through phylogenetic work was found both in Patiriella sp. and S. mollis. A possible explanation for this is that the SCB belonging to the $\gamma$-proteobacteria are much rarer than those belonging to the $\alpha$ proteobacteria (as was seen in S. mollis), and thus were not picked up by the GAM42a probe in the tissue sections analyzed here. This is not an unreasonable explanation, given that PCR is potentially a much more sensitive technique than FISH and can amplify DNA from as little as one cell. Moreover, the use of only two individuals of each echinoderm species for FISH assays may have affected the diversity of SCB detected. The $\gamma$ proteobacterium detected through sequencing and phylogenetic analysis was found in only one out of six individuals of both Patiriella sp. and S. mollis. This raises two possibilities: 1) The $\gamma$-proteobacterium is very rare, and in the majority of host individuals was not picked up by PCR due to the prevalence of other bacteria, and/or 2) The $\gamma$-proteobacterium varies in abundance between individuals, possibly to the point where it is not present at all in some individuals. Significant intraspecific variation in SCB abundance has been observed in ophiuroids (Foret \& Lawrence 2001), and there is no apparent reason why it should not also occur in the echinoderms studied here. Either 
of these two scenarios could account for the lack of successful hybridization in Patiriella sp. With greater sampling effort, SCB belonging to the $\gamma$-proteobacteria are likely to be found in Patiriella sp. using FISH.

The failure to hybridize the species-specific probes SCB1 and SCB2 is also rather curious, but there are several possible explanations for this result. The most obvious explanation would be that the bacteria targeted by these probes were not present in the echinoderm samples used for FISH. This is indeed a possibility, given that the two bacteria which these probes target were only found in one out of six individual echinoderms sampled for sequencing and phylogenetic analysis (Chapter 2). This does not necessarily mean that these two bacteria are not SCB; as mentioned above, intraspecific variation in SCB abundance does occur (Foret \& Lawrence 2001), and it may be the case that the bacteria targeted by these probes would be found by FISH if more echinoderm samples were examined. This may be particularly true for the probe SCB2, which was designed to target the $\gamma$-proteobacterium identified through sequencing and phylogenetic analysis. It is not surprising, given the reasons already put forward for the failure of GAM42a to hybridize in Patiriella sp., that this probe did not successfully hybridize. Even in S. mollis $\gamma$-proteobacteria were rare (around $20 \%$ of total SCB, see Table 3.5), so the chance of successful hybridization with the more specific SCB2 probe was always going to be quite slim.

Another possible explanation for the failure of these probes to hybridize is that they target a relatively inaccessible region of the $16 \mathrm{~S}$ rRNA molecule, and therefore could not bind 
to their target bacteria, even though the bacteria were present in the tissue sections. Higher order structure of the rRNA molecule can affect accessibility of certain target sites, and the problem is exacerbated by the highly conserved nature of much of the $16 \mathrm{~S}$ rRNA, which makes the design of specific probes targeting accessible regions difficult (Frischer et al. 1996, Fuchs et al. 2007). Figure 3.17 shows the relative accessibility of different regions of $16 \mathrm{~S}$ rRNA, and it can be seen from this that the probes SCB1 and SCB2 target a relatively inaccessible region. If this is indeed the reason behind the failure of probes SCB1 and SCB2 to hybridize, a potential solution would be the use of 'helper' probes. Helper probes are unlabelled oligonucleotides which target the same area as the labeled probe but are slightly longer and serve to open up the particular area of rRNA so the labeled probe can bind more easily (Fuchs et al. 2000). 


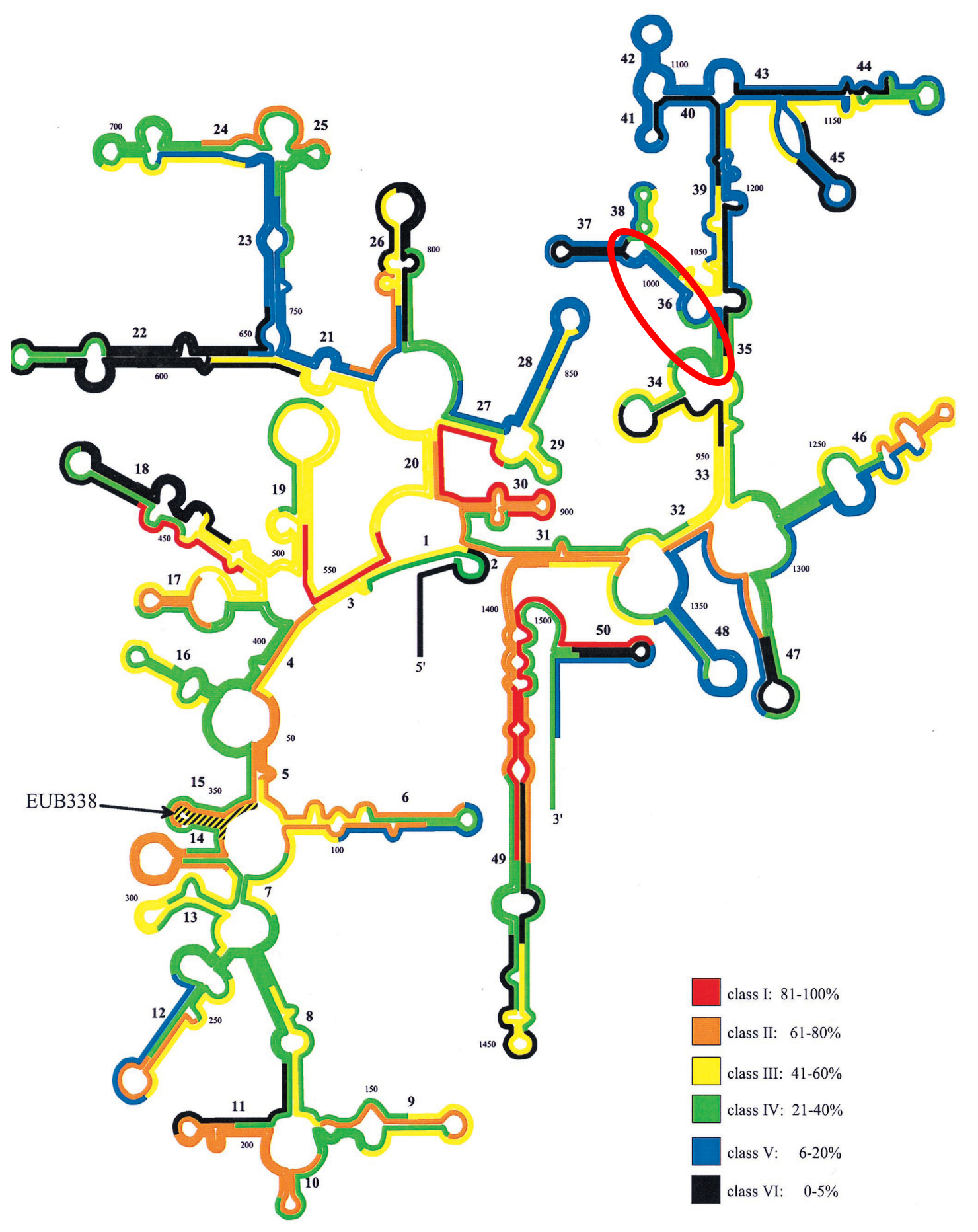

Figure 3.17. Diagram of $16 \mathrm{~S}$ rRNA, showing relative accessibility of different regions. Different colours represent relative fluorescence intensities of oligonucleotide probes bound to each region. The circled area is the approximate target region of the probes SCB1 and SCB2. The target region of the eubacterial probe EUB338 is also shown, indicating the relatively high accessibility to this probe. Figure reproduced from Fuchs et al. (1998). 
Finally, certain features of the target bacterial cells can play a role in the ability of a probe to hybridize effectively. These include low permeability of the cell wall and low ribosomal content (Fuchs et al. 2000). The former issue can be addressed through various permeabilization techniques such as pretreatment of tissue sections with lysozyme and proteinase $\mathrm{K}$, although this should not be necessary with gram-negative bacteria such as SCB, due to their relatively thin wall (Juretschko et al. 2004). The problem of low ribosomal content may be solved by using horseradish peroxidase (HRP) labeled FISH probes, as was done in the CARD-FISH assays of the present study. HRP-labeled probes act to increase the fluorescence signal through tyramide signal amplification (TSA - see Pernthaler et al, 2002 for more information), and are particularly useful when ribosomal number is low (Pernthaler et al. 2002). Due to the high cost of HRP-labeled probes, all other possible causes should be eliminated prior to considering this option.

While no attempt was made to accurately estimate the overall abundance of SCB in these host species, the fact that thousands of individual bacteria were counted in a fairly small number of samples used for estimates of community composition suggests that SCB abundance may be very high. Previous studies have found SCB loads of $10^{7}$ SCB cells $\mathrm{g}^{-1}$ ash free dry weight (Foret \& Lawrence 2001) to $10^{9} \mathrm{SCB}$ cells $\mathrm{g}^{-1}$ ash free dry weight (Kelly \& McKenzie 1995), and it would therefore not be surprising if SCB loads were also very high in the two symbiotic echinoderm species studied here.

The morphology of the SCB seen in Patiriella sp. and S. mollis is consistent with that of SCB from other echinoderms. SCB have been divided into three types based on their 
morphology (McKenzie \& Kelly 1994), and all SCB studied microscopically so far can be classified into one of these groups (Kelly et al. 1995, Kelly \& McKenzie 1995). The $\alpha$ proteobacteria observed in CLSM images in this study appear to be Type 2 SCB, which are described as curved or spiral-shaped, thin rods, with lengths of around 1-2 $\mu \mathrm{m}$ usually (McKenzie \& Kelly 1994, Kelly et al. 1995, Kelly \& McKenzie 1995). Type 2 SCB are the most commonly found type in all echinoderm classes (Kelly \& McKenzie 1995). The $\gamma$-proteobacteria observed here are harder to classify, but based on their relatively small size, they are most likely Type 1 or Type 2 SCB. Type 1 SCB are similar in size to Type $2 \mathrm{SCB}$, but are composed of two bacteria sharing an outer capsule (McKenzie \& Kelly 1994). The methods used here only show very general morphology, and thus the $\gamma$ proteobacteria cannot be assigned to either group with any certainty.

Observation of morphology using CLSM suffers from other limitations also. The need to make serial sections to produce three-dimensional images results in significant bleaching of the fluorescent signal, as the process takes some time. The problem of bleaching may be remedied by sectioning the echinoderms in different planes so as to remove the need for three-dimensional images, but if detailed morphological information is required the use of transmission electron microscopy (TEM) is recommended, as this has provided high quality images of SCB in previous studies (Holland \& Nealson 1978, Lesser \& Blakemore 1990, McKenzie \& Kelly 1994, Kelly et al. 1995, Kelly \& McKenzie 1995).

The bacteria identified as potential SCB of Patiriella sp. and S. mollis in Chapter 2 all fall within groups that have quite varied morphology. Therefore, the morphologies observed 
here cannot be taken as proof that the identification of these bacteria as SCB in Chapter 2 is correct. Certainly they do not refute the idea, as all of the taxa among which these species fall do contain at least some rod-shaped bacteria similar to those seen in Figures 3.15 and 3.16 (Krieg \& Holt 1984, Winn et al. 2006a).

\section{Conclusion}

Fluorescence in situ hybridization assays have shown that Patiriella sp. contains SCB belonging to the $\alpha$-proteobacteria, while $S$. mollis contains SCB belonging to the $\alpha$ - and $\gamma$-proteobacteria. It has also been shown that the proteobacteria make up nearly the entire SCB community in both species (approximately 95\% in Patiriella sp. and $85 \%$ in $S$. mollis). With further effort, it may in fact be revealed that all SCB in these two species belong to the proteobacteria, as the possibility exists that the small percentage of SCB not bound by proteobacterial probes represented members of the proteobacteria which simply failed to hybridize for some reason. Although the failure of the species-specific probes SCB1 and SCB2 to hybridize is disappointing, this study is nevertheless the first to demonstrate the phylogenetic affiliation of SCB in situ. Morphological assessment was limited by the methods used, but superficially the SCB seen here fit with previous descriptions from other echinoderms. Specifically, the SCB observed here appear to be “Type 2" SCB, which is the most commonly observed morphotype (Kelly \& McKenzie 1995). This raises the interesting question of whether the Type 2 SCB seen in other echinoderms also belong to the $\alpha$ - and $\gamma$-proteobacteria, or whether their common shape is coincidental or represents phenotypic plasticity in response to the environment inside the sub-cuticular space. 


\section{Future directions}

There are three main approaches that can be taken to improve and expand upon the results presented here.

First, more probes should be designed to target the bacteria identified as potential SCB in Chapter 2. Four bacteria were identified, and probes have already been designed for two of them. While these probes failed to hybridize in the present study, the use of unlabelled helper probes may enable their successful hybridization if attempted in future. If these two probes can be successfully hybridized, it leaves only two probes to be designed, which should be fairly straightforward based on the experience of designing the two existing probes. Even if all four probes have to be designed anew, it should not be too arduous, given enough time to evaluate the use of various helper probes and hybridization conditions.

Second, the study should be expanded to include individuals from different populations and sampled at different times of the year, as well as more species of echinoderms. By examining different populations, it should be possible to determine whether SCB community composition varies across time and space. This will be especially useful if the species-specific probes can be successfully hybridized. By determining if the same or similar bacteria are found in different echinoderm species, some light may be shed on the mode of transmission of the bacterial symbionts, and the potential co-evolution of host and symbiont. Highly similar bacterial assemblages have been found in distantly related 
species of other marine invertebrates such as sponges (Taylor et al. 2007), and a complex assemblage of microbes has been shown to be transmitted vertically (i.e. from parent to offspring) in the sponge Ircinia felix (Schmitt et al. 2007). It would be very interesting to know whether a similar mechanism is at work in the echinoderm-SCB symbiosis.

Finally, the mode of transmission can be further explored by sampling echinoderms at different stages in their life cycle. By performing FISH assays on samples from these different life stages (for example, egg, embryo, juvenile, adult), it should be possible to determine if vertical transmission is occurring, or, if vertical transmission does not occur, at what stage horizontal transmission occurs. 


\section{Chapter 4: Cultivation of sub-cuticular bacteria}

\section{Abstract}

The existence of symbiotic bacteria in the sub-cuticular space of echinoderms was first reported 30 years ago, and has since been shown to be a very common symbiosis, with more than half of all echinoderm species studied so far containing these sub-cuticular bacteria (SCB). In most echinoderms, the identity and function of SCB are unknown. If we are to fully understand the function of SCB, the ability to isolate and cultivate them is essential. Unfortunately, attempts at culturing SCB have so far been largely unsuccessful. The present study builds upon earlier culturing attempts by modifying previously used isolation methods, and trying to cultivate SCB on a range of media. Two echinoderm species known to contain SCB, Patiriella sp. (class Asteroidea) and Stichopus mollis (class Holothuroidea), were sampled; the asymbiotic Astrostole scabra was used as a negative control. Bacterial isolation methods were similar to those of previous studies, but with less emphasis on sterilization of echinoderm surfaces in order to reduce the chance of harming the SCB. Media which have previously been used in attempts to culture SCB (Zobell's modified 2216E medium) and marine bacteria (Marine Agar 2216) were used, as well as media containing tissue homogenates from the echinoderms under study. Successful isolation and cultivation was assessed by sequencing part of the $16 \mathrm{~S}$ rRNA gene from bacterial colonies and comparing this to previously identified SCB. Ten species of bacteria were thus identified. Seven of these were obtained from seawater samples. The remaining three, which belong to the genera Alteromonas, Shewanella and Psychrobacter, were only obtained from echinoderm samples, suggesting they could be 
SCB. However, based on their close phylogenetic relationships to free-living marine bacteria, it seems more likely that these three bacteria are not symbionts. Despite the apparent failure to cultivate SCB, the information gained from the present study can be used to guide future cultivation attempts.

\subsection{Introduction}

Echinoderms are known to form symbioses with a range of different bacteria. These symbioses include gut-associated bacteria in sea urchins (class Echinoidea) (Temara et al. 1993, Brigmon \& de Ridder 1998, Da Silva et al. 2006, Meziti et al. 2007), and bacteria associated with pinnules of feather stars (class Crinoidea) (Holland et al. 1991). In many cases, these bacteria are assumed to play a role in the nutrition of the host through various means. Of all the echinoderm-bacteria symbioses, however, the relationship between echinoderms and bacteria living beneath their outer cuticle (sub-cuticular bacteria (SCB)) is the most common, and the most well studied. Unlike the aforementioned symbioses and many other symbioses involving marine invertebrates and bacteria, where a nutritional role is often assumed, the nature of the echinoderm-SCB symbiosis remains rather enigmatic. This is despite their abundance (SCB appear to be present in the majority of echinoderm species (Kelly et al. 1994, Kelly \& McKenzie 1995)) and knowledge of their existence for 30 years (Holland \& Nealson 1978). There are many factors contributing to this lack of knowledge. The lack of studies using molecular techniques (such as $16 \mathrm{~S}$ rRNA gene sequencing) means that the identity of SCB has only been established in one host species (Burnett \& McKenzie 1997). Studies of SCB have so far relied mainly on microscopy, which has several drawbacks: SCB can be missed using 
microscopic techniques, leading to an underestimation of abundance, and, as noted in the seminal work on the subject by Holland and Nealson (1978), there is a tendency for the echinoderm cuticle to dissolve during fixation, further skewing results. In addition to these issues, there has been an overall lack of studies of SCB - only around 25 papers have been published on the topic in the last 30 years. Finally, the apparent inability to culture SCB has hampered understanding of this symbiosis, and this is the issue addressed in the present study.

Isolating and culturing SCB is an important step in determining their function, as it allows detailed microscopic examination, and analysis of their physiology and metabolism. Understanding the biology of SCB in this manner, especially when coupled with in situ methods such as PCR, fluorescence in situ hybridization and conventional microscopy, may allow us to finally work out what role SCB play in the symbiosis. Furthermore, if SCB are found to have biotechnological potential, as has been the case with many other marine invertebrate-associated bacteria, then obtaining pure cultures of SCB is imperative, so that any bioactive compounds can be isolated.

Previous workers claim to have cultured SCB from the brittle star Amphipholis squamata (Lesser \& Blakemore 1990), but subsequent studies have cast doubt on this claim due to non-repeatability of the results (Kelly et al. 1995). In particular, it is claimed that the methods used to eliminate surface-associated bacteria would almost certainly have killed any SCB present, and therefore the cultured bacteria must represent contamination of culture media rather than actual SCB. In the present study, methods were developed 
based on the successes and failures of previous culturing attempts, in an effort to efficiently isolate and culture SCB from Patiriella sp. (class Asteroidea) and Stichopus mollis (class Holothuroidea) while avoiding significant contamination issues.

\subsection{Materials and methods}

\section{Bacterial isolation and growth conditions}

Eight individuals each of Patiriella sp. (class Asteroidea) and Stichopus mollis (class Holothuroidea) were collected from Island Bay, Wellington, New Zealand (latitude $41^{\circ} 20^{\prime} \mathrm{S}$, longitude $\left.174^{\circ} 46^{\prime} \mathrm{E}\right)$ on different days throughout summer and transferred directly to plastic buckets containing unfiltered seawater. These two species were chosen as previous work has shown them to contain SCB (Chapter 2; Kelly et al., 1995). Within two hours of collection, echinoderms were rinsed with $1 \mu \mathrm{m}$-filtered seawater (FSW), and then left in FSW overnight. The following day, echinoderms were rinsed with $70 \%$ ethanol for 5 seconds, and sterile artificial seawater (ASW) for 30 seconds, and tissue sections, approximately $1 \mathrm{~cm}^{2}$, were excised from their dorsal surfaces. Tissue sections were homogenized in a ground glass tissue grinder along with $5 \mathrm{ml}$ sterile ASW. The homogenates were diluted 1:10 and 1:100 with sterile ASW or left undiluted, and each dilution was plated onto four Petri dishes containing a particular growth medium. Two plates of each dilution were incubated in a refrigerator at $4{ }^{\circ} \mathrm{C}$, and two at room temperature $\left(\sim 20^{\circ} \mathrm{C}\right)$. Sterile ASW and serial dilutions of FSW were also plated and incubated as above. Several plates were exposed to air only, and were incubated as above, serving as negative controls. All steps prior to incubation were carried out in a laminar 
flow hood. Figure 1 presents a graphical representation of the sampling and culturing process. Plates were incubated at the above temperatures for 48 hours, a time span which was found to allow sufficient growth of bacteria without the risk of overgrowth (data not shown).

\section{Culture media}

Throughout the course of these culturing experiments, four different culture media were used.

The first medium used was Marine Agar 2216 (Difco), prepared following the manufacturer's instructions.

After completing culturing attempts using this medium, a modified medium was made using Marine Agar 2216, mixed with homogenized Patiriella sp. or S. mollis tissue, depending on which echinoderm species was being studied. In order to make this medium, one sea cucumber (S. mollis) or two cushion stars (Patiriella sp.) were homogenized in $500 \mathrm{ml}$ sterile double-distilled water using a $1 \mathrm{~L}$ Waring laboratory blender. Marine Agar 2216 was then made, following the manufacturer's instructions, but substituting $\sim 500 \mathrm{ml}$ of water with homogenate.

The third medium used was a further variation on the above media. Echinoderms were homogenized as above, but $15 \mathrm{~g} \mathrm{l}^{-1}$ of Select Agar (Invitrogen) was used in place of Marine Agar 2216. Select Agar is a solidifying agent, containing no nutrients; hence the 
only nutrients present in this medium were those provided by the homogenized echinoderms.

The final medium used was Zobell modified 2216E medium (Zobell 1941). This medium was prepared according to the method of Lesser and Blakemore (1990), with the addition of $15 \mathrm{~g} \mathrm{l}^{-1}$ Select Agar as a solidifying agent. Constituents of Marine Agar 2216 and Zobell modified 2216E medium are listed in Appendix Tables A8 and A9, respectively. The recipe for ASW used in this study can be found in Appendix Table A10. All media were sterilized by autoclaving at $121{ }^{\circ} \mathrm{C}$ for 15 minutes, and approximately $20 \mathrm{ml}$ of medium was poured into each Petri dish. Petri dishes used were sterile 90 x $15 \mathrm{~mm}$ plastic dishes.

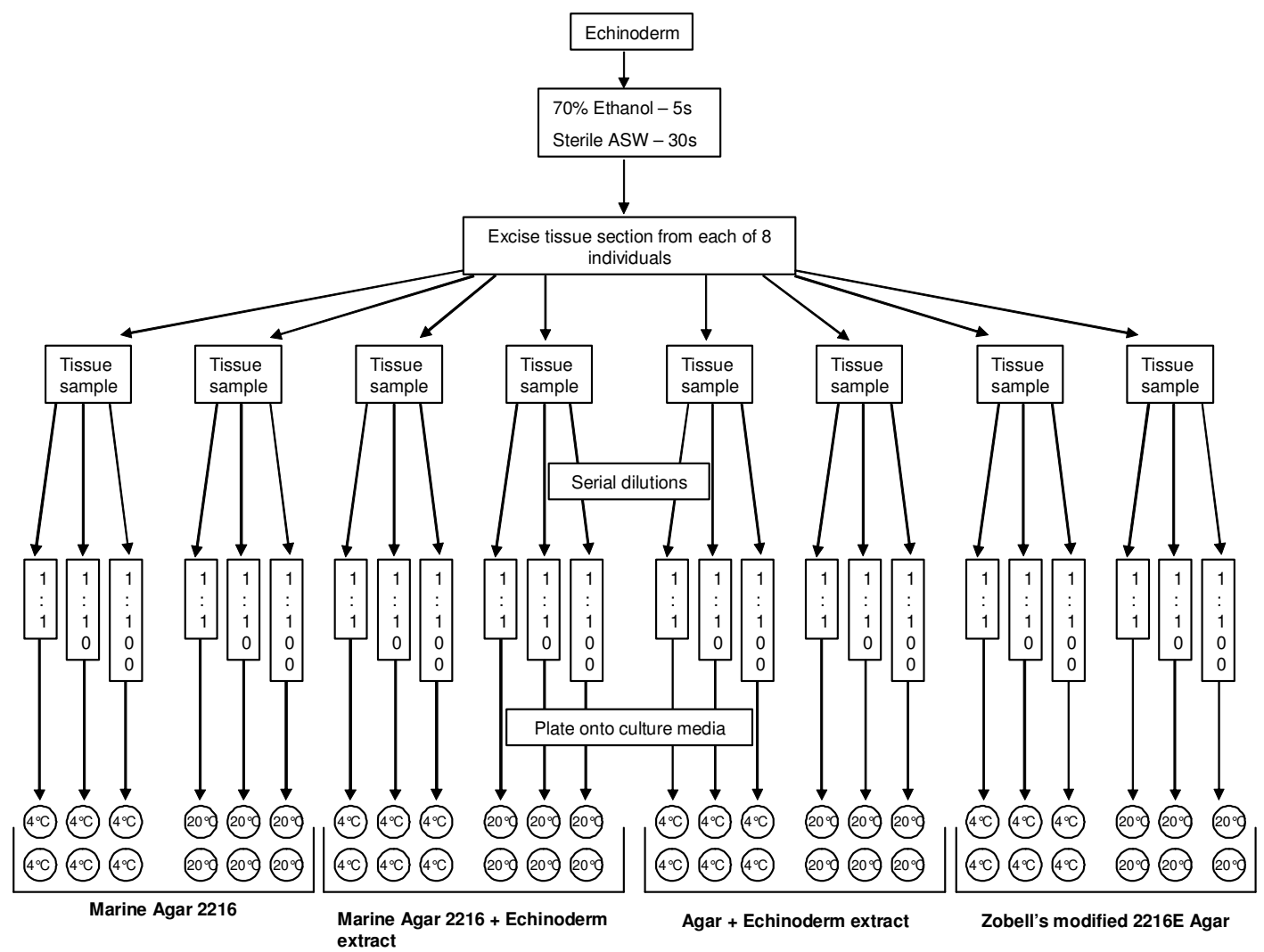

Figure 4.1. Diagram outlining the sampling and culturing process. 


\section{DNA extraction and PCR}

After incubation for 48 hours, one plate corresponding to each bacterial source (i.e. Patiriella sp., S. mollis, FSW and ASW) was selected from each of the different culture media. Selection was based on the presence of a sufficient number of well-spaced colonies. Up to fifteen colonies were picked from each plate for DNA extraction. Colonies were picked haphazardly, but when colonies were present which clearly differed in terms of colour or macroscopic appearance, care was taken to include at least one of each of these colonies. A $10 \mu$ l-micropipette equipped with a sterile $10 \mu \mathrm{l}$ tip was used to pick colonies and transfer them to $15 \mathrm{ml}$ screw-cap polypropylene tubes, containing $4 \mathrm{ml}$ culture medium for overnight growth. Culture media were prepared in the same manner as those already mentioned, except that Marine Broth 2216 (Difco) was used in place of Marine Agar 2216, and agar was not added to the other media. This resulted in four media for overnight growth, corresponding to the solid media used for initial growth: Marine Broth 2216, Marine Broth 2216 + echinoderm homogenate, echinoderm homogenate only and Zobell modified 2216E medium (Lesser \& Blakemore 1990). Colonies picked from plates containing Marine Agar 2216 + echinoderm homogenates were grown overnight in both standard Marine Broth 2216 and Marine Broth $2216+$ echinoderm homogenate in order to determine if the bacteria were relying on the echinoderm homogenate for growth. All other selected colonies were only grown overnight in the medium corresponding to that which they were plated on. After growing bacteria overnight, OD600 measurements were taken using a Helios Alpha spectrophotometer (Thermo Scientific) to determine growth. Optical density measurements were not relied upon solely, as the inherent turbidity of several of the 
media used, combined with the settlement of some of the echinoderm homogenates at the bottom of culture tubes meant that such measurements were often not particularly informative. Culture tubes were therefore checked by eye to determine generally whether growth had occurred. Prior to DNA extraction, $0.5 \mathrm{ml}$ of culture medium was transferred from each culture tube to a sterile $1.5 \mathrm{ml}$ screw-cap tube and mixed with $0.5 \mathrm{ml}$ of $80 \%$ glycerol, then placed into a $-80{ }^{\circ} \mathrm{C}$ freezer for long-term storage.

Where sufficient growth had occurred, bacterial DNA was extracted as follows: $1.5 \mathrm{ml}$ of medium was removed from each culture tube and transferred to a $1.5 \mathrm{ml}$ microcentrifuge tube. Bacterial cells were pelleted by centrifugation at $15,000 \times \mathrm{x}$ for 5 minutes, and supernatant was extracted using a $1 \mathrm{ml}$ micropipette and discarded. The bacterial pellet was resuspended in $400 \mu \mathrm{l}$ sterile TE buffer (10 mM Tris, $1 \mathrm{mM}$ EDTA, pH 8.0), lysozyme was added to a final concentration of $2 \mathrm{mg} \mathrm{ml}^{-1}$, and the solution was incubated at $37^{\circ} \mathrm{C}$ for 30 minutes. Sodium dodecyl sulfate (SDS) and proteinase K were then added to final concentrations of $1 \% \mathrm{w} / \mathrm{v}$ and $0.2 \mathrm{mg} \mathrm{ml}^{-1}$, respectively, and the suspension was incubated at $65^{\circ} \mathrm{C}$ for 15 minutes. Following incubation, $400 \mu \mathrm{l}$ TE-buffered phenol was added and mixed, the tube was centrifuged at $15,000 \times \mathrm{g}$ for 10 minutes, and the upper aqueous layer was transferred to a fresh microcentrifuge tube. $400 \mu$ chloroform was added to the new tube and mixed, the tube was centrifuged at $15,000 \times \mathrm{x}$ for 10 minutes and the upper aqueous layer was transferred to a fresh microcentrifuge tube. $1 \mathrm{ml}$ cold 99\% ethanol and $50 \mu 13 \mathrm{M}$ sodium acetate were added to the new tube, which was then centrifuged at $15,000 \times \mathrm{g}$ for 20 minutes. Supernatant was removed, $400 \mu \mathrm{l}$ cold $70 \%$ ethanol was added, and the tube was centrifuged again at $15,000 \times \mathrm{x}$ for 5 minutes. The 
supernatant was removed and the pellet was dried in a $37{ }^{\circ} \mathrm{C}$ heating block. After drying, the DNA pellet was resuspended in $100 \mu \mathrm{l}$ sterile double-distilled water and stored at 4 ${ }^{\circ} \mathrm{C}$.

Part of the $16 \mathrm{~S}$ rRNA gene of the extracted DNA was amplified using the previously published eubacterial forward primer PLK1 (Klaschik et al. 2002) in conjunction with the $\alpha$ - and $\beta$-proteobacteria specific reverse primer ABR1 and the $\gamma$ - and $\delta$-proteobacteria specific reverse primer GDR2. The primer sequences were as follows: PLK1, 5'-TAC GGG AGG CAG CAG T-3'; ABR1, 5'-CCA TGA GGA CTT GAC GTC-3'; GDR2, 5'TAG CAC GTG TGT AGC CCT-3'. PCR mixtures contained 1 U of BIOTAQ Taq polymerase (Bioline), $1 \times \mathrm{NH}_{4}$ reaction buffer, $1.5 \mathrm{mM} \mathrm{MgCl}_{2}, 0.2 \mathrm{mM}$ deoxynucleotide triphosphates (dntps), $0.8 \mu \mathrm{M}$ of forward primer, $0.4 \mu \mathrm{M}$ of each reverse primer and $1 \mu \mathrm{l}$ of undiluted template DNA in a final volume of $25 \mu$ l. Cycling conditions were as follows: initial denaturation at $94{ }^{\circ} \mathrm{C}$ for 5 minutes, 30 cycles of $94{ }^{\circ} \mathrm{C}$ for 1 minute, 60 ${ }^{\circ} \mathrm{C}$ for 1 minute, and $72{ }^{\circ} \mathrm{C}$ for 1 minute, and a final extension step of $72{ }^{\circ} \mathrm{C}$ for 5 minutes. PCR products were visualized using a $1.0 \%$ agarose gel stained with ethidium bromide and run in Tris-acetate-EDTA (TAE) buffer at $100 \mathrm{~V}$ for 1 hour. Molecular weight marker X (Roche) was used to estimate sequence length, and amplicons of the correct size were purified using a High Pure PCR Product Purification kit (Roche Applied Science). 


\section{Sequencing and phylogenetic analysis}

PCR products were digested at $37{ }^{\circ} \mathrm{C}$ overnight using $20 \mathrm{U}$ of $M s p \mathrm{I}$ (New England Biolabs), 1 x reaction buffer and $3.0 \mu \mathrm{L}$ PCR product in a total volume of $10 \mu \mathrm{L}$. Restriction digests were visualized using a 3.0\% agarose gel stained with ethidium bromide and run in $0.5 \mathrm{x}$ TAE buffer at $75 \mathrm{~V}$ for 1.5 hours. Fragment sizes were estimated by comparison with molecular weight marker X (Roche) and PCR products were grouped according to restriction fragment length polymorphism (RFLP) banding patterns. DNA was quantitated using a Nanodrop 1000 spectrophotometer (Thermo Scientific), and DNA was diluted with sterile, double-distilled water where necessary. Forward and reverse strands of one representative PCR product from each RFLP group were sequenced using a BigDye Terminator v3.1 cycle sequencing kit on an ABI3730 Genetic Analyzer (Applied Biosystems) at the Allan Wilson Centre for Molecular Ecology and Evolution, Massey University.

Sequences were compared to those in existing databases using the Basic Local Alignment Search Tool (BLAST) algorithm (Altschul et al. 1990) to identify similar sequences. All sequences were aligned using the Fast Aligner in the ARB software package (Ludwig et al. 2004). Sequences that had $>97 \%$ identity were clustered together using the online program FastGroupII (Yu et al. 2006). Maximum likelihood, maximum parsimony and neighbour-joining trees were obtained using ARB (Ludwig et al. 2004), and a consensus tree was constructed based on these initial trees. 


\subsection{Results}

\section{Bacterial isolation and culturing}

All culture media supported growth of bacteria isolated from echinoderm samples and FSW. Bacteria from autoclaved ASW grew on all media containing echinoderm homogenates, but never on Marine Agar 2216 (Difco) or Zobell modified 2216E medium. Bacteria were never seen to grow on negative control plates. Growth did not appear to be affected by incubation temperature; colonies formed on plates incubated at $4{ }^{\circ} \mathrm{C}$ almost as rapidly as those at $20^{\circ} \mathrm{C}$, and did not appear to differ in colour or morphology. For the purpose of overnight growth and subsequent DNA extraction, plates incubated at $4{ }^{\circ} \mathrm{C}$ were used. The different dilutions of echinoderm homogenates and FSW produced different densities of colonies. Plates which had a high number of colonies, while still being sufficiently spaced to isolate individual colonies, were chosen for colony removal and overnight growth in culture broth. Table 4.1 shows which plates colonies grew on, and the homogenate and seawater dilutions that were selected for overnight growth. 
Table 4.1. Results of culturing assays

\begin{tabular}{|c|c|c|c|c|c|c|}
\hline Sample & M.A. 2216 & M.A + S.M. & M.A. + P.S. & Agar + S.M. & Agar + P.R. & Zobell \\
\hline S. mollis & $1: 10$ & 1 & $\sim$ & 1 & $\sim$ & 1 \\
\hline Patiriella sp. & 1 & $\sim$ & 1 & $\sim$ & 1 & 1 \\
\hline Seawater & 1 & 1 & 1 & 1 & 1 & $1: 100$ \\
\hline ASW & - & 1 & 1 & 1 & 1 & - \\
\hline Neg. Control & - & - & - & - & - & - \\
\hline
\end{tabular}

Abbreviations as follows: M.A. $=$ Marine Agar, S.M. $=$ S. mollis homogenate, P.S. $=$ Patiriella $\mathrm{sp}$. homogenate, Zobell = Zobell modified 2216E medium. " $-"=$ No colonies observed, " $\sim=$ culturing not attempted, "1, 1:10, 1:100" = amount of dilution producing best-spaced colonies ( 1 = undiluted). Each number in the above table represents a plate from which colonies were selected for overnight growth.

Figures 4.1 - 4.6 show Petri dishes which were analyzed further, as listed in Table 4.1. As can be seen in these photographs, the highest numbers of colonies were obtained from seawater samples, as would be expected given the abundance of bacteria known to exist in seawater. Echinoderm samples tended to produce a much lower number of colonies, which might suggest that SCB were effectively isolated and surface sterilization techniques were successful in removing seawater-borne and surface associated bacteria. However, on culture media containing echinoderm homogenates, sterilized ASW tended to produce more colonies than echinoderm samples which were homogenized with the same ASW (Figures 4.2-4.5). 


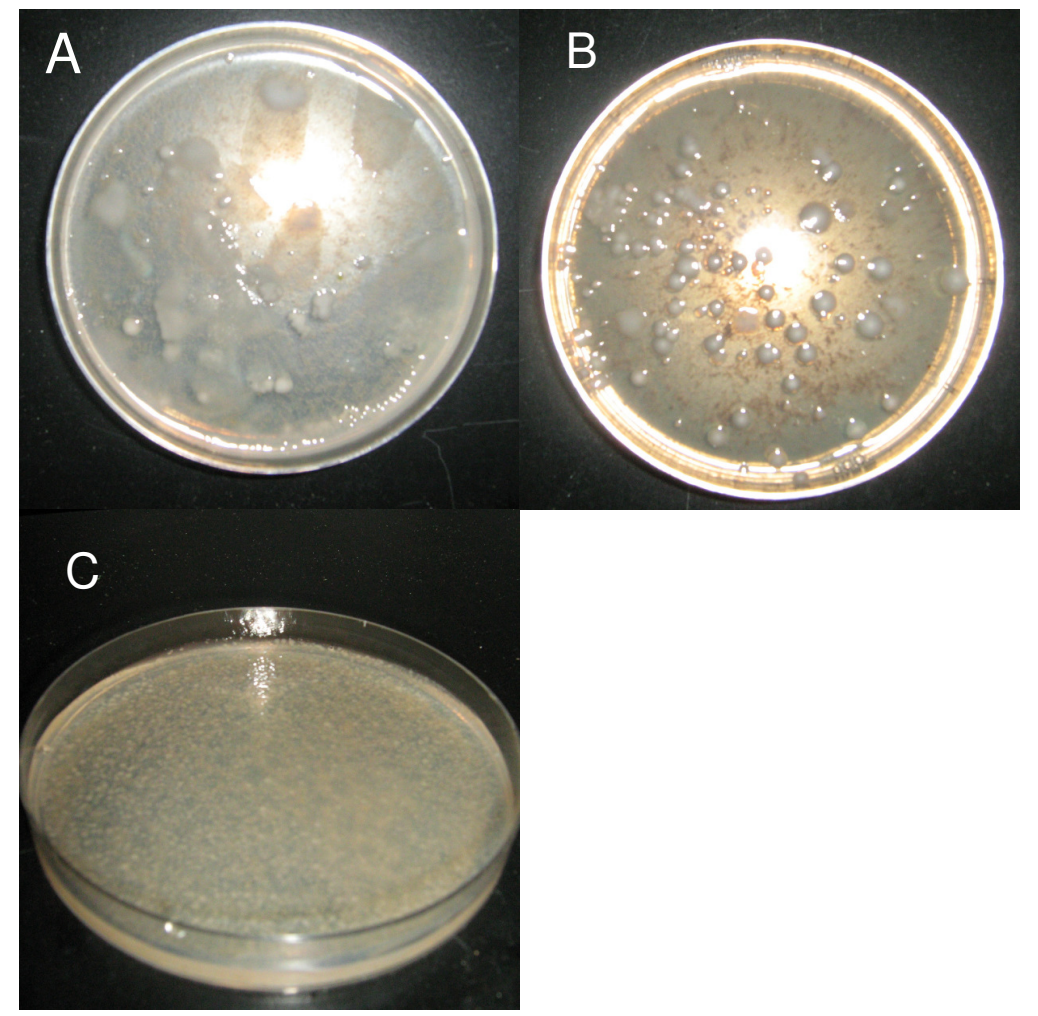

Figure 4.1. Petri dishes containing Marine Agar 2216, after 48 hours at $4{ }^{\circ} \mathrm{C}$. A: Patiriella sp. undiluted, B: S. mollis 1:10 dilution, C: $1 \mu \mathrm{m}$-filtered seawater, undiluted.

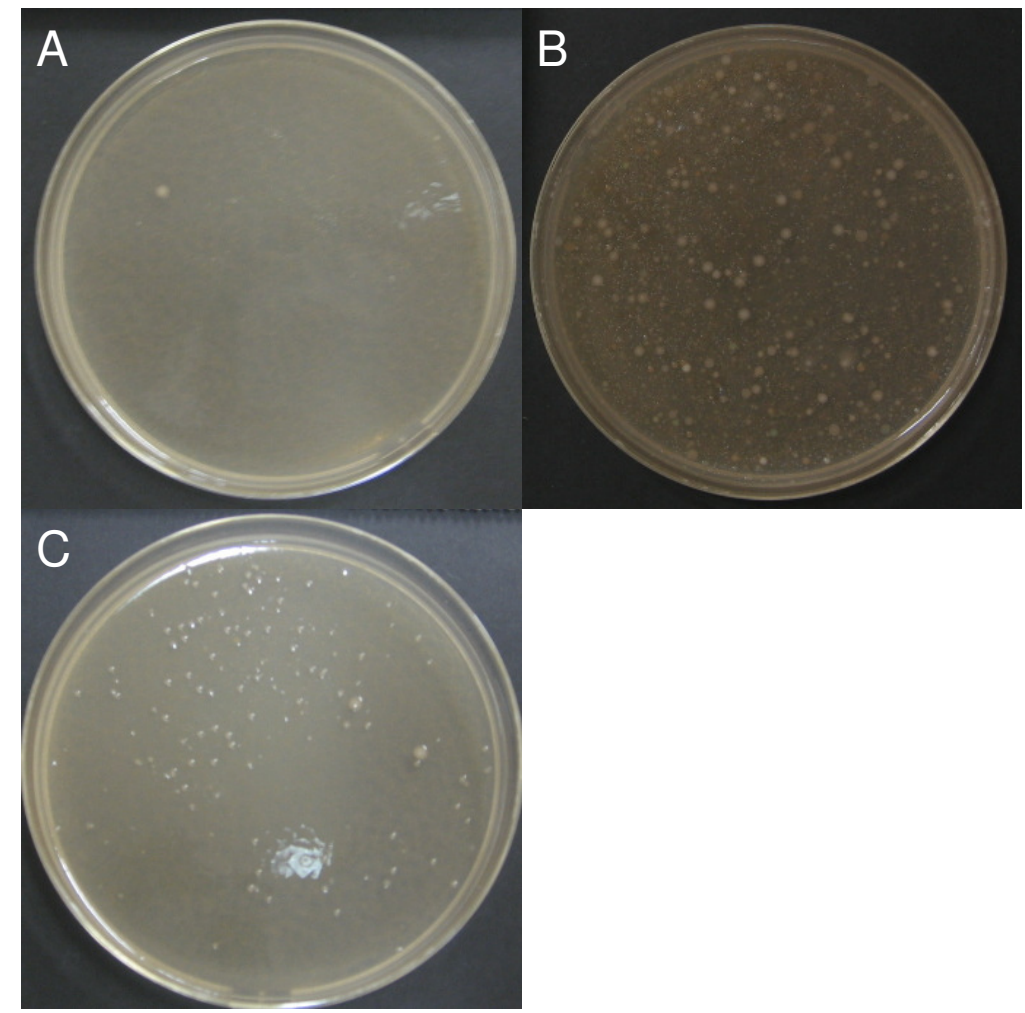

Figure 4.2. Petri dishes containing Marine Agar $2216+$ Patiriella sp. homogenate after 48 hours at $4{ }^{\circ} \mathrm{C}$. A: Patiriella sp., undiluted, B: $1 \mu \mathrm{m}$-filtered seawater, undiluted C: Sterilized ASW. 


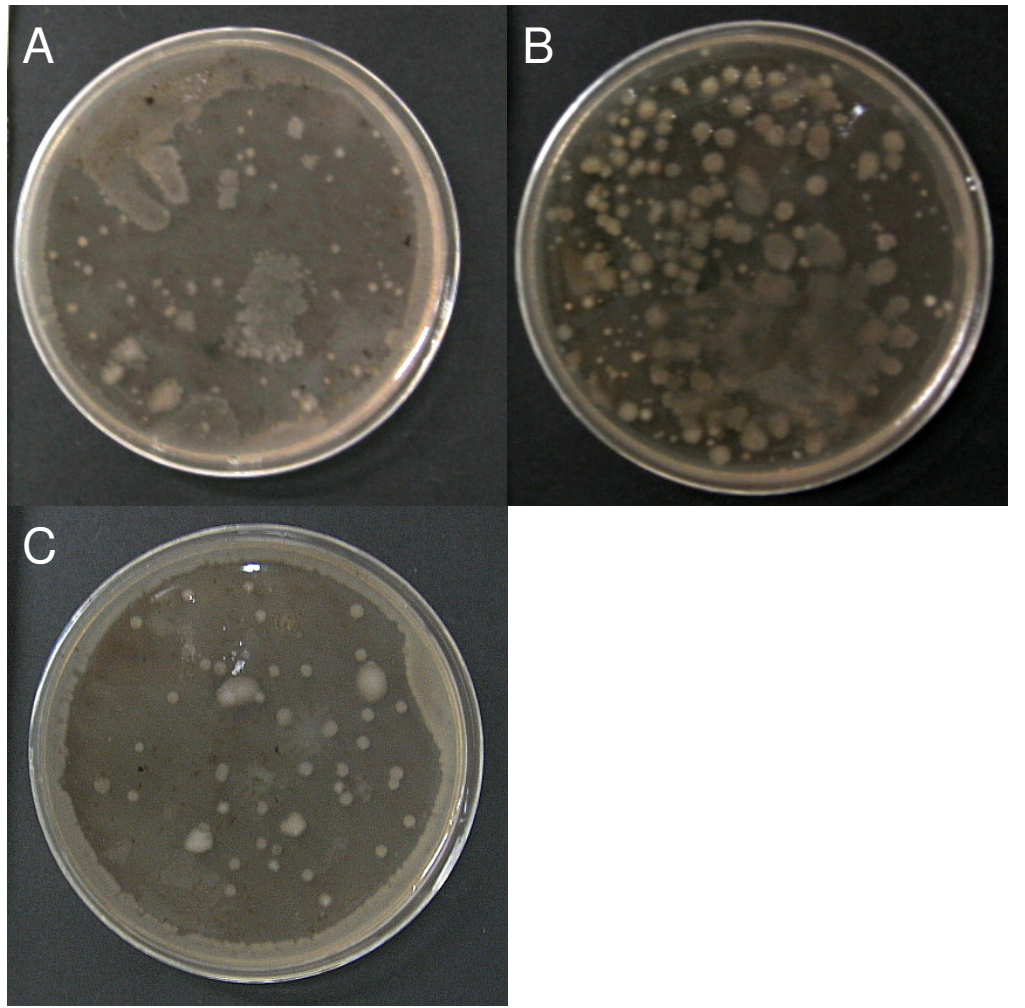

Figure 4.3. Petri dishes containing Marine Agar $2216+$ S. mollis homogenate after 48 hours at $4{ }^{\circ} \mathrm{C}$. A: $S$. mollis, undiluted, B: $1 \mu \mathrm{m}$-filtered seawater, undiluted, C: Sterilized ASW.

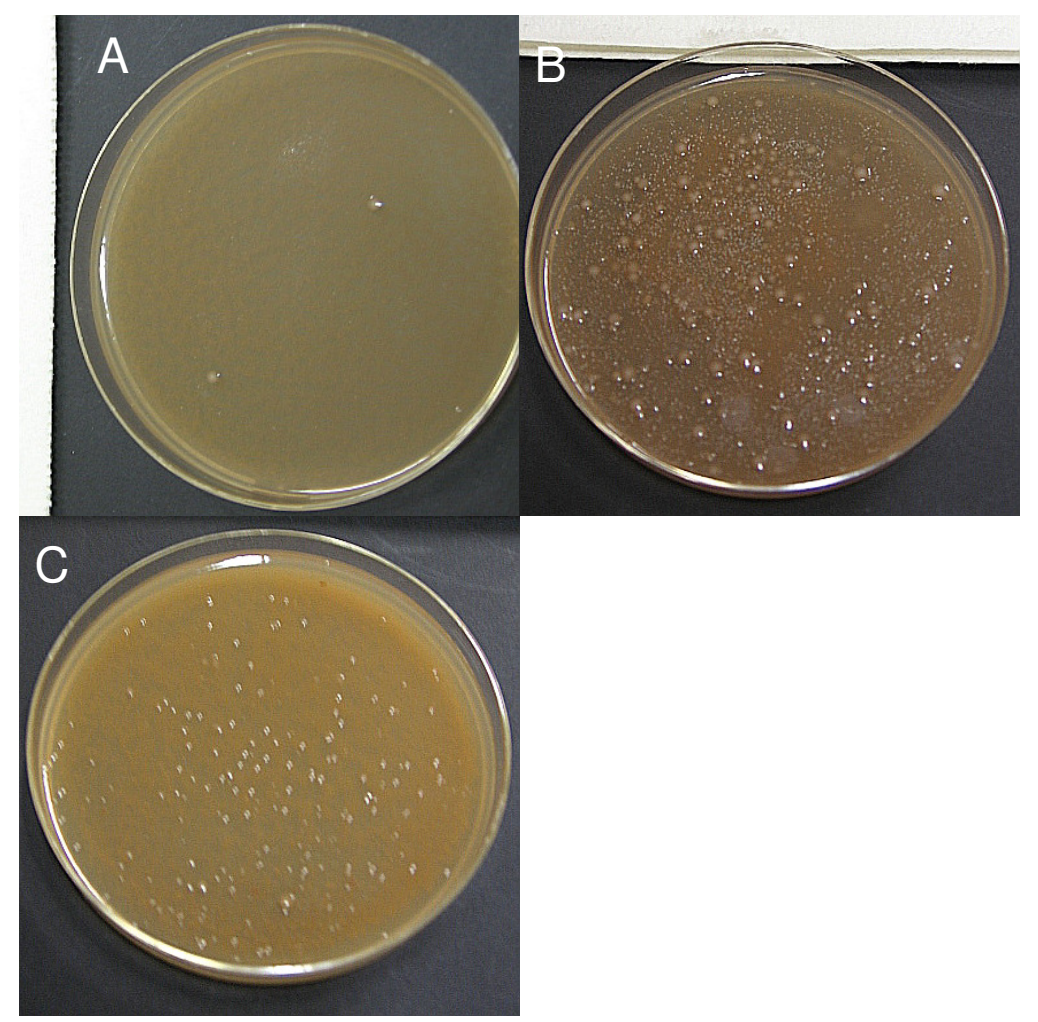

Figure 4.4. Petri dishes containing Agar + Patiriella sp. homogenate after 48 hours at $4{ }^{\circ} \mathrm{C}$. A: Patiriella sp., undiluted, B: $1 \mu \mathrm{m}$-filtered seawater, undiluted, C: Sterilized ASW. 

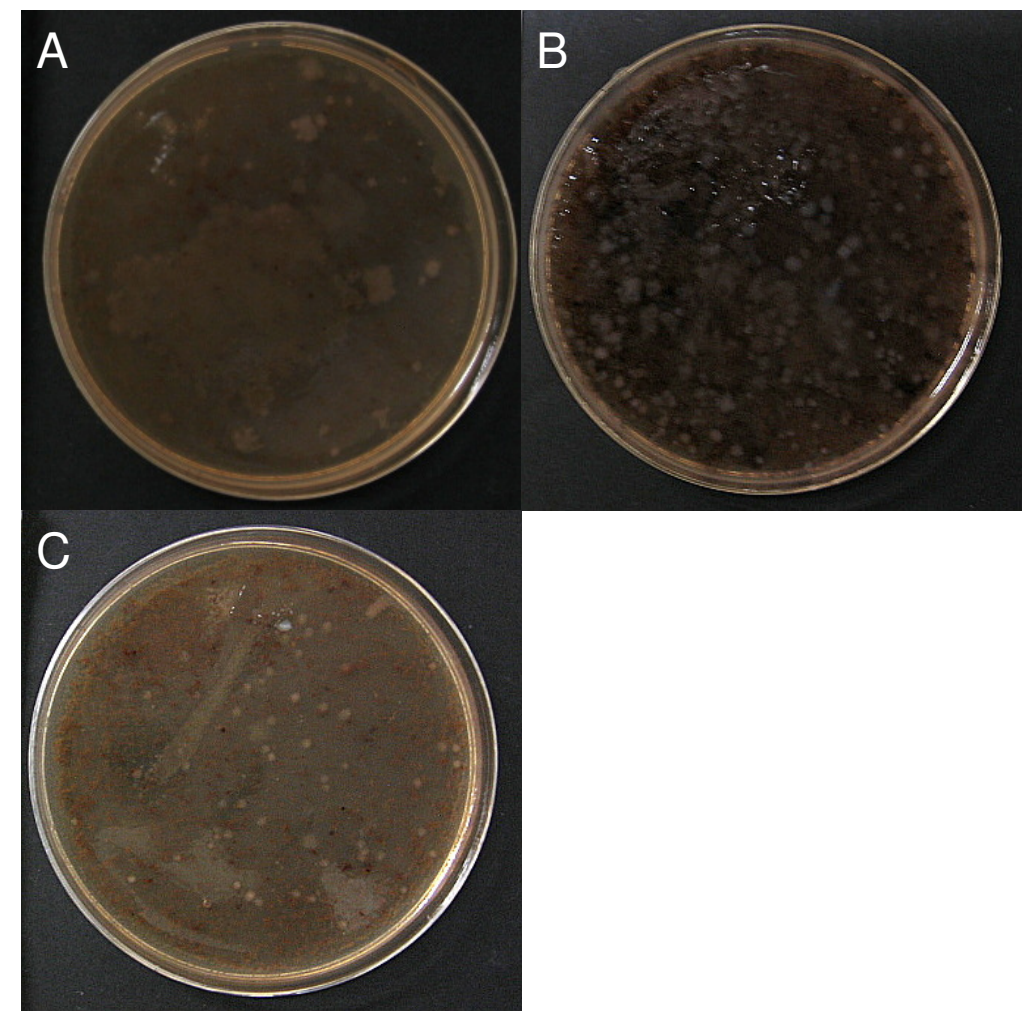

Figure 4.5. Petri dishes containing Agar + S. mollis homogenate after 48 hours at $4{ }^{\circ} \mathrm{C}$. A: S. mollis, undiluted, B: $1 \mu \mathrm{m}$-filtered seawater, undiluted, C: Sterilized ASW.

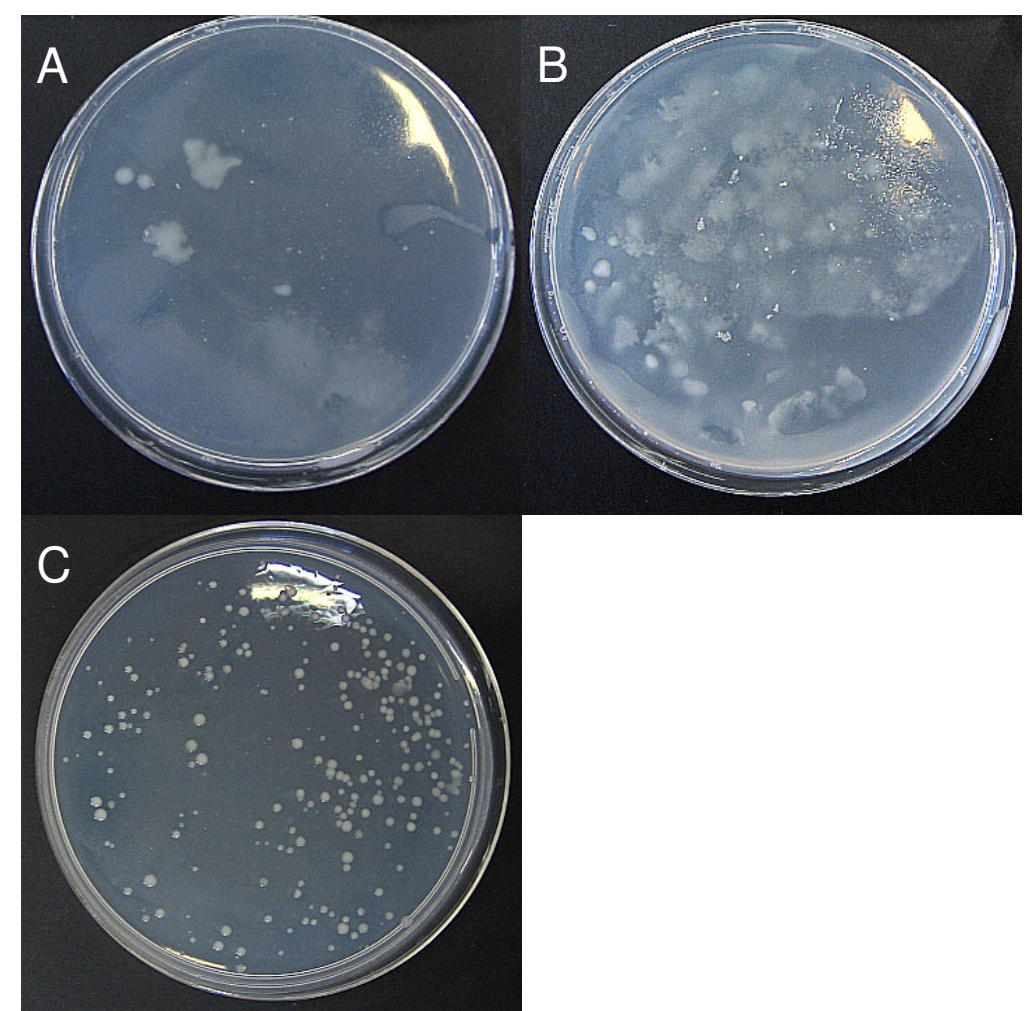

Figure 4.6. Petri dishes containing Zobell modified 2216E medium after 48 hours at $4{ }^{\circ} \mathrm{C}$. A: Patiriella sp., undiluted, B: S. mollis, undiluted, C: $1 \mu \mathrm{m}$-filtered seawater, 1:100 dilution. 


\section{DNA extraction and PCR}

DNA was successfully extracted from colonies grown overnight in Marine Broth 2216 and Zobell modified 2216E medium (picked from plates shown in Figures 1 and 6, respectively) and PCRs consistently showed bands of the correct size (approximately 850 b.p.) when run on agarose gels. A minority of colonies failed to produce PCR products, presumably because they were not proteobacteria and were therefore not amplified by the proteobacteria-specific PCR primers used. Colonies grown overnight in echinoderm homogenate only, failed to produce PCR products. It is possible that these colonies simply did not grow overnight, as the inherent turbidity of the echinoderm homogenate made it difficult to assess growth. Colonies picked from plates containing Marine Agar 2216 + echinoderm homogenate were grown overnight in standard Marine Broth 2216 as well as Marine Broth 2216 + echinoderm homogenate. Overnight growth was successful for all colonies in both media (as assessed by eye and spectrophotometric analysis). The ability of these colonies to grow in media with and without echinoderm homogenate shows that they were not reliant on anything in the homogenate for growth. For this reason, DNA extractions were not carried out on these bacteria, as they effectively represented additional standard Marine Agar 2216-derived colonies. Furthermore, the presence of a large number of colonies obtained from sterilized ASW (a negative control) on these media would have rendered any results obtained from these media somewhat questionable. 


\section{Sequencing and phylogenetic analysis}

The phylogeny of sequences obtained from cultured bacteria is presented in Figure 4.7. It appears from these results that none of the cultured bacteria are SCB. Of the ten species of bacteria cultured and sequenced, three were isolated only from seawater samples, and four were isolated from seawater and echinoderm samples, suggesting that they represent contaminating bacteria from the surrounding seawater rather than symbionts. The three remaining bacterial species may be $\mathrm{SCB}$, but are assumed to be non-symbiotic based on the identity of their nearest relatives, which are typically free-living. Going from top to bottom of Figure 4.7, these three bacteria appear to belong to the genera Alteromonas, Shewanella and Psychrobacter. 


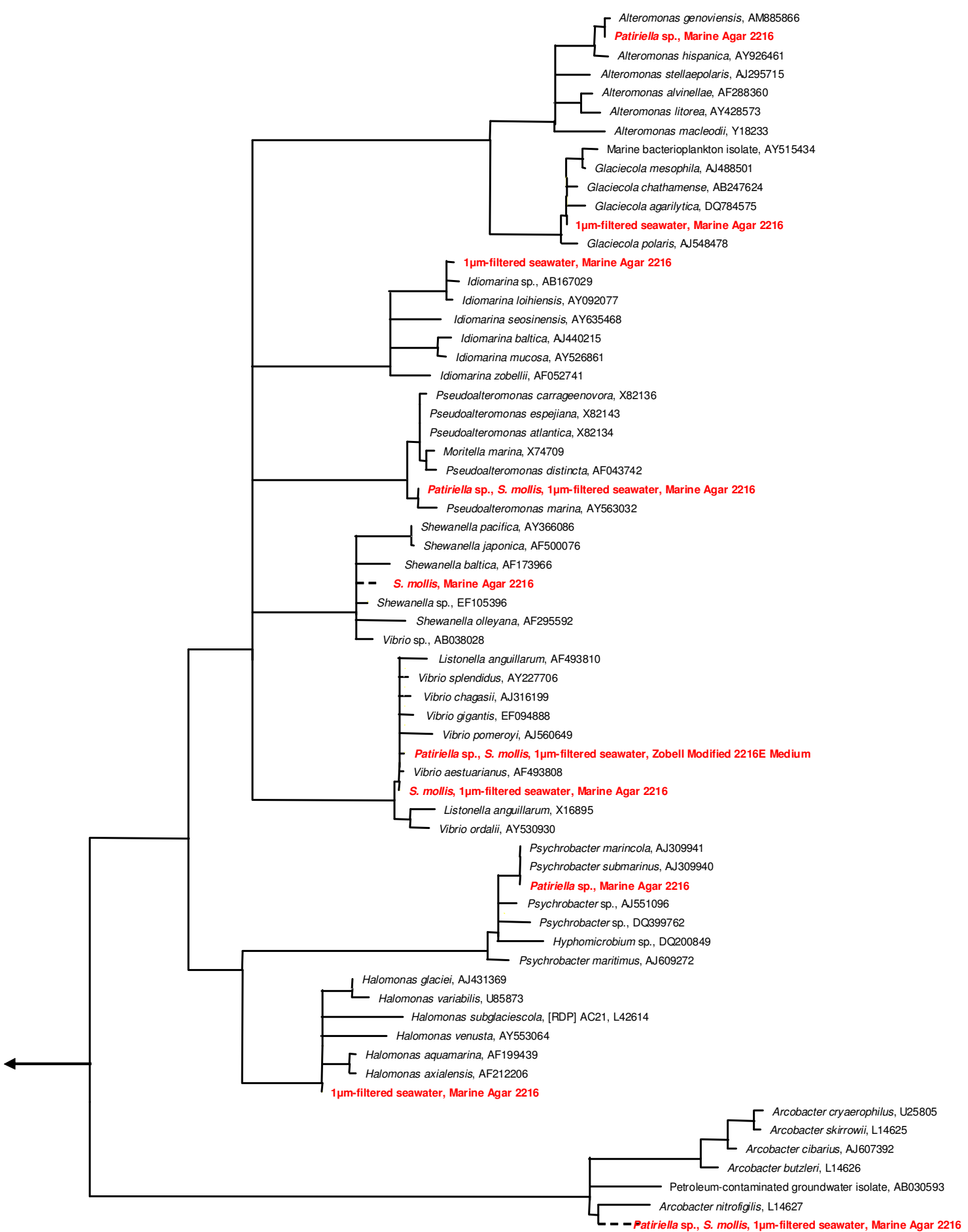

0.10

Figure 4.7. 16S rRNA-based phylogeny of cultured bacteria from echinoderm and seawater samples (shown in red). The displayed tree is a strict consensus tree based on maximum likelihood, maximum parsimony and neighbour-joining trees, constructed using long (>1,000 nucleotides) sequences. Shorter sequences (indicated by dashed lines) were added using the parsimony interactive tool in ARB. 


\subsection{Discussion}

\section{Sources of cultured bacteria}

In the present study, three different bacteria were identified which were found only in echinoderm samples. The first species was found in Patiriella sp., and belongs to the genus Alteromonas; the second was found in S. mollis, and belongs to the genus Shewanella; and the third species was found in Patiriella sp., and belongs to the genus Psychrobacter. These three genera contain few symbiotic species, and consist predominantly of free-living species (Baumann et al. 1984, Winn et al. 2006b). Therefore, it is possible that these bacteria are $\mathrm{SCB}$, but it is quite likely that they are contaminating bacteria associated with the echinoderm surface or seawater. The relationships of these three species to other bacteria are discussed below, along with the likely sources of these bacteria in the present study.

Species 1 (Alteromonas): Bacteria belonging to the genus Alteromonas resemble SCB morphologically (Baumann et al. 1984). Members of this genus are found almost exclusively in the marine environment (Baumann et al. 1984, Mikhailov et al. 2006). For the most part, these bacteria are free-living species, but there are a few examples of Alteromonas spp. forming symbioses with other organisms. Alteromonas spp. have been found in symbiosis with micro- (Stewart et al. 1997, Ashton et al. 2003) and macroalgae (Dimitrieva \& Dimitriev 1996), and the embryos of a crustacean (Gil-Turnes et al. 1989). In the cases of the macroalga and crustacean embryos, the bacteria were found to be associated with the outer surfaces of the organisms. If the bacterium isolated here is capable of forming similar symbioses, then it could indeed be a SCB (which are 
extracellular symbionts), or it could be associated (permanently or otherwise) with the surface of Patiriella sp. There is a high likelihood that this bacterium was in fact associated with the outer surface of the echinoderm, rather than the sub-cuticular space. The surface sterilization methods employed in the current study were intentionally quite gentle, as previous workers (Kelly \& McKenzie 1995, Strahl et al. 2002) have noted that surface sterilization methods used in early attempts to culture SCB (e.g. Lesser and Blakemore, 1990) were probably lethal to the SCB. There is therefore a chance that surface-associated bacteria survived the sterilization process and grew successfully on culture media.

The Alteromonas sp. found in symbiosis with crustacean embryos provides the host with protection against a pathogenic fungus (Gil-Turnes et al. 1989), and a similar role for SCB or surface-associated bacteria cannot be ruled out in echinoderms. The anti-fouling capability of echinoderms is well known (Bryan et al. 1996, McKenzie \& Grigolava 1996, Bavington et al. 2004, Guenther et al. 2007), and many bioactive compounds isolated from marine invertebrates have been found to be produced by associated bacteria (Jayatilake et al. 1996, Haygood et al. 1999, Dobretsov \& Qian 2004). Whether it is a SCB or a surface-associated bacterium, the possibility therefore exists that this species is in some sort of symbiotic relationship with Patiriella sp.

An alternative explanation for the presence of this bacterium in Patiriella sp. is that it is a contaminant from seawater. Although it was not found in seawater samples, it is not unreasonable to suggest that it might be with further sampling. This does not rule out the 
possibility of this species being a SCB, however, as the mode of transmission of SCB is largely unknown, and SCB may also exist as free-living forms in the ocean.

Species 2 (Shewanella): Members of the genus Shewanella are primarily aquatic bacteria, inhabiting a more diverse range of environments than bacteria belonging to the genus Alteromonas (Nealson \& Scott 2006, Hau \& Gralnick 2007). While it is possible that this bacterium is a SCB, this genus contains very few bacteria which have been identified as symbionts (Nealson \& Scott 2006). The one example of a known endosymbiosis involving a member of the genus Shewanella is that of $S$. pealeana, isolated from the nidamental gland of a squid (Leonardo et al. 1999). There are also several reports of epibiotic Shewanella spp., such as those isolated from intestines of marine fish and a bivalve mollusc by Satomi et al (Satomi et al. 2003). Interestingly, the squid symbiont $S$. pealeana is also found on the surface of its host's eggs, where it is thought to provide protection from predation or infection (Barbieri et al. 2001, Pichon et al. 2005). It is therefore conceivable that the bacterium identified here is a SCB or echinoderm surfaceassociated bacterium, and plays a similar role, aiding in antifouling of the echinoderm surface. However, the nearest phylogenetic matches to this species are all free-living bacteria. Therefore, it would not be surprising if this species was simply a seawater contaminant rather than a symbiont.

Species 3 (Psychrobacter): Psychrobacter is a genus whose members are commonly found in cold environments such as arctic and antarctic sediments, but also in more temperate marine environments and even in humans and other animals as opportunistic 
pathogens (Bozal et al. 2003, Bowman 2006). Of the three bacteria described here, this species is considered the least likely to represent a symbiont or epibiont of Patiriella sp. There are no reports of Psychrobacter spp. forming symbioses with other organisms; the only known interactions with eukaryotes are opportunistic infections of humans (Gini 1990, Guttigoli \& Zaman 2000), sheep (Vela et al. 2003), and fish (Hisar et al. 2002). Furthermore, the nearest relatives of the species identified are all free-living, having been isolated from either seawater or marine sediments. The close grouping of Hyphomicrobium sp. with this species is intriguing for two reasons. First, Hyphomicrobium belongs to the $\alpha$-proteobacteria, not the $\gamma$-proteobacteria as is the case with Psychrobacter. It appears that this is the result of a misdiagnosis - this Hyphomicrobium sp. should probably be relabeled as Psychrobacter sp. The second interesting aspect of this so-called Hyphomicrobium sp. is that it has been identified as a pathogen of the sea cucumber Apostichopus japonicus (class Holothuroidea). This raises the question of whether the species identified here could also be an echinoderm pathogen. While the nearest Psychrobacter spp. relatives are all free-living, this does not mean that they are not also capable of infection and pathogenesis. It is impossible to say, based on the evidence available, whether this species is a pathogen of Patiriella sp. or merely a contaminant from seawater or sediment, but both of these scenarios seem more plausible than a symbiotic origin.

Phylogenetically speaking, the three bacteria identified here do not fit with the results of previous work. Lesser and Blakemore (Lesser \& Blakemore 1990) suggest that the SCB of the brittle star Amphipholis squamata belongs to the genus Vibrio, Burnett and 
McKenzie (1997) found that the SCB of a the brittle star Ophiactis balli falls within the $\alpha$-proteobacteria, and the results obtained in Chapter 2 of the present study suggest that SCB belong to different genera to the three species listed above.

\section{Reasons for inconclusive results from cultivation}

It has become increasingly obvious in recent years that many bacteria are simply not cultivable by conventional means (Hugenholtz 2002). This may also be the case with SCB. However, very few attempts have been made to culture SCB, and, given the insights into their biology that culturing could yield, it seems only logical to at least try different culturing methods before giving up entirely. It seems likely that the bacteria cultivated in the present study are not $\mathrm{SCB}$, and in this section, possible reasons for the failure to cultivate SCB will be discussed briefly, and possible remedies or avenues of future research suggested. For this purpose, culturing methods will be separated into two components: bacterial isolation and culture media.

Bacterial isolation. As mentioned previously, the bacterial isolation method used in the present study (in particular the sterilization of the echinoderm surface) was considered relatively gentle compared to some earlier SCB culturing attempts. This does not mean, however, that this method did not result in the death of some or all SCB. The thin, permeable nature of the echinoderm cuticle means that even a very short exposure to ethanol could be lethal to SCB. If this was the case, it would be an obvious explanation for the failure to culture SCB. Given the limited timeframe available to complete the culturing experiments in the present study, a middle-ground was chosen in order to 
eliminate as many surface-associated bacteria as possible without harming the SCB. Whether or not this was achieved has not been empirically assessed, but the presence of what may be surface-associated bacteria on culture plates suggests that the method used was sufficiently gentle to prevent damage to the SCB. Ideally, a series of different isolation methods should be used in order to see which one results in successful cultivation of SCB with minimal contamination from seawater or surface-associated bacteria (assuming, of course, that culture media and growth conditions are optimal for SCB). No study to date has carried out a comprehensive set of SCB isolation experiments, but the closest comes from the work of Strahl et al (2002), and is shown in Figure 4.8. As can be seen in this diagram, a 30 second rinse in $70 \%$ ethanol (Regimen 2) was lethal to all bacteria. Bacteria were successfully isolated using the three other regimens, but it should be noted that the authors themselves admitted that they could not distinguish SCB from other bacteria associated with the brittle star host. It is obviously difficult to identify the true sources of cultured bacteria, as was found to be the case in the present study, but future studies, by combining the use of a wide range of isolation methods with the knowledge gained from in situ studies of SCB (using techniques such as PCR and fluorescence in situ hybridization), should be able to efficiently isolate, culture and identify SCB from a range of echinoderms. 


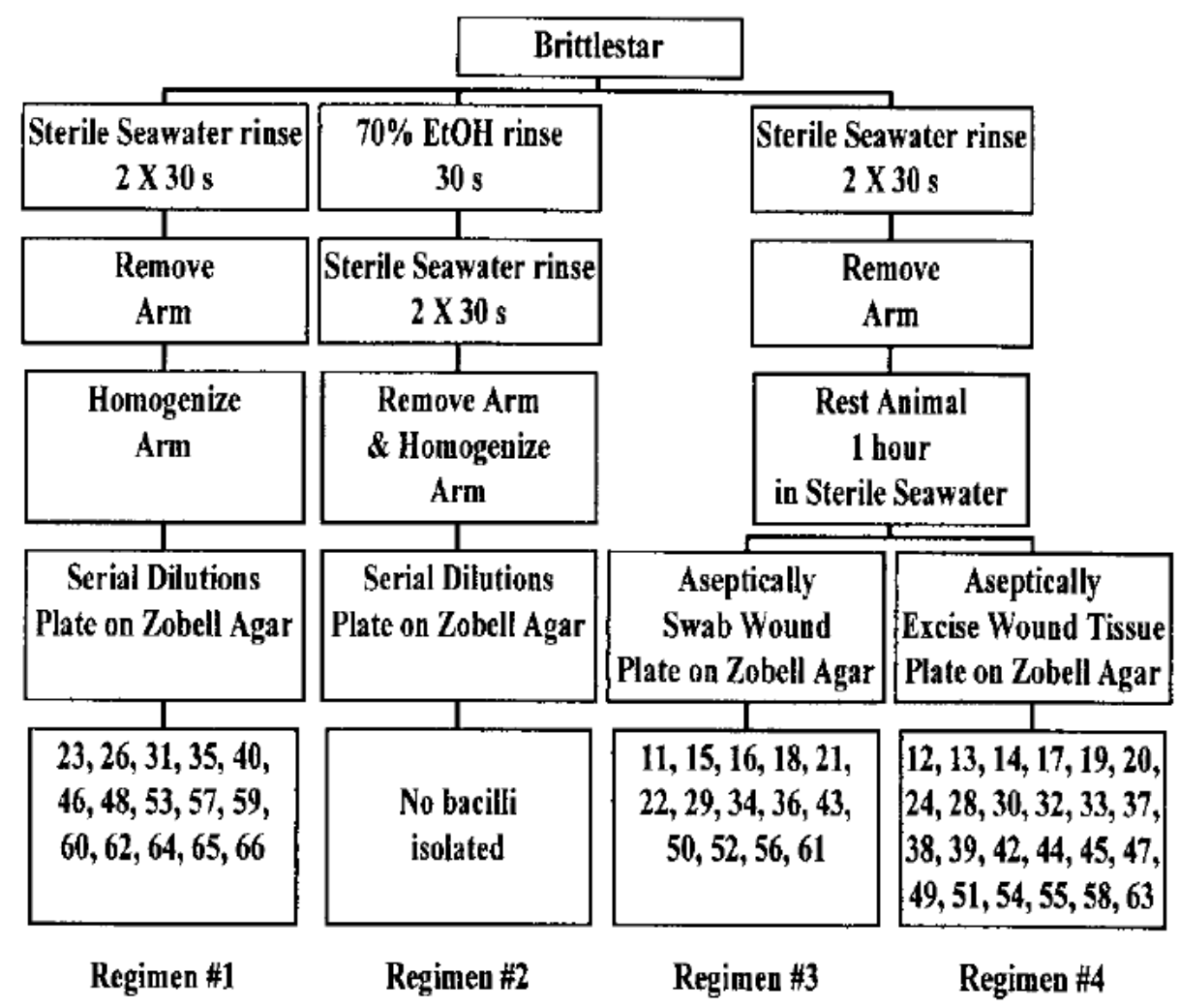

Figure 4.8. Diagram outlining SCB isolation methods used by Strahl et al (2002). Each number in the lower boxes represents a different bacterial isolate. Figure reproduced from Strahl et al (2002).

Culture media. Selecting the appropriate culture media is equally as important as efficient isolation of SCB. In the present study, several media were used which were selected based on those used in previous attempts to culture SCB (Zobell modified 2216E medium), or other marine bacteria (Marine Agar 2216). Additionally, Marine Agar 2216 was modified by the addition of host echinoderm homogenates. All of the media used were of necessity general media (i.e. non-selective), as the precise identity of SCB found in Patiriella sp. and S. mollis has not yet been established. General media such as these are useful in cases where the identity of the targeted bacteria is unknown, or where the aim is to culture a broad spectrum of bacteria from an environmental sample. However, there are drawbacks to the use of such media. The first, and most obvious potential 
problem is that the culture media may not provide the correct nutrients or energy source for SCB. The other major problem is that, due to the wide range of bacteria that are cultivable on such media, SCB may simply be out competed by other bacteria, thus decreasing the chance of selecting an SCB colony for analysis. This second issue is likely to be of particular concern in studies such as the present one, where the marine habitat of the host organisms may result in the presence of highly abundant bacteria in seawater which can potentially contaminate samples. If the second issue (competition from other bacteria) is in fact what occurred in the present study, a potential solution would be to analyze more colonies. While there is no guarantee this would result in the identification of cultured SCB, it would certainly increase the chance of doing so. However, even if SCB can grow on a particular general medium, they may be out-competed to the point that they fail to produce visible colonies before the faster-growing bacteria take over.

The most effective way of culturing SCB is likely through the use of selective media, using knowledge from in situ studies to guide the medium selection. Unfortunately, in the present study, results of sequencing and phylogenetic analysis of SCB were not available in time to be used in the selection of media, and general media were therefore used. Based on the findings of the present study (See Chapter 2), and previous work (Burnett \& McKenzie 1997) several media can be recommended for future attempts at culturing SCB. SCB for which phylogenetic relationships have been established are listed in Table 4.2 along with suggested selective culture media, and the bacterial groups targeted by each medium. In some cases, selective media are not available for a particular group, such as the Rhodobacteraceae (Table 4.2). In such cases, general media such as Marine Agar 
2216 or Zobell modified 2216E medium will have to be used. For other bacteria, such as the SCB which falls among the orders Legionellales, Rickettsiales and Thiotrichales (Table 4.2), the suggested media may not provide sufficient coverage of the target group to guarantee SCB will grow successfully. In these cases more general media may again have to be used, but the suggested media at least provide a starting point for future culturing experiments. 
Table 4.2. Suggested culture media for the growth of SCB identified in three echinoderm species.

\begin{tabular}{|c|c|c|c|}
\hline Host & SCB Family or Order* & Suggested media & Media specificity** \\
\hline \multirow{3}{*}{$\begin{array}{l}\text { Patiriella sp. } \\
\text { (Chapter 2) }\end{array}$} & Order Chromatiales & $\begin{array}{c}\text { Pfennig's Medium I, } \\
\text { modified for Purple Sulfur } \\
\text { Bacteria }\end{array}$ & $\begin{array}{l}\text { Order Chromatiales } \\
\text { (purple sulfur } \\
\text { bacteria) }\end{array}$ \\
\hline & \multirow{2}{*}{$\begin{array}{l}\text { Orders Legionellales / } \\
\text { Rickettsiales / } \\
\text { Thiotrichales }\end{array}$} & Legionella selective agar & Legionella spp. \\
\hline & & Blood glucose cystine agar & Francisella spp. \\
\hline \multirow{7}{*}{$\begin{array}{l}\text { S. mollis } \\
\text { (Chapter 2) }\end{array}$} & Order Chromatiales & $\begin{array}{c}\text { Pfennig's Medium I, } \\
\text { modified for Purple Sulfur } \\
\text { Bacteria }\end{array}$ & $\begin{array}{l}\text { Order Chromatiales } \\
\text { (purple sulfur } \\
\text { bacteria) }\end{array}$ \\
\hline & \multirow{3}{*}{$\begin{array}{c}\text { Order Rhizobiales } \\
\text { (Family Rhizobiaceae) }\end{array}$} & Rhizobium Medium 1 & Family Rhizobiaceae \\
\hline & & Rhizobium Medium 2 & Family Rhizobiaceae \\
\hline & & Yeast extract mannitol agar & Family Rhizobiaceae \\
\hline & $\begin{array}{l}\text { Order Rhodobacterales } \\
\qquad \text { (Family } \\
\text { Rhodobacteraceae) }\end{array}$ & $\begin{array}{l}\text { Marine Agar } 2216 \text { / Zobell } \\
\text { modified 2216E medium } \\
\text { (no selective media } \\
\text { available) }\end{array}$ & Marine bacteria \\
\hline & $\begin{array}{c}\text { Orders Legionellales / } \\
\text { Rickettsiales / }\end{array}$ & Legionella selective agar & Legionella spp. \\
\hline & & Blood glucose cystine agar & Francisella spp. \\
\hline & Order Rhizobiales & Rhizobium Medium 1 & Family Rhizobiaceae \\
\hline (Burnett \& & (Family Rhizobiaceae) & Rhizobium Medium 2 & Family Rhizobiaceae \\
\hline McKenzie 1997) & & Yeast extract mannitol agar & Family Rhizobiaceae \\
\hline
\end{tabular}

$*$ = taxonomic groups in which the identified SCB fall. $* *=$ Bacterial groups which are cultivable on the corresponding medium, according to Atlas (2005)(Atlas 2005). Note: As preparation of several of these media is somewhat complicated, preparation instructions are not listed here. Instead, the reader is referred to Atlas (2005). 
In addition to the selective media suggested in Table 4.2 , there is a range of media available which are selective on the basis of bacterial metabolism and nutrition (See Atlas (2005) for a wide range of culture media). If a particular symbiotic role has been ascribed to a certain $\mathrm{SCB}$, the possibility exists to test this theory, and in turn culture the SCB in question, by using selective media such as these. As an example, let us consider the SCB found in the brittle star Ophiactis balli by Burnett and McKenzie (1997). This SCB was found to be closely related to members of the rhizobacteria, and as such a role in nitrogen metabolism was suggested for this bacterium. If one wanted to test this theory, a culture medium specific for nitrogen-fixing bacteria could be utilized. This would have the dual effect of 1) potentially culturing the SCB of this species, and 2) determining that this bacterium is in fact involved in nitrogen fixation. Obviously, in many cases it is much more difficult to assign potential roles to $\mathrm{SCB}$, but nevertheless this remains a viable option for future attempts at culturing some SCB.

\section{Concluding remarks}

In the present study, cultivation of SCB from Patiriella sp. and S. mollis was not conclusively achieved. If none of the bacteria were indeed SCB, possible explanations for this are that the isolation method used resulted in death of the SCB, or that the culture media used were inappropriate for the bacteria under study. It has been shown previously that almost any bacterium can be cultured, given enough time and effort (Fry 2004). The empirical assessment of various isolation methods and culture media required for successful culture of SCB will obviously be time-consuming, but in the long run it will be worth the effort. As noted by Fry (2004), the only way of fully understanding 
biogeochemical cycles is through culturing bacteria of interest and studying their physiology. Moreover, if some of the hypothesized functions of SCB, such as production of antimicrobial compounds, are correct, successful cultivation of SCB becomes even more important. An interesting observation from the present study was that, in cases where bacterial colonies grew from ASW samples, more colonies were obtained from ASW alone than from echinoderm samples homogenized with ASW. A possible explanation for this is that the echinoderm homogenate somehow suppresses bacterial growth. If true, this may be attributable to the hypothesized antimicrobial action of SCB. One need only look at the range of bioactive compounds already isolated from marine invertebrate-associated microbes (See Hale et al. (2002) and Taylor et al. (2007) for reviews of natural products from bryozoan- and sponge-associated bacteria, respectively) to see the value in determining whether or not SCB produce similar compounds. If products with biotechnology applications can potentially be isolated from SCB, then, despite the difficulty, it is clearly worth the endeavour of culturing these bacteria. 


\section{Chapter 5: General discussion}

\subsection{Summary of results}

In this thesis, three species of echinoderm were examined in detail, in order to shed light on the possible symbiosis between them and sub-cuticular bacteria (SCB). The three species were Patiriella sp. (class Asteroidea), Stichopus mollis (class Holothuroidea) and Astrostole scabra (class Asteroidea). In addition to these three focal species, preliminary investigations were also carried out on Rosaster mimicus, Psilaster acuminatus, Perissasterias monocantha and Diplopteraster sp. (all class Asteroidea), by analyzing DNA sequences obtained from associated bacteria. With regard to the three main echinoderm species, the aims of this thesis were threefold:

1. To determine whether or not SCB are present, through the use of $16 \mathrm{~S}$ rDNA sequencing and, where present, to phylogenetically identify the SCB as accurately as possible.

2. To use fluorescence in situ hybridization (FISH) to provide further evidence for the presence or absence of SCB in each echinoderm species and to estimate the proportion of their SCB communities made up of different bacterial taxa.

3. To cultivate SCB from those species of echinoderm which were found to contain them. 
The results of FISH analysis show that two of the echinoderms studied here, Patiriella sp. and S. mollis, do indeed contain SCB (Chapter 3). In both species, SCB occur sporadically throughout the sub-cuticular space, rather than having a uniform distribution. That being said, where SCB are found, they appear to be abundant. No attempt was made to accurately gauge the SCB population size, but often SCB were so densely populated that counting individual bacteria was impossible. No SCB were ever seen in FISH images of A. scabra.

The phylogenetic analysis of bacteria associated with these three echinoderms agrees with the finding of SCB in Patiriella sp. and S. mollis but not A. scabra (Chapter 2). By sequencing a region of the 16s rRNA gene of bacteria from echinoderm tissue and surface samples, as well as seawater-borne bacteria, four species of bacteria were identified as likely candidates for SCB. All four bacteria came from Patiriella sp. or $S$. mollis, and were never found in tissue samples of $A$. scabra. The first putative SCB identified is an $\alpha$-proteobacterium, belonging to the order Rhizobiales, and was isolated from $S$. mollis. The second putative SCB, a $\gamma$-proteobacterium of the order Chromatiales, was isolated from Patiriella sp. and S. mollis. Putative SCB Species 3 is an $\alpha$ proteobacterium belonging to the Roseobacter clade, within the order Rhodobacterales, and was found in S. mollis. The final species identified here as a potential SCB was found in Patiriella sp. and S. mollis, and its phylogenetic grouping is more ambiguous, falling among $\alpha$ - and $\gamma$-proteobacteria. This fourth species is most closely related to members of the orders Rickettsiales and Thiotrichales.

Through the combined use of a eubacterial FISH probe with $\alpha$ - and $\gamma$-proteobacteriaspecific probes, it was determined that the vast majority of SCB in Patiriella sp. and $S$. 
mollis belong to the proteobacteria. More specifically, the SCB community of S. mollis is composed of $\sim 80 \% \alpha$-proteobacteria and $\sim 20 \% \quad \gamma$-proteobacteria. In Patiriella sp. a similar proportion to that seen in $S$. mollis is made up by $\alpha$-proteobacteria $(\sim 80 \%)$, but $\gamma$ proteobacteria were never observed. These results provide some support for the inclusion of the four bacteria mentioned above as putative SCB. Clearly, $\alpha$-proteobacteria make up the majority of the SCB communities of both species of echinoderm, and at least two (possibly three) of the bacteria identified as putative SCB fall within the $\alpha$-proteobacteria. The presence of $\gamma$-proteobacteria in $S$. mollis lends support to the hypothesis that putative SCB species 2 is symbiotic with this echinoderm, as this bacterium falls within the $\gamma$ proteobacteria. The absence of $\gamma$-proteobacteria in FISH images from Patiriella sp. was unexpected, given that putative SCB species 2 was found in both S. mollis and Patiriella sp. However, it seems probable that SCB species 2 is simply rarer than the $\alpha$ proteobacterial SCB and was not found in Patiriella sp. due to an inadequate number of samples being used for FISH. In order to provide conclusive evidence as to the identity of the SCB, FISH probes specific for putative SCB species 1 and 2 were designed, however FISH with these probes was unsuccessful.

Likewise, cultivation of SCB provided inconclusive results, despite utilization of a range of different culture media (Chapter 4). Cultivation of SCB is likely to prove difficult, although the use of selective culture media may be of help; several media suitable for future use are suggested in Chapter 4. 
An obvious goal in studies of symbioses is to determine the function of the relationship; in this case, to understand what, if anything, the bacteria and echinoderms get out of living together. In the next section, potential functions of the four bacteria identified as SCB are considered, based on their phylogenetic affiliations and evidence from previous studies of the echinoderm-SCB symbiosis.

\subsection{Potential roles of $\mathrm{SCB}$ in the symbiosis}

Given the diversity of characters exhibited by bacteria related to the four putative SCB species identified here, the function of the SCB is difficult to determine with certainty. Likewise, it is generally not possible to determine a functional role for bacteria based on sequence information alone. This being said, the relationships of the four putative symbionts identified here to described bacterial species, taken together with previously hypothesized functions of SCB, may shed some light on the role that these bacteria play in the echinoderm-SCB symbiosis. Presently, there are two main hypotheses regarding the function of SCB: that they provide the echinoderm host with a nutritional or energetic benefit (Holland \& Nealson 1978, Lesser \& Walker 1992) (Walker \& Lesser 1989, Roberts et al. 1991, McKenzie \& Kelly 1994, McKenzie et al. 1998), or that they aid in prevention of microbial infection of the host (Dobson 2001, Strahl et al. 2002). There are several mechanisms proposed for the provision of this nutritional benefit. The SCB may take up and use dissolved organic matter (DOM) from the surrounding seawater and transfer metabolic by-products to the host (Walker \& Lesser 1989, Lesser \& Walker 1992), they may be phagocytosed by host cells (Walker \& Lesser 1989, Roberts et al. 1991, McKenzie \& Kelly 1994), or they may be involved in uptake of nitrogen and free 
amino acids from seawater, and the production of proteins (Lesser \& Walker 1992, McKenzie et al. 2000). Direct evidence of phagocytosis of SCB exists from electron microscopy studies (Walker \& Lesser 1989, McKenzie \& Kelly 1994), whereas evidence for any other nutritional or energetic benefit to the host is mostly indirect and somewhat limited to date. There are several exceptions however. Lesser and Walker (1992), for example, demonstrated that SCB of the ophiuroid Amphipholis squamata are able to take up dissolved free amino acids (FAA) from seawater and incorporate them into protein. Similarly, in their stable isotope and fatty acid analyses of several ophiuroids, McKenzie et al. (2000) found that SCB likely contribute to their hosts' nitrogen and fatty acid pools.. It is not known whether any potential provision of nutrients or energy supplements the host as a whole or just the local epidermal tissue, which in some parts can be quite distant from the nutrients transported by the coelom (McKenzie \& Kelly 1994).

The hypothesized antimicrobial action of SCB may also occur in one of several ways. The SCB may be producing an antimicrobial compound which prevents survival of pathogenic bacteria which settle on host wound sites (caused by predation or autotomy) or they may competitively exclude opportunistic pathogens by crowding wound sites (Strahl et al. 2002). Extracts from bacteria associated with an ophiuroid do show antimicrobial activity against a range of bacteria in vitro (in some cases complete inhibition of bacterial growth) (Dobson 2001, Strahl et al. 2002), and very rapid colonization of ophiuroid wound sites by SCB has been observed in brittle stars (McKenzie \& Kelly 1994), supporting the competitive exclusion hypothesis. 
Looking at the results of the present work in the context of these hypotheses, it is possible to suggest potential roles of the four putative SCB identified. Species 1, closely related to numerous nitrogen-fixing bacteria, is likely involved in nitrogen fixation itself. Whether this fixed nitrogen would be transferred to the host is disputable; however comparison with other marine invertebrates lends support to this theory. The gastrointestinal microflora of an echinoid, for example, has been shown to transfer fixed nitrogen to the host (Guerinot \& Patriquin 1981), and similar results have been observed in other marine invertebrates including sponges (Weisz et al. 2007), corals (Kneip et al. 2007) and molluscs (Carpenter \& Culliney 1975). It is likely, in fact, that microbial nitrogen fixation is an important source of nitrogen for many marine invertebrates, as their diets are often composed of low protein food sources such as algae which limit the amount of nitrogen that they are able to obtain from food alone (Guerinot \& Patriquin 1981). Furthermore, the location of SCB within echinoderms may afford the host a better opportunity to utilize the nitrogen fixed by the bacteria. It has been noted that gut contents can be turned over as often as every few hours by actively feeding echinoids, meaning that associated microflora may also be removed quite frequently (Lawrence 1975). While it is possible that gut-associated bacteria are attached to the host and are therefore resistant to removal during gut content turnover (Guerinot \& Patriquin 1981), the relative stability of the sub-cuticular space means that SCB are not in constant danger of removal and may in fact provide a more consistent source of fixed nitrogen. While there is evidence for uptake of nitrogen by SCB in ophiuroids (McKenzie et al. 2000), no SCB have yet been shown to fix nitrogen. However, the presence of nitrogen-fixing 
bacteria in echinoderm guts, and in other marine invertebrates, suggests that it is a possibility.

Species 2 is a member of the order Chromatiales (purple sulfur bacteria), an order which is made up of sulphur-oxidizing autotrophs, as well as several nitrogen-fixing bacteria (Pfennig \& Trüper 1989). It is possible that this species is involved in nitrogen fixation in a similar manner to Species 1. Alternatively, given the autotrophic nature of most Chromatiales bacteria, this species may be providing the host with a different type of nutritional benefit, namely the translocation of chemosynthate in a similar fashion to the translocation of photosynthate from algal symbionts to cnidarian hosts in the coraldinoflagellate symbiosis. A variation on this mechanism, whereby the autotrophic bacteria are simply phagocytosed by host cells is also a possibility here. Phagocytosis of SCB has been observed in other echinoderm species (Walker \& Lesser 1989)(McKenzie \& Kelly 1994), although it is unclear at this stage whether the bacteria were nonselectively phagocytosed, or whether only moribund bacteria were being ingested by the host. Finally, given that bacteria in this order are sulphur-oxidizing, and that hydrogen sulphide is toxic to the aerobic metabolism of animals, species 2 may provide the host with a mechanism for detoxification of such compounds. This mechanism has been suggested for bacterial symbionts of a sediment-feeding echinoderm (Temara et al. 1993), a bivalve mollusc (Vetter 1985) and an oligochaete (phylum Annelida) (Giere \& Langheld 1987), and is equally likely to be occurring in the host species studied here, especially S. mollis, which feeds in the sediment and may be exposed to elevated sulphur levels. Interestingly, in the case of the oligochaete example just given, the bacteria were 
found in the sub-cuticular space of the host (Giere \& Langheld 1987). It is also conceivable that a combination of these mechanisms is at work. For example, the bacteria may be providing the echinoderms with chemosynthetically-fixed $\mathrm{CO}_{2}$ as has been suggested in symbioses between bacteria and bivalves (phylum Mollusca) (Rau \& Hedges 1979) and vestimentiferans (phylum Annelida) (Felbeck 1981), while at the same time oxidizing sulfide, which inhibits cytochrome oxidase, and therefore mitochondrial respiration, in animals (Dorman et al. 2002).

Attempting to assign potential roles to Species 3 and 4 is somewhat more difficult. As mentioned previously, Species 3 falls within the Roseobacter clade, a group with an incredibly diverse range of characteristics. This clade has been subdivided into 41 lineages, and 13 major clusters containing bacteria with different habitats (Buchan et al. 2005). Marine invertebrate-associated bacteria are scattered throughout these clusters, although the only member of this clade so far found in association with an echinoderm comes from a bacterium of the genus Sulfitobacter (Ivanova et al. 2004), which belongs to a different cluster than that identified here. Despite the diversity of habitats and metabolisms present among the different members of this clade, there are several physiologies which are common to many species within the group. Buchan et al. (2005), in their review of marine bacteria belonging to the Roseobacter clade, discuss several of these shared physiologies, such as carbon monoxide oxidation and transformation of certain sulphur compounds. With regards to the symbiosis under study here, one physiology in particular stands out: the production of secondary metabolites, especially those with antibiotic properties. Several bacteria belonging to the genus Roseobacter 
(which is one of the most closely-related genera to Species 3) have been shown to have anti-bacterial action against a range of pathogenic marine bacteria (Brinkhoff et al. 2004, Hjelm et al. 2004). If the hypothesized antibacterial function of SCB is at work in $S$. mollis, perhaps it is achieved through the action of Species 3. An alternative hypothesis is that Species 3 is in fact pathogenic rather than symbiotic. A recent study of skin ulceration disease in the sea cucumber Holothuria scabra identified what appears to be a Roseobacter species from 16S sequences obtained from diseased animals (Becker et al. 2004). Although there was no visible evidence that the sea cucumbers examined in the present study were diseased, it is conceivable that Species 3 is in fact a pathogen present in the cuticle or epidermis of the host. The fact that the animals did not appear diseased could be because of low abundance of this bacterium, that this bacterium causes different symptoms to the one identified in $H$. scabra, or that the putative pathogen is harmless until induced by an external trigger such as stress to the host. It must be clearly stated that both the antimicrobial and pathogenic hypotheses are highly speculative, given the diversity of characters and physiologies possessed by members of the Roseobacter clade. Cultivation and subsequent study of this bacterium are certainly needed if we are to draw any firm conclusion as to what role it may be playing in its putative symbiosis with $S$. mollis.

Species 4 is the most difficult to which to assign a potential symbiotic function. As mentioned previously, this bacterium is most closely related to pathogenic and parasitic bacteria of the genera Piscirickettsia, Francisella and Wolbachia, and paramecium symbionts of the genus Caedibacter. As with Species 3, there is a good chance that 
Species 4 is actually pathogenic rather than symbiotic. While there are reports of echinoderm disease in the literature (Jangoux 1984, Greenstein 1989), there is a paucity of information regarding the causative agents, so it is difficult to draw any conclusions one way or the other. However, the close relationship to so many other pathogenic and parasitic bacteria does lend support to the idea of a pathogenic or parasitic relationship between Species 4 and its hosts, Patiriella sp. and S. mollis. If Species 4 is mutualistic or commensalistic with its hosts, the role it may be playing can only be guessed. A search of the literature reveals no evidence of bacteria in the order Thiotrichales forming such symbioses, and evidence of mutualisms or commensalisms involving bacteria from the order Rickettsiales is restricted to terrestrial hosts such as insects. Rickettsia-like bacteria have been observed in crustaceans (Messick 1998) and molluscs (Herry et al. 1994, Caceres-Martinez \& Tinoco-Orta 2001), however these bacteria seem more likely to be parasitic than mutualistic. Herry et al. (1994), for example, note that the rickettsia-like bacteria seen in the bivalve mollusc Loripes lucinalus may be energy parasites. Based on the limited evidence available, it seems that if Species 4 is not merely a transient pathogen, there is a good chance that the relationship with its echinoderm hosts is parasitic in nature.

The fact that SCB were found in the grazer Patiriella sp. and the deposit-feeding $S$. mollis, but not in the predatory A. scabra lends support to the general notion that SCB provide their hosts with energy or limiting nutrients, whether this be through nitrogen fixation, translocation of DOM or photo- / chemosynthate, or otherwise. A. scabra is a feeds mainly of crustaceans and molluscs (Town 1980), i.e. food sources which are high in energy and nutrients such as nitrogen. Patiriella sp. and S. mollis, on the other hand, 
both subsist on relatively low-energy and low-nitrogen diets: Patiriella sp. predominantly grazes on algae and small barnacles, only occasionally preying upon molluscs (Miller \& Batt 1973), and S. mollis obtains food from ingested sediment and organic detritus (Brusca \& Brusca 2003). It is clearly evident from looking at the feeding habits of these three species that if SCB were to provide their host with a nutritional or energetic benefit, they would more likely be found in species such as Patiriella sp. and S. mollis, as is found to be the case here. Further evidence comes from McKenzie et al. (1998), who note that echinoderm families such as Asteriidae, which are highly carnivorous, lack SCB, while those families that do tend to contain SCB have a more diverse feeding habit. That being said, in the two largest studies of the distribution of SCB to date, no relationship between SCB presence and host ecology was found (Kelly et al. 1995, Kelly \& McKenzie 1995). It would therefore be imprudent to suggest a link between SCB presence and feeding habit based on the few species studied here, but it is an interesting observation nonetheless.

The results of the present study do not provide any information regarding the benefits to the SCB from the symbiosis. It has been suggested that the bacteria may obtain DOM that is leaked from host cells (Holland \& Nealson 1978, Feral 1980), and they would certainly be in a good position to do so, given their close proximity to the epidermal cells of the host. Additionally, their location beneath the cuticle of their hosts presumably makes SCB are less vulnerable to grazing. Further work is needed before drawing any conclusions about the benefit gained by the bacteria in this symbiosis. 


\subsection{Future directions}

While this study has resulted in much new information about the phylogenetic affiliations of the putative SCB found in Patiriella sp. and S. mollis, it has only scratched the surface of what there is to be learned about this symbiosis. Due to the general lack of information about the echinoderm-SCB symbiosis, there are many avenues of research which remain to be explored. Here, four general areas of future research, which represent the most logical next steps, are discussed.

The immediate way forward is to utilize the methods developed in the present study to confirm the accuracy of the results obtained here. Four species of bacteria were identified as potential $\mathrm{SCB}$, but it must be stressed that this is by no means a definitive result. As stated in Chapter 2, the presence of vast numbers of potentially contaminating bacteria in environmental studies such as this means that the four bacteria identified cannot be confirmed as SCB. Likewise, the possibility exists that there are other bacteria, not identified here, which are in fact SCB. The simplest way to accurately determine the identity of SCB is through the use of bacterial species-specific FISH probes. Due to time constraints, only two specific probes were used in the present study, and neither successfully hybridized, possibly due to inaccessibility of the probe target site. However, given more time, a range of specific probes could be designed, and if used in conjunction with a eubacterial probe, the proportion of the SCB community made up by each species of bacterium could be accurately determined. In cases where specific FISH probes show the presence of unknown bacteria (i.e. where bacteria are labeled by the eubacterial probe but not the specific probes), bacterial DNA sequences from further echinoderm samples 
may be required. With enough time, this combined use of sequencing / phylogenetic analysis and FISH should lead to accurate identification of SCB in Patiriella sp. and S. mollis.

Once the SCB of Patiriella sp. and S. mollis have been identified with some certainty, the study can be expanded to other echinoderm species, in order to see whether or not the same species of SCB are present in different echinoderms. The expansion of the study to other populations of Patiriella sp. and S. mollis, and sampling during different seasons would also be beneficial, so that any variation in SCB community structure across time or space can be assessed.

The third recommended area of research is tied in with the previous one. By expanding the study to a sufficiently large and diverse range of echinoderms, it should be possible to map the phylogeny of echinoderms alongside the phylogeny of their SCB. By doing this, some light may be shed on the mode of transmission of SCB. If echinoderm-specific clusters of bacteria are found which are tightly linked with host phylogeny, it would provide support for the hypothesis of vertical (i.e. parent to offspring) transmission. Transfection experiments, such as those carried out by Barker and Kelly (1994), would also be helpful in establishing whether or not SCB are transmitted from parent to offspring, as would the use of FISH to test for the presence of SCB in the gametes or larval stages of host species. 
The final, and perhaps most interesting, recommended research direction is attempting to elucidate the function of SCB. While some suggestions were made in the preceding section as to the possible roles of the putative SCB identified here, these are highly speculative, being based on 16S rDNA sequence data only. Traditionally, the first step in determining the function of bacteria such as SCB would involve cultivation. As has been shown here, this is not a straightforward task. While it is likely that cultivation of SCB is possible, it will most probably take considerable time and effort. Therefore, the use of metagenomics may offer a promising alternative. Metagenomics (also known as environmental genomics) allows us to gain nearly complete genomes from bacteria present in an environmental sample. With such data at hand, much information can be obtained about those bacteria which are identified as SCB. For example, the presence of high numbers of a particular gene in a SCB genome suggests that whatever the gene codes for is an important part the bacterium's physiology. For instance, if a gene responsible for the production of antimicrobial metabolites was present in high numbers, it may provide support for the hypothesis that SCB produce an antimicrobial compound which is beneficial to the echinoderm host. Similarly, if a SCB is found to have undergone gene loss (i.e. it has far fewer genes than its close non-symbiotic relatives), this would be evidence for vertical transmission of the SCB from host parent to offspring, and co-evolution of SCB and host, as gene loss generally indicates that a bacterium is obligately symbiotic. Finally, the use of mRNA expression profiling will allow us to better understand the physiology of SCB by determining which genes are expressed more than others. For further information about the metagenomic techniques above, see the excellent review of environmental genomics by Bertin et al (2008). 
Through the use of the full range of methods described above, combined with the knowledge gained from this study and others, it may be possible to finally determine the function, diversity and mode of symbiont transmission in the echinoderm-SCB symbiosis. Given the scarcity of research into this relationship over the last 30 years, and the recent advent of metagenomics and other molecular techniques, now is the time to properly investigate this potentially important symbiosis. 


\section{References}

Allen EE, Banfield JF (2005) Community genomics in microbial ecology and evolution. Nature Reviews Microbiology 3:489-498

Althoff K, Schütt C, Steffen R, Batel R, Müller WEG (1998) Evidence for a symbiosis between bacteria of the genus Rhodobacter and the marine sponge Halichondria panicea: harbor also for putatively toxic bacteria? Marine Biology 130:529-536

Altschul SF, Gish W, Miller W, Myers EW, Lipman DJ (1990) Basic local alignment search tool. Journal of Molecular Biology 215:403-410

Amann RI, Binder BJ, Olson RJ, Chisholm SW, Devereux R, Stahl DA (1990) Combination of 16S rRNA-targeted oligonucleotide probes with flow cytometry for analyzing mixed microbial populations. Applied and Environmental Microbiology 56:1919-1925

Amaro AM, Fuentes MS, Ogalde SR, Venegas JA, Suárez-Isla BA (2005) Identification and characterization of potentially algal-lytic marine bacteria strongly associated with the toxic dinoflagellate Alexandrium catenella. The Journal of Eukaryotic Microbiology 52:191-200

Anderson CM, Haygood MG (2007) $\alpha$-proteobacterial symbionts of marine bryozoans in the genus Watersipora. Applied and Environmental Microbiology 73:303-311

Arndt C, Schiedek D (1997) Nephtys hombergii, a free-living predator in marine sediments: energy production under environmental stress. Marine Biology $129: 643-650$ 
Ashton M, Rosado W, Govind NS, Tosteson TR (2003) Culturable and nonculturable bacterial symbionts in the toxic benthic dinoflagellate Ostreopsis lenticularis. Toxicon 42:419-424

Atlas RM (2005) Handbook of media for environmental microbiology, Vol. CRC Press, Boca Raton, FL

Baker GC, Smith JJ, Cowan DA (2003) Review and re-analysis of domain-specific 16S primers. Journal of Microbiological Methods 55:541-555

Bandi C, McCall JW, Genchi C, Corona S, Venco L, Sacchi L (1999) Effects of tetracycline on the filarial worms Brugia pahangi and Dirofilaria immitis and their bacterial endosymbionts Wolbachia. International Journal for Parasitology 29:357-364

Barbieri EP, Bruce J, Hughes D, Zurek L, Moser DP, Teske A, Sogin ML (2001) Phylogenetic characterization of epibiotic bacteria in the accessory nidamental gland and egg capsules of the squid Loligo pealei (Cephalopoda: Loliginidae). Environmental Microbiology 3:151-167

Barker MF, Kelly M (1994) The occurrence and transmission of subcuticular bacteria in echinoderm larvae. In: David B, Guille A, Feral JP, Roux M (eds) Echinoderms through Time. Balkema, Rotterdam, p 13

Baumann P, Gauthier MJ, Baumann L (1984) Genus Alteromonas Baumann, Baumann, Mandel and Allen 1972, 418. In: Krieg NR (ed) Bergey's Manual of Systematic Bacteriology, Vol 1. Williams \& Wilkins, Baltimore, MD, p 343-352 
Bavington CD, Lever R, Mulloy B, Grundy MM, Page CP, Richardson NV, McKenzie JD (2004) Anti-adhesive glycoproteins in echinoderm mucus secretions. Comparative Biochemistry and Physiology, Part B 139:607-617

Becker P, Gillan D, Lanterbecq D, Jangoux M, Rasolofonirina R, Rakotovao J, Eeckhaut I (2004) The skin ulceration disease in cultivated juveniles of Holothuria scabra (Holothuroidea, Echinodermata). Aquaculture 242:13-30

Beier CL, Horn M, Michel R, Schweikert M, Görtz H-D, Wagner M (2002) The genus Caedibacter comprises endosymbionts of Paramecium spp. related to the Rickettsiales (Alphaproteobacteria) and to Francisella tularensis (Gammaproteobacteria). Applied and Environmental Microbiology 68:6043-6050

Bell PJL (2001) Viral eukaryogenesis: was the ancestor of the nucleus a complex DNA virus? Journal of Molecular Evolution 53:251-256

Bertin PN, Médigue C, Normand P (2008) Advances in environmental genomics: towards an integrated view of micro-organisms and ecosystems. Microbiology $154: 347-359$

Blazejak A, Erséus C, Amann R, Dubilier N (2005) Coexistence of bacterial sulfide oxidizers, sulfate reducers, and spirochetes in a gutless worm (Oligochaeta) from the Peru margin. Applied and Environmental Microbiology 71:1553-1561

Blunt JW, Copp BR, Munro MH, Northcote PT, Prinsep MR (2005) Marine natural products. Natural Product Reports 22:15-61

Blunt JW, Copp BR, Munro MH, Northcote PT, Prinsep MR (2006) Marine natural products. Natural Product Reports 23:26-78 
Boettcher KJ, Barber BJ, Singer JT (2000) Additional evidence that juvenile oyster disease is caused by a member of the Roseobacter group and colonization of nonaffected animals by Stappia stellulata-like strains. Applied and Environmental Microbiology 66:3924-3930

Bosch I (1992) Symbiosis between bacteria and oceanic clonal sea star larvae in the western North Atlantic Ocean. Marine Biology 114:495-502

Bourne DG, Munn CB (2005) Diversity of bacteria associated with the coral Pocillopora damicornis from the Great Barrier Reef. Environmental Microbiology 7:11621174

Bowman JP (2006) The Genus Psychrobacter. In: Dworkin M, Falkow S, Rosenberg E, Schleifer KH, Stackebrandt E (eds) The Prokaryotes, Vol 6. Springer-Verlag, New York, NY, p 920-930

Bozal N, Montes MJ, Tudela E, Guinea J (2003) Characterization of several Psychrobacter strains isolated from Antarctic environments and description of Psychrobacter luti sp. nov. and Psychrobacter fozii sp. nov. International Journal of Systematic and Evolutionary Microbiology 53:1093-1100

Brantley SE, Molinski TF, Preston CM, DeLong EF (1995) Brominated acetylenic fatty acids from Xestopongia sp., a marine sponge-bacteria association. Tetrahedron $51: 7667-7672$

Brigmon RL, de Ridder C (1998) Symbiotic relationship of Thiothrix spp. with an echinoderm. Applied and Environmental Microbiology 64:3491-3495

Brinkhoff T, Bach G, Heidorn T, Liang F, Schlingloff A, Simon M (2004) Antibiotic production by a Roseobacter clade-affiliated species from the German Wadden 
Sea and its antagonistic effects on indigenous isolates. Applied and Environmental Microbiology 70:2560-2565

Brinkhoff T, Giebel H-A, Simon M (2008) Diversity, ecology, and genomics of the Roseobacter clade: a short overview. Archives of Microbiology 189:531-539

Brinkmeyer R, Rappé M, Gallacher S, Medlin L (2000) Development of clade(Roseobacter and Alteromonas) and taxon-specific oligonucleotide probes to study interactions between toxic dinoflagellates and their associated bacteria. European Journal of Phycology 35:315-329

Brusca RC, Brusca GJ (2003) Invertebrates, Vol. Sinauer Associates, Sunderland, MA

Bryan PJ, Rittschof D, McClintock JB (1996) Bioactivity of echinoderm ethanolic bodywall extracts: an assessment of marine bacterial attachment and macroinvertebrate larval settlement. Journal of Experimental Marine Biology and Ecology 196:7996

Buchan A, Gonzalez JM, Moran MA (2005) Overview of the marine Roseobacter lineage. Applied and Environmental Microbiology 71:5665-5677

Burnett WJ, McKenzie JD (1997) Subcuticular bacteria from the brittle star Ophiactis balli (Echinodermata: Ophiuroidea) represent a new lineage of extracellular marine symbionts in the $\alpha$ subdivision of the class Proteobacteria. Applied and Environmental Microbiology 63:1721-1724

Caceres-Martinez J, Tinoco-Orta GD (2001) Symbionts of cultured red abalone Haliotis rufescens from Baja California, Mexico. Journal of Shellfish Research 20:875881 
Carpenter EJ, Culliney JL (1975) Nitrogen fixation in marine shipworms. Science $187: 551-552$

Cary SC, Warren W, Anderson E, Giovannoni SJ (1993) Identification and localization of bacterial endosymbionts in hydrothermal vent taxa with symbiont-specific polymerase chain reaction amplification and in situ hybridization techniques. Molecular Marine Biology and Biotechnology 2:51-82

Cavanaugh CM, Gardiner SL, Jones ML, Jannasch HW, Waterbury JB (1981) Prokaryotic cells in the hydrothermal vent tube worm Riftia pachyptila Jones: Possible chemoautotrophic symbionts. Science 213:340-342

Chao LSL, Davis RE, Moyer CL (2007) Characterization of bacterial community structure in vestimentiferan tubeworm Ridgeia piscesae trophosomes. Marine Ecology 28:72-85

Cooney RP, Pantos O, Le Tissier MD, Barer MR, O'Donnell AG, Bythell JC (2002) Characterization of the bacterial consortium associated with black band disease in coral using molecular microbiological techniques. Environmental Microbiology $4: 401-413$

Costello MJ, Bouchet P, Emblow CS, Legakis A (2006) European marine biodiversity inventory and taxonomic resources: state of the art and gaps in knowledge. Marine Ecology Progress Series 316:257-268

Da Silva SG, Gillan DC, Dubilier N, De Ridder C (2006) Characterization by 16s rRNA gene analysis and in situ hybridization of bacteria living in the hindgut of a deposit-feeding echinoid (Echinodermata). Journal of the Marine Biological Association of the United Kingdom 86:1209-1213 
Dafny-Yelin M, Levy A, Tzfira T (2008) The ongoing saga of Agrobacterium-host interactions. Trends in Plant Science 13:102-105

Davidson SK, Allen SW, Lim GE, Anderson CM, Haygood MG (2001) Evidence for the biosynthesis of bryostatins by the bacterial symbiont "Candidatus Endobugula sertula" of the bryozoan Bugula neritina. Applied and Environmental Microbiology 67:4531-4537

Davy JE, Patten NL (2007) Morphological diversity of virus-like particles within the surface microlayer of scleractinian corals. Aquatic Microbial Ecology 47:37-44

De Ridder C, Jangoux M (1985) Description and significance of a peculiar intradigestive symbiosis between bacteria and a deposit-feeding echinoid. Journal of Experimental Marine Biology and Ecology 91:65-76

Dedeine F, Vavre F, Fleury F, Loppin B, Hochberg ME, Bouletreau M (2001) Removing symbiotic Wolbachia bacteria specifically inhibits oogenesis in a parasitic wasp. Proceedings of the National Academy of Sciences 98:6247-6252

Deming JW, Colwell RR (1982) Barophilic bacteria associated with digestive tracts of abyssal holothurians. Applied and Environmental Microbiology 44:1222-1230

Desbruyères D, Chevaldonné P, Alayse AM, Jollivet D, Lallier FH, Jouin-Toulmond C, Zal F, Sarradin PM, Cosson R, Caprais JC, Arndt C, O'Brien J, Guezennec J, Hourdez S, Riso R, Gaill F, Laubier L, Toulmond A (1998) Biology and ecology of the "Pompeii worm" (Alvinella pompejana Desbruyères and Laubier), a normal dweller of an extreme deep-sea environment: A synthesis of current knowledge and recent developments. Deep-Sea Research I 45:383-422 
Dimitrieva GY, Dimitriev SM (1996) Symbiotic microflora of brown algae of the genus Laminaria as a bioindicator of the ecological condition of coastal laminarian biocenoses. Russian Journal of Marine Biology 22:276-281

Distel DL, Felbeck H, Cavanaugh CM (1994) Evidence for phylogenetic congruence among sulfur-oxidizing chemoautotrophic bacterial endosymbionts and their bivalve hosts. Journal of Molecular Evolution 38:533-542

Distel DL, Lane DJ, Olsen GJ, Giovannoni SJ, Pace B, Pace NR, Stahl DA, Felbeck H (1988) Sulfur-oxidizing bacterial endosymbionts: analysis of phylogeny and specificity by $16 \mathrm{~S}$ rRNA sequences. Journal of Bacteriology 170:2506-2510

Distel DL, Lee HKW, Cavanaugh CM (1995) Intracellular coexistence of methano- and thioautotrophic bacteria in a hydrothermal vent mussel. Proceedings of the National Academy of Sciences 92:9598-9602

Dobretsov S, Qian PY (2004) The role of epibiotic bacteria from the surface of the soft coral Dendronephthya sp. in the inhibition of larval settlement. Journal of Experimental Marine Biology and Ecology 299:35-50

Dobson WE (2001) Isolation and screening of subcuticular brittlestar bacteria for antimicrobial compounds production. In: Office USPaT (ed). Appalachian State University, USA

Dorman DC, Moulin FJ-M, McManus BE, Mahle KC, Arden James R, Struve MF (2002) Cytochrome oxidase inhibition induced by acute hydrogen sulfide inhalation: correlation with tissue sulfide concentrations in the rat brain, liver, lung, and nasal epithelium. Toxicological Sciences 65:18-25 
Douglas AE (2003) Coral bleaching - how and why? Marine Pollution Bulletin 46:385392

Duperron S, Sibuet M, MacGregor BJ, Kuypers MMM, Fisher CR, Dubilier N (2007) Diversity, relative abundance and metabolic potential of bacterial endosymbionts in three Bathymodiolus mussel species from cold seeps in the Gulf of Mexico. Environmental Microbiology 9:1423-1438

Durand P, Gros O, Frenkiel L, Prieur D (1996) Phylogenetic characterization of sulfuroxidizing bacterial endosymbionts in three tropical Lucinidae by $16 \mathrm{~s}$ rDNA sequence analysis. Molecular Marine Biology and Biotechnology 5:37-42

Elsaied HE, Kaneko R, Naganuma T (2006) Molecular characterization of a deep-sea methanotrophic mussel symbiont that carries a RuBisCO gene. Marine Biotechnology 8:511-520

Felbeck H (1981) Chemoautotrophic potentials of the hydrothermal vent tube worm Riftia pachyptila (Vestimentifera). Science 213:336-338

Feral J-P (1985) Effect of short-term starvation on the biochemical composition of the apodous holothurian Leptosynapta galliennei (Echinodermata): possible role of dissolved organic material as an energy source. Marine Biology 86:297-306

Feral JP (1980) Cuticule et bactéries associées des Epidermes digestif et tégumentaire de Leptosynapta galliennei (Herapath) (Holothuroidea: Apoda) - Premières données. In: Jangoux M (ed) Echinoderms: present and past. Balkema, Rotterdam, p 285290 
Fieseler L, Quaiser A, Schleper C, Hentschel U (2004) Discovery of the novel candidate phylum "Poribacteria" in marine sponges. Applied and Environmental Microbiology 70:3724-3732

Foret TW, Lawrence JM (2001) Variation in abundance of subcuticular bacteria in Florida echinoderms. Symbiosis 31:309-322

Frenkiel L, Gros O, Mouëza M (1996) Gill ultrastructure in Lucina pectinata (Bivalvia: Lucinidae) with reference to hemoglobin in bivalves with symbiotic sulphuroxidizing bacteria. Marine Biology 125:511-524

Frischer ME, Floriani PJ, Nierzwicki-Bauer SA (1996) Differential sensitivity of 16s rRNA targeted oligonucleotide probes used for fluorescence in situ hybridization is a result of ribosomal higher order structure. Canadian Journal of Microbiology 42:1061-1071

Fry JC (2004) Culture-dependent microbiology. In: Bull AT (ed) Microbial Diversity and Bioprospecting. ASM Press, Washington, D.C., p 80-87

Fryer JL, Lannan CN, Giovannoni SJ, Wood ND (1992) Piscirickettsia salmonis gen. nov., sp. nov., the causative agent of an epizootic disease in salmonid fishes. International Journal of Systematic Bacteriology 42:120-126

Fuchs BM, Glöckner FO, Wulf J, Amann R (2000) Unlabeled helper oligonucleotides increase the in situ accessibility to $16 \mathrm{~s}$ rRNA of fluorescently labeled oligonucleotide probes. Applied and Environmental Microbiology 66:3603-3607

Fuchs BM, Pernthaler J, Amann R (2007) Single cell identification by fluorescence in situ hybridization. In: Reddy CA (ed) Methods for general and molecular microbiology. ASM Press, Washington, D.C., p 886-896 
Gagnon P, Himmelman JH, Johnson LE (2003) Algal colonization in urchin barrens: defense by association during recruitment of the brown alga Agarum cribrosum. Journal of Experimental Marine Biology and Ecology 290:179-196

Giere O, Langheld C (1987) Structural organisation, transfer and biological fate of endosymbiotic bacteria in gutless oligochaetes. Marine Biology 93:641-650

Gil-Turnes MS, Hay ME, Fenical W (1989) Symbiotic marine bacteria chemically defend crustacean embryos from a pathogenic fungus. Science 246:116-118

Gini GA (1990) Ocular infection caused by Psychrobacter immobilis acquired in the hospital. Journal of Clinical Microbiology 28:400-401

Goffredi SK, Orphan VJ, Rouse GW, Jahnke L, Embaye T, Turk K, Lee R, Vrijenhoek RC (2005) Evolutionary innovation: a bone-eating marine symbiosis. Environmental Microbiology 7:1369-1378

Gonzalez JM, Simo R, Massana R, Covert JS, Casamayor EO, Pedros-Alio C, Moran MA (2000) Bacterial community structure associated with a dimethylsulfoniopropionate-producing North Atlantic algal bloom. Applied and Environmental Microbiology 66:4237-4246

Gray MW (1989) The evolutionary origins of organelles. Trends in Genetics 5:294-299

Greenstein BJ (1989) Mass mortality of the West-Indian echinoid Diadema antillarum (Echinodermata: Echinoidea): a natural experiment in taphonomy. Palaios 4:487492

Grimmer JC, Holland ND (1990) The structure of a sessile, stalkless crinoid (Holopus rangii). Acta zoologica 71:61-67 
Groben R, Doucette GJ, Kopp M, Kodama M, Amann R, Medlin LK (2000) 16s rRNA targeted probes for the identification of bacterial strains isolated from cultures of the toxic dinoflagellate Alexandrium tamarense. Microbial Ecology 39:186-196

Guenther J, Walker-Smith G, Waren A, de Nys R (2007) Fouling-resistant surfaces of tropical sea stars. Biofouling 23:413-418

Guerinot ML, Patriquin DG (1981) The association of N2-fixing bacteria with sea urchins. Marine Biology 62:197-207

Guttigoli A, Zaman MM (2000) Bacteremia and possible endocarditis caused by Moraxella phenylpyruvica. Southern Medical Journal 93:708-709

Haine ER (2008) Symbiont-mediated protection. Proceedings of the Royal Society B: Biological Sciences 275:353-361

Hale KJ, Hummersone MG, Manaviazar S, Frigerio M (2002) The chemistry and biology of the bryostatin antitumour macrolides. Natural Product Reports 19:413-453

Hau HH, Gralnick JA (2007) Ecology and biotechnology of the genus Shewanella. Annual Review of Microbiology 61:237-258

Haygood MG, Schmidt EW, Davidson SK, Faulkner DJ (1999) Microbial symbionts of marine invertebrates: Opportunities for microbial biotechnology. Journal of Molecular Microbiology and Biotechnology 1:33-43

Hentschel U, Schmid M, Wagner M, Fieseler L, Gernert C, Hacker J (2001) Isolation and phylogenetic analysis of bacteria with antimicrobial activities from the Mediterranean sponges Aplysina aerophoba and Aplysina cavernicola. FEMS Microbiology Ecology 35:305-312 
Hentschel U, Usher KM, Taylor MW (2006) Marine sponges as microbial fermenters. FEMS Microbiology Ecology 55:167-177

Herry A, Le Pennec M, Johnson M (1994) Bacteria-host relationships in the bivalve mollusc Loripes lucinalis. Acta microbiologica et immunologica Hungarica 41:273-281

Hertig M (1936) The rickettsia, Wolbachia pipientis (Gen. et Sp. Nov.) and associated inclusions of the mosquito, Culex pipiens. Parasitology 28:453-486

Hisar O, Yanik T, Hisar SA (2002) Clinical and pathological investigation of Psychrobacter immobilis infection in rainbow trout (Oncorhynchus mykiss, Walbaum). Israeli Journal of Aquaculture - Bamidgeh 54:189-196

Hjelm M, Bergh O, Riaza A, Nielsen J, Melchiorsen J, Jensen S, Duncan H, Ahrens P, Birkbeck TH, Gram L (2004) Selection and identification of autochthonous potential probiotic bacteria from turbot larvae (Scophthalmus maximus) rearing units. Systematic and Applied Microbiology 27:360-371

Hoegh-Guldberg O (1999) Climate change, coral bleaching and the future of the world's coral reefs. Marine and Freshwater Research 50:839-866

Holland ND, Grimmer JC, Wiegmann K (1991) The structure of the sea lily Calamocrinus diomedae, with special reference to the articulations, skeletal microstructure, symbiotic bacteria, axial organs, and stalk tissues (Crinoidea, Millericrinida). Zoomorphology 110:115-132

Holland ND, Nealson KH (1978) The fine structure of the echinoderm cuticle and the subcuticular bacteria of echinoderms. Acta zoologica 59:169-185 
House ML, Hedrick RP, Winton JR, Fryer JL (2006) An isolate of Piscirickettsia salmonis from white seabass is fully virulent for coho salmon. Journal of Aquatic Animal Health 18:252-256

Huber T, Faulkner G, Hugenholtz P (2004) Bellerophon; a program to detect chimeric sequences in multiple sequence alignments. Bioinformatics 20:2317-2319

Hugenholtz P (2002) Exploring prokaryotic diversity in the genomic era. Genome Biology 3:1-8

Ivanova EP, Gorshkova NM, Sawabe T, Zhukova NV, Hayashi K, Kurilenko VV, Alexeeva Y, Buljan V, Nicolau DV, Mikhailov VV, Christen R (2004) Sulfitobacter delicatus sp. nov. and Sulfitobacter dubius sp. nov., respectively from a starfish (Stellaster equestris) and sea grass (Zostera marina). International Journal of Systematic and Evolutionary Microbiology 54:475-480

Jangoux M (1984) Diseases of echinoderms. Helgoländer Meeresuntersuchungen 37:207216

Jayatilake GS, Thornton MP, Leonard AC, Grimwade JE, Baker BJ (1996) Metabolites from an antarctic sponge-associated bacterium, Pseudomonas aeruginosa. Journal of Natural Products 59:293-296

Jorquera MA, Riquelme CE, Loyola LA, Muñoz LF (1999) Production of bactericidal substances by a marine vibrio isolated from cultures of the scallop Argopecten purpuratus. Aquaculture International 7:433-448

Juretschko S, Buccat AM, Fritsche TR (2004) Applications of fluorescence in situ hybridization in diagnostic microbiology. In: Persing DH, Tenover FC, Versalovic J, Tang Y-W, Unger ER, Relman DA, White TJ (eds) Molecular 
microbiology: diagnostic principles and practice. ASM Press, Washington, D.C., p 3-18

Kan FL, Chen ZY, Wang ET, Tian CF, Sui XH, Chen WX (2007) Characterization of symbiotic and endophytic bacteria isolated from root nodules of herbaceous legumes grown in Qinghai-Tibet plateau and in other zones of China. Archives of Microbiology 188:103-115

Keim P, Johansson A, Wagner DM (2007) Molecular epidemiology, evolution, and ecology of Francisella. Annals of the New York Academy of Sciences 1105:3066

Kelly MS, Barker MF, McKenzie JD, Powell J (1995) The incidence and morphology of subcuticular bacteria in the echinoderm fauna of New Zealand. Biological Bulletin 189:91-105

Kelly MS, McKenzie JD (1992) The quantification of sub-cuticular bacteria in echinoderms. In: Scalera-Liaci L, Canicatti C (eds) Echinoderm Research 1991. Balkema, Rotterdam, Salem, NH, p 225-228

Kelly MS, McKenzie JD (1995) Survey of the occurrence and morphology of subcuticular bacteria in shelf echinoderms from the north-east Atlantic Ocean. Marine Biology 123:741-756

Kelly MS, McKenzie JD, Barker MF (1994) Sub-cuticular bacteria: Their incidence in the echinoderms of the British Isles and New Zealand. In: David B, Guille A, Feral J-P, Roux M (eds) Eighth International Echinoderm Conference. Balkema, Rotterdam, Dijon, France, p 33-38 
Klaschik S, Lehmann LE, Raadts A, Book M, Hoeft A, Stuber F (2002) Real-time PCR for detection and differentiation of Gram-positive and Gram-negative bacteria. Journal of Clinical Microbiology 40:4304-4307

Kneip C, Lockhart P, Voss C, Maier UG (2007) Nitrogen fixation in eukaryotes - New models for symbiosis. BMC Evolutionary Biology 7:55

Kogel KH, Franken P, Hückelhoven R (2006) Endophyte or parasite - what decides? Current Opinion in Plant Biology 9:358-363

König GM, Kehraus S, Seibert SF, Abdel-Lateff A, Müller D (2006) Natural products from marine organisms and their associated microbes. ChemBioChem 7:229-238

Koren O, Rosenberg E (2006) Bacteria associated with mucus and tissues of the coral Oculina patagonica in summer and winter. Applied and Environmental Microbiology 72:5254-5259

Krick A, Kehraus S, Eberl L, Riedel K, Anke H, Kaesler I, Graeber I, Szewzyk U, König GM (2007) A marine Mesorhizobium sp. produces structurally novel long -chain N-acyl-1-homoserine lactones. Applied and Environmental Microbiology $73: 3587-3594$

Krieg NR, Holt JG (eds) (1984) Bergey's manual of systematic bacteriology, Vol 1. Williams \& Wilkins, Baltimore, MD

Krueger DM, Cavanaugh CM (1997) Phylogenetic diversity of bacterial symbionts of Solemya hosts based on comparative sequence analysis of 16s rRNA genes. Applied and Environmental Microbiology 63:91-98 
Krueger DM, Gustafson RG, Cavanaugh CM (1996) Vertical transmission of chemoautotrophic symbionts in the bivalve Solemya velum (Bivalvia:Protobranchia). Biological Bulletin 190:195-202

Kutschera U, Niklas KJ (2005) Endosymbiosis, cell evolution, and speciation. Theory in Biosciences 124:1-24

Lane DJ (1991) 16S/23S rRNA sequencing. In: Stackebrandt E, Goodfellow M (eds) Nucleic acid techniques in bacterial systematics. Wiley, Chichester, U.K., p 115175

Lawrence JM (1975) On the relationships between marine plants and sea urchins. Oceanography and Marine Biology 13:213-286

Lee K, Lee HK, Choi TH, Kim KM, Cho JC (2007) Granulosicoccaceae fam. nov., to include Granulosicoccus antarcticus gen. nov., sp. nov., a non-phototrophic, obligately aerobic chemoheterotroph in the order chromatiales, isolated from antarctic seawater Journal of Microbiology and Biotechnology 17:1483-1490

Lee OO, Qian PY (2004) Potential control of bacterial epibiosis on the surface of the sponge Mycale adhaerens Aquatic Microbial Ecology 34:11-21

Leonardo MR, Moser DP, Barbieri E, Brantner CA, MacGregor BJ, Paster BJ, Stackebrandt E, Nealson KH (1999) Shewanella pealeana sp. nov., a member of the microbial community associated with the accessory nidamental gland of the squid Loligo pealei. International Journal of Systematic Bacteriology 49:13411351 
Lesser MP, Blakemore RP (1990) Description of a novel symbiotic bacterium from the brittle star, Amphipholis squamata. Applied and Environmental Microbiology $56: 2436-2440$

Lesser MP, Mazel CH, Gorbunov MY, Falkowski PG (2004) Discovery of symbiotic nitrogen-fixing cyanobacteria in corals. Science 305:997-1000

Lesser MP, Walker CW (1992) Comparative study of the uptake of dissolved amino acids in sympatric brittle stars with and without endosymbiotic bacteria. Comparative Biochemistry and Physiology, Part B 101:217-223

Lopanik N, Gustafson KR, Lindquist N (2004) Structure of bryostatin 20: A symbiontproduced chemical defense for larvae of the host bryozoan, Bugula neritina. Journal of Natural Products 67:1412-1414

Loy A, Maixner F, Wagner M, Horn M (2007) probeBase - an online resource for rRNAtargeted oligonucleotide probes: new features 2007. Nucleic Acids Research 35:D800-804

Ludwig W, Strunk O, Westram R, Richter L, Meier H, Kumar Y, Buchner A, Lai T, Steppi S, Jobb G, Förster W, Brettske I, Gerber S, Ginhart AW, Gross O, Grumann S, Hermann S, Jost R, König A, Liss T, Lüssmann R, May M, Nonhoff B, Reichel B, Strehlow R, Stamatakis A, Stuckmann N, Vilbig A, Lenke M, Ludwig T, Bode A, Schleifer KH (2004) ARB: a software environment for sequence data. Nucleic Acids Research 32:1363-1371

Maidak BL, Cole JR, Lilburn TG, Parker CT, Saxman PR, Farris RJ, Garrity GM, Olsen GJ, Schmidt TM, Tiedje JM (2001) The RDP-II (Ribosomal Database Project). Nucleic Acids Research 29:173-174 
Maloy AP, Ford SE, Karney RC, Boettcher KJ (2007) Roseovarius crassostreae, the etiological agent of Juvenile Oyster Disease (now to be known as Roseovarius Oyster Disease) in Crassostreae virginica. Aquaculture 269:71-83

Manz W, Amann R, Ludwig W, Wagner M, Schleifer KH (1992) Phylogenetic oligodeoxynucleotide probes for the major subclasses of Proteobacteria: problems and solutions. Systematic and Applied Microbiology 15:593-600

Margulis L (1981) Symbiosis in cell evolution, Vol. W.H. Freeman, San Francisco

McFadden GI (1999) Endosymbiosis and evolution of the plant cell. Current Opinion in Plant Biology 2:513-519

Mcfall-Ngai MJ (1999) Consequences of evolving with bacterial symbionts: insights from the squid-vibrio associations. Annual Review of Ecology and Systematics $30: 235-256$

McKenzie JD, Black KD, Kelly MS, Newton LC, Handley LL, Scrimgeour CM, Raven JA, Henderson RJ (2000) Comparisons of fatty acid and stable isotope ratios in symbiotic and non-symbiotic brittlestars from Oban Bay, Scotland. Journal of the Marine Biological Association of the United Kingdom 80:311-320

McKenzie JD, Burnett WJ, Kelly MS (1998) Systematic distribution of subcuticular bacteria in echinoderms. In: Mooi R, Telford M (eds) Echinoderms: San Francisco. Balkema, Rotterdam, San Francisco, p 53-59

McKenzie JD, Grigolava IV (1996) The echinoderm surface and its role in preventing microfouling. Biofouling 10:261-272

McKenzie JD, Kelly MS (1994) Comparative study of sub-cuticular bacteria in brittlestars (Echinodermata: Ophiuroidea). Marine Biology 120:65-80 
Messick GA (1998) Diseases, parasites, and symbionts of Blue Crabs (Callinectes sapidus) dredged from Chesapeake Bay. Journal of Crustacean Biology 18:533548

Meziti A, Kormas KA, Pancucci-Papadopoulou MA, Thessalou-Legaki M (2007) Bacterial phylotypes associated with the digestive tract of the sea urchin Paracentrotus lividus and the ascidian Microcosmus sp. Russian Journal of Marine Biology 33:84-91

Mikhailov VV, Romanenko LA, Ivanova EP (2006) The Genus Alteromonas and Related Proteobacteria. In: Dworkin M, Falkow S, Rosenberg E, Schleifer KH, Stackebrandt E (eds) The Prokaryotes, Vol 6. Springer-Verlag, New York, NY, p $597-645$

Miller M, Batt G (1973) Reef and Beach Life of New Zealand, Vol. William Collins (New Zealand) Ltd, Auckland

Nealson KH, Scott J (2006) Ecophysiology of the Genus Shewanella. In: Dworkin M, Falkow S, Rosenberg E, Schleifer KH, Stackebrandt E (eds) The Prokaryotes, Vol 6. Springer-Verlag, New York, NY, p 1133-1151

Neef A (1997) Anwendung der in situ Einzelzell-Identifizierung von Bakterien zur Populationsanalyse in komplexen mikrobiellen Biozönosen. Technische Universität München

Nelson K, Fisher CR (2000) Absence of cospeciation in deep-sea vestimentiferan tube worms and their bacterial endosymbionts Symbiosis 28:1-15

Newman EI, Reddell P (1987) The distribution of mycorrhizas among families of vascular plants. New Phytologist 106:745-751 
Newton LC, McKenzie JD (1995) Echinoderms and oil pollution: A potential stress assay using bacterial symbionts. Marine Pollution Bulletin 31:453-456

Newton LC, McKenzie JD (1998) Development and evaluation of echinoderm pollution assays Echinoderms. Balkema, Rotterdam, San Francisco

Nyholm SV, Stabb EV, Ruby EG, Mcfall-Ngai MJ (2000) Establishment of an animalbacterial association: Recruiting symbiotic vibrios from the environment. Proceedings of the National Academy of Sciences 97:10231-10235

O'Loughlin PM, Waters JM, Roy MS (2002) Description of a new species of Patiriella from New Zealand, and review of Patiriella regularis (Echinodermata, Asteroidea) based on morphological and molecular data. Journal of the Royal Society of New Zealand 32:697-711

Pantos O, Cooney RP, Le Tissier MD, Barer MR, O'Donnell AG, Bythell JC (2003) The bacterial ecology of a plague-like disease affecting the Caribbean coral Montastrea annularis. Environmental Microbiology 5:370-382

Pernthaler A, Pernthaler J, Amann R (2002) Fluorescence In Situ Hybridization and Catalyzed Reporter Deposition for the identification of marine bacteria. Applied and Environmental Microbiology 68:3094-3101

Pettit GR, Herald CL, Doubek DL, Herald DL (1982) Isolation and structure of bryostatin 1. Journal of the American Chemical Society 104:6846-6848

Pfennig N, Trüper HG (1989) Anoxygenic phototrophic bacteria. In: Staley JT, Bryant MP, Pfennig N, Holt JG (eds) Bergey's Manual of Systematic Bacteriology, Vol 3. Williams \& Wilkins, Baltimore, MD, p 1635-1709 
Phillips ML (2006) Interdomain interactions: dissecting animal-bacterial symbioses. BioScience 56:376-381

Pichon D, Gaia V, Norman M, Boucher-Rodoni R (2005) Phylogenetic diversity of epibiotic bacteria in the accessory nidamental glands of squids (Cephalopoda: Loliginidae and Idiosepiidae). Marine Biology 147:1323-1332

Piel J (2004) Metabolites from symbiotic bacteria. Natural Product Reports 21:519-538

Piel J (2006) Bacterial symbionts: prospects for the sustainable production of invertebrate-derived pharmaceuticals. Current Medicinal Chemistry 13:39-50

Preston GM (2004) Plant perceptions of plant growth-promoting Pseudomonas. Philosophical Transactions of the Royal Society of London 359:907-918

Prokic I, Brümmer F, Brigge T, Görtz H, Gerdts G, Schütt C, Elbrächter M, Müller WEG (1998) Bacteria of the genus Roseobacter associated with the toxic dinoflagellate Prorocentrum lima. Protist 149:347-357

Rao RU (2004) Wolbachia in worms: endosymbiont of parasitic filarial nematodes. Recent Research Developments in Experimental Medicine 1:95-113

Rau GH, Hedges JI (1979) Carbon-13 depletion in a hydrothermal vent mussel: suggestion of chemosynthetic food source. Science 203:648-649

Rees GN, Harfoot CG, Janssen PH, Schoenborn L, Kuever J, Lünsdorf H (2002) Thiobaca trueperi gen. nov., sp. nov., a phototrophic purple sulfur bacterium isolated from freshwater lake sediment. International Journal of Systematic and Evolutionary Microbiology 52:671-678

Reiswig HM (1974) Water transport, respiration and energetics of three tropical marine sponges. Journal of Experimental Marine Biology and Ecology 14:231-249 
Reiswig HM (1975) Bacteria as food for temperate-water marine sponges. Canadian Journal of Zoology 53:582-589

Reysenbach AL, Pace NR (1995) Reliable amplification of hyperthermophilic Archaeal 16S rRNA genes by the polymerase chain reaction. In: Robb FT, Place AR (eds) Archaea: A laboratory manual - Thermophiles. Cold Spring Harbour Laboratory Press, New York, N.Y., p 101-107

Riquelme C, Hayashida G, Araya R, Uchida A, Santoni M, Ishida Y (1996) Isolation of a native bacterial strain from the scallop, Argopecten purpuratus, with inhibitory effects against pathogenic vibrios. Journal of Shellfish Research 15:369-374

Ritchie KB (2006) Regulation of microbial populations by coral surface mucus and mucus-associated bacteria. Marine Ecology Progress Series 322:1-14

Roberts D, Billett DSM, McCartney G, Hayes GE (1991) Procaryotes on the tentacles of deep-sea holothurians: A novel from of dietary supplementation. Limnology and Oceanography 36:1447-1452

Rochelle PA (ed) (2001) Environmental Molecular Microbiology: Protocols and Applications, Vol. Horizon Scientific Press, Norwich, U.K.

Rohwer F, Breitbart M, Jara J, Azam F, Knowlton N (2001) Diversity of bacteria associated with the Caribbean coral Montastrea franksi. Coral Reefs 20:85-91

Romero GQ, Benson WW (2004) Leaf domatia mediate mutualism between mites and a tropical tree. Oecologia 140:609-616

Roossinck MJ (2005) Symbiosis versus competition in plant virus evolution. Nature Reviews Microbiology 3:917-924 
Rosenberg E, Koren O, Reshef L, Efrony R, Zilber-Rosenberg I (2007) The role of microorganisms in coral health, disease and evolution. Nature Reviews Microbiology 5:355-362

Ruby EG (1996) Lessons from a cooperative, bacterial-animal association: the Vibrio fischeri-Euprymna scolopes light organ symbiosis. Annual Review of Microbiology 50:591-624

Saffo MB (1992) Invertebrates in endosymbiotic associations. American Zoologist $32: 557-565$

Satomi M, Oikawa H, Yano Y (2003) Shewanella marinintestina sp. nov., Shewanella schlegeliana sp. nov. and Shewanella saire sp. nov., novel eicosapentaenoic-acidproducing marine bacteria isolated from sea animal intestines. International Journal of Systematic and Evolutionary Microbiology 53:491-499

Schaufelberger DE, Koleck MP, Beutler JA, Vatakis AM, Alvarado AB, Andrews P, Marzo LV, Muschik GM, Roach J, Ross JT, Lebherz WB, Reeves MP, Eberwein RM, Rodgers LL, Testerman RP, Snader KM, Forenza S (1991) The large-scale isolation of bryostatin 1 from Bugula neritina following current good manufacturing practices. Journal of Natural Products 54:1265-1270

Schloss PD, Handelsman J (2005) Introducing DOTUR, a computer program for defining operational taxonomic units and estimating species richness. Applied and Environmental Microbiology 71:1501-1506

Schmitt S, Weisz JB, Lindquist N, Hentschel U (2007) Vertical transmission of a phylogenetically complex microbial consortium in the viviparous sponge Ircina felix. Applied and Environmental Microbiology 73:2067-2078 
Scott KM, Cavanaugh CM (2007) CO2 uptake and fixation by endosymbiotic chemoautotrophs from the bivalve Solemya velum. Applied and Environmental Microbiology 73:1174-1179

Silva ES (1990) Intracellular bacteria: the origin of dinoflagellate toxicity. Journal of Environmental Pathology, Toxicology and Oncology 10:124-128

Smith DC (2001) Symbiosis research at the end of the millennium. Hydrobiologia 461:49-54

Sorokin DY, Tourova TP, Kolganova TV, Sjollema KA, Kuenen JG (2002) Thioalkalispira microaerophila gen. nov., sp. nov., a novel lithoautotrophic, sulfur-oxidizing bacterium from a soda lake. International Journal of Systematic and Evolutionary Microbiology 52:2175-2182

Souza Santos H, Sasso WS (1970) Ultrastructural and histochemical studies on the epithelium revestment layer in the tube feet of the starfish Asterina stellifera. Journal of Morphology 130:287-296

Spiridonova EM, Kuznetsov BB, Pimenov NV, Tourova TP (2006) Phylogenetic characterization of endosymbionts of the hydrothermal vent mussel Bathymodiolus azoricus by analysis of the $16 \mathrm{~S}$ rRNA, cbbL, and pmoA genes. Microbiology 75:694-701

Stanley GDJ (2006) Photosymbiosis and the evolution of modern coral reefs. Science 312:857-858

Stanley GDJ, Swart PK (1995) Evolution of the coral-zooxanthellae symbiosis during the Triassic: a geochemical approach. Paleobiology 21:179-199 
Stewart JE, Marks LJ, Wood CR, Risser SM, Gray S (1997) Symbiotic relations between bacteria and the domoic acid producing diatom Pseudonitschia multiseries and the capacity of these bacteria for gluconic acid/gluconolactone formation. Aquatic Microbial Ecology 12:211-221

Strahl ED, Dobson WE, Lundie LLJ (2002) Isolation and screening of brittlestarassociated bacteria for antibacterial activity. Current Microbiology 44:450-459

Suzuki MT, Giovannoni SJ (1996) Bias caused by template annealing in the amplification of mixtures of 16s rRNA genes by PCR. Applied and Environmental Microbiology 62:625-630

Takemura M (2001) Poxviruses and the origin of the eukaryotic nucleus. Journal of Molecular Evolution 52:419-425

Taylor MW, Radax R, Steger D, Wagner M (2007) Sponge-associated microorganisms: evolution, ecology, and biotechnological potential. Microbiology and Molecular Biology Reviews 71:295-347

Temara A, de Ridder C, Kuenen JG, Robertson LA (1993) Sulfide-oxidizing bacteria in the burrowing echinoid, Echinocardium cordatum (Echinodermata). Marine Biology 115:179-185

Theron J, Cloete TE (2000) Molecular techniques for determining microbial diversity and community structure in natural environments. Critical Reviews in Microbiology $26: 37-57$

Thiel V, Leininger S, Schmaljohann R, Brümmer F, Imhoff JF (2007a) Sponge-specific bacterial associations of the Mediterranean sponge Chondrilla nucula (Demospongiae, Tetractinomorpha). Microbial Ecology 54:101-111 
Thiel V, Neulinger SC, Staufenberger T, Schmaljohann R, Imhoff JF (2007b) Spatial distribution of sponge-associated bacteria in the Mediterranean sponge Tethya aurantium. FEMS Microbiology Ecology 59:47-63

Thorsen MS (1999) Abundance and biomass of the gut-living microorganisms (bacteria, protozoa and fungi) in the irregular sea urchin Echinocardium cordatum (Spatangoida: Echinodermata). Marine Biology 133:353-360

Town JC (1980) Diet and food preference of intertidal Astrostole scabra (Asteroidea: Forcipulata). New Zealand Journal of Marine and Freshwater Research 14:427435

Vacelet J, Donadey C (1977) Electron microscope study of the association between some sponges and bacteria. Journal of Experimental Marine Biology and Ecology 30:301-314

Vela AI, Collins MD, Latre MV, Mateos A, Moreno MA, Hutson R, Dominguez L, Fernandez-Garayzabal JF (2003) Psychrobacter pulmonis sp. nov., isolated from the lungs of lambs. International Journal of Systematic and Evolutionary Microbiology 53:415-419

Vetter RD (1985) Elemental sulfur in the gills of three species of clams containing chemoautotrophic symbiotic bacteria: a possible inorganic energy storage compound. Marine Biology 88:33-42

Visick KL, Ruby EG (2006) Vibrio fischeri and its host: it takes two to tango. Current Opinion in Microbiology 9:632-638

Wagner-Döbler I, Biebl H (2006) Environmental biology of the marine Roseobacter lineage. Annual Review of Microbiology 60:255-280 
Walker CW, Lesser MP (1989) Nutrition and development of brooded embryos in the brittlestar Amphipholis squamata: do endosymbiotic bacteria play a role? Marine Biology 103:519-530

Wallner G, Amann R, Beisker W (1993) Optimizing fluorescent in situ hybridization with rRNA-targeted oligonucleotide probes for flow cytometric identification of microorganisms. Cytometry 14:136-143

Wang Y, Brune A, Zimmer M (2007) Bacterial symbionts in the hepatopancreas of isopods: Diversity and environmental transmission. FEMS Microbiology Ecology 61:141-152

Webster NS, Hill RT (2001) The culturable microbial community of the Great Barrier Reef sponge Rhopaloeides odorabile is dominated by an $\alpha$-Proteobacterium. Marine Biology 138:843-851

Wehrl M, Steinert M, Hentschel U (2007) Bacterial uptake by the marine sponge Aplysina aerophoba. Microbial Ecology 53:355-365

Weisz JB, Hentschel U, Lindquist N, Martens CS (2007) Linking abundance and diversity of sponge-associated microbial communities to metabolic differences in host sponges. Marine Biology 152:475-483

Whatmore AM, Dawson CE, Groussaud P, Koylass MS, King AC, Shankster SJ, Sohn AH, Probert WS, McDonald WL (2008) Marine mammal Brucella genotype associated with zoonotic infection. Emerging Infectious Diseases 14:517-518

Wilkinson CR (1978) Microbial associations in sponges. I. Ecology, physiology and microbial populations of coral reef sponges. Marine Biology 49:161-167 
Wilkinson CR (1983) Net primary productivity in coral reef sponges. Science 219:410412

Wilkinson CR (1999) Global and local threats to coral reef functioning and existence: review and predictions. Marine and Freshwater Research 50:867-878

Wilkinson CR, Garrone G, Vacelet J (1984) Marine sponges discriminate between food bacteria and bacterial symbionts: electron microscope radioautography and in situ evidence. Proceedings of the Royal Society of London B 220:519-528

Williamson VM, Gleason CA (2003) Plant-nematode interactions. Current Opinion in Plant Biology 6:327-333

Winn WC, Allen SD, Janda WM, Koneman EW, Procop GW, Schreckenberger PC, Woods GL (eds) (2006a) Koneman's color atlas and textbook of diagnostic microbiology, Vol. Lippincott Williams \& Wilkins, Baltimore, MD

Winn WJ, Allen S, Janda W, Koneman E, Procop G, Schreckenberger P, Woods G (2006b) Koneman's Color Atlas and Textbook of Diagnostic Microbiology, Vol. Lippincott Williams \& Wilkins, Baltimore, MD

Woese CR (1987) Bacterial evolution. Microbiological Reviews 51:221-271

Won Y-J, Jones WJ, Vrijenhoek RC (2008) Absence of cospeciation between deep-sea mytilids and their thiotrophic endosymbionts. Journal of Shellfish Research 27:129-138

Woyke T, Teeling H, Ivanova NN, Huntemann M, Richter M, Gloeckner FO, Boffelli D, Anderson IJ, Barry KW, Shapiro HJ, Szeto E, Kyrpides NC, Mussmann M, Amann R, Bergin C, Ruehland C, Rubin EM, Dubilier N (2006) Symbiosis 
insights through metagenomic analysis of a microbial consortium. Nature 443:950-955

Yu Y, Breitbart M, McNairnie P, Rohwer F (2006) FastGroupII: A web-based bioinformatics platform for analyses of large 16s rDNA libraries. BMC Bioinformatics 7:57

Zobell CE (1941) Studies on marine bacteria. I. The cultural requirements of heterotrophic aerobes. Journal of Marine Research 4:42-75 


\section{Appendix}

\section{Chapter 2 (Phylogenetic analysis of SCB)}

\section{Development of methods}

In order to effectively isolate and identify $\mathrm{SCB}$, it was necessary to first develop appropriate methods. The four key areas in which methods were trialed and modified were the physical sampling of the echinoderms, DNA extraction, the choice of PCR primers, and cloning of PCR products. The alterations made in each of these areas, and the reasons for doing so, are discussed briefly below.

Echinoderm sampling. Sampling methods remained largely unchanged throughout the course of the experiments carried out in this study. However, in order to collect as many bacteria as possible from the three species of shallow-water echinoderms, different methods for sampling wound sites after tissue excision were trialed. Early sampling attempts included the use of sterile cotton gauze and Hybond-N DNA transfer membrane (GE Healthcare) to collect bacteria which may have colonized the wound sites. Briefly, this consisted of trimming a piece of gauze or Hybond-N to the appropriate size and placing it in the wound produced by tissue excision, then leaving it in place for 1 hour. This method did result in successful isolation of DNA, however leaving the echinoderms out of water for 1 hour was not ideal, and this method was discarded in favour of simply swabbing the wound site with a sterile cotton-tipped swab after leaving the echinoderms in $1 \mu \mathrm{m}$-filtered seawater for 1 hour. This method was equally successful at isolating 
bacteria, and was considered preferable despite the risk of seawater-borne bacteria contaminating the wound site.

DNA extraction. Prior to settling on the use of Roche High Pure PCR Template Preparation kit for DNA extraction, two phenol / chloroform based extraction protocols were tried. These protocols are described in detail below.

\section{Protocol 1*}

Tissue samples, surface swabs and seawater filter discs were placed into $15 \mathrm{ml}$ polypropylene screw-top tubes with $1 \mathrm{ml} \mathrm{HTE} \mathrm{buffer}(50 \mathrm{mM}$ Tris- $\mathrm{HCl}, \mathrm{pH}$ 7.5, $20 \mathrm{mM}$ EDTA), $18 \mu \mathrm{l}$ of $18 \mathrm{mg} \mathrm{ml}^{-1}$ proteinase $\mathrm{K}$ and $2 \mu \mathrm{l} 10 \% \mathrm{w} / \mathrm{v}$ sodium dodecyl sulphate (SDS). The mixture was vortexed and incubated for $30 \mathrm{~min}$ at $37{ }^{\circ} \mathrm{C}$. After incubation, $200 \mu \mathrm{l} 2 \% \mathrm{w} / \mathrm{v}$ SDS was added, the mixture was vortexed again, and was incubated for 15 min at $37{ }^{\circ} \mathrm{C} .175 \mu \mathrm{l}$ of $5 \mathrm{M} \mathrm{NaCl}$ was then added and vortexed, and the mixture was put through three cycles of freeze $\left(10\right.$ min at $\left.-80{ }^{\circ} \mathrm{C}\right)$ and thaw $\left(5\right.$ min at $\left.65{ }^{\circ} \mathrm{C}\right)$. Following the final thaw cycle, $170 \mu \mathrm{l}$ of $5 \%$ cetyl trimethyl ammonium bromide (CTAB) in $0.7 \mathrm{M} \mathrm{NaCl}$ was added, vortexed for $1 \mathrm{~min}$, and incubated for $30 \mathrm{~min}$ at $65{ }^{\circ} \mathrm{C} .865 \mu \mathrm{l}$ phenol / chloroform / isoamyl alcohol (25 / 24 / 1) was added to tubes, which were then mixed, and centrifuged for $5 \mathrm{~min}$ at $15,000 \times \mathrm{g} .750 \mu \mathrm{l}$ of the resulting supernatant was transferred from each tube to a $1.5 \mathrm{ml}$ microcentrifuge tube, combined with $750 \mu \mathrm{l}$ chloroform / isoamyl alcohol (24 / 1), vortexed and centrifuged for $5 \mathrm{~min}$ at 15,000 x $\mathrm{g}$. Following centrifugation, $700 \mu \mathrm{l}$ of the supernatant was transferred to a fresh microcentrifuge tube, $420 \mu \mathrm{l}$ isopropanol was added and mixed, and the mixture was 
incubated overnight at $-20{ }^{\circ} \mathrm{C}$. The following day, the mixture was centrifuged for $15 \mathrm{~min}$ at $15,000 \times \mathrm{x}$. The resulting supernatant was discarded, $500 \mu \mathrm{l}$ ice-cold $70 \%$ ethanol was added, and the mixture was centrifuged for $5 \mathrm{~min}$ at $15,000 \mathrm{x}$. This step was then repeated, supernatant was carefully removed, and the DNA pellet was dried in a $37{ }^{\circ} \mathrm{C}$ heating block. Once dry, DNA was resuspended in $100 \mu \mathrm{l}$ sterile double-distilled water and stored at $4{ }^{\circ} \mathrm{C}$ until use. *Protocol modified from (Rochelle 2001).

\section{Protocol 2}

Tissue samples, surface swabs and seawater filter discs were placed into $1.5 \mathrm{ml}$ microcentrifuge tubes with $400 \mu$ sterile TE buffer (10 mM Tris, $1 \mathrm{mM}$ EDTA, pH 8.0). Lysozyme was added to a final concentration of $2 \mathrm{mg} \mathrm{ml}^{-1}$, and the solution was incubated at $37{ }^{\circ} \mathrm{C}$ for $30 \mathrm{~min}$. Sodium Dodecyl Sulfate (SDS) and Proteinase K were then added to final concentrations of $1 \% \mathrm{w} / \mathrm{v}$ and $0.2 \mathrm{mg} \mathrm{ml}^{-1}$ respectively, and the suspension was incubated at $65^{\circ} \mathrm{C}$ for $15 \mathrm{~min}$. Following incubation, $400 \mu \mathrm{l}$ TE-buffered phenol was added and mixed, the tube was centrifuged at $15,000 \times \mathrm{x}$ for $10 \mathrm{~min}$, and the upper aqueous layer was transferred to a fresh microcentrifuge tube. $400 \mu \mathrm{l}$ chloroform was added to the new tube and mixed, the tube was centrifuged at $15,000 \times \mathrm{g}$ for $10 \mathrm{~min}$ and the upper aqueous layer was transferred to a fresh microcentrifuge tube. $1 \mathrm{ml}$ cold 99\% ethanol and $50 \mu 13 \mathrm{M}$ Sodium Acetate were added to the new tube, which was then centrifuged at $15,000 \times \mathrm{g}$ for $20 \mathrm{~min}$. Supernatant was removed, $400 \mu \mathrm{l}$ cold $70 \%$ ethanol was added, and the tube was centrifuged again at 15,000 x $g$ for $5 \mathrm{~min}$. The supernatant was removed and the pellet was dried in a $37{ }^{\circ} \mathrm{C}$ heating block. After drying, the DNA pellet was resuspended in $100 \mu \mathrm{l}$ sterile double-distilled water and stored at $4{ }^{\circ} \mathrm{C}$. 
Unfortunately, both protocols failed to consistently produce a usable DNA pellet, and were therefore discarded in favour of the Roche DNA extraction kit. Protocol 2 was successfully used to extract DNA from cultivated bacterial colonies (See Chapter 4), so the failure to extract DNA from the samples used here is presumably due to the properties of the samples rather than general ineffectiveness of the extraction protocols.

PCR primers. The results reported in this study were obtained using the proteobacteriaspecific PCR primer set PLK1 / ABR1 / GDR2. Prior to developing this set, however, several primer pairs of varying specificity were used in an attempt to amplify DNA from as diverse a range of potential SCB as possible. These primers, and the reason for their disuse, are discussed below (primer sequences, target sites and references can be found in Table A1. In all cases, PCR was carried out on DNA extracted from Patiriella sp., $S$. mollis.

The first primers used in this study were the universal primers 519f (Suzuki \& Giovannoni 1996) and 1406r (Reysenbach \& Pace 1995). It was hoped that, through the use of universal primers, as many bacteria as possible would be targeted. However, the 519f / 1406r primer pair preferentially amplified the DNA of the host echinoderms. Due to the abundance of host DNA relative to bacterial DNA in tissue samples, this resulted in only echinoderm DNA being amplified (when BLAST searching sequences, all near matches belonged to echinoderms closely related to the host species from which samples came). 
The primer pair 27f / 1492r (Lane, 1991), which targets eubacteria, was used next. Initial results using these primers were promising, and provided preliminary evidence that SCB belong to the proteobacteria. However, PCR contamination soon became an issue, with negative control reactions consistently producing amplified DNA. Attempts were made to remedy this issue. These included steam sterilizing all micropipettes and consumables used in PCR setup, UV sterilization of PCR workspaces, micropipettes, consumables and reagents, and the purchase of entirely new PCR reagents. Additionally, PCR conditions were altered in an attempt to reduce the effect of any contaminating DNA. This consisted of incrementally raising the annealing temperature and reducing the number of PCR cycles. Despite these efforts, contamination persisted, and it was therefore decided to use more specific primers, in the hope that these would not target the bacteria that were causing the contamination.

The first of the more specific primers to be used were the $\alpha$-proteobacteria specific 15.5.6f1 and 15.5.6r2. These primers were designed from a multiple sequence alignment, based on the preliminary results obtained through use of the $27 \mathrm{f} / 1492 \mathrm{r}$ primer pair, which suggested that SCB belong to the proteobacteria. Unfortunately, the primers 15.5.6f1 and 15.5.6r2 failed to amplify any DNA, despite altering cycling conditions in an attempt to find the optimum annealing temperature. The failure to amplify anything is assumed to have occurred because the particular bacteria targeted by these primers are not the same as those associated with the echinoderms studied here. 
As well as the $\alpha$-proteobacteria specific primers described above, the archaea-specific primer pair A751f / UA1406r (Baker et al., 2003) were used, to ensure that archaea were not in fact present but not picked up by the eubacterial primers $27 \mathrm{f}$ and 1492r. Despite repeated attempts, DNA was never amplified from samples using this primer pair, and it was therefore assumed that archaea were not present in the echinoderms examined here.

Finally, the primer set PLK1 / ABR1 / GDR2 was developed. This set consists of a previously published eubacteria-specific forward primer (PLK1) (Klaschik et al. 2002), used in conjunction with the newly-designed reverse primers ABR1 (targeting $\alpha$ - and $\beta$ proteobacteria) and GDR2 (targeting $\gamma$ - and $\delta$-proteobacteria). The forward primer PLK1 was chosen due to the wide range of bacteria that it targets. In comparison to other commonly used eubacterial primers, such as 27f, PLK1 targets a very large number of bacteria (this was determined using the Probematch function of the Ribosomal Database Project (Maidak et al. 2001)). The reverse primers ABR1 and GDR2 were designed from a multiple sequence alignment in the phylogenetic software package Vector NTI Advance 10 (Invitrogen), and match a wide range of bacteria within their respective target taxa. 
Table A1. PCR primers trialed in this study, and the reasons for discontinuation of their use.

\begin{tabular}{|c|c|c|c|}
\hline $\begin{array}{l}\text { Primer pair } \\
\text { and source }\end{array}$ & Sequence & $\begin{array}{c}\text { Target } \\
\text { organisms }\end{array}$ & $\begin{array}{l}\text { Reason for } \\
\text { disuse }\end{array}$ \\
\hline $\begin{array}{l}27 f / 1492 \mathrm{r} \\
\text { (Lane 1991) }\end{array}$ & $\begin{array}{l}\text { AGAGTTGATCMTGGCTCAG } \\
\text { / GGTTACCTTGTTACCACTT }\end{array}$ & Eubacteria & $\begin{array}{c}\text { PCR } \\
\text { contamination* }\end{array}$ \\
\hline $\begin{array}{l}\text { 519f / 1406r } \\
\text { (Suzuki \& } \\
\text { Giovannoni } \\
\text { 1996) / } \\
\text { (Reysenbach } \\
\text { \& Pace 1995) }\end{array}$ & $\begin{array}{l}\text { CAGCMGCCGCGGTAATWC } \\
\text { / ACGGGCGGTGTGTRC }\end{array}$ & Universal 16S & $\begin{array}{c}\text { Primers } \\
\text { preferentially } \\
\text { amplified DNA } \\
\text { of echinoderm } \\
\text { host }\end{array}$ \\
\hline $\begin{array}{c}\text { A751f / } \\
\text { UA1406r } \\
\text { (Baker et al. } \\
\text { 2003) }\end{array}$ & $\begin{array}{c}\text { CCGACGGTGAGRGRYGAA / } \\
\text { ACGGGCGGTGWGTRCAA }\end{array}$ & Archaea & $\begin{array}{l}\text { Failed to } \\
\text { amplify }\end{array}$ \\
\hline $\begin{array}{l}\text { 15.5.6f1 / } \\
\text { 15.5.6r2 (This } \\
\text { study) }\end{array}$ & $\begin{array}{c}\text { GGGTGAGTAACACGTGGGAA } \\
/ \\
\text { CCAACTCCCATGGTGTGACG }\end{array}$ & $\begin{array}{c}\alpha- \\
\text { proteobacteria }\end{array}$ & $\begin{array}{l}\text { Failed to } \\
\text { amplify }\end{array}$ \\
\hline
\end{tabular}

* PCR negative controls consistently produced amplified DNA. Ultra-violet sterilization of PCR reagents and workspaces, and purchase of new reagents and primers did not remove contamination. Likewise, alteration of PCR conditions (increasing annealing temperature and reducing cycle number) did not reduce the incidence of contamination.

Cloning. A significant obstacle to obtaining sequence data was the inability to consistently clone PCR products successfully. Prior to the successful use of the TA Cloning Kit (Invitrogen), the PCR Cloning Kit (with pCAPS cloning vector) from Roche Applied Science was used. When using the Roche PCR Cloning Kit, cloning of PCR products was almost always unsuccessful, despite using the same competent $E$. coli cells and growth conditions as were later used successfully with the TA Cloning Kit. Colonies of transformed bacteria did grow, but when colony screens were performed, the bacteria were found not to contain the PCR product insert. The problem likely occurred during ligation of PCR products into the cloning vector, as repeated attempts using different competent DH5a cells (Invitrogen Subcloning Efficiency and Invitrogen MAX 
Efficiency) and slightly altered heat-shock transformation protocols did not alleviate the problem. There is no apparent reason why the Roche PCR Cloning Kit should be any less successful than the TA Cloning Kit. The two kits do use quite different cloning vectors; the Roche kit uses the pCAPS vector, which requires removal of 3' overhangs from PCR products prior to ligation, whereas the Invitrogen kit uses the pCR2.1 vector, in which the 3' overhang of PCR products is used to form a 'sticky end' ligation. However, this does not explain the difference in cloning success between the two kits, as both are commonly used kits from reputable companies. While no answer can be offered as to the reason, after eventually changing to the TA Cloning Kit, cloning was immediately much more successful, and transformed cells containing PCR products were produced fairly consistently.

\section{Phylogenetic trees of bacteria identified from seawater and echinoderm surfaces}

Figures A1-A10 represent 16S rRNA-based phylogenies of echinoderm-associated and seawater-borne bacteria. In all trees, the term "wound" refers to sequences obtained from initial tissue samples, "1hr wound" refers to tissue samples taken 1 hour after initial sampling, and "wound swab" refers to swabs of the wound sites 1 hour after initial sampling. 


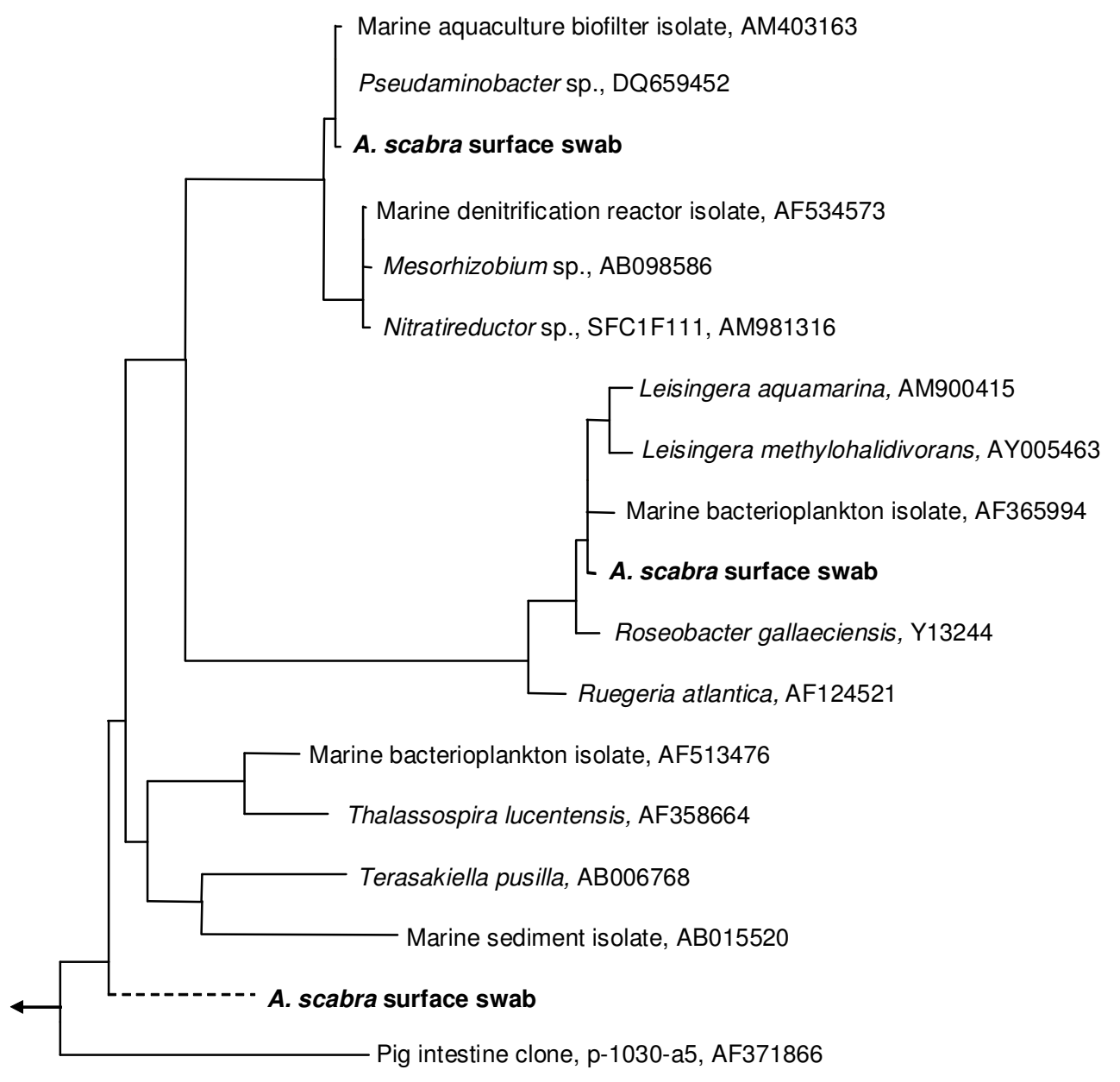

0.10

Figure A1. 16S rRNA-based phylogeny of A. scabra surface-associated $\alpha$-proteobacteria (shown in bold). The displayed tree is a maximum likelihood tree constructed using long (>1,000 nucleotides) sequences. Shorter sequences (indicated by dashed lines) were added using the parsimony interactive tool in ARB. 
Appendix

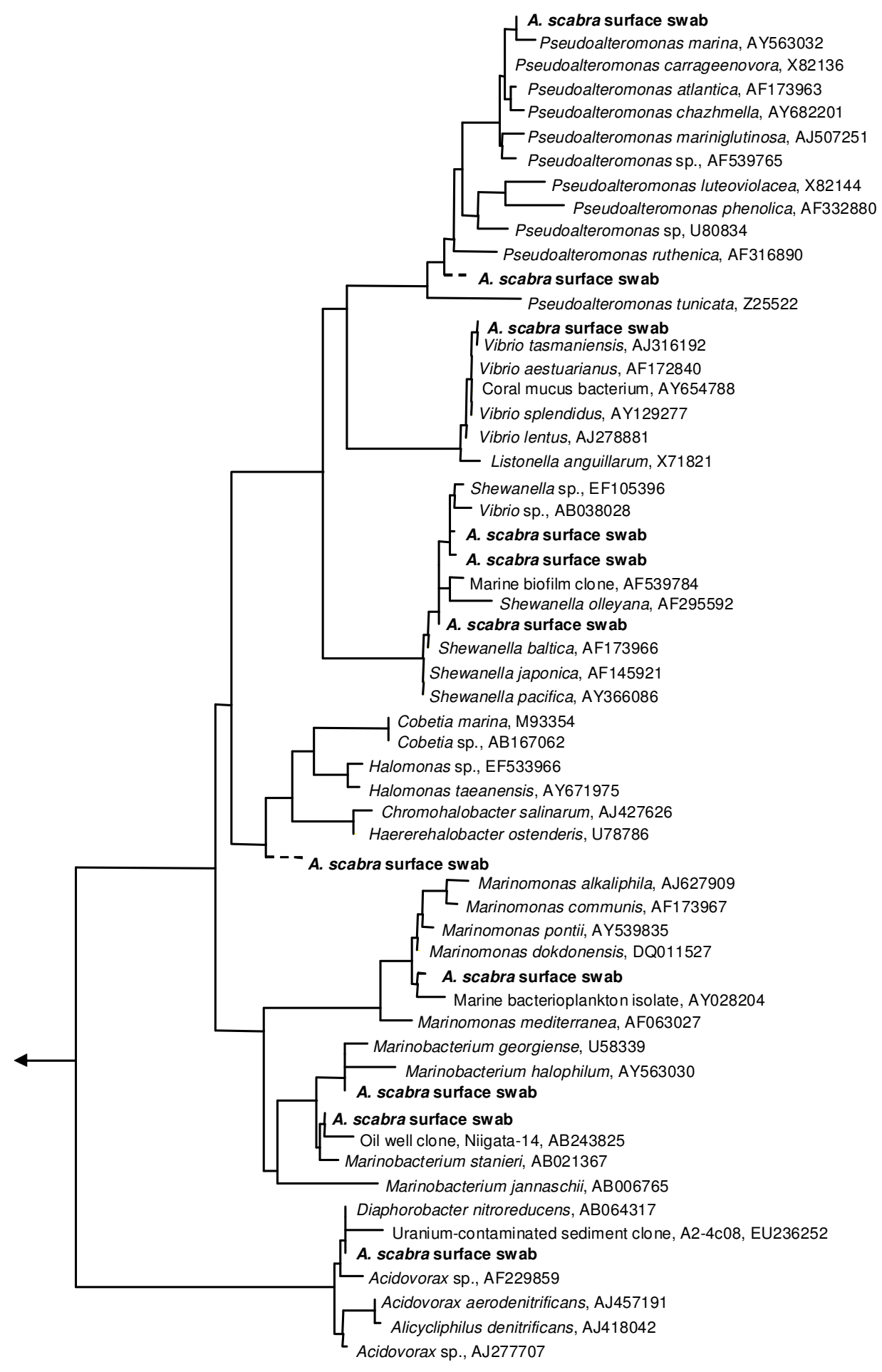

0.10

Figure A2. 16S rRNA-based phylogeny of A. scabra surface-associated $\gamma$ - and $\beta$-proteobacteria (shown in bold). The displayed tree is a maximum likelihood tree constructed using long $(>1,000$ nucleotides) sequences. Shorter sequences (indicated by dashed lines) were added using the parsimony interactive tool in ARB. 


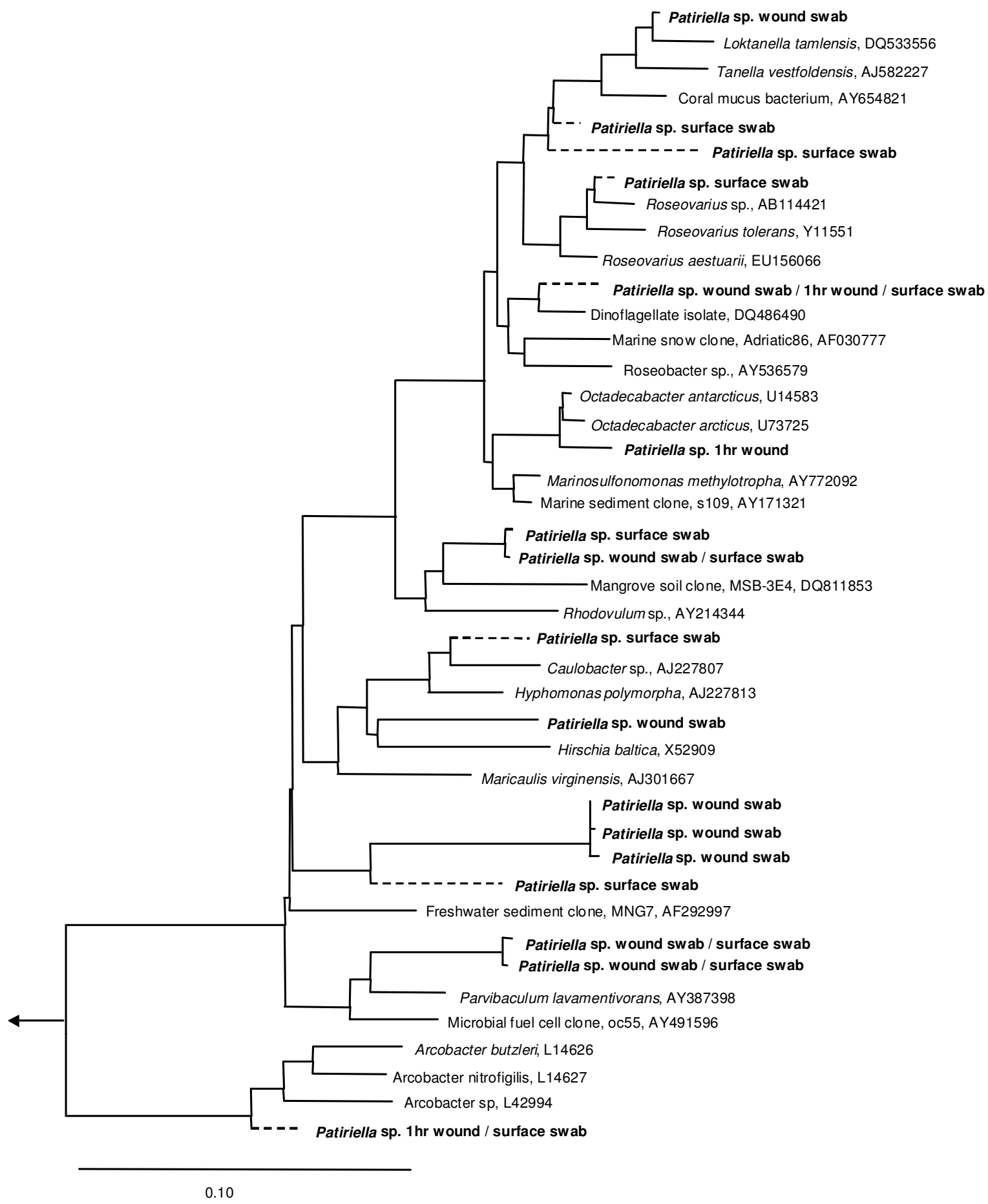

Figure A3. 16S rRNA-based phylogeny of Patiriella sp. -associated $\alpha$ - and $\varepsilon$-proteobacteria (shown in bold). The displayed tree is a maximum likelihood tree constructed using long ( $>1,000$ nucleotides) sequences. Shorter sequences (indicated by dashed lines) were added using the parsimony interactive tool in ARB. 
Appendix

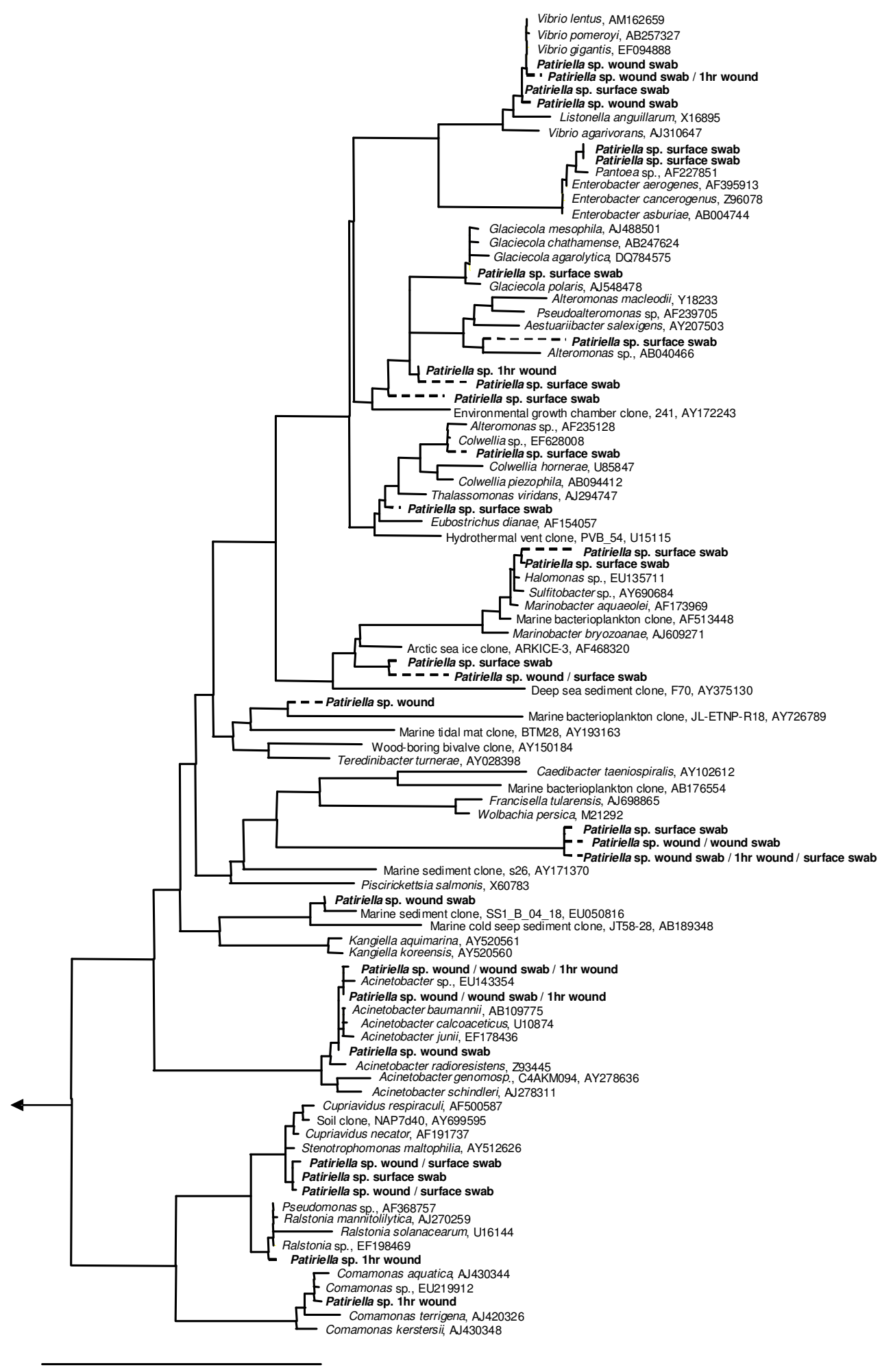

0.10

Figure A4. 16S rRNA-based phylogeny of Patiriella sp.-associated $\gamma$ - and $\beta$-proteobacteria (shown in bold). The displayed tree is a maximum likelihood tree constructed using long ( $>1,000$ nucleotides) sequences. Shorter sequences (indicated by dashed lines) were added using the parsimony interactive tool in ARB. 
Appendix

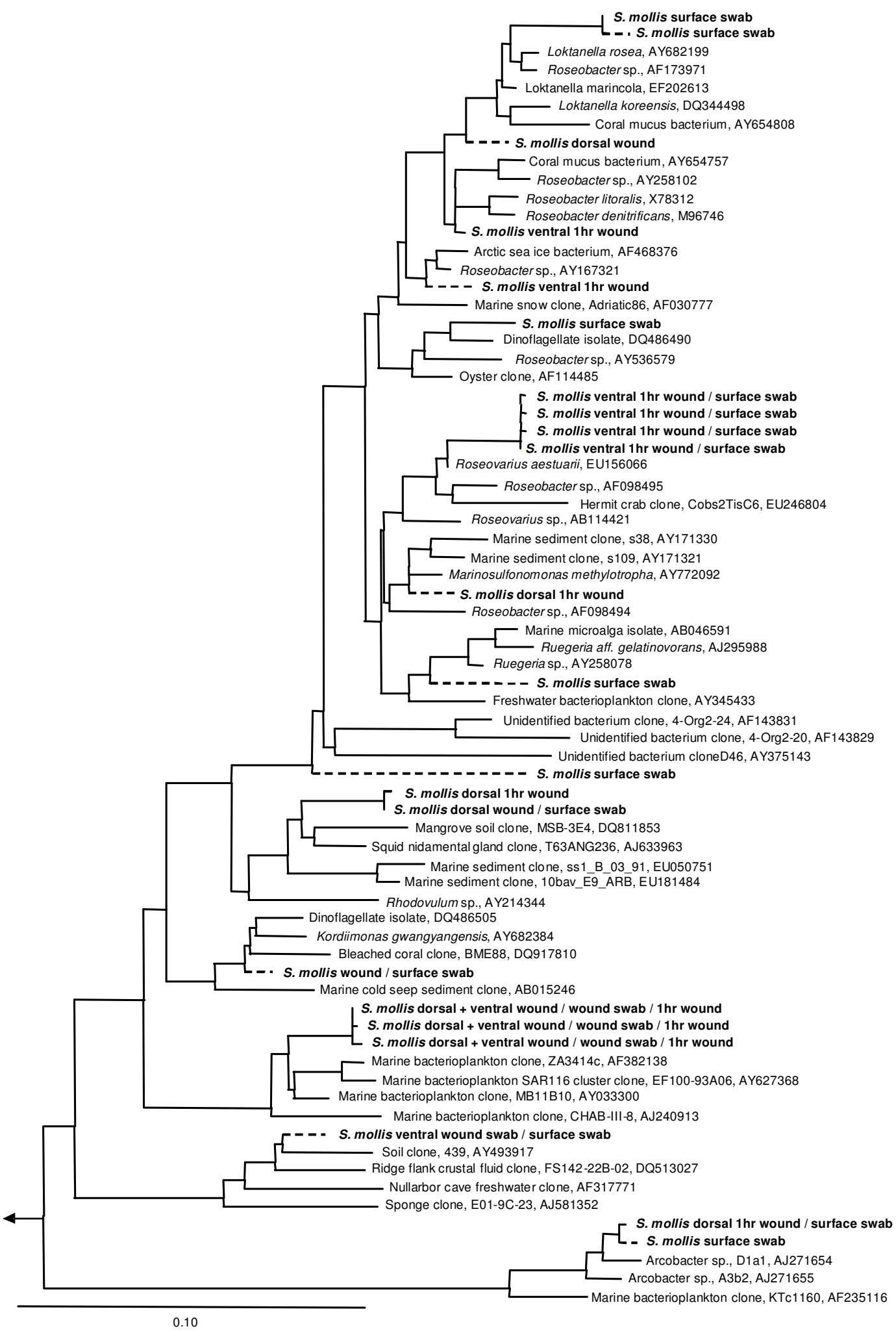

Figure A5. 16S rRNA-based phylogeny of S. mollis.-associated $\alpha$ - and $\varepsilon$-proteobacteria (shown in bold). The displayed tree is a maximum likelihood tree constructed using long $(>1,000$ nucleotides) sequences. Shorter sequences (indicated by dashed lines) were added using the parsimony interactive tool in ARB. 
Appendix

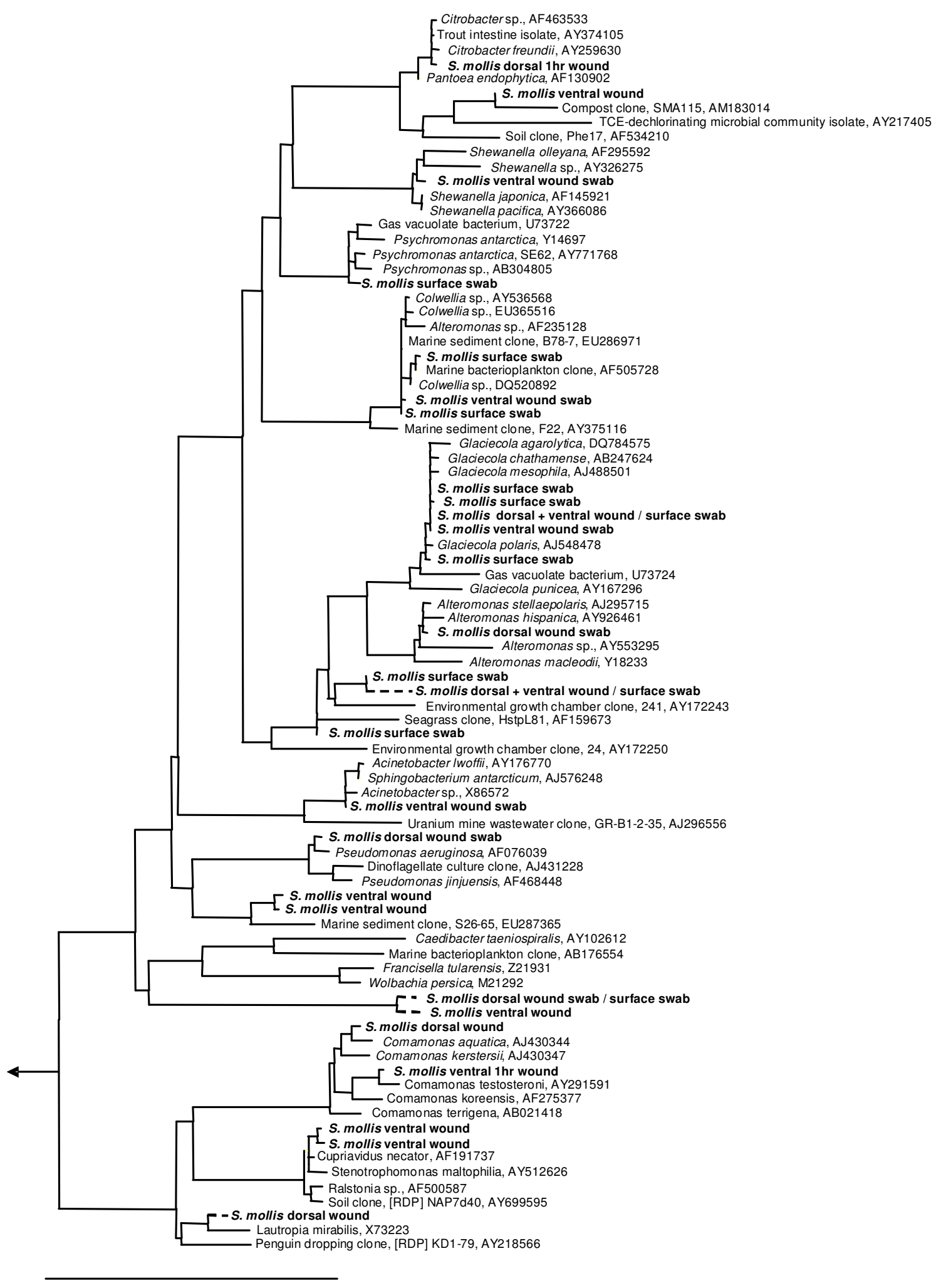

0.10

Figure A6. $16 \mathrm{~S}$ rRNA-based phylogeny of S. mollis.-associated $\gamma$ - and $\beta$-proteobacteria (shown in bold). The displayed tree is a maximum likelihood tree constructed using long $(>1,000$ nucleotides) sequences. Shorter sequences (indicated by dashed lines) were added using the parsimony interactive tool in ARB. 
Appendix

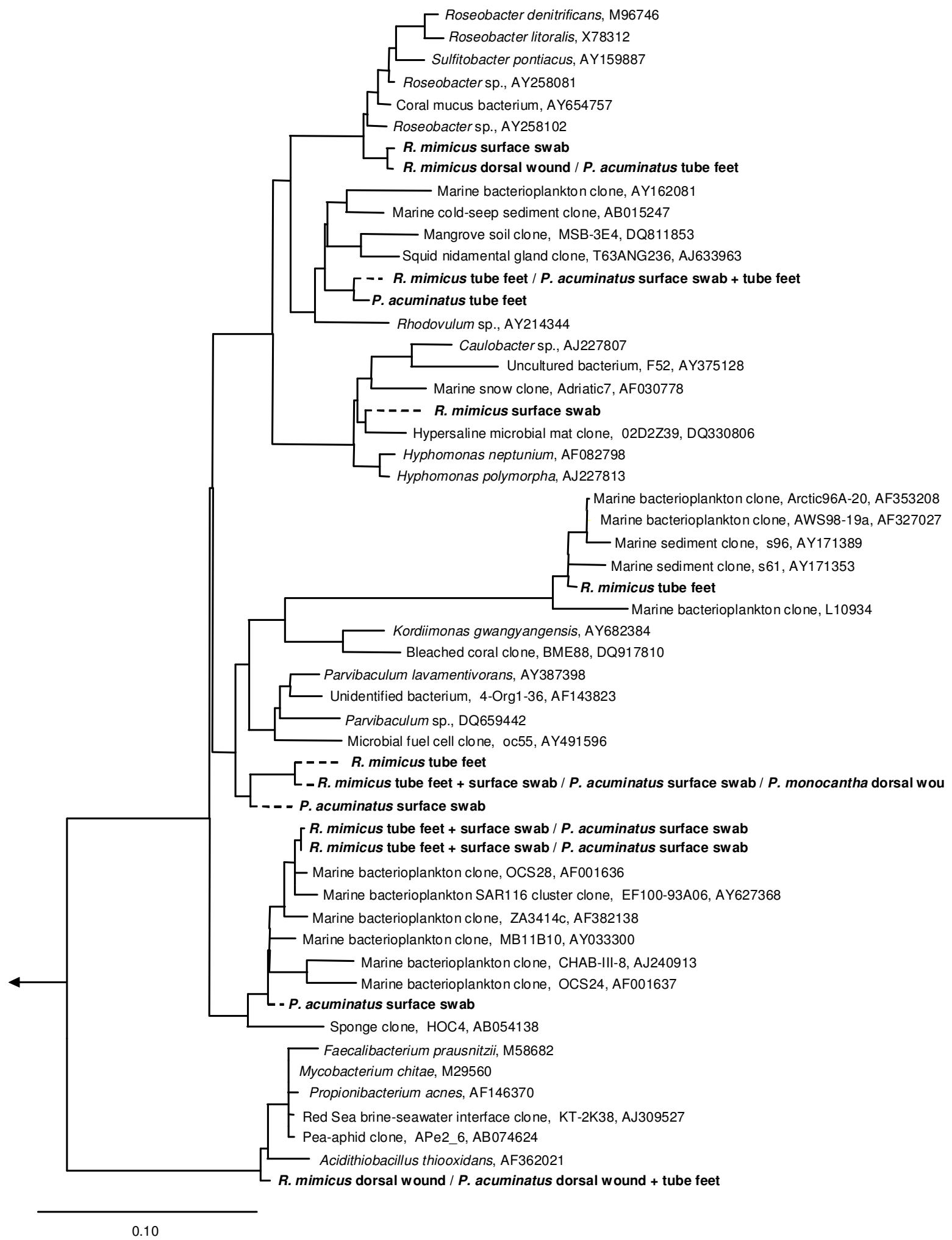

Figure A7. 16S rRNA-based phylogeny of Perissasterias monocantha-, Psilaster acuminatus- and Rosaster mimicus-associated $\alpha$-proteobacteria (shown in bold). The displayed tree is a maximum likelihood tree constructed using long ( $>1,000$ nucleotides) sequences. Shorter sequences (indicated by dashed lines) were added using the parsimony interactive tool in ARB. 
Appendix

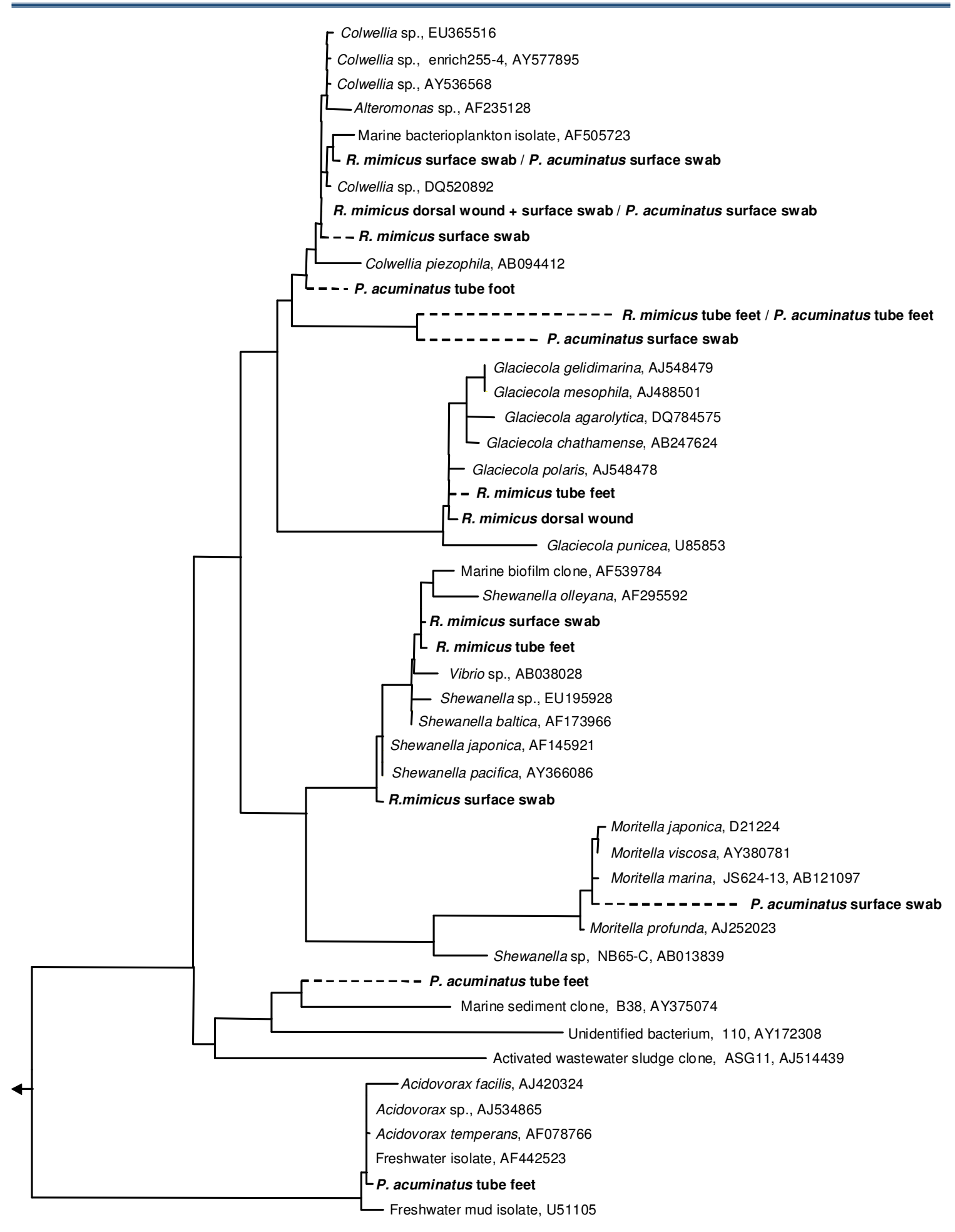

0.10

Figure A8. 16S rRNA-based phylogeny of Psilaster acuminatus- and Rosaster mimicus-associated $\gamma$ - and $\beta$-proteobacteria (shown in bold). The displayed tree is a maximum likelihood tree constructed using long ( $>1,000$ nucleotides) sequences. Shorter sequences (indicated by dashed lines) were added using the parsimony interactive tool in ARB. 
Appendix

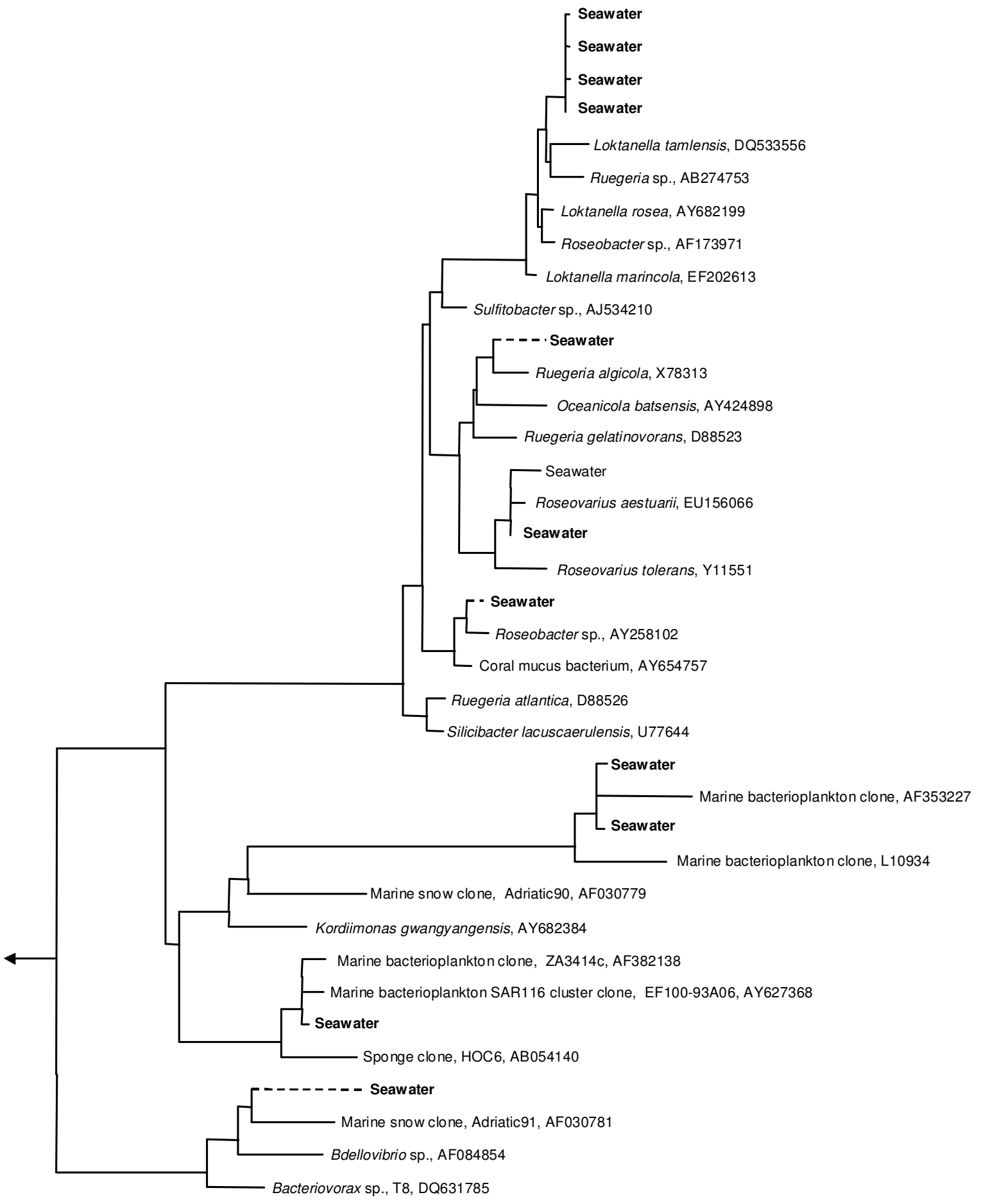

0.10

Figure A9. 16S rRNA-based phylogeny of $\alpha$ - and $\delta$-proteobacteria isolated from seawater (shown in bold). The displayed tree is a maximum likelihood tree constructed using long ( $>1,000$ nucleotides) sequences. Shorter sequences (indicated by dashed lines) were added using the parsimony interactive tool in ARB. 
Appendix

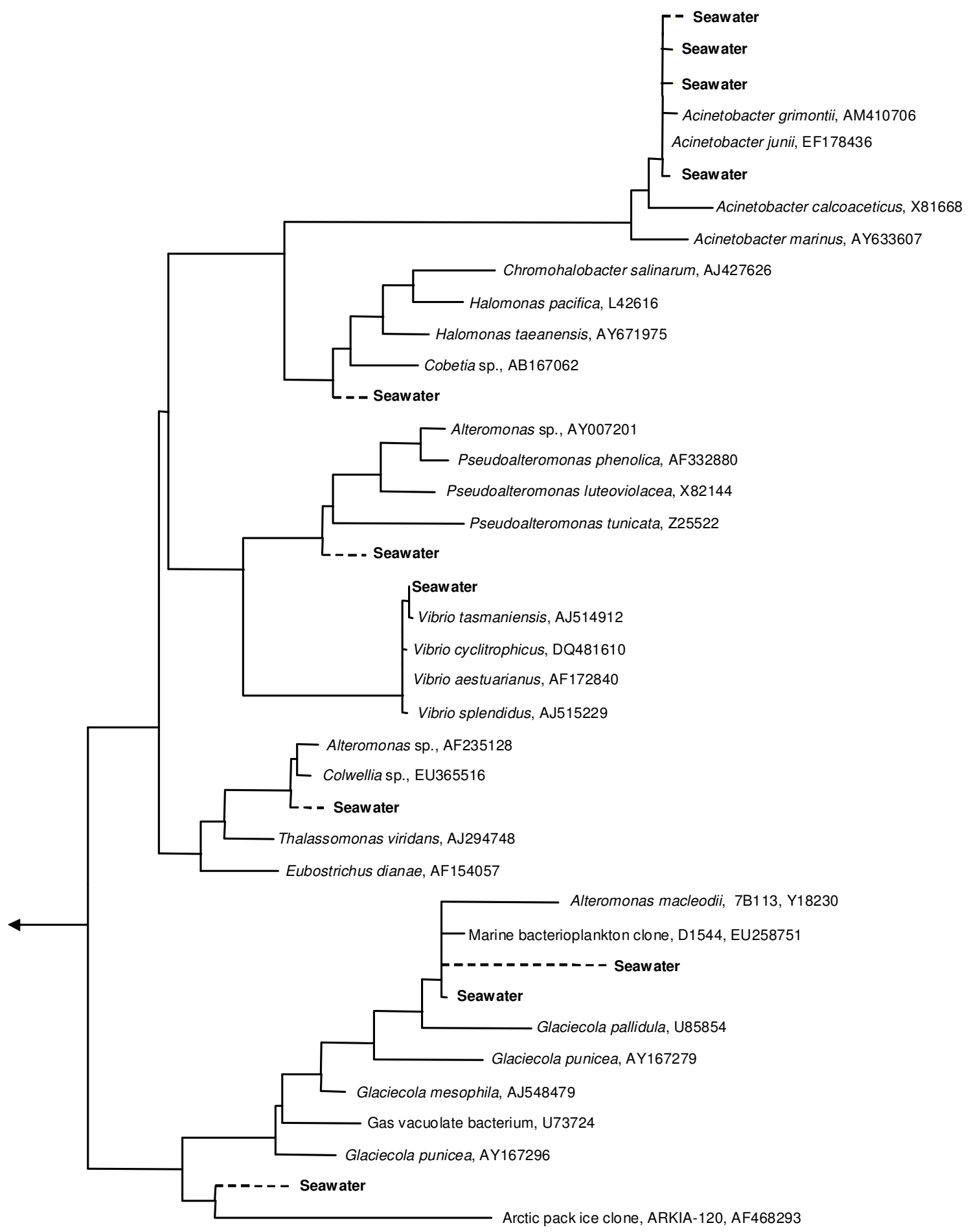

0.10

Figure A10. 16S rRNA-based phylogeny of $\gamma$-proteobacteria isolated from seawater (shown in bold). The displayed tree is a maximum likelihood tree constructed using long ( $>1,000$ nucleotides) sequences. Shorter sequences (indicated by dashed lines) were added using the parsimony interactive tool in ARB. 


\section{Chapter 3 (FISH analysis of SCB)}

\section{Standard FISH assays}

Table A2. Formula for FISH hybridization buffer

\begin{tabular}{cc}
\hline Constituent (concentration) & Amount \\
\hline Sodium chloride (5 M) & $270 \mu \mathrm{l}$ \\
Tris/HCl (1 M) & $30 \mu \mathrm{l}$ \\
Sodium dodecyl sulphate (SDS) (10\%) & $1.5 \mu \mathrm{l}$ \\
Formamide (100\%) & Depends on desired concentration: $0 \mu \mathrm{l}(0 \%), 300 \mu \mathrm{l}(20 \%)$, \\
Double-distilled water & $525 \mu \mathrm{l}(35 \%), 750 \mu \mathrm{l}(50 \%)$ \\
& $\begin{array}{c}\text { Depends on desired formamide concentration: } 1198 \mu \mathrm{l} \\
(0 \%), 898 \mu \mathrm{l}(20 \%), 674 \mu \mathrm{l}(35 \%), 448 \mu \mathrm{l}(50 \%)\end{array}$ \\
\hline
\end{tabular}

Buffer is prepared in a $1.5 \mathrm{ml}$ microcentrifuge tube. SDS is added last to prevent precipitation.

Table A3. Formula for FISH wash buffer

Constituent (concentration)

Amount

Tris/HCl (1 M)

$\operatorname{EDTA}(0.5 \mathrm{M})$

SDS $(10 \%)$

Sodium chloride (5 M)

Double-distilled water
$1 \mathrm{ml}$

$500 \mu \mathrm{l}$ (only when using formamide concentrations of $20 \%$ or greater)

$50 \mu 1$

Depends on formamide concentration used in hybridizations: $9 \mathrm{ml}(0 \%), 2.15 \mathrm{ml}(20 \%), 700 \mu \mathrm{l}$ (35\%), $180 \mu \mathrm{l}(50 \%)$

To a total volume of $50 \mathrm{ml}$

Buffer is prepared in a $50 \mathrm{ml}$ polypropylene screw-top tube. SDS is added last to prevent precipitation. 
Table A4. Specificity of oligonucleotide probes SCB1 and SCB2

\begin{tabular}{|c|c|c|c|c|c|c|c|c|c|c|c|c|c|c|c|c|c|c|c|c|}
\hline \multirow[b]{2}{*}{$<\mathrm{T}^{\circ} \mathrm{C}$} & \multicolumn{20}{|c|}{ Number of non-target matches with decreasing temperature } \\
\hline & 0.3 & 0.6 & 0.9 & 1.2 & 1.5 & 1.8 & 2.1 & 2.4 & 2.7 & 3.0 & 3.3 & 3.6 & 3.9 & 4.2 & 4.5 & 4.8 & 5.1 & 5.4 & 5.7 & 6.0 \\
\hline \multicolumn{21}{|l|}{ Probe } \\
\hline SCB1 & 0 & 0 & 0 & 0 & 0 & 0 & 0 & 0 & 0 & 0 & 3 & 3 & 3 & 27 & 33 & 33 & 35 & 61 & 74 & 87 \\
\hline SCB2 & 0 & 0 & 0 & 0 & 0 & 0 & 0 & 0 & 0 & 0 & 0 & 0 & 12 & 17 & 17 & 29 & 35 & 40 & 65 & 75 \\
\hline \multicolumn{21}{|c|}{$\begin{array}{l}\text { Specificity of oligonucleotide probes } \mathrm{SCB} 1 \text { and SCB2 as temperature decreases away from optimal } \\
\text { hybridization temperature }\left(46.5^{\circ} \mathrm{C} \text { for SCB } 1,45.6^{\circ} \mathrm{C} \text { for SCB2). Both probes are robust to changes in }\right. \\
\text { hybridization stringency, with a hybridization temperature decrease of at least } 3^{\circ} \mathrm{C} \text { not affecting specificity. } \\
\text { Even at lower temperatures than this, non-specific binding is not considered an issue, as none of the non- } \\
\text { target matches are bacteria that were identified in any samples through DNA sequencing (See Chapter } 2 \text { ). }\end{array}$} \\
\hline
\end{tabular}

\section{CARD-FISH assays}

Table A5. Formula for CARD-FISH hybridization buffer

Constituent (concentration) Amount

$\begin{array}{cc}\text { Sodium chloride }(5 \mathrm{M}) & 360 \mu \mathrm{l} \\ \text { Tris-HCl (1 M) } & 40 \mu \mathrm{l} \\ \text { Dextran sulphate } & 0.2 \mathrm{~g} \\ \text { Formamide (100\%) } & 0.4-1.4 \mathrm{ml}(20-70 \%) \\ \text { Double-distilled water } & 0-1 \mathrm{ml} \text { (Depending on formamide concentration) } \\ \text { Blocking reagent (10\%) } & 200 \mu \mathrm{l} \\ \text { SDS (10\%) } & 2 \mu \mathrm{l}\end{array}$

Mixture is incubated at $48-60{ }^{\circ} \mathrm{C}$ until dextran sulphate dissolves. 
Table A6. Formula for CARD-FISH wash buffer

\begin{tabular}{cc}
\hline Constituent (concentration) & Amount \\
\hline Sodium chloride (5 M) & $0-8.9 \mathrm{ml}$ (depending on formamide concentration) \\
Tris-HCl (1 M) & $1 \mathrm{ml}$ \\
EDTA $(0.5 \mathrm{M})$ & $500 \mu \mathrm{l}$ \\
SDS (10\%) & $50 \mu \mathrm{l}$ \\
Double-distilled water & To a total volume of $50 \mathrm{ml}$ \\
\hline
\end{tabular}

SDS is added last to prevent precipitation.

Table A7. Formula for CARD-FISH amplification buffer

Constituent (concentration) $\quad$ Amount

PBS $(20 x)$

$2 \mathrm{ml}$

Blocking reagent (10\%)

$0.4 \mathrm{ml}$

Sodium chloride (5 M)

$16 \mathrm{ml}$

Dextran sulphate

$4 \mathrm{~g}$

Double-distilled water

To a final volume of $40 \mathrm{ml}$

Buffer is prepared in a $50 \mathrm{ml}$ tube and is heated to $48-60{ }^{\circ} \mathrm{C}$ to dissolve dextran sulphate. 

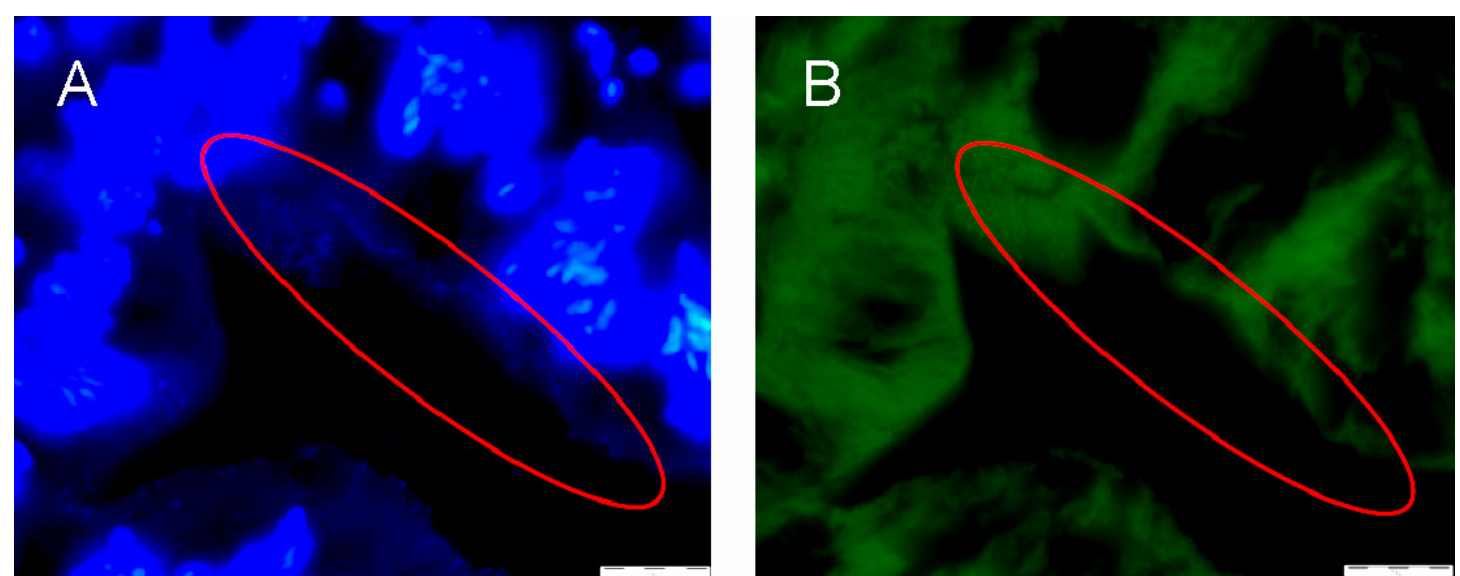

Figure A11. Result of CARD-FISH assay using the FITC-labeled eubacterial probe EUB338 on Patiriella sp. tissue sections. A: DAPI-stained section. Note what appear to be SCB (circled), faintly visible near the edge of the tissue. B: The same section, showing FITC fluorescence. The same area is circled, but SCB are not visible due to autofluorescence of the echinoderm tissue. Scale bars represent $20 \mu \mathrm{m}$.
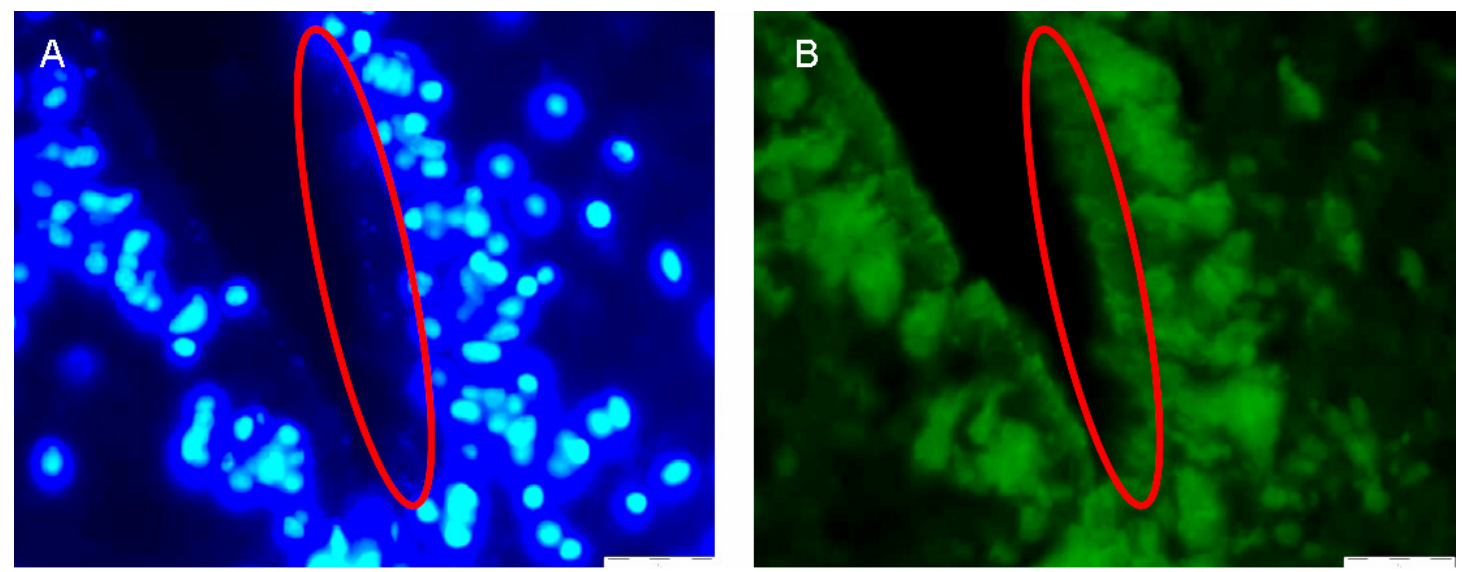

Figure A12. Result of CARD-FISH assay using the FITC-labeled eubacterial probe EUB338 on S. mollis tissue sections. A: DAPI-stained section. Note what appear to be SCB (circled), faintly visible near the edge of the tissue. B: The same section, showing FITC fluorescence. The same area is circled, but SCB are not visible due to autofluorescence of the echinoderm tissue. Scale bars represent $20 \mu \mathrm{m}$. 


\section{Chapter 4 (Cultivation of SCB)}

Table A8. Approximate formula of Difco Marine Broth 2216.

\begin{tabular}{ll}
\hline Salt & Amount per Litre \\
\hline Peptone & $5.0 \mathrm{~g}$ \\
Yeast Extract & $1.0 \mathrm{~g}$ \\
Ferric Citrate & $0.1 \mathrm{~g}$ \\
Sodium Chloride & $19.45 \mathrm{~g}$ \\
Magnesium Chloride & $8.8 \mathrm{~g}$ \\
Sodium Sulfate & $3.24 \mathrm{~g}$ \\
Calcium Chloride & $1.8 \mathrm{~g}$ \\
Potassium Chloride & $0.55 \mathrm{~g}$ \\
Sodium Bicarbonate & $0.16 \mathrm{~g}$ \\
Potassium Bromide & $0.08 \mathrm{~g}$ \\
Strontium Chloride & $34.0 \mathrm{mg}$ \\
Boric Acid & $22.0 \mathrm{mg}$ \\
Sodium Silicate & $4.0 \mathrm{mg}$ \\
Sodium Fluoride & $2.4 \mathrm{mg}$ \\
Ammonium Nitrate & $1.6 \mathrm{mg}$ \\
Disodium Phosphate & $8.0 \mathrm{mg}$ \\
Agar & $15.0 \mathrm{~g}$ \\
\hline & \\
\hline
\end{tabular}


Table A9. Formula of Zobell modified 2216E medium.

\begin{tabular}{lc}
\hline Salt & Amount per Litre \\
\hline Peptone & $1.0 \mathrm{~g}$ \\
Yeast Extract & $1.0 \mathrm{~g}$ \\
Artificial Seawater & To 1 Litre \\
\hline
\end{tabular}

Table A10. Formula of Artificial Seawater used in this study.

\begin{tabular}{lc}
\hline Salt & Amount per Litre \\
\hline Sodium Chloride & $24.6 \mathrm{~g}$ \\
Potassium Chloride & $0.67 \mathrm{~g}$ \\
Calcium Chloride. $2 \mathrm{H}_{2} \mathrm{O}$ & $1.36 \mathrm{~g}$ \\
Magnesium Sulfate. $7 \mathrm{H}_{2} \mathrm{O}$ & $6.29 \mathrm{~g}$ \\
Magnesium Chloride. $6 \mathrm{H}_{2} \mathrm{O}$ & $4.66 \mathrm{~g}$ \\
Sodium Bicarbonate & $0.18 \mathrm{~g}$ \\
\hline
\end{tabular}

